\title{
Effective-field theories for heavy quarkonium
}

\author{
Nora Brambilla \\ INFN and Dipartimento di Fisica dell'Università di Milano via Celoria 16, 20133 Milan, Italy \\ Antonio Pineda and Joan Soto \\ Departament d'Estructura i Constituents de la Matèria, Universitat de Barcelona, Diagonal \\ 647, E-08028 Barcelona, Catalonia, Spain
}

Antonio Vairo

INFN and Dipartimento di Fisica dell'Università di Milano via Celoria 16, 20133 Milan, Italy

(Published 2 December 2005)

\begin{abstract}
This article reviews recent theoretical developments in heavy-quarkonium physics from the point of view of effective-field theories of QCD. We discuss nonrelativistic QCD and concentrate on potential nonrelativistic QCD. The main goal will be to derive Schrödinger equations based on QCD that govern heavy-quarkonium physics in the weak- and strong-coupling regimes. Finally, the review discusses a selected set of applications, which include spectroscopy, inclusive decays, and electromagnetic threshold production.
\end{abstract}

\section{CONTENTS}

I. Introduction

II. NRQCD
A. Degrees of freedom
B. Power counting
C. Lagrangian, currents, and symmetries
D. Matching
E. Renormalization group
F. Applications: spectrum and inclusive decay widths
1. Spectra
2. Inclusive decay widths

III. Potential NRQCD. The Physical Picture
A. Weak-coupling regime

B. Strong-coupling regime

IV. Potential NRQCD. The Weak-Coupling Regime
A. pNRQCD: the degrees of freedom
B. Power counting
C. Lagrangian and symmetries
D. Feynman rules
E. Matching: diagrammatic approach
1. Pinch singularities
2. Potentials

F. Matching: Wilson-loop approach

1. Interpolating fields

2. Matching at $\mathcal{O}\left(r^{0}, 1 / m^{0}\right)$

3. Matching at $\mathcal{O}\left(r^{1}, 1 / m^{0}\right)$ and $\mathcal{O}\left(r^{2}, 1 / m^{0}\right)$

4. Matching at order $r^{0}\left(1 / m, 1 / m^{2}\right.$, and beyond)

G. Observables: spectrum and inclusive decay widths

1. Heavy-quarkonium mass

2. Inclusive decay widths

H. Renormalization group

V. Renormalons and the Definition of the Heavy-Quark Mass

A. The pole mass and static singlet potential renormalon
1450

1450

1452

1453

1454

1456

1456
B. Renormalon-subtracted scheme and power counting VI. (p)NRQCD: The Static Limit

A. NRQCD in the static limit

B. Static pNRQCD in the weak-coupling regime

C. The singlet static potential at short distances versus lattice

D. Gluelumps versus lattice

VII. Potential NRQCD. The Strong-Coupling Regime
A. Degrees of freedom

B. Power counting

C. Lagrangian and symmetries

D. Matching: analytic and nonanalytic mass terms

E. Matching for $|\mathbf{p}| \sim \Lambda_{\mathrm{QCD}}$

1. Matching of the analytic terms: quantum-mechanical matching

2. Matching of the analytic terms: the real pNRQCD potential

3. Matching of the analytic terms: the imaginary pNRQCD potential

4. Matching of the analytic terms: direct matching of Wilson-loop amplitudes

5. Matching of the nonanalytic terms

F. Matching for $|\mathbf{p}| \gg \Lambda_{\mathrm{OCD}} \gg E$

1. pNRQCD $^{\prime}$

2. Matching pNRQCD to $\mathrm{pNRQCD}$

G. Potentials and spectra: lattice and models

1. Potentials and spectrum from the lattice

2. QCD vacuum models

H. Inclusive decay widths into light particles

1458

1459

1459

1460

1461

1462

1463

1463

1464

1464

1464

1465

1466

1467

1470

1471

1472

1474

1474

1474

1477

1478

1479

1480

1482

1482

1483

B. Spectroscopy in the weak-coupling regime

C. Electromagnetic inclusive decay widths in the weak-coupling regime

D. Inclusive decay widths in the strong-coupling regime 1485

E. Nonrelativistic sum rules

1487

F. $t-\bar{t}$ production near threshold

1488 


\author{
G. Semi-inclusive radiative decays \\ IX. Conclusions \\ X. Prospects \\ Acknowledgments \\ Table of Acronyms \\ References
}

1489

1491

1492

1493

1493

1493

\section{INTRODUCTION}

In order to understand human-scale processes, a classical nonrelativistic (NR) picture of physics based on Galilean symmetry proves sufficient. Until the beginning of the last century, this picture, supplemented with electromagnetism, was enough to understand the majority of processes observed in nature. At the start of the quantum age, it was again a NR equation, the Schrödinger equation, which proved to be the most successful in explaining the atomic and nuclear spectra.

High-energy processes are far from human-scale processes. They are described in the present by relativistic quantum field theories (QFTs). Under some circumstances, however, high-energy processes develop a NR regime and produce bound states that behave very much like atoms.

The discovery of the $J / \psi$, a heavy resonance with a very narrow width, at Brookhaven and SLAC (Aubert et al., 1974; Augustin et al., 1974), which was later identified with a bound state of a new (heavy) quark, charm, and its antiquark, namely, a charmonium $(c \bar{c})$ state, opened up the possibility of using a NR picture in the realm of QCD, the fundamental QFT of strong interactions. This possibility was enhanced three years later by the discovery of the $Y$, an even heavier and narrower resonance, which was again interpreted as a bound state of a new (heavier) quark, bottom, and its antiquark, namely, a bottomonium $(b \bar{b})$ state (Herb et al., 1977). In fact, the narrow width of these resonances proved to be crucial in establishing QCD as the sector of the Standard Model that describes the strong interaction (Appelquist and Politzer, 1974; De Rujula and Glashow, 1975). From that moment on, charmonia and bottomonia have been throughly studied and still are a subject of intensive theoretical and experimental research (see, for instance, Brambilla et al., 2004 and Skwarnicki, 2004). They can indeed be classified in terms of the quantum numbers of a NR bound state, and the spacing of the radial excitations and of the fine and hyperfine splittings has a pattern similar to the ones in positronium, a well-studied QED NR bound state. A related system, the $b \bar{c}$ bound state $\left(B_{c}\right)$, has also been found in nature (Abe et al., 1998). The heaviest of the quarks, the top, which has recently been found at the Tevatron (Abe et al., 1994), has a large decay width (due to weak interactions) and is not expected to form narrow $t-\bar{t}$ resonances. However, the production of $t-\bar{t}$ near threshold, namely, in the NR regime, will be one of the major programs at the International Linear Collider.

These systems will be denoted by heavy quarkonia. They are characterized by at least three widely sepa- rated scales: the hard scale (the mass $m$ of the heavy quarks), the soft scale (the relative momentum of the heavy quark-antiquark $|\mathbf{p}| \equiv p \sim m v, v \ll 1$ ), and the ultrasoft (US) scale (the typical kinetic energy $E \sim m v^{2}$ of the heavy quark and antiquark). Moreover, by the definition of heavy quark, $m$ is large in comparison with the typical hadronic scale $\Lambda_{\mathrm{QCD}}$. Hence processes that happen at the scale $m$ are expected to be successfully described using perturbation theory, due to the asymptotic freedom of QCD. This explains why the narrow heavyquarkonium widths could be qualitatively understood as a manifestation of asymptotic freedom. However, lower scales, such as $|\mathbf{p}|$ and $E$, which are responsible for the binding, may or may not be accessible to perturbation theory. The appearance of all these scales in the dynamics of heavy quarkonia makes its quantitative study extremely difficult. This is even so in the weak-coupling regime, where the system becomes Coulombic. Nevertheless, by exploiting the hierarchies $m \gg p \gg E$ and $m$ $\gg \Lambda_{\mathrm{QCD}}$ the problem can be considerably simplified. This may be done in any particular calculation for a given observable, or, alternatively, using effective-field theories (EFTs). In the latter, the hierarchies of scales are exploited at the action level producing universal results independent of particular observables, which is far more advantageous. The basic idea behind EFTs is that to describe observables of a particular (low) energy region, one can integrate out the degrees of freedom of the other regions. This produces an effective action (for the EFT) involving only the degrees of freedom in the region we are interested in. Calculating with the effective (EFT) or with the fundamental (QCD) action gives equivalent physical results as far as that particular region is concerned, but calculations are much simpler with the EFT. In heavy quarkonium, we are interested in physics at the low-energy scale $E$. Hence EFTs, which have energy scales larger than $E$ integrated out, can be and have been built. They have led to major progress in our understanding of heavy quarkonium in recent years. We shall devote this review to these new developments. Before that, let us put this progress in a historical perspective.

The discovery of bottomonium and charmonium triggered the use of NR potential models (where the physics of the bound state is described by a Schrödinger equation). The main input in this approach is the potential introduced. At lowest order in the weak-coupling regime $\left(|\mathbf{p}| \gg \Lambda_{\mathrm{QCD}}\right)$, the potential is Coulombic. Higherorder corrections to the potential in perturbation theory were obtained over the years (Buchmüller et al., 1981; Gupta and Radford, 1981, 1982; Pantaleone et al., 1986; Titard and Yndurain, 1994) even though the computations were difficult due to the several scales involved. It was also not clear how to systematically incorporate US effects. For instance, we mention the infrared sensitivity found in the static potential (Appelquist et al., 1978) or in the one-loop calculations of $P$-wave decays (Barbieri et al., 1980). In any case, the observed bottomonium and charmonium spectra turned out not to be Coulombic 
and phenomenologically fine-tuned potentials were necessary to reproduce them (Eichten et al., 1978). See Brambilla and Vario (1999a) for more references. This motivated attempts to derive the heavy-quarkonium potential from QCD without relying on perturbation theory. The idea was to find gauge-invariant expressions for the potentials (within an expansion in $1 / \mathrm{m}$ ) in terms of the expectation values of Wilson loops. Several methods have been worked out over the years and expressions for the spin-dependent and spin-independent potentials up to $\mathcal{O}\left(1 / \mathrm{m}^{2}\right)$ were obtained (Wilson, 1974; Susskind, 1977; Brown and Weisberger, 1979; Eichten and Feinberg, 1981; Peskin, 1983; Gromes, 1984; Barchielli et al., 1988, 1990; Szczepaniak and Swanson, 1997). All the obtained potentials have been investigated on the lattice (see Bali, 2001, for a recent review). However, these results had a number of shortcomings. Lucha et al. (1991) pointed out that if calculated in perturbation theory, the potentials obtained from the Wilson-loop approach missed the hard logarithms $\sim \ln m$ present in the potentials directly computed from QCD. More recently, Brambilla, Pineda, et al. (2001) also pointed out that not only hard logarithms, but some of the potentials, relevant at relative order $\alpha_{s}^{2}$ in the spectrum, were missed as well. Finally, the infrared (IR) divergences in the perturbative computation of $P$-wave decays seemed impossible to accommodate in that framework. Overall, a more systematic and controlled derivation of the nonrelativistic dynamics from QCD was required.

Independent of the line of research above, nonrelativistic QED (NRQED) an EFT for NR leptons, was introduced (Caswell and Lepage, 1986). It turned out to be the first and decisive link in the chain of developments that we shall review here. NRQED is obtained from QED by integrating out the hard scale $m$. It is characterized by an UV cutoff much smaller than the mass $m$ and much larger than any other scale. Nonrelativistic QCD (NRQCD), which also appears in the title of the article of Caswell and Lepage (1986), was introduced soon afterwards (Lepage and Thacker, 1988). The Lagrangian of NRQCD can be organized in powers of $1 / m$, thus making explicit its nonrelativistic nature. A set of operators and matching coefficients are associated with each power of $m$. The operators encode the lowenergy content of the theory and the coefficients encode the effects of the degrees of freedom with energy $\mathcal{O}(m)$ that have been integrated out. Namely, in NRQCD the contributions coming from the hard scale $m$ are factorized. NRQCD had two major advantages that we would like to point out here: (i) it could be rigorously derived from QCD in a systematic manner providing an optimized framework for lattice simulations (Thacker and Lepage, 1991) and (ii) it solved the problem of the IR divergences of the $P$-wave decays of heavy quarkonium. This solution, however, came at the price of introducing the so-called color-octet matrix elements, which could not be incorporated in the Schrödinger-like formulations available at that time. In spite of this, it was noted by
Chen et al. (1995) that if the nonperturbative potentials were calculated starting from NRQCD instead of from QCD, the problem of the missing hard logarithms mentioned above disappeared. ${ }^{1}$ This again raised some hope that NR potential models could eventually be regarded as EFTs of QCD. It also made it evident that the potential models available, even those in which the potentials were obtained in terms of Wilson loops, were not controlled derivations from QCD and that first-principles computations of heavy quarkonia should be done within the framework of NRQCD.

NRQCD itself was, however, not free of shortcomings. The main problem was related to the fact that both soft and US degrees of freedom were entangled. This affects the power-counting rules, which were not homogeneous. Namely, the power counting by Lepage et al. (1992) assumed $\Lambda_{\mathrm{QCD}} \leq m v^{2}$, which catches the leadingorder contribution but not the subleading contributions in $v$. Also perturbative calculations were difficult to perform due to the dependence on two scales. Another problem was that the first computations in NRQCD were based on cutoff regularization, ${ }^{2}$ whereas the calculations in QCD are often done in dimensional regularization (DR). Attempts to perform the matching between QCD and NRQCD using DR had the drawback that the naive incorporation of the kinetic term in the quark propagator jeopardized the power-counting rules.

A solution to the last problem was first proposed by Manohar (1997). He argued that the matching between QCD and NRQCD in the bilinear sector of the theory in DR should be performed by treating the kinetic-energy term as a perturbation, as was done in heavy-quark effective theory (reviewed by Neubert, 1994). Along the same lines, the matching of QCD to NRQCD in the four-fermion sector, where the Coulomb pole enhancement starts playing a role, was performed soon after by Pineda and Soto (1998a, 1998c). The key point was that in order to carry out the matching, it is not so important to know the power counting of each term in the effective theory, but to know that the remaining dynamical scales of the effective theory are much lower than the mass: $m \gg|\mathbf{p}|, E, \Lambda_{\mathrm{QCD}}$.

Returning to the main problem, the first works addressing the entanglement of the soft and US scales in NRQCD tried to classify the different momentum regions existing in a purely perturbative version of NRQCD and/or to reformulate NRQCD in such a way that some of these regions were explicitly displayed by introducing new fields in the NRQCD Lagrangian. In particular, we mention the article by Labelle (1998),

\footnotetext{
${ }^{1}$ These are included in the matching coefficients of the theory and may be transferred to the potentials by expanding Green's functions in NRQCD instead of in QCD (Chen et al., 1995; Bali et al., 1997; Brambilla and Vairo, 1999b).

${ }^{2}$ In any case, the simplifications compared with purely relativistic Bethe-Salpeter-like (Bethe and Salpeter, 1951) computations were enormous and led to a plethora of new results in QED. See, for instance, Kinoshita and Nio, 1996; Hoang et al., 1997; Labelle et al., 1997; Hill and Lepage, 2000; Hill, 2001.
} 
where a diagrammatic approach to NRQED was used and the subsequent work by Luke and Manohar (1997), Grinstein and Rothstein (1998), and Luke and Savage (1998) in NRQCD. All these early attempts turned out to be missing some relevant intermediate degrees of freedom.

The first complete solution came in the work of Pineda and Soto (1998a). The idea was to build an EFT containing only the degrees of freedom relevant for

$\bar{Q}-Q$ systems near threshold, i.e., those with $E \sim m v^{2}$ and as close as possible to a Schrödinger-like formulation [see also Lepage (1997)]. All other degrees of freedom were to be integrated out. The EFT, which was called potential NRQCD (pNRQCD), had roughly the following structure:

$$
\left.\begin{array}{rl}
\mathcal{L}= & \Phi(\mathbf{r})^{\dagger}\left(i \partial_{0}-\frac{\mathbf{p}^{2}}{2 m}-V^{(0)}(r)\right. \\
& + \text { corrections to the potential } \\
& + \text { interactions with other low- } \\
& \text { energy degrees of freedom }) \Phi(\mathbf{r})
\end{array}\right\} \text { pNRQCD, }
$$

where $V^{(0)}(r)$ is the static potential $\left(-C_{F} \alpha_{S} / r\right.$ in the perturbative case) and $\Phi(\mathbf{r})$ is the field associated with the

$\bar{Q}-Q$ state. This EFT turned out to meet all our expectations: it achieved the factorization between US and higher-energy modes, had a definite power counting (at least in the perturbative regime), and was very close to a NR Schrödinger-like formulation of the heavyquarkonium dynamics. In the Lagrangian, potentials appear. These are the matching coefficients of the theory and are calculated by matching with NRQCD amplitudes, using either Feynman diagrams (see Beneke et al., 1999; Czarnecki et al., 1999b; Pineda and Soto, 1999; Kniehl, Penin, Steinhauser, et al., 2002 for specific examples in QCD and QED and Sec. IV.E for further details) or Wilson-loop amplitudes (Brambilla et al., 1999b, 2000). In the perturbative regime, a confirmation that pNRQCD was able to catch all the relevant dynamical regions came from diagrammatic studies. Beneke and Smirnov (1998) made the most complete classification of (perturbative) momentum regions to date by a diagrammatic study called the threshold expansion. In the language of the threshold expansion, the matching between NRQCD and QCD corresponds to integrating out the hard region and pNRQCD is obtained from NRQCD by integrating out what are called soft quarks and gluons and potential gluons. Finally, we mention two later works which dealt with reformulating NRQCD within an effective Lagrangian formalism. In the article by Griesshammer (1998) all degrees of freedom of NRQCD were made explicit in the Lagrangian. In that of Luke et al. (2002) the question of how to obtain renormalizationgroup (RG) equations for NR systems was addressed for the first time. The resulting formalism is now known as velocity NRQCD. For a review on this theory, see Hoang (2002). All these formulations should be equivalent to $\mathrm{pNRQCD}$ once the same degrees of freedom have been integrated out.

This closed the circle connecting QCD with a properly modified Schrödinger-like formulation in the weakcoupling regime. Compared with the traditional methods, perturbative computations are optimized since only one scale appears in each of the Feynman integrals. The interaction with US gluons is treated in a quantum-fieldtheory fashion yet everything can be encoded in a Schrödinger-like formulation. The applications of these ideas to QED have also been very successful. We refer to Sec. IV.G for references.

The natural question then was: What happens in the strong-coupling regime? The application of EFTs has led to a well-founded connection with QCD in this regime. The potentials are now understood as matching coefficients to be obtained by comparison with NRQCD. This along with new computational techniques has solved the problems mentioned before allowing the complete computation of the potential at $\mathcal{O}\left(1 / \mathrm{m}^{2}\right)$ (Brambilla, Pineda, et al., 2001; Pineda and Vairo, 2001). Also with these techniques, new terms, nonanalytic in $1 / m$, were identified (Brambilla et al., 2004) and a solution was found for the IR sensitivity of the $P$-wave decays (Brambilla, Eiras, et al., 2002). Again, the use of EFTs has allowed us to close the circle and connect QCD with a properly modified Schrödinger-like formulation in the strongcoupling regime.

Heavy quarkonium has been important in the history of QCD and maintains that role even today. In the early 1970s, its high-energy nature helped to establish asymptotic freedom and QCD as the fundamental theory of the strong interaction. Later on, its NR nature served as a testing ground for many models of the lowenergy dynamics of QCD. Since the 1990s, due to the rise of EFTs for heavy quarks, heavy-quarkonium observables can be rigorously derived from QCD, low- and high-energy modes factorized, and large logarithms systematically resummed. From a conceptual point of view, the origin and exact meaning of a QCD Schrödinger equation has been clarified. In the weak-coupling regime, this opens up the possibility of having precise determinations of the Standard Model parameters to which heavy quarkonium is sensitive: $\alpha_{s}$ and the heavyquark masses. In the strong-coupling regime, heavy quarkonia are, due to their wealth of scales, an ideal laboratory in which to probe the structure of the QCD vacuum.

It is our aim to review the recent developments in heavy-quarkonium physics from the point of view of EFTs. Our main goal will be to derive the QCD Schrödinger equation that governs heavy-quarkonium physics in the weak- and strong-coupling regimes. We shall not be exhaustive in most of the derivations but concentrate on the main ideas and general lines of development with some illustrative examples. Then we shall discuss a selected set of applications. The review is not exhaustive. For instance, we shall not discuss one of the major phenomenological successes of NRQCD: explaining the heavy-quarkonium production rate at the 
Tevatron (Braaten et al., 1996; Beneke, 1997; Krämer, 2001; Bodwin et al., 2003).

Before moving to the main body of the review, we list here our main notational choices (Yndurain, 1999). The QCD Lagrangian density reads

$$
\mathcal{L}=\sum_{i=1}^{N_{f}} \bar{q}_{i}\left(i \not D-m_{i}\right) q_{i}-\frac{1}{4} G^{\mu \nu a} G_{\mu \nu}^{a},
$$

where $D_{\mu}=\partial_{\mu}+i g A_{\mu}, i g G_{\mu \nu}=\left[D_{\mu}, D_{\nu}\right], q_{i}$ are the quark fields, and $m_{i}$ their current masses. $N_{f}$ is the total number of quark flavors. We shall often indicate with the capital letter $Q_{i}$ the heavy-quark fields and always set to zero the light-quark masses. In the EFT, the heavyquark masses will be also indicated by $m_{i}$, but always understood, if not differently specified, as pole masses. The strong-coupling constant $\alpha_{s}=g^{2} / 4 \pi$ in the presence of $n_{f}$ light quarks runs, at energies below the heavyquark thresholds, as

$$
\nu \frac{d \alpha_{s}}{d \nu}=-2 \alpha_{s}\left\{\beta_{0} \frac{\alpha_{s}}{4 \pi}+\beta_{1}\left(\frac{\alpha_{s}}{4 \pi}\right)^{2}+\cdots\right\},
$$

where

$$
\begin{aligned}
& \beta_{0}=\frac{11}{3} C_{A}-\frac{4}{3} T_{F} n_{f}, \\
& \beta_{1}=\frac{34}{3} C_{A}^{2}-\frac{20}{3} C_{A} T_{F} n_{f}-4 C_{F} T_{F} n_{f}, \ldots,
\end{aligned}
$$

and $C_{A}=N_{c}=3, C_{F}=\left(N_{c}^{2}-1\right) / 2 N_{c}=4 / 3$ and $T_{F}=1 / 2$.

The basic computational techniques for perturbative QCD used throughout can be found in the book of Pascual and Tarrach (1984).

\section{NRQCD}

\section{A. Degrees of freedom}

NRQCD is designed to describe the dynamics of a heavy quark and a heavy antiquark (not necessarily of the same flavor) at energy scales (in the center-of-mass frame) much smaller than their masses, which are assumed to be much larger than $\Lambda_{\mathrm{QCD}}$, the typical hadronic scale. At these energies, further heavy-quarkantiquark pairs cannot be created so it is sufficient, and convenient, to use Pauli spinors for both the heavyquark and heavy-antiquark degrees of freedom. We shall denote by $\psi(x)$ the Pauli spinor field that annihilates a quark and by $\chi(x)$ the one that creates an antiquark. Both $\psi(x)$ and $\chi(x)$ transform in the fundamental representation of color SU(3). The remaining (light) degrees of freedom are the same as in QCD, except for the UV cutoffs as we shall discuss below. In particular, the gluon fields will appear in covariant derivatives $D_{\mu}$ and field strengths $G_{\mu \nu}$. For instance, we shall see that the leading-order Lagrangian density for the heavy-quark and antiquark fields reads

$$
\mathcal{L}=\psi^{\dagger}\left(i D_{0}+\frac{1}{2 m} \mathbf{D}^{2}\right) \psi+\chi^{\dagger}\left(i D_{0}-\frac{1}{2 m} \mathbf{D}^{2}\right) \chi .
$$

In a NR frame, the energy and three-momentum of the heavy particles scale in a different way and hence a different UV cutoff may be introduced for each: $\nu_{s}$ and $\nu_{p}$, respectively. However, NRQCD is usually considered as having a single UV cutoff $\nu_{\mathrm{NR}}=\left\{\nu_{p}, \nu_{s}\right\}$ satisfying $E, p, \Lambda_{\mathrm{QCD}} \ll \nu_{\mathrm{NR}} \ll m ; \nu_{p}$ is the UV cutoff of the relative three-momentum of the heavy quark and antiquark; $\nu_{s}$ is the UV cutoff of the energy of the heavy quark and the heavy antiquark, and of the four-momentum of the gluons and light quarks.

From a Wilson RG point of view, NRQCD is obtained from QCD by integrating out energy fluctuations about the heavy-quark (heavy-antiquark) mass and threemomentum fluctuations up to the scale $\nu_{\mathrm{NR}}$ for the heavy-quark (heavy-antiquark) fields, and fourmomentum fluctuations up to the same scale for the fields of the light degrees of freedom. Since $\nu_{\mathrm{NR}}$ $\gg \Lambda_{\mathrm{QCD}}$, this can be carried out in practice perturbatively in $\alpha_{s}\left(\nu_{\mathrm{NR}}\right)$. Within the threshold expansion framework (Beneke and Smirnov, 1998), this corresponds to integrating out the hard modes of QCD.

If the quark and antiquark have the same flavor, they can annihilate into hard gluons, which have already been integrated out and are not present in the NRQCD Lagrangian. This implies that the QCD Lagrangian must contain imaginary Wilson coefficients. The nonHermiticity of the NRQCD Lagrangian, which at first sight may appear rather unwelcome, if not disastrous, turns out to provide an extremely powerful tool for calculating inclusive decay widths to light particles.

\section{B. Power counting}

From the discussion above, it follows that the NRQCD Lagrangian can be organized as a power series in $1 / m_{Q}$ (and $\left.1 / m_{Q}\right)$. The Wilson or matching coefficients of each operator depend logarithmically on $m_{Q}\left(m_{Q}^{-}\right), \nu_{\mathrm{NR}}$ and, as mentioned before, can be calculated in perturbation theory in $\alpha_{s}\left(\nu_{\mathrm{NR}}\right)$. Hence the importance of a given operator for a practical calculation depends not only on its size (power counting), which we shall briefly discuss next, but also on the leading power of $\alpha_{s}$ that its matching coefficient has.

Since several scales $\left(E,|\mathbf{p}|, \Lambda_{\mathrm{QCD}}\right)$ remain dynamical, it is not possible to assign a size to each operator unambiguously without extra assumptions: no homogeneous power counting exists. As we shall see, the introduction of pNRQCD facilitates this task. The original power counting introduced by Bodwin et al. (1995) assumes $\Lambda_{\mathrm{QCD}} \sim E \sim m v^{2}$, and hence $|\mathbf{p}| \sim m v \gg \Lambda_{\mathrm{QCD}}, v \sim \alpha_{s}(m v)$ $\ll 1$, which implies that the bound state is Coulombic (positroniumlike). In this case homogeneous powercounting rules can be given using pNRQCD in the weak-coupling regime (Sec. IV). Nevertheless, it is unlikely that the whole heavy-quarkonium spectrum can be described by this power counting and alternatives 
need to be explored. We only anticipate here that in the strong-coupling regime of pNRQCD the following scaling will be considered: $E \ll|\mathbf{p}| \sim \Lambda_{\mathrm{OCD}}$. The issue of the power counting of NRQCD has also been addressed by Beneke (1997) and Fleming et al. (2001) (see also the discussion in Sec. II.F). In both cases, the authors allow for some freedom in the possible size of the NRQCD matrix elements by introducing a parameter $\lambda$ that interpolates between different power countings.

\section{Lagrangian, currents, and symmetries}

The allowed operators in the Lagrangian are constrained by the symmetries of QCD. However, due to the particular kinematic region on which we are focusing, Lorentz invariance is not linearly realized in the heavy-quark sector, and it is not straightforward (though certainly possible, as will be discussed below) to implement. One has, in a first stage, to content oneself with implementing the rotational subgroup only. Including $n_{f}$ light quarks, the NRQCD Lagrangian density for a quark of mass $m_{1}$ and an antiquark of mass $m_{2}\left(m_{1}, m_{2}\right.$ $\gg \Lambda_{\mathrm{QCD}}$ ) reads at $\mathcal{O}\left(1 / m^{2}\right),{ }^{3} m=m_{1}, m_{2}$ (Caswell and Lepage, 1986; Bodwin et al., 1995; Manohar, 1997; Bauer and Manohar, 1998):

$$
\begin{aligned}
& \mathcal{L}_{\mathrm{NRQCD}}=\mathcal{L}_{g}+\mathcal{L}_{l}+\mathcal{L}_{\psi}+\mathcal{L}_{\chi}+\mathcal{L}_{\psi \chi} \\
& \mathcal{L}_{g}=-\frac{1}{4} G^{\mu \nu a} G_{\mu \nu}^{a}+\frac{1}{4}\left(\frac{c_{1}^{g(1)}}{m_{1}^{2}}+\frac{c_{1}^{g(2)}}{m_{2}^{2}}\right) g f_{a b c} G_{\mu \nu}^{a} G_{\alpha}^{\mu b} G^{\nu \alpha c} \\
& \mathcal{L}_{l}=\sum_{i=1}^{n_{f}} \bar{q}_{i} l I D q_{i}+\frac{1}{8}\left(\frac{c_{1}^{l l(1)}}{m_{1}^{2}}+\frac{c_{1}^{l l(2)}}{m_{2}^{2}}\right) g^{2} \sum_{i, j=1}^{n_{f}} \bar{q}_{i} T^{a} \gamma^{\mu} q_{i} \bar{q}_{j} T^{a} \gamma_{\mu} q_{j} \\
&+\frac{1}{8}\left(\frac{c_{2}^{l l(1)}}{m_{1}^{2}}+\frac{c_{2}^{l l(2)}}{m_{2}^{2}}\right) g^{2} \sum_{i, j=1}^{n_{f}} \bar{q}_{i} T^{a} \gamma^{\mu} \gamma_{5} q_{i} \bar{q}_{j} T^{a} \gamma_{\mu} \gamma_{5} q_{j} \\
&+\frac{1}{8}\left(\frac{c_{3}^{l l(1)}}{m_{1}^{2}}+\frac{c_{3}^{l l(2)}}{m_{2}^{2}}\right) g^{2} \sum_{i, j=1}^{n_{f}} \bar{q}_{i} \gamma^{\mu} q_{i} \bar{q}_{j} \gamma_{\mu} q_{j} \\
&+\frac{1}{8}\left(\frac{c_{4}^{l l(1)}}{m_{1}^{2}}+\frac{c_{4}^{l l(2)}}{m_{2}^{2}}\right) g^{2} \sum_{i, j=1}^{n_{f}} \bar{q}_{i} \gamma^{\mu} \gamma_{5} q_{i} \bar{q}_{j} \gamma_{\mu} \gamma_{5} q_{j}, \\
& \mathcal{L}_{\psi}= \psi^{\dagger}\left\{i D_{0}+\frac{c_{k}^{(1)}}{2 m_{1}} \mathbf{D}^{2}+\frac{c_{4}^{(1)}}{8 m_{1}^{3}} \mathbf{D}^{4}+\frac{c_{F}^{(1)}}{2 m_{1}} \boldsymbol{\sigma} \cdot g \mathbf{B}\right. \\
&+\frac{c_{D}^{(1)}}{8 m_{1}^{2}}(\mathbf{D} \cdot g \mathbf{E}-g \mathbf{E} \cdot \mathbf{D})+i \frac{c_{S}^{(1)}}{8 m_{1}^{2}} \boldsymbol{\sigma} \cdot(\mathbf{D} \times g \mathbf{E} \\
&-g \mathbf{E} \times \mathbf{D})\} \psi+\frac{c_{1}^{h l(1)}}{8 m_{1}^{2}} g^{2} \sum_{i=1}^{n_{f}} \psi^{\dagger} T^{a} \psi \bar{q}_{i} \gamma_{0} T^{a} q_{i} \\
&+\frac{c_{2}^{h l(1)}}{8 m_{1}^{2}} g^{2} \sum_{i=1}^{n_{f}} \psi^{\dagger} \sigma^{k} T^{a} \psi \bar{q}_{i} \gamma_{k} \gamma_{5} T^{a} q_{i} \\
&
\end{aligned}
$$

\footnotetext{
${ }^{3}$ We also include the $\mathbf{D}^{4} / 8 m^{3}$ terms since they will be necessary later on.
}

$$
+\frac{c_{3}^{h l(1)}}{8 m_{1}^{2}} g^{2} \sum_{i=1}^{n_{f}} \psi^{\dagger} \psi \bar{q}_{i} \gamma_{0} q_{i}+\frac{c_{4}^{h l(1)}}{8 m_{1}^{2}} g^{2} \sum_{i=1}^{n_{f}} \psi^{\dagger} \sigma^{k} \psi \bar{q}_{i} \gamma_{k} \gamma_{5} q_{i}
$$

$$
\begin{aligned}
\mathcal{L}_{\chi}=\text { c.c. of } \mathcal{L}_{\psi}(1 \leftrightarrow 2), \\
\begin{aligned}
\mathcal{L}_{\psi \chi}= & \frac{f_{1}\left({ }^{1} S_{0}\right)}{m_{1} m_{2}} O_{1}\left({ }^{1} S_{0}\right)+\frac{f_{1}\left({ }^{3} S_{1}\right)}{m_{1} m_{2}} O_{1}\left({ }^{3} S_{1}\right)+\frac{f_{8}\left({ }^{1} S_{0}\right)}{m_{1} m_{2}} O_{8}\left({ }^{1} S_{0}\right) \\
& +\frac{f_{8}\left({ }^{3} S_{1}\right)}{m_{1} m_{2}} O_{8}\left({ }^{3} S_{1}\right),
\end{aligned}
\end{aligned}
$$

where

$$
\begin{aligned}
& O_{1}\left({ }^{1} S_{0}\right)=\psi^{\dagger} \chi \chi^{\dagger} \psi, \quad O_{1}\left({ }^{3} S_{1}\right)=\psi^{\dagger} \boldsymbol{\sigma} \chi \chi^{\dagger} \boldsymbol{\sigma} \psi \\
& O_{8}\left({ }^{1} S_{0}\right)=\psi^{\dagger} T^{a} \chi \chi^{\dagger} T^{a} \psi, \quad O_{8}\left({ }^{3} S_{1}\right)=\psi^{\dagger} T^{a} \boldsymbol{\sigma} \chi \chi^{\dagger} T^{a} \boldsymbol{\sigma} \psi
\end{aligned}
$$

The matching coefficients are symmetric under the exchange $m_{1} \leftrightarrow m_{2}, \boldsymbol{\sigma}$ are the Pauli matrices, $i D^{0}=i \partial^{0}$ $-g A^{0}, i \mathbf{D}=i \boldsymbol{\nabla}+g \mathbf{A}, \mathbf{E}^{i}=G^{i 0}, \mathbf{B}^{i}=-\epsilon_{i j k} G^{j k} / 2, \epsilon_{i j k}$ being the usual three-dimensional antisymmetric tensor ${ }^{4}\left[(\mathbf{a} \times \mathbf{b})^{i}\right.$ $\left.\equiv \epsilon_{i j k} \mathbf{a}^{j} \mathbf{b}^{k}\right]$ with $\epsilon_{123}=1$, and c.c. stands for charge conjugate $\left(\psi^{c}=-i \sigma^{2} \chi^{*}, \chi^{c}=i \sigma^{2} \psi^{*}\right.$, and $\left.A_{\mu}^{c}=-A_{\mu}^{T}\right)$.

The NRQCD Lagrangian is defined up to field redefinitions. In the expression adopted here, we have made use of this freedom. Powers larger than one of $i D_{0}$ applied to the quark fields have been eliminated. We have also redefined the gluon fields in such a way that the coefficient in front of $-G^{\mu \nu a} G_{\mu \nu}^{a} / 4$ in $\mathcal{L}_{g}$ is 1 . This turns out to be equivalent to redefining the coupling constant in such a way that it runs with $N_{f}-2=n_{f}$ flavors (for $m_{1}$ $\neq m_{2}, N_{f}-1=n_{f}$ for $m_{1}=m_{2}$ ), where $N_{f}$ are the flavors in QCD (Pineda and Soto, 1998c) [see also Griesshammer (2000) for a calculation of the $\beta$ function in NRQCD]. A possible term $D^{\mu} G_{\mu \alpha}^{a} D_{\nu} G^{\nu \alpha a}$ has been eliminated through the identity (Manohar, 1997)

$$
\begin{gathered}
\int d^{4} x\left(2 D^{\mu} G_{\mu \alpha}^{a} D_{\nu} G^{\nu \alpha a}+2 g f_{a b c} G_{\mu \nu}^{a} G_{\alpha}^{\mu b} G^{\nu \alpha c}\right. \\
\left.+G_{\mu \nu}^{a} D^{2} G^{\mu \nu a}\right)=0 .
\end{gathered}
$$

Finally, a term like $c G_{\mu \nu}^{a} D^{2} G^{\mu \nu a}$ has been eliminated through the field redefinition $A_{\mu} \rightarrow A_{\mu}+2 c\left[D^{\alpha}, G_{\alpha \mu}\right]$ (Pineda and Vairo, 2001).

The Wilson coefficients appearing in the NRQCD Lagrangian will be discussed in Sec. II.D. The $\mathcal{O}\left(1 / \mathrm{m}^{3}\right)$ Lagrangian (without the light-fermion sector) can be found in the paper by Manohar (1997). The Feynman rules associated with the first two lines of Eq. (7) can be found in the article by Bodwin and Chen (1999).

\footnotetext{
${ }^{4}$ In DR several prescriptions are possible for the $\epsilon_{i j k}$ tensors and $\boldsymbol{\sigma}$. Therefore if DR is used, one has to make sure that one uses the same procedure as that used to calculate the matching coefficients.
} 
NR currents should also be considered since they appear in inclusive (electromagnetic) decays, NR sum rules, or $t-\bar{t}$ production near threshold. Similar to the Lagrangian, they can be written as an expansion in $1 / \mathrm{m}$ times some hard matching coefficients times some NR (local) operators. For instance, the electromagnetic vector and axial-vector currents read (see Hoang and Teubner, 1999)

$$
\begin{aligned}
j_{k}^{v}(x)= & b_{1, \mathrm{NR}}^{v}\left(\psi^{\dagger} \sigma_{k} \chi\right)(x)-\frac{b_{2, \mathrm{NR}}^{v}}{6 m^{2}}\left[\psi^{\dagger} \sigma_{k}\left(-\frac{i}{2} \overleftrightarrow{\boldsymbol{D}}\right)^{2} \chi\right](x) \\
& +\cdots \\
j_{k}^{a}(x)= & \frac{b_{1, \mathrm{NR}}^{a}}{m}\left[\psi^{\dagger}\left(-\frac{i}{2} \overleftrightarrow{\boldsymbol{D}} \times \boldsymbol{\sigma}\right)_{k} \chi\right](x)+\cdots
\end{aligned}
$$

where $\psi^{\dagger} \stackrel{\leftrightarrow}{\boldsymbol{D}} \chi \equiv \psi^{\dagger}(\mathbf{D} \chi)-(\mathbf{D} \psi)^{\dagger} \chi$ and the dots stand for corrections, which do not contribute at next-to-next-to leading order (NNLO) for $S$ waves. In practice, most of the physical information can be extracted from the imaginary parts of the four-fermion operators, which are discussed in Sec. II.F.2. In particular, the matching coefficients $b_{1, \mathrm{NR}}^{v}$ and $b_{2, \mathrm{NR}}^{v}$ can be traded for the matching coefficients $\operatorname{Im} f_{\mathrm{EM}}\left({ }^{3} S_{1}\right)$ and $\operatorname{Im} g_{\mathrm{EM}}\left({ }^{3} S_{1}\right)$, respectively.

The QCD Lagrangian is invariant under Lorentz boosts. However, the NR expansion has destroyed the manifest invariance of the EFT under Lorentz boosts. Since the EFT is equivalent to QCD at any order of the strong-coupling and NR expansion, the invariance under Lorentz boosts is not lost but must be somehow incorporated into the EFT. Indeed, it imposes specific constraints on the form of the EFT itself.

Constraints imposed by the relativistic invariance were first worked out for heavy-quark effective theory (HQET), which coincides with NRQCD in the bilinear sector of the heavy-quark fields (Luke and Manohar, 1992; Manohar, 1997). In HQET the realization of the relativistic invariance is called reparametrization invariance. It imposes constraints on the Wilson coefficients of the EFT. For instance,

$$
c_{k}=c_{4}=1, \quad 2 c_{F}-c_{S}-1=0,
$$

where we have dropped the explicit indication of the flavor index.

An alternative derivation consists of imposing the Poincaré algebra on the generators $H, \mathbf{P}, \mathbf{J}$, and $\mathbf{K}$ of time translations, space translations, rotations, and Lorentz-boosts transformations of NRQCD (Brambilla, Gromes, and Vairo, 2003). The idea originates from the work of Dirac (1949), and has been used to constrain the form of the relativistic corrections to phenomenological potentials (Foldy, 1961; Krajcik and Foldy, 1974; Sebastian and Yun, 1979). It was applied to NR EFTs by Brambilla, Gromes, and Vairo (2003) and Vairo (2004a). In a field theory, the Poincaré algebra has to be understood among fields quantized in accordance with the ca- nonical equal-time commutation relations. ${ }^{5}$ The translation and rotation generators $\mathbf{P}$ and $\mathbf{J}$ may be derived from the NRQCD Lagrangian or by matching to the QCD generators. They are exact because translational and rotational invariance have not been explicitly broken in going to the EFT. The Lorentz-boost generators may be obtained by matching order by order in $1 / \mathrm{m}$ to the Lorentz-boost generators of QCD. They depend on some specific matching coefficients independent of those in the Lagrangian. The NRQCD Poincaré generators satisfy the Poincaré algebra if Eq. (14) is satisfied for each flavor up to $\mathcal{O}(1 / \mathrm{m})$ and $\mathcal{O}\left(\boldsymbol{\nabla}^{2} \boldsymbol{\nabla} / \mathrm{m}^{2}\right)$ (plus some other constraints on the matching coefficients appearing in K) (Brambilla, Gromes, and Vairo, 2003). Therefore at the considered order, one gets the same result as from reparametrization invariance. The calculation of constraints specific to NRQCD, i.e., involving four-fermion operators, has not been done in either approach yet. This would correspond to going to higher orders in $1 / \mathrm{m}$.

In general, we shall constrain the matching coefficients of the kinetic energy in accordance with Eq. (14). Occasionally, however, we shall keep them explicit for tracking purposes.

\section{Matching}

The calculation of the NRQCD Wilson coefficients is done through a procedure called matching. In a matching calculation suitable renormalized QCD and renormalized NRQCD Green's functions (or matrix elements) are imposed to be equal for scales below $\nu_{\mathrm{NR}}$ at the desired order of $\alpha_{s}$ and $1 / \mathrm{m}$. In particular, the expansion of Green's functions in external energies $E$ and threemomenta $p$ must be equal. This fixes the matching coefficients, which will depend on the renormalization schemes used in QCD and in NRQCD. It greatly simplifies calculations if these expansions are done before the loop integrals are performed. However, doing so may introduce IR divergences and for the equality between QCD and NRQCD Green's functions to remain valid the same IR regulator must be used in both theories. It is very convenient to use DR as an IR regulator as well as an UV one. This is because all loop integrals in the NRQCD calculations will be scaleless and can be set to zero, as we shall argue below. Let us explain what will happen. Schematically (Manohar, 1997) one has

$$
A_{\text {eff }}\left(\frac{1}{\epsilon_{\mathrm{UV}}}-\frac{1}{\epsilon_{\mathrm{IR}}}\right)
$$

in the EFT, which is zero if $\epsilon_{\mathrm{UV}}=\epsilon_{\mathrm{IR}}$ in DR. Therefore we only have to calculate loop integrals in QCD that depend on a single scale $(m)$. Typically we get

\footnotetext{
${ }^{5}$ More precisely, the algebra imposes relations among the bare fields and coupling constants. These relations are preserved in the renormalized theory if Poincare invariance is not broken by quantum effects.
} 


$$
A \frac{1}{\epsilon_{\mathrm{UV}}}+B \frac{1}{\epsilon_{\mathrm{IR}}}+(A+B) \ln \frac{\nu}{m}+D
$$

Since the full and effective theories share the same IR behavior $B=-A_{\text {eff }}$. Moreover, the UV divergences are absorbed in the coefficients of both theories. In this way the difference between the full and effective theory is

$$
(A+B) \ln \frac{\nu}{m}+D,
$$

which provides the one-loop contribution to the matching coefficients for the effective theory. It is implicit in this procedure that the same renormalization scheme is used for both UV and IR divergences in NRQCD. In the QCD calculation both the UV and IR divergences can also be renormalized in the same way, for instance, using the minimal subtraction scheme, which is the standard one for QCD calculations. This fixes the UV renormalization scheme in NRQCD in which the Wilson coefficients have been calculated and means that for these Wilson coefficients to be consistently used in a NRQCD calculation, it must be carried out in the same scheme, for instance, in dimensional regularization and in the minimal subtraction (MS) scheme.

The matching calculation can be carried out in any gauge since both the QCD and NRQCD Lagrangians are manifestly gauge invariant. However, since most of the times one is matching gauge-dependent Green's functions, the same gauge must be chosen in QCD and NRQCD. Using different gauges or, in general, different ways to carry out the matching procedure, may lead to apparently different results for the matching coefficients (within the same regularization and renormalization scheme). These results must be related by local-field redefinitions. In other words, if both matching calculations had used the same minimal basis of operators, the results would have coincided. If the matching is carried out as described above, it is more convenient to choose a covariant gauge (i.e., Feynman gauge) since only QCD calculations, which are manifestly covariant, are to be carried out.

In the procedure described above, one may worry about the fact that the NR propagator $1 /\left(p^{0}-\mathbf{p}^{2} / m\right)$ contains the scale $m$, which spoils the usual argument (used in HQET, for instance) that loop integrals in the EFT contain no scales once one has expanded in the external energies and three-momenta. Let us argue in the following paragraphs that the procedure is indeed correct.

Consider first the single-quark or single-antiquark sector. In any diagram in NRQCD, one can always choose the momenta flowing along the heavy-quark line in the same direction. Then all heavy-quark propagators will have poles in either the lower or upper half of the complex plane only. Then, if all integrals over the energies flowing through the heavy-quark propagators are carried out by closing the contour around the opposite halfcomplex plane, these energies will be replaced by linear dependencies in the three-momenta in the NR quark propagators. These linear dependencies dominate over quadratic dependencies of the kinetic terms both in the
IR and in the UV. The latter is so because $\nu_{\mathrm{NR}}$ is always smaller than $m$. Hence the kinetic term can be expanded and the integrals become dimensionless. In fact, in DR the kinetic term not only can but must be expanded since this is the only way to ensure that three-momenta remain smaller than $\nu_{\mathrm{NR}}$.

Consider next the quark-antiquark sector. Any fixedorder loop calculation may contain heavy-quarkantiquark irreducible diagrams (meaning diagrams which cannot be disconnected by cutting an internal quark line and an internal antiquark line) and heavyquark-antiquark reducible ones.

Consider first a quark-antiquark irreducible diagram. The fact that at any point of an internal quark propagator there is always at least one gluon propagator (or two light-quark propagators) in addition to an antiquark propagator allows one to choose all momenta flowing both along the quark and along the antiquark propagator in the same direction. Hence the poles of both the quark and antiquark propagators are in the same complex half plane, and therefore the argument put forward for the single-quark sector also holds here.

Consider finally a quark-antiquark reducible diagram. It can always be written as a series of two-particle irreducible (2PI) diagrams linked by a quark and by an antiquark propagator. Let us choose the center-of-mass momentum to be zero and focus on one such 2PI block. If $p\left(p^{\prime}\right)$ is the momentum flowing along the incoming (outgoing) quark line, then $-p\left(-p^{\prime}\right)$ is necessarily the momentum flowing along the incoming (outgoing) antiquark line. $p^{0}\left(p^{0 \prime}\right)$ has two relevant scalings, namely, $p^{0} \sim|\mathbf{p}|$ and $p^{0} \sim \mathbf{p}^{2} / 2 m$. If the scaling $p^{0} \sim|\mathbf{p}|$ occurs, then kinetic terms $\sim \mathbf{p}^{2} / 2 m$ can be neglected in 2PI diagram and no further scale $m$ will be introduced. If the scaling $p^{0} \sim \mathbf{p}^{2} / 2 m$ occurs, then $p^{0}$ can be neglected in the gluon propagators and the only dependence in $p^{0}$ can be reduced to either the quark or antiquark propagator. Furthermore, the internal energies in the 2PI diagram eventually take the value of the three-momenta $|\mathbf{p}|$ and hence $p^{0}$ and $\mathbf{p}^{2} / 2 m$ can be expanded. In either case, no extra scales $m$ are introduced in the 2PI diagrams and they can be set to zero. Consider now the link between two 2PI diagrams. If $p^{0} \sim|\mathbf{p}|$, the kinetic term can be expanded and no further scale $m$ is introduced. If $p^{0} \sim \mathbf{p}^{2} / 2 m$, no further dependence on $p^{0}$ in the 2PI diagrams exists, and hence the integral over $p^{0}$ can be trivially done inducing a $m / \mathbf{p}^{2}$ propagator so that the $m$ dependence factorizes trivially. In summary, two-particle reducible diagrams also become scaleless and can be set to zero.

One might be concerned about the appearance of pinch singularities when the kinetic terms are expanded in the links. Let us show that they are of no concern. Recall first that pinch singularities blow up only after the limit $\eta \rightarrow 0$ is taken, where $i \eta$ defines the causal propagator. We propose taking this limit at the end of the calculation. If no other dependence on $p^{0}$ existed, we could carry out all integrals except the one over $p^{0}$. Since they have no scale, as argued before, and they 
contain no pinch singularities, they can safely be set to zero, and the net result is zero. If there are further dependencies on $p^{0}$, by fraction decomposition one can always isolate the pinch singularity in a term $1 /\left(p^{0}\right.$ $+i \eta)\left(-p^{0}+i \eta\right)$ with no further dependencies on $p^{0}$ (plus other terms with no pinch singularity) and proceed as above.

Let us finally note that this matching procedure corresponds to taking the purely hard contribution in the threshold expansion for the NRQCD matching coefficients.

In order to address the matching calculation, we also need the relation between the QCD and NRQCD quark (antiquark) fields:

$$
\begin{aligned}
& Q_{1}(x) \rightarrow Z_{1}^{1 / 2} \frac{1+\gamma_{0}}{2} e^{-i m_{1} t} \psi(x), \\
& Q_{2}(x) \rightarrow Z_{2}^{1 / 2} \frac{1-\gamma_{0}}{2} e^{i m_{2} t} \chi(x) .
\end{aligned}
$$

At one loop, one obtains for the wave-function renormalization constants

$$
Z_{i}=1+C_{F} \frac{\alpha}{\pi}\left(\frac{3}{4} \ln \frac{m_{i}^{2}}{\nu^{2}}-1\right)+\mathcal{O}\left(\left(\frac{\alpha}{\pi}\right)^{2}\right), \quad i=1,2 .
$$

Notice also that the states are differently normalized in relativistic $\left[\left\langle\mathbf{p} \mid \mathbf{p}^{\prime}\right\rangle=(2 \pi)^{3} 2 \sqrt{\mathbf{p}^{2}+m^{2}} \delta^{3}\left(\mathbf{p}-\mathbf{p}^{\prime}\right)\right]$ or NR $\left[\left\langle\mathbf{p} \mid \mathbf{p}^{\prime}\right\rangle=(2 \pi)^{3} \delta^{3}\left(\mathbf{p}-\mathbf{p}^{\prime}\right)\right]$ theories. Hence in order to compare the $S$-matrix elements between the two theories, a factor $\left(2 \sqrt{\mathbf{p}^{2}+m^{2}}\right)^{1 / 2}$ has to be introduced for each external fermion.

For the single-quark (antiquark) sector as well as for the purely gluonic sector, the matching coefficients have been obtained at one loop up to $\mathcal{O}\left(1 / \mathrm{m}^{2}\right)$ in the background Feynman gauge by Manohar (1997). They read (similarly for $1 \rightarrow 2$ )

$$
\begin{aligned}
c_{F}^{(1)}= & 1+\frac{\alpha_{S}}{2 \pi}\left[C_{F}+\left(1-\ln \frac{m_{1}}{\nu}\right) C_{A}\right], \\
c_{D}^{(1)}= & 1+\frac{\alpha_{S}}{\pi}\left[\left(\frac{8}{3} \ln \frac{m_{1}}{\nu}\right) C_{F}+\left(\frac{1}{2}+\frac{2}{3} \ln \frac{m_{1}}{\nu}\right) C_{A}\right] \\
& -\frac{4}{15} \frac{\alpha_{s}}{\pi} T_{F}\left(\frac{m_{1}^{2}+m_{2}^{2}}{m_{2}^{2}}\right), \\
c_{S}^{(1)}= & 1+\frac{\alpha_{S}}{\pi}\left[C_{F}+\left(1-\ln \frac{m_{1}}{\nu}\right) C_{A}\right], \\
c_{1}^{g(1)}= & \frac{\alpha_{S}}{360 \pi} T_{F}
\end{aligned}
$$

$\left(\nu=\nu_{\mathrm{NR}}\right)$. The complete $\mathcal{O}\left(\alpha_{s}^{2}\right)$ correction to $c_{F}$ is also known (Czarnecki and Grozin, 1997).

For the quark-antiquark sector, they have been obtained at one loop up to $\mathcal{O}\left(1 / \mathrm{m}^{2}\right)$ by Pineda and Soto
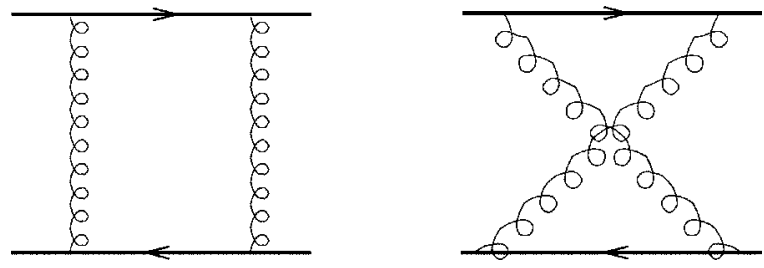

FIG. 1. Relevant one-loop diagrams for the matching of the four-fermion operators at order $\mathcal{O}\left(1 / \mathrm{m}^{2}\right)$ for the case of unequal masses. The incoming and outgoing particles are on shell and exactly at rest.

(1998c). For the nonannihilation diagrams, which are displayed in Fig. 1, it is convenient to use the following basis:

$$
\begin{aligned}
\mathcal{L}_{\psi \chi}= & \frac{d_{s s}}{m_{1} m_{2}} \psi_{1}^{\dagger} \psi_{1} \chi_{2}^{\dagger} \chi_{2}+\frac{d_{s v}}{m_{1} m_{2}} \psi_{1}^{\dagger} \boldsymbol{\sigma} \psi_{1} \chi_{2}^{\dagger} \boldsymbol{\sigma} \chi_{2} \\
& +\frac{d_{v s}}{m_{1} m_{2}} \psi_{1}^{\dagger} T^{a} \psi_{1} \chi_{2}^{\dagger} T^{a} \chi_{2} \\
& +\frac{d_{v v}}{m_{1} m_{2}} \psi_{1}^{\dagger} T^{a} \boldsymbol{\sigma} \psi_{1} \chi_{2}^{\dagger} T^{a} \boldsymbol{\sigma} \chi_{2},
\end{aligned}
$$

which is equivalent to the one in Eq. (8). The relation between them can be found (in four dimensions) in the article by Pineda and Soto (1998c). In this basis, for the case of the quark and antiquark having arbitrary flavor, the matching coefficients at one loop read in the Feynman gauge

$$
\begin{aligned}
d_{s s}= & -C_{F}\left(\frac{C_{A}}{2}-C_{F}\right) \frac{\alpha_{s}^{2}}{m_{1}^{2}-m_{2}^{2}}\left[m_{1}^{2}\left(\ln \frac{m_{2}^{2}}{\nu^{2}}+\frac{1}{3}\right)\right. \\
& \left.-m_{2}^{2}\left(\ln \frac{m_{1}^{2}}{\nu^{2}}+\frac{1}{3}\right)\right], \\
d_{s v}= & C_{F}\left(\frac{C_{A}}{2}-C_{F}\right) \frac{\alpha_{s}^{2}}{m_{1}^{2}-m_{2}^{2}} m_{1} m_{2} \ln \frac{m_{1}^{2}}{m_{2}^{2}} \\
d_{v s}= & -\frac{2 C_{F} \alpha_{s}^{2}}{m_{1}^{2}-m_{2}^{2}}\left[m_{1}^{2}\left(\ln \frac{m_{2}^{2}}{\nu^{2}}+\frac{1}{3}\right)-m_{2}^{2}\left(\ln \frac{m_{1}^{2}}{\nu^{2}}+\frac{1}{3}\right)\right] \\
& +\frac{C_{A} \alpha_{s}^{2}}{4\left(m_{1}^{2}-m_{2}^{2}\right)}\left\{3 \left[m_{1}^{2}\left(\ln \frac{m_{2}^{2}}{\nu^{2}}+\frac{1}{3}\right)\right.\right. \\
& \left.-m_{2}^{2}\left(\ln \frac{m_{1}^{2}}{\nu^{2}}+\frac{1}{3}\right)\right]+\frac{1}{m_{1} m_{2}}\left[m_{1}^{4}\left(\ln \frac{m_{2}^{2}}{\nu^{2}}+\frac{10}{3}\right)\right. \\
& \left.\left.-m_{2}^{4}\left(\ln \frac{m_{1}^{2}}{\nu^{2}}+\frac{10}{3}\right)\right]\right\}, \\
d_{v v}= & \frac{2 C_{F} \alpha_{s}^{2}}{m_{1}^{2}-m_{2}^{2}} m_{1} m_{2} \ln \frac{m_{1}^{2}}{m_{2}^{2}}+\frac{C_{A} \alpha_{s}^{2}}{4\left(m_{1}^{2}-m_{2}^{2}\right)}\left\{\left[m_{1}^{2}\left(\ln \frac{m_{2}^{2}}{\nu^{2}}+3\right)\right.\right. \\
& \left.\left.-m_{2}^{2}\left(\ln \frac{m_{1}^{2}}{\nu^{2}}+3\right)\right]-3 m_{1} m_{2} \ln \frac{m_{1}^{2}}{m_{2}^{2}}\right\}
\end{aligned}
$$

$\left(\nu=\nu_{\mathrm{NR}}\right)$. The $\nu$-independent pieces of $d_{v v}$ depend on the procedure for reducing the $D$-dimensional Dirac matri- 


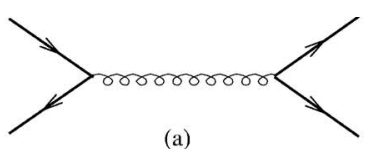

(a)

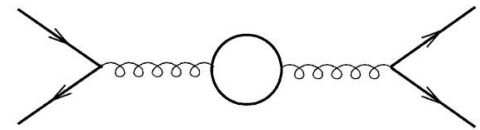

(b)

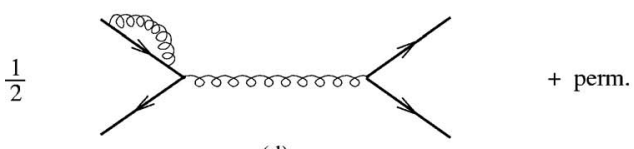

(d)

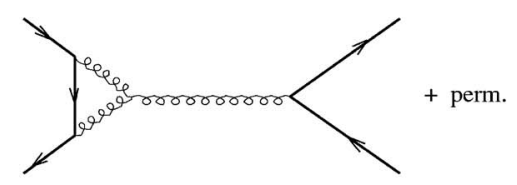

(f)

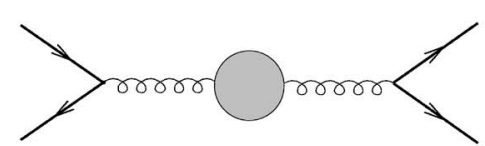

(c)

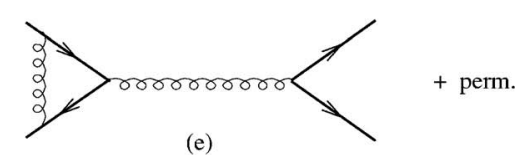

(e)

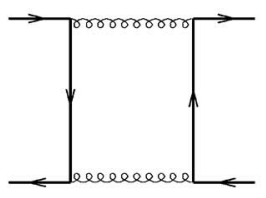

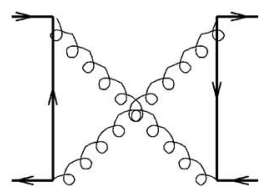

(g)
FIG. 2. Relevant diagrams for the matching of the fourfermion operators at order $\mathcal{O}\left(1 / m^{2}\right)$ and one loop that only appear for the equal-mass case. The incoming and outgoing particles are on shell and exactly at rest. ces to Pauli matrices. Note that we have used the prescription for the dimensionally regulated spin matrices of Pineda and Soto (1998c), which differs from the more standard 't Hooft-Veltman scheme.

The contribution of the diagrams in Fig. 1 to the case of equal flavor is obtained by taking the limit $m_{1} \rightarrow m_{2}$ $=m$. Explicit formulas for this case can be found in the article by Pineda and Soto (1998c). In this case, however, annihilation processes are allowed and they should be taken into account. The relevant annihilation diagrams up to one loop are displayed in Fig. 2.

One obtains

$$
\begin{aligned}
f_{1}\left({ }^{1} S_{0}\right)= & \alpha_{s}^{2} C_{F}\left(\frac{C_{A}}{2}-C_{F}\right)(2-2 \ln 2+i \pi) \\
f_{1}\left({ }^{3} S_{1}\right)= & 0 \\
f_{8}\left({ }^{1} S_{0}\right)= & \frac{\alpha_{s}^{2}}{2}\left(-\frac{3}{2} C_{A}+4 C_{F}\right)(2-2 \ln 2+i \pi) \\
f_{8}\left({ }^{3} S_{1}\right)= & -\pi \alpha_{s}(m)\left(1+\frac{\alpha_{s}}{\pi}\left\{T _ { F } \left[\frac{n_{f}}{3}\left(2 \ln 2-\frac{5}{3}-i \pi\right)\right.\right.\right. \\
& \left.\left.\left.-\frac{8}{9}\right]+\frac{109}{36} C_{A}-4 C_{F}\right\}\right) .
\end{aligned}
$$

Recall that we have to add to the annihilation contributions above the contributions (22)-(25) in the $m_{1} \rightarrow m_{2}$ $=m$ limit. Note that imaginary contributions appear, which are relevant for the calculation of inclusive decay widths. The calculation of corrections of higher order in $\alpha_{s}$ to the imaginary parts of the four-fermion matching coefficients has a long history (Barbieri et al., 1979, 1980, 1981; Hagiwara et al., 1981; Mackenzie and Lepage,
1981; Bodwin et al., 1995; Beneke et al., 1998; Czarnecki and Melnikov, 1998; Petrelli et al., 1998; Maltoni, 1999). An updated list of them and a summary of the state of the art can be found in the article by Vairo (2004b). No further matching calculations beyond the order reported here have been carried out for the real part of fourquark operators.

The $\nu$ dependence of the matching coefficients is eventually traded for a lower scale $\left(|\mathbf{p}|, E, \Lambda_{\mathrm{QCD}}\right)$. This may introduce large logarithms that ought to be summed up, as discussed in Sec. II.E. When higherorder terms in $\alpha_{s}$ are calculated, it may occur that large numerical factors lead to poor convergence of the perturbative series. This is often related to so-called renormalon singularities, which are discussed in Sec. V.

\section{E. Renormalization group}

Once the EFT has been built, one may try to work out the RG improvement of the matching coefficients. This has proven to be a nontrivial task for NRQCD, which is related to the fact that different kinds of degrees of freedom are encoded in the same fields. In other words, the soft and US physics have not been disentangled at the NRQCD Lagrangian level. This means that obtaining the RG improvement at the NRQCD level becomes an ill-defined problem. We shall see later on that the introduction of pNRQCD, which does factorize soft and US physics, indicates how this problem must be posed. Indeed, it is possible to obtain some results at this level (in fact it is even convenient) which will be used afterwards in order to obtain the $\mathrm{RG}$ equations in potential NRQCD (in the weak-coupling regime). The NRQCD matching coefficients are functions of $\nu_{\mathrm{NR}}=\left\{\nu_{p}, \nu_{s}\right\}$. It is convenient to restrict ourselves to the derivation of $R G$ 
equations with respect to the scale $\nu_{s}$ since the RG equations with respect to the scale $\nu_{p}$ are obtained in a much simpler way using pNRQCD.

The matching coefficients of the terms bilinear in the heavy-quark fields and of the pure gluonic terms are just functions of $\nu_{s}$, i.e., $c=c\left(\nu_{s}, m\right) \equiv c\left(\nu_{s}\right)$. This is due to the fact that the UV behavior of the Green's functions in this sector is only sensitive to the energy and not to the three-momentum of the heavy quarks, as can also be seen by explicit computations. Therefore the anomalous dimensions can be computed using the static propagator for the heavy quark, and coincide with those obtained for HQET. The complete leading-logarithm-order (LLO) running of these matching coefficients in the basis of operators (5)-(7) was calculated by Bauer and Manohar (1998) in the (background) Feynman gauge [some partial previous results already existed in the literature (Eichten and Hill, 1990; Falk et al., 1991; Blok et al., 1997)]. For the case of the only nontrivial matching coefficient at $\mathcal{O}(1 / m), c_{F}$, a next-to-leading-logarithmorder (NLLO) evaluation is also available (Amoros et al., 1997; Czarnecki and Grozin, 1997), which we explicitly display to illustrate the typical structure of the RGimproved matching coefficients:

$$
\begin{aligned}
c_{F}\left(m_{i}\right)= & z^{-\gamma_{0} / 2}\left[1+\frac{\alpha_{s}\left(\nu_{h}\right)}{4 \pi}\left(c_{1}+\frac{\gamma_{0}}{2} \ln \frac{\nu_{h}^{2}}{m_{i}^{2}}\right)\right. \\
& \left.+\frac{\alpha_{s}\left(\nu_{h}\right)-\alpha_{s}\left(\nu_{s}\right)}{4 \pi}\left(\frac{\gamma_{1}}{2 \beta_{0}}-\frac{\gamma_{0} \beta_{1}}{2 \beta_{0}^{2}}\right)+\cdots\right],
\end{aligned}
$$

where $z=\left[\alpha_{s}\left(\nu_{s}\right) / \alpha_{S}\left(\nu_{h}\right)\right]^{1 / \beta_{0}}, \nu_{h} \sim m_{i}$ is the hard matching scale, $c_{1}=2\left(C_{A}+C_{F}\right)$, and the one- and two-loop anomalous dimensions are

$$
\gamma_{0}=2 C_{A}, \quad \gamma_{1}=\frac{68}{9} C_{A}^{2}-\frac{52}{9} C_{A} T_{F} n_{f} .
$$

Complications appear when the four-heavy-quark operators $\{f\}$ are considered. As we have mentioned, they depend on both cutoffs: $\nu_{p}$ and $\nu_{s}$. Nevertheless, at one loop, all the dependence of the matching coefficients is due only to $\nu_{s}$, i.e., $f\left(\nu_{p}, \nu_{s}, m\right) \equiv f\left(\nu_{p}, \nu_{s}\right) \simeq f\left(\nu_{s}\right)$. The dependence on $\nu_{p}$ appears at two loops or higher and will be discussed in Sec. IV. In any case, if one restricts oneself to the purely soft running (i.e., $\nu_{s}$ dependence only), it still makes sense to consider the (soft) RG running of the NRQCD matching coefficients including the fourheavy-fermion operators. In this approximation, one can always perform the computation with static propagators for the heavy quarks and order by order in $1 / \mathrm{m}$.

Formally, we can write the NRQCD Lagrangian as an expansion in $1 / \mathrm{m}$ :

$$
\mathcal{L}_{\mathrm{NRQCD}}=\sum_{n=0}^{\infty} \frac{1}{m^{n}} \lambda_{n} O_{n}
$$

where the RG equations of the matching coefficients are

$$
\nu_{s} \frac{d}{d \nu_{s}} \lambda=B_{\lambda}(\lambda) .
$$

The RG equations have a triangular structure (the standard structure one can see, for instance, in HQET RG equations):

$$
\begin{aligned}
& \nu_{s} \frac{d}{d \nu_{s}} \lambda_{0}=B_{0}\left(\lambda_{0}\right), \\
& \nu_{s} \frac{d}{d \nu_{s}} \lambda_{1}=B_{1}\left(\lambda_{0}\right) \lambda_{1}, \\
& \nu_{s} \frac{d}{d \nu_{s}} \lambda_{2}=B_{2(2,1)}\left(\lambda_{0}\right) \lambda_{2}+B_{2(1,2)}\left(\lambda_{0}\right) \lambda_{1}^{2}, \\
& \ldots,
\end{aligned}
$$

where the different $B$ 's can be expanded into a power series in $\lambda_{0}\left[\lambda_{0}\right.$ corresponds to the marginal operators (renormalizable interactions)]. For NRQCD we have $\lambda_{0}=\alpha_{s}$ and $\lambda_{1}=\left\{c_{k}, c_{F}\right\}, \lambda_{2}=\left\{c_{1}^{g}, c_{D}, c_{S},\left\{c^{l l}\right\},\left\{c^{h l}\right\},\{f\}\right\}$.

As we have already mentioned, the LLO running for the $\{c\}$ in Feynman gauge can be read off from the results obtained by Bauer and Manohar (1998). The LLO running of the $\{f\}$ in Feynman gauge can be found in the article by Pineda (2002b).

At this stage, we would like to stress that we are working in a nonminimal basis of operators for the NRQCD Lagrangian. Consequently the values of (some of) the matching coefficients are ambiguous (only some combinations with physical meaning are unambiguous) and could depend upon the gauge in which the calculation has been performed. At the practical level, this means that they will depend on the specific basis of operators we have taken for the NRQCD Lagrangian and on the procedure used (in particular on the gauge). Therefore if working in a nonminimal basis, one should be careful to do the matching using the same gauge for all the operators (or at least for those that are potentially ambiguous). This affects the running of $c_{D}, f_{8}\left({ }^{1} S_{0}\right)$, and $c_{1}^{h l}$. Indeed, it has been shown by Bauer and Manohar (1998) that $c_{D}$ can be absorbed into $f_{8}\left({ }^{1} S_{0}\right)$ and $c_{1}^{\text {hl }}$ by using the equations of motion $\left[\mathbf{D} \cdot \mathbf{E}^{a}=g\left(\psi^{\dagger} T^{a} \psi+\chi^{\dagger} T^{a} \chi\right.\right.$ $\left.\left.+\sum_{j=1}^{n_{f}} \bar{q}_{j} \gamma^{0} T^{a} q_{j}\right)\right]$.

Let us illustrate the point by considering the running of $c_{D}$ and $f_{8}\left({ }^{1} S_{0}\right)$ in the equal-mass case and without light quarks. In Feynamn gauge we obtain

$\nu_{s} \frac{d}{d \nu_{s}} c_{D}=\frac{\alpha_{s}}{4 \pi}\left[\frac{4 C_{A}}{3} c_{D}-\left(\frac{2 C_{A}}{3}+\frac{32 C_{F}}{3}\right) c_{k}^{2}-\frac{10 C_{A}}{3} c_{F}^{2}\right]$,

$\nu_{s} \frac{d}{d \nu_{s}} f_{8}\left({ }^{1} S_{0}\right)=4\left(C_{F}-C_{A}\right) \alpha_{s}^{2} c_{k}^{2}+\frac{3}{2} \alpha_{s}^{2} C_{A} c_{D}$,

while in Coulomb gauge we have 


$$
\begin{aligned}
\nu_{s} \frac{d}{d \nu_{s}} c_{D}(\text { Coulomb })= & \frac{\alpha_{s}}{4 \pi}\left[\frac{22 C_{A}}{3} c_{D}-\left(\frac{32 C_{A}}{3}\right.\right. \\
& \left.\left.+\frac{32 C_{F}}{3}\right) c_{k}^{2}-\frac{10 C_{A}}{3} c_{F}^{2}\right], \\
\nu_{s} \frac{d}{d \nu_{s}} f_{8}\left({ }^{1} S_{0}\right)(\text { Coulomb }) & =\left(4 C_{F}-\frac{3 C_{A}}{2}\right) \alpha_{s}^{2} c_{k}^{2} .
\end{aligned}
$$

Clearly, the running of $c_{D}$ and $f_{8}\left({ }^{1} S_{0}\right)$ is gauge dependent, but the running of the combination $\alpha_{S} c_{D}$ $+(1 / \pi) f_{8}\left({ }^{1} S_{0}\right)$ is not, reflecting the fact that $c_{D}$ can be absorbed into $f_{8}\left({ }^{1} S_{0}\right)$ by means of a suitable field redefinition.

\section{F. Applications: spectrum and inclusive decay widths}

NRQCD has been applied over the last 12 years to a large number of observables related to heavyquarkonium physics. Here we shall briefly discuss two kinds of observables: spectra and inclusive decay widths. Concerning the spectra, we shall mention the state of the art relevant to the lattice determination of the bottomonium levels. We shall keep a continuum EFT point of view since a discussion of lattice NRQCD is beyond the scope of the present work [see Kronfeld (2004) and Lepage (2005) for some recent reviews]. We shall, however, give a more detailed discussion of the inclusive decay width. We have chosen these observables because they are amenable to rather clean theoretical derivations. They will also be addressed in the following sections dedicated to pNRQCD.

Before proceeding, we have to establish a power counting for NRQCD. As was mentioned in Sec. II.B, since the scales $\left(E,|\mathbf{p}|, \Lambda_{\mathrm{QCD}}\right)$ remain dynamical, it is not possible to give a homogeneous counting for each operator. In other words, in contrast to pNRQCD, we shall not be able to disentangle the contributions coming from the different scales. In order to be on the safe side, we have to assume the most conservative counting where each operator counts as $(m v)^{d}, d$ being its dimension, with the exception of $i D_{0}$ which counts as $m v^{2}$ ( $v$ $\sim|\mathbf{p}| / m \sim E /|\mathbf{p}|){ }^{6}$ To count matrix elements of color singlet operators between quarkonium states is rather simple. Since the quarkonium states are normalized, it is sufficient to count the dimension of the gluon field operators and covariant derivatives. For color octet operators, ${ }^{7}$ one has to take into account that they give a nonvanishing contribution between quarkonium states if at least two extra $1 / \mathrm{m}$ operators are inserted. Hence

\footnotetext{
${ }^{6}$ In principle, at least another scale is relevant for quarkonium: $\sqrt{m \Lambda_{\mathrm{QCD}}}$. Since this scale is larger than $E,|\mathbf{p}|$, and $\Lambda_{\mathrm{QCD}}$, it may, in principle, change our counting. We discuss this in Sec. VII.

${ }^{7}$ This applies to the pure octet content of the octet operators $\left(O_{8}, \mathcal{P}_{8}, \ldots\right)$ defined in Eq. (39), which starting from $\mathcal{O}\left(1 / m^{4}\right)$ may also contain singlet parts.
}

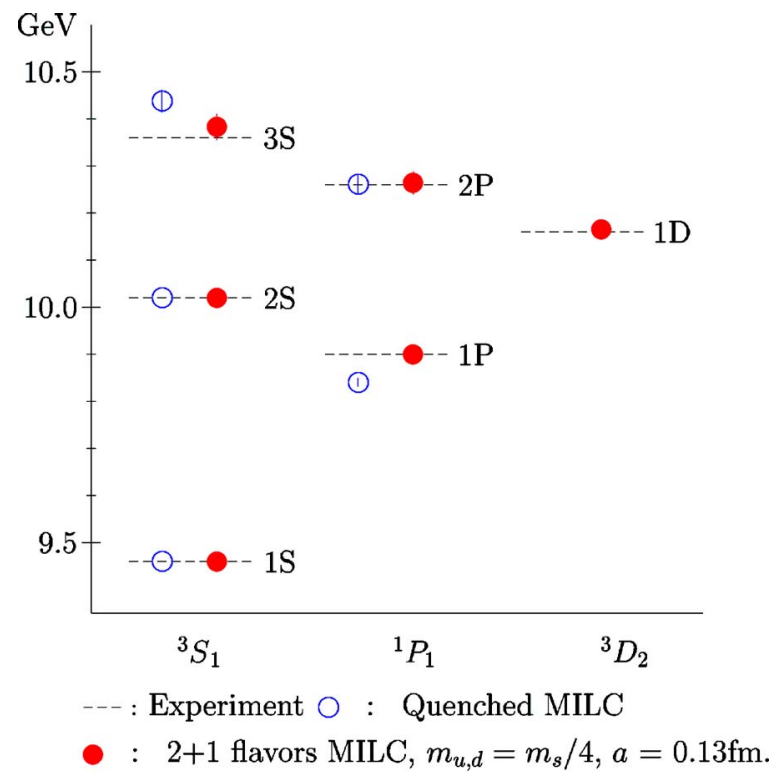

FIG. 3. (Color online) Radial and orbital splittings in the $Y$ system from lattice QCD in the quenched approximation (open circles) and including dynamical $u, d$, and $s$ quarks (filled circles). The lattice spacing has been fixed by the radial splitting between $\Upsilon(2 S)$ and $\Upsilon(1 S)$. The $b$-quark mass has been tuned to get the $Y$ mass correct. From Lepage and Davies, 2004.

using the above rules, one has to add 2 to the dimension of the gluon field operators and covariant derivatives. This counting, which we call the conservative counting, differs from the "original counting" of NRQCD introduced by Lepage et al. (1992). We refer to Sec. II.B for further details.

\section{Spectra}

The idea to put NRQCD on the lattice was a very early one (Lepage et al., 1992). The advantages with respect to full QCD are obvious. The lattice spacing $a$ and the dimension $L$ of the lattice have to fulfill the requirement $1 / a \gg Q \gg q \gg 1 / L$, where $Q$ is the largest and $q$ the smallest scale of the system under study. In full QCD we have $Q \sim m$ while in NRQCD $Q \sim|\mathbf{p}| \ll m$. NRQCD therefore does not require such a fine lattice as full QCD, which means that much more economical simulations are sufficient. The drawback is that the continuum scaling window will not be reached and much more care has to be taken in order to extrapolate from the discrete simulations to the continuum physics.

Some recent results obtained in the lattice version of NRQCD can be found in the article by Lepage and Davies (2004). As a consequence of the rather precise data, for the heavy-quarkonium spectra all levels below the open-flavor threshold have been obtained from multiexponential fits to suitable correlation functions. In Fig. 3 we show some recent quenched and unquenched results for the radial and orbital splittings in the bottomonium system (Gray et al., 2003). 
Here we comment on the theoretical limits of precise lattice results. We neglect all complications and uncertainties connected with the numerical simulations and continuum extrapolations. The version of the NRQCD Lagrangian used in all lattice simulations contains, apart from the Yang-Mills term, only bilinear terms in the heavy-quark fields. The matching coefficients are taken at tree level. This is due to the fact that their calculation in a lattice regularization scheme turns out to be quite cumbersome so that, up to now, only some preliminary numerical estimates are available (Trottier and Lepage, 1998). As a consequence, regardless of how many operators have been added to the bilinear sector of the Lagrangian, the theoretical limit on the precision of the radial splittings is of relative order $\alpha_{s} v^{2} \simeq 0.2 \times 0.1 \simeq 2 \%$ in the original power counting described in the article of Lepage et al. (1992) $\left[\alpha_{s} v \simeq 0.2 \times 0.3 \simeq 6 \%\right.$ in the conservative counting introduced above], while for the fine and hyperfine splittings it is of relative order $\alpha_{s} \simeq 0.2 \simeq 20 \%$. We have assumed for the bottomonium case $v^{2} \simeq 0.1$ and $\alpha_{s}\left(m_{b}\right) \simeq 0.2{ }^{8}$ In any case, the precision in the radial splittings is rather good, while it is worse by an order of magnitude in the fine and hyperfine splittings. In the charmonium case, $v^{2} \simeq 0.3$ and $\alpha_{s}\left(m_{c}\right) \simeq 0.35$, which means that the theoretical limit on the precision of the radial splittings is not smaller than $10 \%$ in the original counting (20\% in the conservative counting). In order to improve the present precision, it is therefore crucial to calculate the one-loop corrections to the Wilson coefficients in the NRQCD Lagrangian in a consistent lattice regularization and renormalization scheme. In this sense, the recent work by Becher and Melnikov (2002, 2003) seems to be rather promising. Note that at order $\alpha_{s} v^{4}\left(\alpha_{s} v^{3}\right.$ in the conservative counting), $1 / \mathrm{m}^{2}$ corrections to the Yang-Mills sector of the NRQCD Lagrangian and four-fermion operators also have to be taken into account.

\section{Inclusive decay widths}

Let us consider heavy quarkonia made out of a quark and an antiquark of the same flavor $\left(m_{1}=m_{2}=m\right)$. Annihilation processes happen in QCD at the scale of the mass $m$. Therefore integrating out these scales in the matching from QCD to NRQCD produces imaginary terms in the matching coefficients of the 4-four-fermion operators of the NRQCD Lagrangian as we have seen in Sec. II.D. Therefore the annihilation width of a heavy quarkonium $H$ into light particles is given by (Bodwin $e t$ al., 1995)

\footnotetext{
${ }^{8}$ It seems too optimistic to replace $\alpha_{s}$ with $\alpha_{s} / \pi$, as suggested by Lepage et al. (1992), since several $\alpha_{s}$ corrections appear with large coefficients [compare with the explicit expressions given in the previous section and with the discussion in the article by Brambilla and Vairo (1999b)]. Moreover, large logarithms could also deteriorate the convergence.
}

$$
\Gamma(H \rightarrow \text { light particles })=2 \operatorname{Im}\left\langle H\left|\mathcal{L}_{\psi \chi}\right| H\right\rangle,
$$

where $|H\rangle$ is a normalized eigenstate of the NRQCD Hamiltonian with the quantum numbers of the considered quarkonium in its center-of-mass frame. ${ }^{9}$ In Eq. (8) we have given $\mathcal{L}_{\psi \chi}$ up to order $1 / \mathrm{m}^{2}$, here we need it up to order $1 / m^{4}$ :

$$
\begin{aligned}
\mathcal{L}_{\psi \chi}= & \frac{f_{1}\left({ }^{1} S_{0}\right)}{m^{2}} O_{1}\left({ }^{1} S_{0}\right)+\frac{f_{1}\left({ }^{3} S_{1}\right)}{m^{2}} O_{1}\left({ }^{3} S_{1}\right) \\
& +\frac{f_{8}\left({ }^{1} S_{0}\right)}{m^{2}} O_{8}\left({ }^{1} S_{0}\right)+\frac{f_{8}\left({ }^{3} S_{1}\right)}{m^{2}} O_{8}\left({ }^{3} S_{1}\right) \\
& +\frac{f_{1}\left({ }^{1} P_{1}\right)}{m^{4}} O_{1}\left({ }^{1} P_{1}\right)+\frac{f_{1}\left({ }^{3} P_{0}\right)}{m^{4}} O_{1}\left({ }^{3} P_{0}\right) \\
& +\frac{f_{1}\left({ }^{3} P_{1}\right)}{m^{4}} O_{1}\left({ }^{3} P_{1}\right)+\frac{f_{1}\left({ }^{3} P_{2}\right)}{m^{4}} O_{1}\left({ }^{3} P_{2}\right) \\
& +\frac{g_{1}\left({ }^{1} S_{0}\right)}{m^{4}} \mathcal{P}_{1}\left({ }^{1} S_{0}\right)+\frac{g_{1}\left({ }^{3} S_{1}\right)}{m^{4}} \mathcal{P}_{1}\left({ }^{3} S_{1}\right) \\
& +\frac{g_{1}\left({ }^{3} S_{1},{ }^{3} D_{1}\right)}{m^{4}} \mathcal{P}_{1}\left({ }^{3} S_{1},{ }^{3} D_{1}\right) \\
& +\left[O_{1} \rightarrow O_{8}, \mathcal{P}_{1} \rightarrow \mathcal{P}_{8}, f_{1} \rightarrow f_{8}, g_{1} \rightarrow g_{8}\right],
\end{aligned}
$$

where the explicit expressions for the operators in the first line can be found in Eq. (10) and for the remaining operators in the paper by Bodwin et al. (1995).

The NRQCD factorization formula for the inclusive heavy-quarkonium annihilation width into light particles reads $\left(d_{n}\right.$ denotes the dimension of the generic fourfermion operator $\left.O^{(n)}\right)$

$$
\begin{gathered}
\Gamma(H \rightarrow \mathrm{LH})=\sum_{n} \frac{2 \operatorname{Im} f^{(n)}}{m^{d_{n}-4}}\left\langle H\left|O^{(n)}\right| H\right\rangle, \\
\Gamma(H \rightarrow \mathrm{EM})=\sum_{n} \frac{2 \operatorname{Im} f_{\mathrm{EM}}^{(n)}}{m^{d_{n}-4}}\left\langle H\left|O_{\mathrm{EM}}^{(n)}\right| H\right\rangle,
\end{gathered}
$$

where we have distinguished between electromagnetic decay widths and decay widths into light hadrons (LH). The information needed in order to describe decays into hard electromagnetic particles is encoded in the electromagnetic contributions to the matching coefficients that we denote by $f_{\mathrm{EM}}, g_{\mathrm{EM}}, \ldots$. We do not use a special symbol to denote the purely hadronic component of the matching coefficients, which is the dominant one. The purely electromagnetic component of the inclusive decay width may be singled out by projecting the fourfermion operators onto the QCD vacuum state $\mid$ vac $\rangle$ according to $\psi^{\dagger} \cdots \chi \chi^{\dagger \cdots} \cdots \psi \rightarrow \psi^{\dagger} \cdots \chi|\operatorname{vac}\rangle\langle\operatorname{vac}| \chi^{\dagger \cdots} \psi$. The

\footnotetext{
${ }^{9}$ This expression only holds at leading order (LO) in the imaginary terms. The exact expression, which has not been necessary for applications so far, reads $\Gamma(H \rightarrow$ light particles $)$ $=-2 \operatorname{Im}(\langle\tilde{H}|H| H\rangle /\langle\tilde{H} \mid H\rangle)$, where $H$ is the NRQCD Hamiltonian and $|\tilde{H}\rangle(\neq|H\rangle$ in general $)$ is the corresponding eigenstate of $H^{\dagger}$.
} 
projected operators are denoted by $O_{\mathrm{EM}}, \mathcal{P}_{\mathrm{EM}}, \ldots$ For instance, $O_{\mathrm{EM}}\left({ }^{1} S_{0}\right)=\psi^{\dagger} \chi|\mathrm{vac}\rangle\langle\operatorname{vac}| \chi^{\dagger} \psi$. The inclusive annihilation width into light hadrons may be obtained from the full annihilation width by switching off the electromagnetic interaction. The factorization formulas (40) and (41) have been rigorously proven and shown diagrammatically in the article by Bodwin et al. (1995).

Working out Eqs. (40) and (41), the explicit expressions for the decay widths of $S$ - and $P$-wave quarkonium up to $\mathcal{O}\left(\operatorname{Im} f \times m v^{5}\right)$ are

$$
\begin{aligned}
\Gamma\left(V_{Q}(n S) \rightarrow \mathrm{LH}\right) \\
=\frac{2}{m^{2}}\left(\operatorname{Im} f_{1}\left({ }^{3} S_{1}\right)\left\langle V_{Q}(n S)\left|O_{1}\left({ }^{3} S_{1}\right)\right| V_{Q}(n S)\right\rangle\right. \\
\quad+\operatorname{Im} f_{8}\left({ }^{3} S_{1}\right)\left\langle V_{Q}(n S)\left|O_{8}\left({ }^{3} S_{1}\right)\right| V_{Q}(n S)\right\rangle \\
\quad+\operatorname{Im} f_{8}\left({ }^{1} S_{0}\right)\left\langle V_{Q}(n S)\left|O_{8}\left({ }^{1} S_{0}\right)\right| V_{Q}(n S)\right\rangle \\
\quad+\operatorname{Im} g_{1}\left({ }^{3} S_{1}\right) \frac{\left\langle V_{Q}(n S)\left|\mathcal{P}_{1}\left({ }^{3} S_{1}\right)\right| V_{Q}(n S)\right\rangle}{m^{2}} \\
\quad+\operatorname{Im} f_{8}\left({ }^{3} P_{0}\right) \frac{\left\langle V_{Q}(n S)\left|O_{8}\left({ }^{3} P_{0}\right)\right| V_{Q}(n S)\right\rangle}{m^{2}} \\
\quad+\operatorname{Im} f_{8}\left({ }^{3} P_{1}\right) \frac{\left\langle V_{Q}(n S)\left|O_{8}\left({ }^{3} P_{1}\right)\right| V_{Q}(n S)\right\rangle}{m^{2}} \\
\left.\quad+\operatorname{Im} f_{8}\left({ }^{3} P_{2}\right) \frac{\left\langle V_{Q}(n S)\left|O_{8}\left({ }^{3} P_{2}\right)\right| V_{Q}(n S)\right\rangle}{m^{2}}\right),
\end{aligned}
$$

$$
\begin{aligned}
\Gamma\left(P_{Q}(n S) \rightarrow \mathrm{LH}\right) \\
=\frac{2}{m^{2}}\left(\operatorname{Im} f_{1}\left({ }^{1} S_{0}\right)\left\langle P_{Q}(n S)\left|O_{1}\left({ }^{1} S_{0}\right)\right| P_{Q}(n S)\right\rangle\right. \\
\quad+\operatorname{Im} f_{8}\left({ }^{1} S_{0}\right)\left\langle P_{Q}(n S)\left|O_{8}\left({ }^{1} S_{0}\right)\right| P_{Q}(n S)\right\rangle \\
\quad+\operatorname{Im} f_{8}\left({ }^{3} S_{1}\right)\left\langle P_{Q}(n S)\left|O_{8}\left({ }^{3} S_{1}\right)\right| P_{Q}(n S)\right\rangle \\
\quad+\operatorname{Im} g_{1}\left({ }^{1} S_{0}\right) \frac{\left\langle P_{Q}(n S)\left|\mathcal{P}_{1}\left({ }^{1} S_{0}\right)\right| P_{Q}(n S)\right\rangle}{m^{2}} \\
\left.\quad+\operatorname{Im} f_{8}\left({ }^{1} P_{1}\right) \frac{\left\langle P_{Q}(n S)\left|O_{8}\left({ }^{1} P_{1}\right)\right| P_{Q}(n S)\right\rangle}{m^{2}}\right),
\end{aligned}
$$

$$
\begin{aligned}
& \Gamma\left(\chi_{Q}(n J S) \rightarrow \mathrm{LH}\right) \\
& =\frac{2}{m^{2}}\left(\operatorname{Im} f_{1}\left({ }^{2 S+1} P_{J}\right) \frac{\left\langle\chi_{Q}(n J S)\left|O_{1}\left({ }^{2 S+1} P_{J}\right)\right| \chi_{Q}(n J S)\right\rangle}{m^{2}}\right. \\
& \left.\quad+\operatorname{Im} f_{8}\left({ }^{2 S+1} S_{S}\right)\left\langle\chi_{Q}(n J S)\left|O_{8}\left({ }^{1} S_{0}\right)\right| \chi_{Q}(n J S)\right\rangle\right),
\end{aligned}
$$

$$
\begin{aligned}
\Gamma\left(V_{Q}(n S) \rightarrow e^{+} e^{-}\right) \\
=\frac{2}{m^{2}}\left(\operatorname{Im} f_{e e}\left({ }^{3} S_{1}\right)\left\langle V_{Q}(n S)\left|O_{\mathrm{EM}}\left({ }^{3} S_{1}\right)\right| V_{Q}(n S)\right\rangle\right. \\
\left.\quad+\operatorname{Im} g_{e e}\left({ }^{3} S_{1}\right) \frac{\left\langle V_{Q}(n S)\left|\mathcal{P}_{\mathrm{EM}}\left({ }^{3} S_{1}\right)\right| V_{Q}(n S)\right\rangle}{m^{2}}\right),
\end{aligned}
$$

$$
\begin{aligned}
\Gamma\left(P_{Q}(n S) \rightarrow \gamma \gamma\right) \\
=\frac{2}{m^{2}}\left(\operatorname{Im} f_{\gamma \gamma}\left({ }^{1} S_{0}\right)\left\langle P_{Q}(n S)\left|O_{\mathrm{EM}}\left({ }^{1} S_{0}\right)\right| P_{Q}(n S)\right\rangle\right. \\
\left.\quad+\operatorname{Im} g_{\gamma \gamma}\left({ }^{1} S_{0}\right) \frac{\left\langle P_{Q}(n S)\left|\mathcal{P}_{\mathrm{EM}}\left({ }^{1} S_{0}\right)\right| P_{Q}(n S)\right\rangle}{m^{2}}\right),
\end{aligned}
$$

$$
\begin{aligned}
& \Gamma\left(\chi_{Q}(n J 1) \rightarrow \gamma \gamma\right) \\
& \quad=2 \operatorname{Im} f_{\gamma \gamma}\left({ }^{3} P_{J}\right) \frac{\left\langle\chi_{Q}(n J 1)\left|O_{\mathrm{EM}}\left({ }^{3} P_{J}\right)\right| \chi_{Q}(n J 1)\right\rangle}{m^{4}}
\end{aligned}
$$$$
\text { for } J=0,2 \text {, }
$$

where the symbols $V$ and $P$ stand for the vector and pseudoscalar $S$-wave heavy quarkonium and the symbol $\chi$ for the generic $P$-wave quarkonium (the states $\chi(n 10)$ and $\chi(n J 1)$ are usually called $h[(n-1) P]$ and $\chi_{J}[(n$ -1) $P]$, respectively).

Looking at Eqs. (42)-(47), the first obvious observation is that in the hadronic decay widths singlet as well as octet matrix elements occur. In the case of the hadronic $P$-wave decay widths the color octet matrix elements are of the same order as the singlet matrix elements. This means that a description of heavy quarkonium in terms of a color-singlet bound state of a heavy quark and antiquark necessarily fails at some point: for $P$-wave decay this point is the leading order! There is another way to understand the role of the octet matrix elements. The singlet matching coefficients are plagued by IR divergences. The coefficients $\operatorname{Im} f\left({ }^{3} P_{0}\right)$ and $\operatorname{Im} f\left({ }^{3} P_{2}\right)$ are IR divergent at next-to-leading order (NLO) (Barbieri et al., 1976). These divergences are precisely canceled by the octet contributions (Bodwin et al., 1992). Therefore the inclusion of the octet matrix elements is crucial to making Eq. (44) physical, i.e., independent of the cutoff. For $S$-wave decays, note that in the original NRQCD power counting used by Lepage et al. (1992) the octet matrix elements are $\mathcal{O}\left(v^{4}\right)$ suppressed compared with the leading order. This is not so within the conservative power counting adopted here, where they are $\mathcal{O}\left(v^{2}\right)$. This may be of phenomenological relevance for $\Gamma(V \rightarrow \mathrm{LH})$ since $\operatorname{Im} f_{1}\left({ }^{3} S_{1}\right)$ is $\mathcal{O}\left(\alpha_{s}\right)$ suppressed with respect to $\operatorname{Im} f_{8}(S)$.

Despite the fact that the NRQCD factorization formulas for inclusive decay widths are theoretically solid and have provided a solution to the long-standing problem of the cancellation of the IR divergences, their practical relevance in calculating inclusive or electromagnetic decay widths of quarkonia has been rather limited. This is mainly due to the following reasons:

(i) NRQCD matrix elements may be fitted on the experimental decay data (Maltoni, 2003) or calculated on the lattice (Bodwin et al., 1996, 2002). The matrix elements of singlet operators can be linked at leading order to the Schrödinger wave functions at the origin (Bodwin et al. 1995) and therefore may be evaluated by means of potential models (Eichten and Quigg, 1995). In general, however, NRQCD matrix elements, in particular of 
higher dimensionality, are poorly known or completely unknown.

(ii) The formulas depend on a large number of matrix elements. In the bottomonium system, $14 S$ - and $P$-wave states lie below the open-flavor threshold $[Y(n S)$ and $\eta_{b}(n S)$ with $n=1,2,3 ; h_{b}(n P)$ and $\chi_{b J}(n P)$ with $n=1,2$ and $J=0,1,2]$ and in the charmonium system $8[\psi(n S)$ and $\eta_{c}(n S)$ with $n=1,2 ; h_{c}(1 P)$ and $\chi_{c J}(1 P)$ with $\left.J=0,1,2\right]$. For these states, Eqs. (42)-(47) describe the decay widths into light hadrons and into photons or $e^{+} e^{-}$in terms of 46 NRQCD matrix elements (40 for the $S$-wave decays and 6 for the $P$-wave decays). More matrix elements are needed if higher-order operators have to be included. Indeed, Ma and Wang (2002) and Bodwin and Petrelli (2002) have stated that higher-order operators not included in Eqs. (42)-(47), even if parametrically suppressed, may turn out to give sizable contributions to the decay widths. This may be the case, in particular, for charmonium, where $v^{2} \sim 0.3$, so that relativistic corrections are large and for $P$-wave decays, where the above formulas provide only the leading-order contribution in the velocity expansion. In fact it was pointed out by Ma and Wang (2002) and Vairo (2002) that if no special cancellations among the matrix elements occur, then the order $v^{2}$ relativistic corrections to the electromagnetic decays $\chi_{c 0} \rightarrow \gamma \gamma$ and $\chi_{c 2} \rightarrow \gamma \gamma$ may be as large as the leading terms. Finally, it was noted by Maltoni (2003) that the relevance of higher-order matrix elements may be enhanced (or suppressed) by the multiplying matching coefficients.

(iii) The convergence of the perturbative series of the four-fermion matching coefficients is often poor [see, for instance, the examples in the article by Vairo (2002)]. This limits, in general, the reliability and stability of the results. Some classes of large perturbative contributions have been resummed for $S$-wave annihilation decays by Braaten and Chen (1998) and Bodwin and Chen (1999, 2001) improving the convergence of the series.

\section{POTENTIAL NRQCD. THE PHYSICAL PICTURE}

Of the full hierarchy of scales in heavy quarkonium, NRQCD takes advantage of the fact that $m$ is much larger than the remaining ones $\left(|\mathbf{p}|, E, \Lambda_{\mathrm{QCD}}, \ldots\right)$ only. This means that if we are interested in physics at the scale of the binding energy $E$, NRQCD contains degrees of freedom that can never appear as physical states at that scale. These are, in particular, light degrees of freedom of energy $\sim|\mathbf{p}| \gg E$ and heavy quarks with energy fluctuations of the same order. Therefore within the philosophy of EFTs, these degrees of freedom should be integrated out. The implementation of this idea gives rise to a new effective theory called pNRQCD (Pineda and Soto, 1998a). The appropriate description of the remaining degrees of freedom and how this integration can actually be carried out will clearly depend on the relative size of $\Lambda_{\mathrm{QCD}}$ compared to the scales $|\mathbf{p}|$ and $E$. We consider the different possibilities in the next two sections. In pNRQCD it is the large scale $|\mathbf{p}|$ that limits the UV cutoff of the energy fluctuations. Even if its typical value in a bound state can be associated with $m v$, its fluctuations may reach up to the scale $m$, which is the UV cutoff for the three-momentum fluctuations of the heavy quarks, $|\mathbf{p}|$.

\section{A. Weak-coupling regime}

If $|\mathbf{p}| \gg \Lambda_{\mathrm{QCD}}$, the integration of degrees of freedom of energy scale $|\mathbf{p}|$ can be done in perturbation theory. Hence we do not expect a qualitative change in the degrees of freedom but only a lowering of their energy cutoff. Let us call the resulting EFT pNRQCD'. pNRQCD' is thus defined by the same particle content as NRQCD and the cutoffs $\nu_{\mathrm{pNR}}=\left\{\nu_{p}, \nu_{u s}\right\}$, where $\nu_{p}$ is the cutoff of the relative three-momentum of the heavy quarks and $\nu_{u s}$ is the cutoff of energy fluctuations of the heavy quarks and of the four-momenta of the gluons and light quarks. They satisfy the following inequalities: $|\mathbf{p}|$ $\ll \nu_{p} \ll m$ and $\mathbf{p}^{2} / m \ll \nu_{u s} \ll|\mathbf{p}|$. The Wilson coefficients of pNRQCD $^{\prime}$ will then depend on $\mathbf{p}$ and $\mathbf{p}^{\prime}$, the threemomenta of the heavy quark and antiquark, respectively, usually through the combination $\mathbf{k}=\mathbf{p}-\mathbf{p}^{\prime}$. Hence nonlocal terms (potentials) in real space are produced. Indeed, these potentials encode the nonanalytic behavior in the momentum transfer $\mathbf{k}$ of the heavy quark, which is of the order of the relative three-momentum of the heavy quarks. This is again a peculiar feature of pNRQCD which had not been observed in any EFT before. It provides an appealing interpretation of the usual potentials in quantum mechanics within an EFT framework.

In order to take advantage of the fact that the threemomentum of the heavy quarks is always larger than the four-momentum of the light degrees of freedom, it is very convenient to use fields in which the relative coordinate (conjugate to the relative momentum) appears explicitly. We define the center-of-mass coordinate of the $Q-\bar{Q}$ system $\mathbf{R} \equiv\left(\mathbf{x}_{1}+\mathbf{x}_{2}\right) / 2$ and the relative coordinate $\mathbf{r} \equiv \mathbf{x}_{1}-\mathbf{x}_{2}$. A $Q-\bar{Q}$ state can be decomposed into a singlet state $S(\mathbf{r}, \mathbf{R}, t)$ and an octet state $O(\mathbf{R}, \mathbf{r}, t)$ in relation to color gauge transformation with respect to the center-of-mass coordinate. (We notice that in QED only the state analogous to the singlet appears.) The gauge fields are evaluated in $\mathbf{R}$ and $t$, i.e., $A_{\mu}=A_{\mu}(\mathbf{R}, t)$. They do not depend on $\mathbf{r}$ due to the fact that, since the typical size of $\mathbf{r}$ is the inverse of the soft scale, gluon fields are multipole expanded with respect to this variable.

If the binding energy $E$ is larger than or of the same order as $\Lambda_{\mathrm{QCD}}$, we have accomplished our goal and the EFT we are looking for, namely, pNRQCD in the weakcoupling regime, coincides with pNRQCD'. If, on the contrary, $\Lambda_{\mathrm{QCD}} \gg E$, we still have to integrate out the energy scale $\Lambda_{\mathrm{QCD}}$ and its associated three-momentum scale $\sqrt{\Lambda_{\mathrm{QCD}} m}$ in order to obtain pNRQCD. This cannot be done perturbatively in $\alpha_{s}$ anymore, but one can definitely continue exploiting the hierarchy of scales, as will be discussed in the following section. 


\section{B. Strong-coupling regime}

For illustration purposes, let us first consider the particular case $|\mathbf{p}| \gg \Lambda_{\mathrm{QCD}} \gg E$, which was discussed in the previous section. We have to figure out what happens to the pNRQCD' degrees of freedom after integrating out those of energy $\sim \Lambda_{\mathrm{QCD}}$. Below the scale $\Lambda_{\mathrm{QCD}}$, it is better to think in terms of hadronic degrees of freedom, which are color-singlet states. Hence the octet field is not acceptable in the final EFT and must be integrated out. Since it couples to gluons of energy $\sim \Lambda_{\mathrm{QCD}}$, it is also expected that it develops a mass gap of the same order. Therefore in pure gluodynamics the only degree of freedom left is the singlet field interacting with a potential, which also has nonperturbative contributions from the integration of degrees of freedom of order $\Lambda_{\mathrm{QCD}}$. In real QCD, pseudo-Goldstone bosons, which have masses smaller than $\Lambda_{\mathrm{QCD}}$, should also be included. These are the expected degrees of freedom of pNRQCD in the strong-coupling regime (Brambilla et al., 2000).

In the general case $|\mathbf{p}| \gtrsim \Lambda_{\mathrm{QCD}}$, we cannot integrate out energy degrees of freedom at the scale $|\mathbf{p}|$ in perturbation theory in $\alpha_{s}$. Still the relevant energy scales are at a lower scale $E \ll|\mathbf{p}| \sim \Lambda_{\mathrm{OCD}}$ and one can in principle build an EFT at that scale, as we have done above in a particular case. This is pNRQCD in the strong-coupling regime. At scales $E \ll \Lambda_{\mathrm{QCD}}, \mathrm{QCD}$ becomes strongly coupled and it is again better to think in terms of hadronic degrees of freedom, which are color-singlet states. Hence the most likely degrees of freedom in this regime are a singlet field and pseudo-Goldstone bosons. This is supported by our knowledge of the static limit of QCD as will be argued below.

In the static limit, there is an energy gap between the ground state and the first excited state. In the nonstatic case there will be a set of states $\left\{n_{u s}\right\}$ whose energies $E_{n_{u s}}$ lie much below the energy of the first excited state in the static case. We denote these states as US. The aim of pNRQCD is to describe the behavior of the US states. Therefore all the physical degrees of freedom in NRQCD with energies larger than $E_{n_{u s}}$ can be integrated out in order to obtain pNRQCD. The available lattice calculations of the static spectrum (see Fig. 4) clearly show that from small to moderately large values of $\mathbf{r}$ there is an energy gap between the ground state and higher excitations. The ground-state energy is known as the static QCD potential. If the binding energy of the heavy-quarkonium state we are interested in is much lower than the first excitation of the static limit, we can integrate out all higher excitations of this limit and keep only the ground state, which will be represented by a singlet field whose static energy is given by the static QCD potential.

Note finally that for heavy-quarkonium states whose binding energy is close to or above the region where higher excitations occur, the use of pNRQCD is not justified and one should stay at the NRQCD level. In the case of real QCD, the heavy-light meson pair threshold plays the role of a higher excitation.

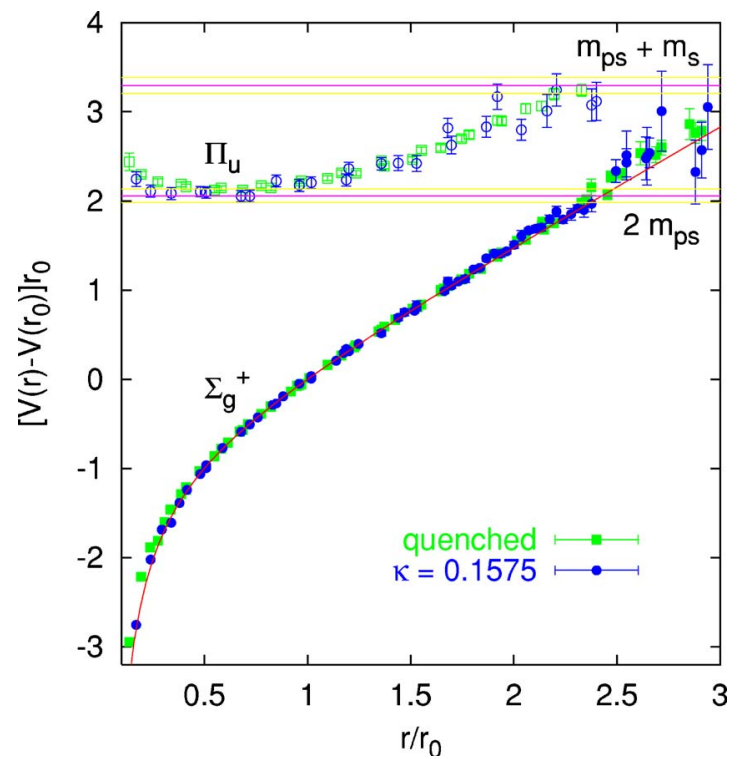

FIG. 4. (Color online) Mass gap between the singlet and hybrid fields. From Bali et al., 2000.

\section{POTENTIAL NRQCD. THE WEAK-COUPLING REGIME}

\section{A. PNRQCD: the degrees of freedom}

The degrees of freedom of pNRQCD in the weakcoupling regime $\left(|\mathbf{p}| \gg E \gtrsim \Lambda_{\mathrm{QCD}}\right)$ are a quark-antiquark pair, gluons, and light quarks with the cutoffs $\nu_{\mathrm{pNR}}$ $=\left\{\nu_{p}, \nu_{u s}\right\} . \quad \nu_{p}$ is the cutoff of the relative threemomentum of the heavy quarks and $\nu_{u s}$ is the cutoff of the energy of the heavy-quark-antiquark pair and of the four-momentum of the gluons and light quarks. They satisfy the following inequalities: $|\mathbf{p}| \ll \nu_{p} \ll m$ and $\mathbf{p}^{2} / m$ $\ll \nu_{u s} \ll|\mathbf{p}|$.

The degrees of freedom of pNRQCD can be represented by the same fields as in NRQCD. The main difference with respect to the NRQCD Lagrangian will be that now nonlocal terms in space (namely, potentials) are allowed. This representation is suitable for explicit perturbative matching calculations. However, in order to establish a power counting, it is more convenient to represent the quark-antiquark pair by a wave-function field

$$
\begin{aligned}
\Psi\left(\mathbf{x}_{1}, \mathbf{x}_{2}, t\right)_{\alpha \beta} \sim & \psi_{\alpha}\left(\mathbf{x}_{1}, t\right) \chi_{\beta}^{\dagger}\left(\mathbf{x}_{2}, t\right) \\
\sim & \frac{1}{N_{c}} \delta_{\alpha \beta} \psi_{\sigma}\left(\mathbf{x}_{1}, t\right) \chi_{\sigma}^{\dagger}\left(\mathbf{x}_{2}, t\right) \\
& +\frac{1}{T_{F}} T_{\alpha \beta}^{a} T_{\rho \sigma}^{a} \psi_{\sigma}\left(\mathbf{x}_{1}, t\right) \chi_{\rho}^{\dagger}\left(\mathbf{x}_{2}, t\right) .
\end{aligned}
$$

This can be rigorously achieved in a NR system: (i) time is universal, and hence one can constrain oneself to calculating correlators in which the time coordinate of the quark field coincides with the time coordinate of the antiquark field; (ii) since particle and antiparticle numbers are separately conserved, if we are interested in the one-heavy-quark one-heavy-antiquark sector, there is no loss of generality if we project our theory to that subspace of the Fock space which is described by the wave- 
function field $\Psi\left(\mathbf{x}_{1}, \mathbf{x}_{2}, t\right)$. Furthermore, this wavefunction field can be uniquely decomposed into singletand octet-field components with homogeneous (US) gauge transformations with respect to the center-of-mass coordinate:

$$
\begin{aligned}
\Psi\left(\mathbf{x}_{1}, \mathbf{x}_{2}, t\right)= & P\left[e^{i g \int_{\mathbf{x}_{2}}^{\mathbf{x}_{1}} \mathbf{A} \cdot d \mathbf{x}}\right] S(\mathbf{r}, \mathbf{R}, t) \\
& +P\left[e^{i g \int_{\mathbf{R}}^{\mathbf{x}_{1}} \mathbf{A} \cdot d \mathbf{x}}\right] O(\mathbf{r}, \mathbf{R}, t) P\left[e^{i g \int_{\mathbf{x}_{2}}^{\mathbf{R}} \mathbf{A} \cdot d \mathbf{x}}\right] .
\end{aligned}
$$

$P$ stands for path ordered. Under (US) color gauge transformations $[g(\mathbf{R}, t)]$, we have

$$
\begin{aligned}
& S(\mathbf{r}, \mathbf{R}, t) \rightarrow S(\mathbf{r}, \mathbf{R}, t), \\
& O(\mathbf{r}, \mathbf{R}, t) \rightarrow g(\mathbf{R}, t) O(\mathbf{r}, \mathbf{R}, t) g^{-1}(\mathbf{R}, t) .
\end{aligned}
$$

Using these fields has the advantage that the relative coordinate $\mathbf{r}$ is explicit, and the fact that $\mathbf{r}$ is much smaller than the typical length of the light degrees of freedom can be easily implemented via a multipole expansion. This implies that the gluon fields will always appear evaluated at the center-of-mass coordinate. Note that this is nothing but translating to real space the constraint $\nu_{p} \gg \nu_{u s}$. In addition, if we restrict ourselves to the singlet field only, we are left with a theory which is totally equivalent to a quantum-mechanical Hamiltonian. The whole theory, however, will contain singlet-to-octet transitions mediated by the emission of an US gluon, which cannot be encoded in any quantum-mechanical Hamiltonian.

\section{B. Power counting}

The power counting of the pNRQCD Lagrangian is easier to establish when it is written in terms of singlet and octet fields. Since quark and antiquark particle numbers are separately conserved, the Lagrangian will be bilinear in these fields and we only have to estimate the size of the terms multiplying those bilinears. $m$ and $\alpha_{s}(m)$, inherited from the hard matching coefficients, have well-known values. Derivatives with respect to the relative coordinate $i \nabla_{\mathrm{r}}$ and $1 / r \sim k$ (the transfer momentum) must be assigned the soft scale $\sim|\mathbf{p}|$. Time derivatives $i \partial_{0}$, center-of-mass derivatives $i \nabla_{\mathbf{R}}$, and the fields of the light degrees of freedom must be assigned the US scale $E \sim \mathbf{p}^{2} / m$. The $\alpha_{s}$ arising in the matching calculation from NRQCD, namely, those in the potentials, must be assigned the size $\alpha_{s}(1 / r)$ and those associated with the light degrees of freedom (gluons, at lower orders) the size $\alpha_{s}(E)$. If $\Lambda_{\mathrm{QCD}}$ did not exist (as in QED) this would provide a homogeneous counting in which each term has a well-defined size. If $E \sim \Lambda_{\mathrm{QCD}}$ [recall that then $\left.\alpha_{s}(E) \sim 1\right]$ this is also true, but calculations at the US scale cannot be done in perturbation theory in $\alpha_{s}(E)$ anymore. If $E \gg \Lambda_{\mathrm{QCD}}$, the counting becomes inhomogeneous (i.e., it is not possible to assign a priori a unique size to each term) since the light degrees of freedom may have contributions both at the scale $E$ and at the scale $\Lambda_{\mathrm{QCD}}$ (see Sec. IV.G). Nevertheless, the largest size a term can have can be estimated identically as before.

\section{Lagrangian and symmetries}

The degrees of freedom of pNRQCD can be arranged in several ways and so accordingly can the pNRQCD Lagrangian. We first write it in terms of quarks and gluons, which allows a smooth connection with the NRQCD section. One of the most distinct features of the pNRQCD Lagrangian is the appearance of the terms $V$, nonlocal in $r$, as matching coefficients of fourfermion operators:

$$
\begin{aligned}
L_{\text {pot }}= & -\int d^{3} \mathbf{x}_{1} d^{3} \mathbf{x}_{2} \psi^{\dagger}\left(t, \mathbf{x}_{1}\right) \chi\left(t, \mathbf{x}_{2}\right) V\left(\mathbf{r}, \mathbf{p}_{1}, \mathbf{p}_{2}, \mathbf{S}_{1}, \mathbf{S}_{2}\right) \\
& \times(\text { US gluon fields }) \chi^{\dagger}\left(t, \mathbf{x}_{2}\right) \psi\left(t, \mathbf{x}_{1}\right),
\end{aligned}
$$

where $\mathbf{p}_{j}=-i \boldsymbol{\nabla}_{\mathbf{x}_{i}}$, for $j=1,2$, and $\mathbf{S}_{1}=\boldsymbol{\sigma}_{1} / 2, \mathbf{S}_{2}=\boldsymbol{\sigma}_{2} / 2$ act on the fermion and antifermion, respectively (the fermion and antifermion spin indices are contracted with the indices of $V$, which are not explicitly displayed). Typically, US gluon fields show up at higher order. With this new term the pNRQCD Lagrangian can be written in the following way:

$$
L_{\mathrm{pNRQCD}}=L_{\mathrm{NRQCD}}^{\mathrm{US}}+L_{\mathrm{pot}},
$$

where $L_{\mathrm{NRQCD}}^{\mathrm{US}}$ has the form of the NRQCD Lagrangian but all the gluons must be understood as US. This way of writing the pNRQCD Lagrangian is advantageous for calculating the matching potentials straightforwardly by means of standard Feynman-diagram techniques. On the other hand, for the study of heavy quarkonium, it is convenient, before calculating physical quantities, to project the above Lagrangian onto the quark-antiquark sector of the Fock space. This makes the multipole expansion explicit at the Lagrangian level and it may also be useful at the matching level, depending on how it is done. An example is the matching via Wilson loops discussed in Sec. IV.F. The projection onto the quark-antiquark sector is easily done at the Hamiltonian level by projecting onto the subspace spanned by

$$
\left.\int d^{3} \mathbf{x}_{1} d^{3} \mathbf{x}_{2} \Psi\left(\mathbf{x}_{1}, \mathbf{x}_{2}\right) \psi^{\dagger}\left(\mathbf{x}_{1}\right) \chi\left(\mathbf{x}_{2}\right) \mid \text { US gluons }\right\rangle,
$$

where |US gluons $\rangle$ is a generic state belonging to the Fock subspace with no quarks and antiquarks but an arbitrary number of ultrasoft gluons. The pNRQCD Lagrangian then has the form

$$
\begin{aligned}
L_{\mathrm{pNRQCD}}= & \int d^{3} \mathbf{x}_{1} d^{3} \mathbf{x}_{2} \operatorname{Tr}\left\{\Psi ^ { \dagger } ( t , \mathbf { x } _ { 1 } , \mathbf { x } _ { 2 } ) \left(i D_{0}+\frac{\mathbf{D}_{\mathbf{x}_{1}}^{2}}{2 m_{1}}\right.\right. \\
& \left.\left.+\frac{\mathbf{D}_{\mathbf{x}_{2}}^{2}}{2 m_{2}}+\cdots\right) \Psi\left(t, \mathbf{x}_{1}, \mathbf{x}_{2}\right)\right\} \\
& -\int d^{3} x \frac{1}{4} G_{\mu \nu}^{a}(x) G^{\mu \nu a}(x) \\
& +\int d^{3} x \sum_{i=1}^{n_{f}} \bar{q}_{i}(x) i I D q_{i}(x)+\cdots
\end{aligned}
$$




$$
\begin{aligned}
& +\int d^{3} \mathbf{x}_{1} d^{3} \mathbf{x}_{2} \operatorname{Tr}\left\{\Psi^{\dagger}\left(t, \mathbf{x}_{1}, \mathbf{x}_{2}\right)\right. \\
& \times V\left(\mathbf{r}, \mathbf{p}_{1}, \mathbf{p}_{2}, \mathbf{S}_{1}, \mathbf{S}_{2}\right) \\
& \left.\times(\mathrm{US} \text { gluon fields }) \Psi\left(t, \mathbf{x}_{1}, \mathbf{x}_{2}\right)\right\}
\end{aligned}
$$

where the first four lines stand for the NRQCD Lagrangian projected onto the quark-antiquark sector and

$$
\begin{aligned}
i D_{0} \Psi\left(t, \mathbf{x}_{1}, \mathbf{x}_{2}\right)= & i \partial_{0} \Psi\left(t, \mathbf{x}_{1}, \mathbf{x}_{2}\right)-g A_{0}\left(t, \mathbf{x}_{1}\right) \Psi\left(t, \mathbf{x}_{1}, \mathbf{x}_{2}\right) \\
& +\Psi\left(t, \mathbf{x}_{1}, \mathbf{x}_{2}\right) g A_{0}\left(t, \mathbf{x}_{2}\right)
\end{aligned}
$$

The dots in Eq. (54) stand for higher terms in the $1 / \mathrm{m}$ expansion. The last four lines contain the four-fermion terms specific to pNRQCD. In general US gluon fields may also appear there, but the leading term (in $\alpha_{s}, 1 / m$, and in the multipole expansion) is simply given by the Coulomb law (one gluon exchange):

$$
\frac{\alpha_{S}}{\left|\mathbf{x}_{1}-\mathbf{x}_{2}\right|} \operatorname{Tr}\left[T^{a} \Psi^{\dagger}\left(t, \mathbf{x}_{1}, \mathbf{x}_{2}\right) T^{a} \Psi\left(t, \mathbf{x}_{1}, \mathbf{x}_{2}\right)\right] .
$$

We can enforce that the gluons be US by multipole expanding them in $\mathbf{r}$. In the case of the covariant derivatives in Eq. (54) this corresponds to

$$
\begin{aligned}
i D_{0} \Psi\left(t, \mathbf{x}_{1}, \mathbf{x}_{2}\right)= & i \partial_{0} \Psi\left(t, \mathbf{x}_{1}, \mathbf{x}_{2}\right)-\left[g A_{0}(t, \mathbf{R}), \Psi\left(t, \mathbf{x}_{1}, \mathbf{x}_{2}\right)\right] \\
& -\frac{1}{2} \mathbf{r}^{i}\left(g \partial_{i} A_{0}(t, \mathbf{R})\right) \Psi\left(t, \mathbf{x}_{1}, \mathbf{x}_{2}\right) \\
& -\frac{1}{2} \mathbf{r}^{i} \Psi\left(t, \mathbf{x}_{1}, \mathbf{x}_{2}\right)\left(g \partial_{i} A_{0}(t, \mathbf{R})\right)+\mathcal{O}\left(r^{2}\right), \\
i \mathbf{D}_{\mathbf{x}_{1}} \Psi\left(t, \mathbf{x}_{1}, \mathbf{x}_{2}\right)= & \left(i \nabla_{\mathbf{r}}+\frac{i}{2} \nabla_{\mathbf{R}}+g \mathbf{A}(t, \mathbf{R})\right. \\
& \left.+\frac{\mathbf{r}^{i}}{2}\left(g \partial_{i} \mathbf{A}(t, \mathbf{R})\right)\right) \Psi\left(t, \mathbf{x}_{1}, \mathbf{x}_{2}\right)+\mathcal{O}\left(r^{2}\right) . \\
& \left(-i \nabla_{\mathbf{r}}+\frac{i}{2} \nabla_{\mathbf{R}}\right) \Psi\left(t, \mathbf{x}_{1}, \mathbf{x}_{2}\right) \\
& +\Psi\left(t, \mathbf{x}_{1}, \mathbf{x}_{2}\right)(-g \mathbf{A}(t, \mathbf{R}) \\
& \left.+\frac{\left.\mathbf{r}_{\mathbf{x}_{2}}^{i} g\left(\partial_{i} \mathbf{A}(t, \mathbf{R})\right)\right)+\mathcal{O}\left(r^{2}\right) .}{2}, \mathbf{x}_{1}, \mathbf{x}_{2}\right)= \\
& =(58)
\end{aligned}
$$

From now on, all the gluon (and light-quark) fields will be understood as functions of $t$ and $\mathbf{R}$. We shall not always explicitly display this dependence. According to the power counting given in the previous section, the multipole expansion makes explicit the size of each term in the Lagrangian. On the other hand, expansions like Eqs. (57) and (58) spoil the manifest gauge invariance of the Lagrangian. This may be restored by introducing singlet and octet fields as in Eq. (49). We choose the following normalization with respect to color:

$$
S=S \mathbf{1}_{c} / \sqrt{N_{c}}, \quad O=O^{a} \mathrm{~T}^{a} / \sqrt{T_{F}} .
$$

We shall not always explicitly display their dependence on $\mathbf{R}, \mathbf{r}$, and $t$ in the following. After multipole expansion, the pNRQCD Lagrangian may be organized as an expansion in $1 / m$ and $r$ (and $\alpha_{s}$ ). The most general pNRQCD Lagrangian density, compatible with the symmetries of QCD, that can be constructed with a singlet field, an octet field, and US gluon fields up to order $p^{3} / m^{2}$ (see Sec. IV.B) has the form

$$
\begin{aligned}
\mathcal{L}_{\mathrm{pNRQCD}}= & \int d^{3} \mathbf{r} \operatorname{Tr}\left\{S^{\dagger}\left[i \partial_{0}-h_{s}\left(\mathbf{r}, \mathbf{p}, \mathbf{P}_{\mathbf{R}}, \mathbf{S}_{1}, \mathbf{S}_{2}\right)\right] S\right. \\
& \left.+O^{\dagger}\left[i D_{0}-h_{o}\left(\mathbf{r}, \mathbf{p}, \mathbf{P}_{\mathbf{R}}, \mathbf{S}_{1}, \mathbf{S}_{2}\right)\right] O\right\} \\
& +V_{A}(r) \operatorname{Tr}\left\{O^{\dagger} \mathbf{r} \cdot g \mathbf{E} S+S^{\dagger} \mathbf{r} \cdot g \mathbf{E} O\right\} \\
& +\frac{V_{B}(r)}{2} \operatorname{Tr}\left\{O^{\dagger} \mathbf{r} \cdot g \mathbf{E} O+O^{\dagger} O \mathbf{r} \cdot g \mathbf{E}\right\} \\
& -\frac{1}{4} G_{\mu \nu}^{a} G^{\mu \nu a}+\sum_{i=1}^{n_{f}} \bar{q}_{i} i I D q_{i},
\end{aligned}
$$

$$
\begin{aligned}
h_{s}\left(\mathbf{r}, \mathbf{p}, \mathbf{P}_{\mathbf{R}}, \mathbf{S}_{1}, \mathbf{S}_{2}\right)= & \left\{c_{S}^{(1,-2)}(r), \frac{\mathbf{p}^{2}}{2 m_{\mathrm{red}}}\right\}+c_{S}^{(1,0)}(r) \frac{\mathbf{P}_{\mathbf{R}}^{2}}{2 m_{\mathrm{tot}}} \\
& +V_{s}\left(\mathbf{r}, \mathbf{p}, \mathbf{P}_{\mathbf{R}}, \mathbf{S}_{1}, \mathbf{S}_{2}\right)
\end{aligned}
$$

$$
\begin{aligned}
h_{o}\left(\mathbf{r}, \mathbf{p}, \mathbf{P}_{\mathbf{R}}, \mathbf{S}_{1}, \mathbf{S}_{2}\right)= & \left\{c_{O}^{(1,-2)}(r), \frac{\mathbf{p}^{2}}{2 m_{\mathrm{red}}}\right\}+c_{O}^{(1,0)}(r) \frac{\mathbf{P}_{\mathbf{R}}^{2}}{2 m_{\mathrm{tot}}} \\
& +V_{o}\left(\mathbf{r}, \mathbf{p}, \mathbf{P}_{\mathbf{R}}, \mathbf{S}_{1}, \mathbf{S}_{2}\right)
\end{aligned}
$$

$$
\begin{aligned}
& V_{s}=V_{s}^{(0)}+\frac{V_{s}^{(1,0)}}{m_{1}}+\frac{V_{s}^{(0,1)}}{m_{2}}+\frac{V_{s}^{(2,0)}}{m_{1}^{2}}+\frac{V_{s}^{(0,2)}}{m_{2}^{2}}+\frac{V_{s}^{(1,1)}}{m_{1} m_{2}}, \\
& V_{o}=V_{o}^{(0)}+\frac{V_{o}^{(1,0)}}{m_{1}}+\frac{V_{o}^{(0,1)}}{m_{2}}+\frac{V_{o}^{(2,0)}}{m_{1}^{2}}+\frac{V_{o}^{(0,2)}}{m_{2}^{2}}+\frac{V_{o}^{(1,1)}}{m_{1} m_{2}},
\end{aligned}
$$

where $i D_{0} O \equiv i \partial_{0} O-g\left[A_{0}(\mathbf{R}, t), O\right], \mathbf{P}_{\mathbf{R}}=-i \mathbf{D}_{\mathbf{R}}, \mathbf{p}=-i \boldsymbol{\nabla}_{\mathbf{r}}$, $m_{\text {red }}=m_{1} m_{2} / m_{\text {tot }}$, and $m_{\text {tot }}=m_{1}+m_{2}$. When acting between singlet fields, the color trace reduces $\mathbf{P}_{\mathbf{R}}$ to $-i \boldsymbol{\nabla}_{\mathbf{R}}$. According to the order at which we are working, the potentials have been displayed up to terms of order $1 / m^{2}$. The static and the $1 / m$ potentials are real-valued functions of $r$ only. The $1 / \mathrm{m}^{2}$ potentials have an imaginary part proportional to $\delta^{(3)}(\mathbf{r})$ and a real part that may be decomposed as (we drop the labels $s$ and $o$ for singlet and octet which have to be understood)

$$
\begin{aligned}
& V^{(2,0)}=V_{S D}^{(2,0)}+V_{S I}^{(2,0)}, \quad V^{(0,2)}=V_{S D}^{(0,2)}+V_{S I}^{(0,2),} \\
& V^{(1,1)}=V_{S D}^{(1,1)}+V_{S I}^{(1,1)}, \\
& V_{S I}^{(2,0)}=\frac{1}{2}\left\{\mathbf{p}_{1}^{2}, V_{\mathbf{p}^{2}}^{(2,0)}(r)\right\}+\frac{V_{\mathbf{L}^{2}}^{(2,0)}(r)}{r^{2}} \mathbf{L}_{1}^{2}+V_{r}^{(2,0)}(r),
\end{aligned}
$$




$$
\begin{aligned}
V_{S I}^{(0,2)}= & \frac{1}{2}\left\{\mathbf{p}_{2}^{2}, V_{\mathbf{p}^{2}}^{(0,2)}(r)\right\}+\frac{V_{\mathbf{L}^{2}}^{(0,2)}(r)}{r^{2}} \mathbf{L}_{2}^{2}+V_{r}^{(0,2)}(r), \\
V_{S I}^{(1,1)}= & -\frac{1}{2}\left\{\mathbf{p}_{1} \cdot \mathbf{p}_{2}, V_{\mathbf{p}^{2}}^{(1,1)}(r)\right\}-\frac{V_{\mathbf{L}^{2}}^{(1,1)}(r)}{2 r^{2}} \\
& \times\left(\mathbf{L}_{1} \cdot \mathbf{L}_{2}+\mathbf{L}_{2} \cdot \mathbf{L}_{1}\right)+V_{r}^{(1,1)}(r), \\
V_{S D}^{(2,0)}= & V_{L S}^{(2,0)}(r) \mathbf{L}_{1} \cdot \mathbf{S}_{1}, \\
V_{S D}^{(0,2)=} & -V_{L S}^{(0,2)}(r) \mathbf{L}_{2} \cdot \mathbf{S}_{2}, \\
V_{S D}^{(1,1)}= & V_{L_{1} S_{2}}^{(1,1)}(r) \mathbf{L}_{1} \cdot \mathbf{S}_{2}-V_{L_{2} S_{1}}^{(1,1)}(r) \mathbf{L}_{2} \cdot \mathbf{S}_{1} \\
& +V_{S^{2}}^{(1,1)}(r) \mathbf{S}_{1} \cdot \mathbf{S}_{2}+V_{\mathbf{S}_{12}}^{(1,1)}(r) \mathbf{S}_{12}(\hat{\mathbf{r}}),
\end{aligned}
$$

where $\mathbf{S}_{1}=\boldsymbol{\sigma}_{1} / 2, \mathbf{S}_{2}=\boldsymbol{\sigma}_{2} / 2, \mathbf{L}_{1} \equiv \mathbf{r} \times \mathbf{p}_{1}, \mathbf{L}_{2} \equiv \mathbf{r} \times \mathbf{p}_{2}$, and $\mathbf{S}_{12}(\hat{\mathbf{r}}) \equiv 3 \hat{\mathbf{r}} \cdot \boldsymbol{\sigma}_{1} \hat{\mathbf{r}} \cdot \boldsymbol{\sigma}_{2}-\boldsymbol{\sigma}_{1} \cdot \boldsymbol{\sigma}_{2}$. The pNRQCD Lagrangian density at order $r^{2} / m^{0}, r^{0} / m,(r / m) \mathbf{P}_{\mathbf{R}}$, and $\left(r^{0} / m^{2}\right) \mathbf{P}_{\mathbf{R}}$ and the corresponding matching coefficients at tree level can be found in the article by Brambilla, Gromes, and Vairo (2003).

For the case $m_{1}=m_{2}=m$, the potential has the following structure:

$$
\begin{aligned}
V(r)= & V^{(0)}(r)+\frac{V^{(1)}(r)}{m}+\frac{V^{(2)}}{m^{2}}+\cdots, \\
V^{(2)}= & V_{S D}^{(2)}+V_{S I}^{(2)}, \\
V_{S I}^{(2)}= & \frac{1}{8}\left\{\mathbf{P}_{\mathbf{R}}^{2}, V_{\mathbf{p}^{2}, \mathrm{CM}}^{(2)}(r)\right\}+\frac{\left(\mathbf{r} \times \mathbf{P}_{\mathbf{R}}\right)^{2}}{4 r^{2}} V_{\mathbf{L}^{2}, \mathrm{CM}}^{(2)}(r) \\
& +\frac{1}{2}\left\{\mathbf{p}^{2}, V_{\mathbf{p}^{2}}^{(2)}(r)\right\}+\frac{V_{\mathbf{L}^{2}}^{(2)}(r)}{r^{2}} \mathbf{L}^{2}+V_{r}^{(2)}(r), \\
V_{S D}^{(2)}= & \frac{\left(\mathbf{r} \times \mathbf{P}_{\mathbf{R}}\right) \cdot\left(\mathbf{S}_{1}-\mathbf{S}_{2}\right)}{2} V_{L S, \mathrm{CM}}^{(2)}(r)+V_{L S}^{(2)}(r) \mathbf{L} \cdot \mathbf{S} \\
& +V_{S^{2}}^{(2)}(r) \mathbf{S}^{2}+V_{\mathbf{S}_{12}}^{(2)}(r) \mathbf{S}_{12}(\hat{\mathbf{r}}),
\end{aligned}
$$

$\mathbf{S}=\mathbf{S}_{1}+\mathbf{S}_{2}$ and $\mathbf{L}=\mathbf{r} \times \mathbf{p}$. Other forms of the potential can be brought to the one above by using unitary transformations, or the relation

$$
-\left\{\frac{1}{r}, \mathbf{p}^{2}\right\}+\frac{1}{r^{3}} \mathbf{L}^{2}+4 \pi \delta^{(3)}(\mathbf{r})=-\frac{1}{r}\left(\mathbf{p}^{2}+\frac{1}{r^{2}} \mathbf{r} \cdot(\mathbf{r} \cdot \mathbf{p}) \mathbf{p}\right) .
$$

From Eq. (60) we see that the relative coordinate $\mathbf{r}$ plays the role of a continuous parameter which specifies different fields. Moreover, we note that the Lagrangian is now in an explicitly gauge-invariant form. This is a consequence of the transformation properties (50) of the singlet and octet fields and of the fact that the gluon fields depend on $t$ and $\mathbf{R}$ only. The functions $V_{s}, V_{o}$, $c_{S}^{(1,-2)}, c_{O}^{(1,-2)}, c_{S}^{(1,0)}, c_{O}^{(1,0)}, V_{A}$, and $V_{B}$ are the matching coefficients of the effective theory. At leading order it follows from Eq. (57) that $V_{A}=V_{B}=1$, from Eq. (58) that $c_{S}^{(1,-2)}=c_{O}^{(1,-2)}=c_{S}^{(1,0)}=c_{O}^{(1,0)}=1$, and from Eq. (56) that $V_{s}^{(0)}=-C_{F} \alpha_{s} / r$ and $V_{o}^{(0)}=\left(1 / 2 N_{c}\right) \alpha_{s} / r$.

Equations (52), (54), and (60) provide three different ways to write the pNRQCD Lagrangian. We have also shown how to derive one from the other. This works (and is useful) at tree level. In general, each form of the pNRQCD Lagrangian may be constructed independently of the others by identifying the degrees of freedom, using symmetry arguments, and matching directly to NRQCD.

The expressions for the currents in pNRQCD are equal to those of NRQCD with the replacements: NR $\rightarrow \mathrm{pNR}$ and $\nu \rightarrow \nu_{\mathrm{pNR}}$. In particular, this applies to Eqs. (12) and (13). As in NRQCD, most of the physical information can be extracted from the imaginary part of the potentials, which are proportional to the imaginary part of the NRQCD four-fermion matching coefficients. Therefore the imaginary part of the (singlet or octet) potential will have the following structure (with only local potentials, delta functions, or derivatives of delta functions):

$$
\operatorname{Im} V=\frac{\operatorname{Im} V^{(2)}}{m^{2}}+\frac{\operatorname{Im} V^{(4)}}{m^{4}}+\cdots,
$$

where the explicit expressions for $\operatorname{Im} V^{(2)}$ and $\operatorname{Im} V^{(4)}$ are

$$
\begin{aligned}
\operatorname{Im} V^{(2)}= & -\frac{C_{A}}{2} \delta^{(3)}(\mathbf{r})\left\{4 \operatorname{Im} f_{1}^{\mathrm{pNR}}\left({ }^{1} S_{0}\right)\right. \\
& -2 \mathbf{S}^{2}\left[\operatorname{Im} f_{1}^{\mathrm{pNR}}\left({ }^{1} S_{0}\right)-\operatorname{Im} f_{1}^{\mathrm{pNR}}\left({ }^{3} S_{1}\right)\right] \\
& +4 \operatorname{Im} f_{\mathrm{EM}}^{\mathrm{pNR}}\left({ }^{1} S_{0}\right)-2 \mathbf{S}^{2}\left[\operatorname{Im} f_{\mathrm{EM}}^{\mathrm{pNR}}\left({ }^{1} S_{0}\right)\right. \\
& \left.\left.-\operatorname{Im} f_{\mathrm{EM}}^{\mathrm{pNR}}\left({ }^{3} S_{1}\right)\right]\right\}, \\
\operatorname{Im} V^{(4)}= & C_{A} \mathcal{T}_{S J}^{i j} \nabla_{\mathbf{r}}^{i} \delta^{(3)}(\mathbf{r}) \nabla_{\mathrm{r}}^{j}\left[\operatorname{Im} f_{1}^{\mathrm{pNR}}\left({ }^{2 S+1} P_{J}\right)\right. \\
& \left.+\operatorname{Im} f_{\mathrm{EM}}^{\mathrm{pNR}}\left({ }^{2 S+1} P_{J}\right)\right]+\frac{C_{A}}{2} \Omega_{S J}^{i j}\left\{\nabla_{\mathbf{r}}^{i} \nabla_{\mathbf{r}}^{j}, \delta^{(3)}(\mathbf{r})\right\} \\
& \times\left[\operatorname{Im} g_{1}^{\mathrm{pNR}}\left({ }^{2 S+1} S_{J}\right)+\operatorname{Im} g_{\mathrm{EM}}^{\mathrm{pNR}}\left({ }^{2 S+1} S_{J}\right)\right] \\
\mathcal{T}_{01}^{i j}= & \delta_{i j}\left(2 \mathbf{1}-\mathbf{S}^{2}\right), \\
\mathcal{T}_{10}^{i j}=\frac{1}{3} \mathbf{S}^{i} \mathbf{S}^{j}, & \\
\mathcal{T}_{00}^{i j}= & \delta_{i j}\left(2 \mathbf{1}-\mathbf{S}^{2}\right), \quad \Omega_{11}^{i j}=\delta_{i j} \mathbf{S}^{2}, \\
\mathcal{T}_{12}^{i j}=\left(\frac{\delta_{i k} \mathbf{S}^{\ell}+\delta_{k j \ell}{ }^{\prime} \mathbf{S}^{\ell} \mathbf{S}^{\ell^{\prime}},}{2} \mathbf{S}^{k}\right. & \left.\mathbf{S}^{i} \delta_{k \ell}\right)\left(\frac{\delta_{j k} \mathbf{S}^{\ell}+\delta_{j \ell} \mathbf{S}^{k}}{3}-\frac{\mathbf{S}^{j} \delta_{k \ell}}{3}\right),
\end{aligned}
$$

and we have omitted the labels singlet and octet in the matching coefficients for simplicity. Note that we use a notation for the matching coefficients similar to the one 
used in NRQCD, but this does not imply that the matching coefficients are equal.

The pNRQCD Lagrangian is invariant under charge conjugation plus $1 \leftrightarrow 2$ exchange (82), time reversal (83), and parity (84). In particular singlet, octet, and gluon fields transform under these as

$$
\begin{aligned}
& S(\mathbf{r}, \mathbf{R}, t) \rightarrow \sigma^{2} S(-\mathbf{r}, \mathbf{R}, t)^{T} \sigma^{2}, \\
& O(\mathbf{r}, \mathbf{R}, t) \rightarrow \sigma^{2} O(-\mathbf{r}, \mathbf{R}, t)^{T} \sigma^{2}, \\
& A_{\mu}(\mathbf{R}, t) \rightarrow-A_{\mu}(\mathbf{R}, t)^{T}, \\
& S(\mathbf{r}, \mathbf{R}, t) \rightarrow \sigma^{2} S(\mathbf{r}, \mathbf{R},-t) \sigma^{2}, \\
& O(\mathbf{r}, \mathbf{R}, t) \rightarrow \sigma^{2} O(\mathbf{r}, \mathbf{R},-t) \sigma^{2}, \\
& A_{\mu}(\mathbf{R}, t) \rightarrow A^{\mu}(\mathbf{R},-t), \\
& S(\mathbf{r}, \mathbf{R}, t) \rightarrow-S(-\mathbf{r},-\mathbf{R}, t), \\
& O(\mathbf{r}, \mathbf{R}, t) \rightarrow-O(-\mathbf{r},-\mathbf{R}, t), \\
& A_{\mu}(\mathbf{R}, t) \rightarrow A^{\mu}(-\mathbf{R}, t) .
\end{aligned}
$$

Singlet- and octet-field transformations may be derived from Eq. (48).

The discrete symmetries constrain the form of the Lagrangian. As an example we observe that the charge conjugate of $\int d^{3} \mathbf{r} \operatorname{Tr}\left\{O^{\dagger} \mathbf{r} \cdot g \mathbf{E} O\right\}$ is $\int d^{3} \mathbf{r} \operatorname{Tr}\left\{O^{\dagger} O \mathbf{r} \cdot g \mathbf{E}\right\}$ and therefore only the sum of the two appears in the Lagrangian. For a similar reason the term $\int d^{3} \mathbf{r} \operatorname{Tr}\left\{S^{\dagger}(\mathbf{r}\right.$ $\times \mathbf{p} \cdot g \mathbf{B}) S\} / m$ cannot appear, while the combination $\int d^{3} \mathbf{r} \operatorname{Tr}\left\{O^{\dagger}(\mathbf{r} \times \mathbf{p} \cdot g \mathbf{B}) O-O^{\dagger} O(\mathbf{r} \times \mathbf{p} \cdot g \mathbf{B})\right\} / m$ is possible.

As in NRQCD, the form of the pNRQCD Lagrangian may also be constrained by imposing the Poincaré algebra of the generators $H, \mathbf{P}, \mathbf{J}$, and $\mathbf{K}$ of time translations, space translations, rotations, and Lorentz boosts of the EFT (Brambilla, Gromes, and Vairo, 2003). $H$ is the pNRQCD Hamiltonian. The translation and rotation generators $\mathbf{P}$ and $\mathbf{J}$ may be derived from the pNRQCD Lagrangian or by matching to the NRQCD generators. They are exact because translational and rotational invariance have not been broken in going to the EFT. The Lorentz-boost generators may be obtained by matching to the Lorentz-boost generators of NRQCD. As can be seen from the explicit expressions given by Brambilla, Gromes, and Vairo (2003), they depend on some specific matching coefficient independent of those in the Lagrangian. The tree-level matching may be performed by multipole expanding the NRQCD Lorentz-boost generators and projecting onto singlet and octet twoparticles states. Loop corrections can, in principle, be calculated as has been done for the matching coefficients of the pNRQCD Lagrangian.

Imposing the Poincaré algebra on the above generators constrains the form of the pNRQCD Lagrangian. For the constraints on the Lorentz-boost generators, see Brambilla, Gromes, and Vairo (2003). For the Lagrangian, the constraints

$$
c_{S}^{(1,0)}=c_{O}^{(1,0)}=1
$$

fix the center-of-mass kinetic energy to $\mathbf{P}_{\mathbf{R}}^{2} / 4 m$. The coefficient of the kinetic energy $\mathbf{p}^{2} / m, c_{S}^{(1,-2)}$, is not fixed by Poincaré invariance. However, one may argue that because no other momentum-dependent operator than the kinetic energy of NRQCD, $-\psi^{\dagger} \nabla^{2} /(2 m) \psi+\chi^{\dagger} \nabla^{2} /(2 m) \chi$, may contribute to the kinetic energy of pNRQCD, the coefficients $c_{S}^{(1,0)}$ and $c_{S}^{(1,-2)}$ have to be equal. It follows then that $c_{S}^{(1,-2)}=1$ (analogously for $\left.c_{O}^{(1,-2)}\right){ }^{10}$ In the singlet- and octet-potential sectors we obtain

$$
\begin{aligned}
& \frac{V_{L S, \mathrm{CM}}}{V^{(0) \prime}}=-\frac{1}{2 r}, \quad V_{\mathbf{L}^{2}, \mathrm{CM}}+\frac{r V^{(0) \prime}}{2}=0, \\
& V_{\mathbf{p}^{2}, \mathrm{CM}}+V_{\mathbf{L}^{2}, \mathrm{CM}}+\frac{V^{(0)}}{2}=0,
\end{aligned}
$$

where $V^{\prime}=d V / d r$. We shall come back to the relations between the singlet potentials in the strong-coupling regime in Sec. VII.E.2. Finally, in the singlet-octet and octet-octet sectors of the Lagrangian, the chromoelectric fields are constrained to enter in the combination

$$
\mathbf{r} \cdot\left(g \mathbf{E}+\frac{1}{2}\left\{\frac{\mathbf{P}_{\mathbf{R}}}{2 m} \times, g \mathbf{B}\right\}\right),
$$

i.e., as in the Lorentz force. Further constraints can be found in the article by Brambilla, Gromes, and Vairo (2003).

\section{Feynman rules}

The Feynman rules of $\mathrm{pNRQCD}$ for the static limit were given by Brambilla et al. (2000) in terms of the time variable and background gluon fields. However, for computations in pNRQCD using Feynman diagrams, it is sometimes more useful to consider the Feynman rules in US momentum space (even if preserving the relative distance $\mathbf{r}$ between the heavy quarks in position space). The propagator of the singlet is

$$
\frac{i}{E-h_{s}} \text {. }
$$

This expression contains subleading terms in the velocity expansion. In order to have homogeneous power counting, it is convenient to expand it about the Coulomb Green's function $G_{c}$ defined in Fig. 5, which scales as $1 / m v^{2}$, and similarly for the octet. The complete set of Feynman rules at the order displayed in Eq. (60) is shown in Fig. 5.

\footnotetext{
${ }^{10}$ One may also obtain $c_{S}^{(1,-2)}=1$ by a direct nonperturbative matching computation as done by Brambilla, Pineda, et al. (2001). The relevant steps of that calculation are reproduced in Eqs. (270)-(272). The kinetic-energy operator may be read from the ratio of the $1 / m$ Green's function (272) and the zeroth-order one (270).
} 


$$
\overline{-}=-i G_{c}(E)=\frac{i}{E-h_{s}^{(0)}}=\frac{i}{E-p^{2} / m-C_{F} \alpha_{s} / r}
$$
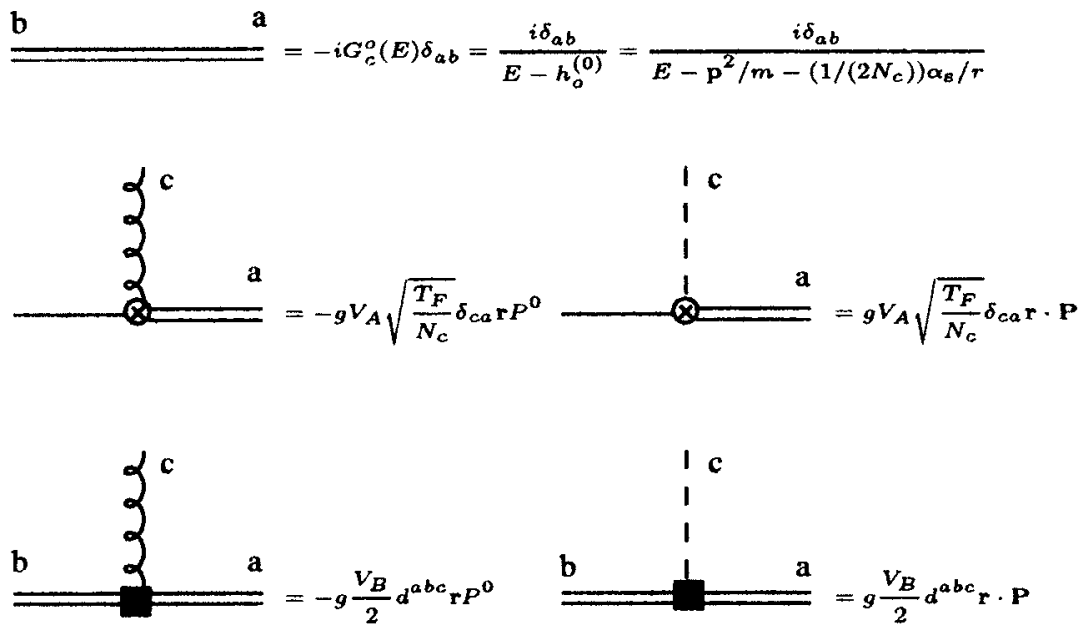
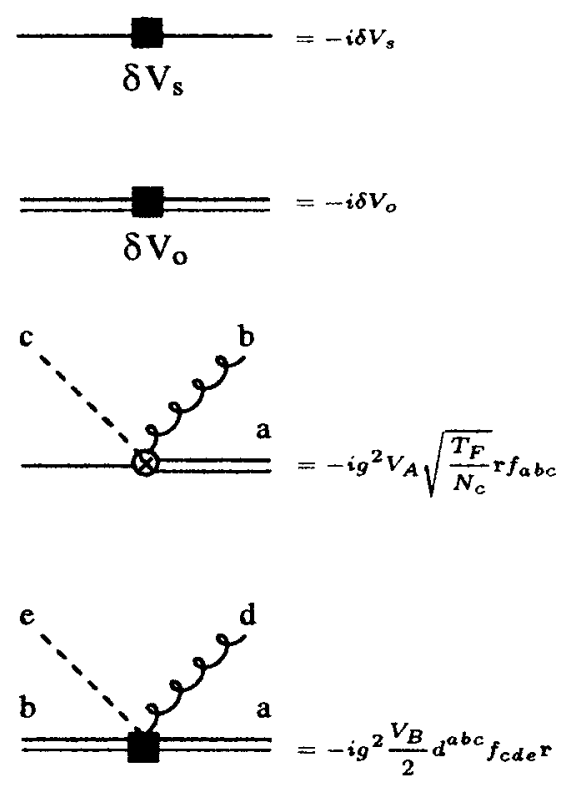

$\delta \mathrm{V}_{\mathrm{o}}$

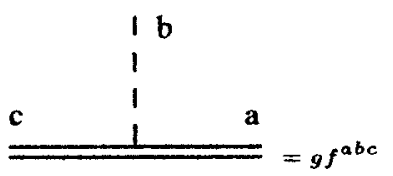

FIG. 5. Propagators and vertices of the pNRQCD Lagrangian (60). Dashed lines represent longitudinal gluons and curly lines transverse gluons. $P^{\mu}$ represents the gluon incoming momentum.

\section{E. Matching: diagrammatic approach}

We discuss here how the matching between NRQCD and pNRQCD [in the formulation of Eq. (52)] within a diagrammatic approach is made along the lines of that shown in Pineda and Soto (1998a, 1998b, 1999). This procedure is especially convenient for obtaining the potentials order by order in $\alpha_{s}$ since the whole technology of Feynman diagrams can be used.

A practical way of obtaining the matching coefficients of pNRQCD is by enforcing that two- and four-fermion Green's functions with arbitrary US external gluons be equal to those of NRQCD at any desired order in $E / k$. It is convenient to expand the energy of the external quark and the energy and momenta of the US gluons around zero before carrying out the loop integrals so that the integrals become homogeneous in the soft scale and hence are easier to evaluate. This may produce IR divergences which are most conveniently (but not necessarily) regulated in DR in the same way as the UV divergences are. Since the IR behavior of NRQCD and pNRQCD is the same, these divergences will cancel out in the matching provided that the same IR regulator is used in both theories. The UV divergences of NRQCD must be renormalized in the MS scheme if we want to use the matching coefficients of the NRQCD Lagrangian computed themselves in this scheme. We still have a choice in the renormalization scheme of pNRQCD. However, it is most advantageous to again use the MS scheme. Indeed, with this choice we can blindly subtract any divergence regardless of whether it is UV or IR in the matching calculation. For the UV divergences of NRQCD and pNRQCD, this just corresponds to our choice of scheme, and for the IR divergences this is possible since as long as we use the same treatment in both theories, their IR behavior is the same. This allows us to set integrals with no scale equal to zero.

Notice that we demand that off-shell Green's functions in NRQCD and pNRQCD be equal and not onshell Green's functions (or on-shell matrix elements) as is usual in many matching calculations, in particular, in matching calculations from QCD to NRQCD. This is due to the fact that we are eventually interested in bound states, and particles in a bound state are typically off shell. More precisely, the equations of motion at lowest order are not those of the free particles. The equations of motion of pNRQCD (with potential terms included) or local-field redefinitions may be consistently used later on to remove time derivatives in higher-order terms and to write the pNRQCD Lagrangian in a standard form, in the philosophy advocated by Scherer and Fearing (1995) [see also Balzereit (1999)]. It has actually been checked by Pineda and Soto (1999) ${ }^{11}$ that this pro-

\footnotetext{
${ }^{11}$ However, there is still some freedom in the choice of the wave-function field due to time-independent unitary transformations which commute with the leading terms in the pNRQCD Lagrangian. Therefore, in general, it is not expected that the standard forms of the pNRQCD Lagrangian calcu-
} 
cedure produces gauge-independent results at $\mathcal{O}\left(m \alpha_{s}^{4}\right)$ in the computation of the positronium spectrum.

The remaining important step for carrying out the matching efficiently is the use of static (HQET) propagators for the fermions. This can be justified as follows. When $p^{0} \sim|\mathbf{p}|$ we are in the kinematical region we wish to integrate out, and the cutoffs of both NRQCD and pNRQCD ensure that the kinetic term $\mathbf{p}^{2} / 2 m$ be subleading with respect to the energy irrespective of the value of $|\mathbf{p}|$. This fact is not automatically implemented in DR. When DR is used, the correct UV behavior of NRQCD is only obtained when expanding about the static propagator. When $p^{0} \sim \mathbf{p}^{2} / 2 m$, we are in a kinematical region which still exists in pNRQCD, and it should not be integrated out. The simplest way to avoid this kinematical region is, again, by expanding the kinetic term. After all these simplifications the computations in the NRQCD side reduce to diagrams with only one scale inside loops. In short, one would have (where $E$ generically denotes the external momentum or the kinetic term $\mathbf{p}^{2} / m$ )

$$
\int d^{D} q f(q, k, E)=\int d^{D} q f(q, k, 0)+\mathcal{O}\left(\frac{E}{k}\right) .
$$

Now we are in a position to prove that no pNRQCD diagram containing a loop contributes to the matching calculation. Consider first the two-fermion Green's function with an arbitrary number of US legs. For potential terms to contribute at least a four-fermion Green's function are needed and hence we only care about US gluons. If we put a momentum $\sim|\mathbf{p}|$ in the fermion line, it cannot flow out through any external US gluon line (by definition of US). Thus it must flow through the fermion line, which is a series of static propagators insensitive to the momentum flowing through them. Upon expanding about external fermion energies and external energies and momenta of the US gluons there is no scale left in any of the integrals and therefore any loop contribution vanishes. In fact, exactly the same argument can be used for the NRQCD calculation. We conclude that the terms bilinear in fermions are exactly the same in NRQCD and $\mathrm{pNRQCD}$. However, we have to keep in mind that the latter (by definition) must be understood as containing ultrasoft gluons only.

Consider next the four-fermion Green's function in pNRQCD containing several potential terms but no US gluons. Since no energy can flow through the potentials and the static propagators are insensitive to the momentum, upon expanding about the US external energy, the integrals over internal energies have no scale. However, these integrals have IR (pinch) singularities which are not regulated by standard DR. How to rigorously deal with them is discussed in Sec. IV.E.1. Since the IR behavior of pNRQCD and NRQCD is the same, the same

lated with different gauges coincide, but that they are only related by one such unitary transformation. This explains the different expressions for the potential that one may find in the literature but which still lead to the same physics. kind of integrals appears in the NRQCD calculation. If we consistently set them to zero, we obtain the correct potential terms. It is important to keep in mind that the Wilson coefficients compensate the different UV behavior of the effective theory (pNRQCD) with respect to that of the more "fundamental" theory (NRQCD). Hence they are not sensitive to the details of the IR behavior, which legitimates the prescription above. Then any loop diagram in pNRQCD with no US gluons can be set to zero. This still holds if an arbitrary number of US gluon lines is included in the diagram. Indeed, any potential line in the diagram may now also contain US momenta from the gluon lines. These, however, can be expanded about zero since they are (by definition) much smaller than the momentum transfer in the potential. Hence the integrals over US gluon energies and momenta contain no scale (again upon expanding the US external energy in the fermion static propagators) and can also be set to zero. In short, loops in PNRQCD will have the following structure in general:

$$
\int d^{D} q f(q, E)=\int d^{D} q f(q, 0)+\mathcal{O}\left(\frac{E}{k}\right)=0 .
$$

In brief, we can directly identify the potential terms from a calculation in NRQCD. We stress again the similarity in the procedure with the matching between QCD and NRQCD as carried out before. The potential terms in $\mathrm{pNRQCD}$ play the role of Wilson coefficients in the matching procedure. As a summary, the final set of rules is the following:

- Compute (off-shell) NRQCD Feynman diagrams within an expansion in $\alpha_{s}, 1 / m$, and $E$. In case loops appear, they have to be computed using static propagators for the heavy quark and antiquark, which makes the integrals depend on $k$ only.

- Match the resulting expression to the tree-level expression in pNRQCD (i.e., the potentials that appear in the pNRQCD Lagrangian) to the required order in $\alpha_{s}, 1 / m$, and $E$.

- Isolate pinch singularities, if they appear, in expressions which are identical to those which appear in the pNRQCD computation and set them to zero. Or, alternatively, one may just subtract the pNRQCD diagrams with the same pinch singularity, as discussed in Sec. IV.E.1.

Let us mention here that when this procedure is used to match local NRQCD four-fermion operators, these do not get any loop correction. Indeed, due to the use of HQET propagators, all NRQCD integrals become scaleless and hence vanish. We often say that they are inherited in pNRQCD.

A word of caution is necessary concerning the procedure above. It relies heavily on the fact that there are no further scales other than $m, k$, and $E$. If, for instance, an energy scale $m^{\prime}$ such that $E \ll m^{\prime} \sim k \ll m$ enters the picture, it would be convenient to take $\nu_{u s} \leqslant m^{\prime}$ rather than $\nu_{u s} \ll k$ and hence $\nu_{p} \ll \sqrt{m m^{\prime}}$ rather than $\nu_{p} \ll m$. Then, in the matching calculation we should also integrate out 
quarks with energy $\sim m^{\prime}$ and three-momentum $\sim \sqrt{m m^{\prime}}$, which cannot be done anymore in the static approximation. A careful analysis of the integration regions along the lines of the threshold expansion discussed below should be carried out in this case. Incidentally, this situation is of physical relevance for the $\mathrm{Y}(1 S)$ system, where the charm-quark mass plays the role of $m^{\prime}$.

It is also possible to perform the matching to pNRQCD using the threshold expansion (Beneke and Smirnov, 1998). This procedure has been followed by several groups (Beneke et al., 1999; Kniehl et al., 2002a, 2002b). Typically (although not always), the procedure consists of taking one specific diagram of NRQCD and splitting it into the different existing regions of momenta. According to this terminology, the modes (and correspondingly the regions of momenta) that appear in NRQCD are the following:

(i) Soft modes. Quarks and gluons with energy and three-momenta of $\mathcal{O}(m v)$ (the quarks are off-shell in this situation).

(ii) Potential modes. Quarks and gluons with energy of $\mathcal{O}\left(m v^{2}\right)$ and three-momenta of $\mathcal{O}(m v)$ (the gluons are off-shell in this situation).

(iii) US modes. Quarks and gluons with energy and three-momenta of $\mathcal{O}\left(m v^{2}\right)$ (in practice, it does not seem there are quarks in this situation).

Integrating out soft modes and potential gluons corresponds to matching NRQCD to pNRQCD. In some cases, it is customary to perform the matching using (free) on-shell quarks. This has the consequence that loops in pNRQCD do not vanish (since the energy is not left as a free parameter in which one can expand) and have to be subtracted accordingly. In addition, the onshell condition may set to zero some terms in the (offshell) potential. When these terms enter into a NRQCD subdiagram of a higher-loop matching calculation, they may give rise to new contributions to the potential due to quark potential loops. This never occurs if the procedure described above is used. In any case, the potentials obtained by using different methods can be related to each other by unitary transformations.

\section{Pinch singularities}

Let us now discuss the issue of the so-called pinch singularity. We illustrate this discussion with the diagram (in the Coulomb gauge) in Fig. 6. Actually, such a diagram appears in the computation of the positronium spectrum at $\mathcal{O}\left(m \alpha^{5}\right)$ carried out in the article by Pineda and Soto (1999). The one-loop integral of this diagram reads
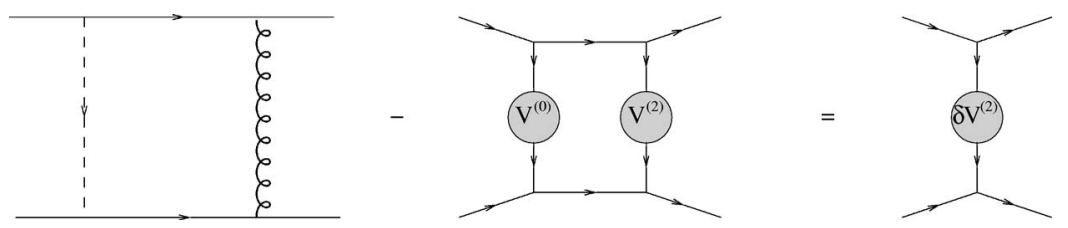

FIG. 7. Matching between NRQCD and pNRQCD taking into account pinch singularities. In pNRQCD the loop regulates the pinch singularity. 


$$
\begin{aligned}
& V_{s}^{(0)}(r)=-C_{F} \frac{\alpha_{V_{s}}(r)}{r}, \\
& V_{s}^{(1)}(r)=-\frac{C_{F} C_{A} D_{s}^{(1)}}{2 r^{2}}, \\
& V_{\mathbf{p}^{2}, s}^{(2)}(r)=-C_{F} D_{1, s}^{(2)}, \\
& V_{\mathbf{L}^{2}, s}^{(2)}(r)=\frac{C_{F} D_{2, s}^{(2)} \frac{1}{r},}{2} \\
& V_{r, s}^{(2)}(r)=\pi C_{F} D_{d, s}^{(2)} \delta^{(3)}(\mathbf{r}), \\
& V_{S^{2}, s}^{(2)}(r)=\frac{4 \pi C_{F} D_{S^{2}, s}^{(2)}}{3} \delta^{(3)}(\mathbf{r}), \\
& V_{L S, s}^{(2)}(r)=\frac{3 C_{F} D_{L S, s}^{(2)} \frac{1}{r^{3}},}{2} \\
& V_{\mathbf{S}_{12}, s}^{(2)}(r)=\frac{C_{F} D_{S_{12}, s}^{(2)} \frac{1}{r^{3}},}{4},
\end{aligned}
$$

where $\alpha_{V_{s}}$ and the various $D$ 's depend logarithmically on $r$ and the renormalization scale $\nu_{\mathrm{pNR}}$. In order to obtain the spectrum at order $m \alpha_{s}^{4}, \alpha_{V_{s}}$ has to be calculated to order $\alpha_{s}^{3}$ (two loops), $V_{s}^{(1)}$ to order $\alpha_{s}^{2}$ (one loop), and the remaining potentials to order $\alpha_{s}$ (tree level). They are

$$
\begin{aligned}
\alpha_{V_{s}}= & \alpha_{s}(r)\left\{1+\left(a_{1}+2 \gamma_{E} \beta_{0}\right) \frac{\alpha_{s}(r)}{4 \pi}+\left[\gamma_{E}\left(4 a_{1} \beta_{0}+2 \beta_{1}\right)\right.\right. \\
& \left.\left.+\left(\frac{\pi^{2}}{3}+4 \gamma_{E}^{2}\right) \beta_{0}^{2}+a_{2}\right] \frac{\alpha_{s}^{2}(r)}{16 \pi^{2}}\right\}, \\
D_{s}^{(1)}= & \alpha_{s}^{2}(r), \\
D_{1, s}^{(2)}= & D_{2, s}^{(2)}=D_{d, s}^{(2)}=D_{S^{2}, s}^{(2)}=D_{L S, S}^{(2)}=D_{S_{12}, s}^{(2)}=\alpha_{s}(r) .
\end{aligned}
$$

$a_{1}$ was computed by Fischler (1977) and $a_{2}$ by Peter (1997) and Schröder (1999b). If one wishes to have the spectrum to one order higher, namely, $m \alpha_{s}^{5}$, all these potentials must be calculated to one more power in $\alpha_{s}$. For $\alpha_{V}$, only the logarithmic contributions are known (Brambilla et al., 1999b; Kniehl and Penin, 1999) [Padé approximant (Chishtie and Elias, 2001) and renormalonbased (Pineda, 2001) estimates are also available]. $V_{s}^{(1)}$ was calculated by Kniehl, Penin, Steinhauser, et al. (2002) (the logarithmic corrections were computed by Brambilla et al., 1999a and Kniehl and Penin, 1999) and the complete $V_{s}^{(2)}$ have been computed over the years (Buchmüller et al., 1981; Gupta and Radford, 1981, 1982; Pantaleone et al., 1986; Titard and Yndurain, 1994; Brambilla et al., 1999a; Pineda and Soto, 1999; Manohar and Stewart, 2000b; Kniehl et al., 2002a) and can be found in the article by Kniehl et al. (2002a). Several com- ments are in order concerning these calculations.

(1) The potentials in the matching calculation appear naturally in momentum space, and so they are given in many of the references above. The real-space potentials, which are better suited for bound-state calculations, are obtained by Fourier transforming the momentum-space potentials. At lower orders, it is enough to take the Fourier transform in three dimensions in the sense of distributions (Titard and Yndurain, 1994). At higher orders, it must be taken in $d$ dimensions, as discussed below.

(2) In different papers, the results displayed for each of the potentials may vary, even if the same basis (72) is used. This does not mean a priori that there are inconsistencies. The basis (72) is overcomplete and hence apparently different results may be related to each other by unitary transformations. In particular, $V_{s}^{(1)}$ can be totally reshuffled into $1 / \mathrm{m}^{2}$ potentials.

(3) In earlier papers, the potentials were calculated directly from QCD without expanding in the kinetic energy. In that case there are contributions from the pNRQCD side to the matching calculation due to the fact that the kinetic term in the pNRQCD Hamiltonian cannot be expanded anymore. In this framework, the integrals involved in the calculation have more than one scale and are harder to evaluate.

(4) In higher-order calculations, quantum-mechanical perturbation theory requires regularization and renormalization. The UV divergences are renormalized by local potentials inherited from NRQCD and the scale dependence is compensated by the one in the NRQCD matching coefficients. In order to use the NRQCD matching coefficients obtained in Sec. II.D, the potentials must be kept in $d$ dimensions. This is not important as far as the soft or US factorization is concerned (it amounts to a change of subtraction scheme), but it becomes when the calculation is sensitive to divergences due to the hard or potential factorization. This occurs at order $m \alpha_{s}^{6}$ for the spectrum and in $\mathcal{O}\left(\alpha_{s}^{2}\right)$ corrections for the current. Note that any loop correction to a given (e.g., Coulomb) potential slightly changes its functional form [it gets multiplied by $(r \nu)^{(4-D)}$ for each loop]. The expressions for the potentials in three dimensions calculated at higher orders display small logarithms, which eventually cancel out in the full calculation, in addition to the large logarithms, which eventually become $\ln \alpha_{s}$, as discussed by Kniehl et al. (2002a) [note that in Brambilla et al. (1999a) only the large logarithms were displayed].

(5) The octet potential is also known at two-loop accuracy (Kniehl et al., 2005),

$$
\begin{aligned}
& V_{o}^{(0)}(r) \equiv\left(\frac{C_{A}}{2}-C_{F}\right) \frac{\alpha_{V_{o}}(r)}{r}, \\
& \alpha_{V_{o}}(r)=\alpha_{V_{s}}(r)-\left(\frac{3}{4}-\frac{\pi^{2}}{16}\right) C_{A}^{2} \alpha_{s}^{3}+\mathcal{O}\left(\alpha_{s}^{4}\right) .
\end{aligned}
$$

(6) At order $m \alpha_{s}^{5}$ for the spectrum and at $\mathcal{O}\left(\alpha_{s}^{3}\right)$ for the current US loops start to contribute. This implies that $V_{A}(r)$ is also needed. At tree level we have 


$$
V_{A}(r)=V_{B}(r)=1 .
$$

(7) For the case $m_{1} \neq m_{2}$, the $1 / m^{2}$ potentials have only been calculated in the scheme described in Sec. IV.E for QED (Pineda and Soto, 1999). Earlier calculations for both QCD (Gupta and Radford, 1982) and QED (Gupta et al., 1989) exist, which have been carried out by matching directly the fundamental theory to a quantum-mechanical Hamiltonian.

(8) RG-improved expressions for the potential can also be obtained. They are discussed in Sec. IV.H.

Finally, we would like to briefly discuss the matching of currents and the imaginary pNRQCD potential. Integrating out the soft scale when matching local currents produces scaleless integrals, which are zero in DR. This means that the matching coefficient remains the same at the matching scale. If we take the electromagneticvector current as an example, the matching condition is $b_{1, \mathrm{pNR}}^{v}\left(\nu_{p}, \nu_{u s}=\nu_{s}\right)=b_{1, \mathrm{NR}}^{v}\left(\nu_{p}, \nu_{s}\right)$. In the case of $b_{1, \mathrm{NR}}^{v}$, only a dependence on $\nu_{p}$ appears (at least at low orders). An equivalent discussion applies to the imaginary terms of the Lagrangian for which the general matching condition $\operatorname{Im} f^{\mathrm{pNR}}\left(\nu_{p}, \nu_{u s}=\nu_{s}\right)=\operatorname{Im} f\left(\nu_{p}, \nu_{s}\right)$ holds. Nevertheless, one should keep in mind that the expressions for the matching coefficients will change once their running is considered (see Sec. IV.H).

\section{F. Matching: Wilson-loop approach}

We discuss here another way to perform the matching to pNRQCD which we sometimes denote as Wilsonloop matching. With respect to the previously discussed procedure, it is characterized by the following points.

(a) It is done in coordinate space.

(b) It is done with the pNRQCD Lagrangian in the form of Eq. (60). This means that the degrees of freedom that appear most naturally in the pNRQCD part of the matching are singlet and octet fields.

(c) As a consequence of (b), only one time appears in the computation.

(d) The gluon fields appear in the NRQCD part of the matching procedure in terms of Wilson-loop amplitudes. Therefore the formulation will be explicitly gauge invariant at each step.

(e) Gauge-invariant expressions can be obtained for the potentials that encode all the corrections in $\alpha_{s}(1 / r)$ for a given order in $1 / m$ and the multipole expansion.

The results obtained within this matching procedure will be equivalent (up to field redefinitions) to those obtained in the previous section.

From points (d) and (e) above, it is clear that the Wilson-loop matching is well suited for generalization to nonperturbative cases. Therefore it provides us with a bridge between the weak-coupling matching procedure of this section and the strong-coupling one of Sec. VII.
There, the language will be exactly the one introduced here in the framework of perturbative QCD.

In the following, we define our interpolating fields, set the basis of the matching, and illustrate the procedure by discussing the static matching up to and including order $r^{2}$ in the multipole expansion. We closely follow the work of Brambilla et al. (2000), to which we refer the reader for more details of the original derivation.

\section{Interpolating fields}

Our aim is to match, in coordinate space, amplitudes defined in terms of the fields of NRQCD with amplitudes defined in terms of the fields that appear in the pNRQCD Lagrangian (60), i.e., $A_{\mu}, S$, and $O^{a}$ fields. Therefore we need to identify some interpolating fields in NRQCD that have the same quantum numbers and the same transformation properties as $S$ and $O^{a}$. The correspondence is not one to one. Given an interpolating field in NRQCD there are an infinite number of combinations of singlet and octet operators with US fields that have the same quantum numbers and therefore a nonvanishing overlap with the NRQCD operator. Fortunately, the operators in pNRQCD can be organized according to the counting of the multipole expansion. For instance, for the singlet we have

$$
\begin{aligned}
\chi^{\dagger}\left(\mathbf{x}_{2}, t\right) & \phi\left(\mathbf{x}_{2}, \mathbf{x}_{1} ; t\right) \psi\left(\mathbf{x}_{1}, t\right) \\
\rightarrow & \sqrt{Z_{s}^{(0)}(r)} S(\mathbf{r}, \mathbf{R}, t) \\
& +\sqrt{Z_{E, s}(r)} r \mathbf{r} \cdot g \mathbf{E}^{a}(\mathbf{R}, t) O^{a}(\mathbf{r}, \mathbf{R}, t)+\cdots,
\end{aligned}
$$

and for the octet

$$
\begin{aligned}
\chi^{\dagger}\left(\mathbf{x}_{2}, t\right) \phi\left(\mathbf{x}_{2}, \mathbf{R} ; t\right) T^{a} \phi\left(\mathbf{R}, \mathbf{x}_{1} ; t\right) \psi\left(\mathbf{x}_{1}, t\right) \\
\rightarrow \sqrt{Z_{o}^{(0)}(r)} O^{a}(\mathbf{r}, \mathbf{R}, t) \\
\quad+\sqrt{Z_{E, o}(r)} r \mathbf{r} \cdot g \mathbf{E}^{a}(\mathbf{R}, t) S(\mathbf{r}, \mathbf{R}, t)+\cdots,
\end{aligned}
$$

where

$$
\phi(\mathbf{y}, \mathbf{x} ; t) \equiv P \exp \left\{i \int_{0}^{1} d s(\mathbf{y}-\mathbf{x}) \cdot g \mathbf{A}(\mathbf{x}-s(\mathbf{x}-\mathbf{y}), t)\right\} .
$$

The arrows are a reminder that the two operators act on different Hilbert spaces and that the equalities hold only inside Green's functions. The factors $Z$ are normalization factors. From Eqs. (105) and (106) it follows that the operators on the left-hand side overlap at leading order in the multipole expansion with the singlet and octet fields, respectively.

The matching for the octet in Eq. (106) does not make use of a gauge-invariant operator. In a perturbative matching this is not problematic since $V_{o}$ is gauge invariant order by order in $\alpha_{s}$. However, if one aims at taking advantage of nonperturbative techniques, it is preferable to work with a manifestly gauge-invariant quantity. The simplest solution consists in replacing the $T^{a}$ color matrix on the left-hand side of Eq. (106) by a local gluonic operator $H^{a}(\mathbf{R}, t) T^{a}$ with the right transformation properties, e.g., $g \mathbf{B}^{a}(\mathbf{R}, t) T^{a}$. All $H^{a}(\mathbf{R}, t) T^{a}$ with the right 


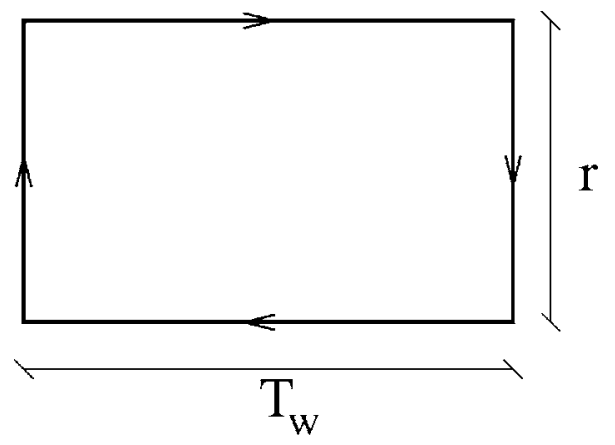

FIG. 8. A graphical representation of the static Wilson loop. We adopt the convention that the time direction is from the left to the right. Therefore the quark trajectories are represented by horizontal lines and the equal-time end-point Wilson lines by shorter vertical lines.

transformation properties will give the same potential in the weak-coupling regime corresponding to the perturbative octet potential. In the strong-coupling regime, where octet quark-antiquark fields do not exist as independent degrees of freedom, they identify different degrees of freedom and hence different potentials, corresponding to the different symmetry properties of $H^{a}$. We come back to this in full detail in Sec. VI.

\section{Matching at $\mathcal{O}\left(r^{0}, 1 / m^{0}\right)$}

In order to get $V_{s}^{(0)}$ and $Z_{s}^{(0)}$, we choose the following Green's function in NRQCD (Susskind, 1977; Brown and Weisberger, 1979):

$$
\begin{aligned}
& G_{\mathrm{NRQCD}} \\
& \quad=\left\langle\operatorname{vac}\left|\chi^{\dagger}\left(x_{2}\right) \phi\left(x_{2}, x_{1}\right) \psi\left(x_{1}\right) \psi^{\dagger}\left(y_{1}\right) \phi\left(y_{1}, y_{2}\right) \chi\left(y_{2}\right)\right| \mathrm{vac}\right\rangle \\
& \quad=\delta^{3}\left(\mathbf{x}_{1}-\mathbf{y}_{1}\right) \delta^{3}\left(\mathbf{x}_{2}-\mathbf{y}_{2}\right)\left\langle W_{\square}\right\rangle+\cdots,
\end{aligned}
$$

where the dots stand for higher-order corrections in the $1 / m$ expansion. The quantity $W_{\square}$ is the rectangular Wilson loop (Wilson, 1974) with corners $x_{1}=\left(T_{W} / 2, \mathbf{r} / 2\right)$, $x_{2}=\left(T_{W} / 2,-\mathbf{r} / 2\right), \quad y_{1}=\left(-T_{W} / 2, \mathbf{r} / 2\right), \quad$ and $\quad y_{2}$ $=\left(-T_{W} / 2,-\mathbf{r} / 2\right)$ :

$$
W_{\square} \equiv P \exp \left\{-i g \oint_{r \times T_{W}} d z^{\mu} A_{\mu}(z)\right\} .
$$

A graphical representation is given in Fig. 8. We also define

$$
\langle\cdots\rangle \equiv\langle\operatorname{vac}|\operatorname{Tr}\{\cdots\}| \operatorname{vac}\rangle=\int \mathcal{D} A \mathcal{D} q \mathcal{D} \bar{q} e^{-i S^{(0)}} \operatorname{Tr}\{\cdots\},
$$

where $S^{(0)}$ is the pure Yang-Mills plus light-quark action of QCD and the path integral is over all light fields.

Equation (105) states that the leading overlap of the Green's function (108) is with the singlet propagator in pNRQCD. Indeed, in pNRQCD we get in the static limit and at the zeroth order in the multipole expansion:

$$
G_{\mathrm{pNRQCD}}=Z_{s}^{(0)}(r) \delta^{3}\left(\mathbf{x}_{1}-\mathbf{y}_{1}\right) \delta^{3}\left(\mathbf{x}_{2}-\mathbf{y}_{2}\right) e^{-i T_{W} V_{s}^{(0)}(r)}
$$

In order to single out the soft scale, we consider the large $T_{W}$ limit of the Wilson loop (equivalent to setting $E \rightarrow 0)$ :

$$
\frac{i}{T_{W}} \ln \left\langle W_{\square}\right\rangle=u_{0}(r)+i \frac{u_{1}(r)}{T_{W}}+\mathcal{O}\left(\frac{1}{T_{W}^{2}}\right) \text { for } T_{W} \rightarrow \infty,
$$

then from the matching condition $G_{\mathrm{NRQCD}}=G_{\mathrm{pNRQCD}}$ we obtain

$$
\begin{aligned}
& V_{s}^{(0)}(r) \equiv-C_{F} \frac{\alpha_{V_{s}}(r)}{r}=u_{0}(r), \\
& \ln Z_{s}^{(0)}(r)=u_{1}(r) .
\end{aligned}
$$

The matching does not rely on any perturbative expansion in $\alpha_{s}$. However, since we are concerned with the weak-coupling situation, the quantities on the right-hand side of Eqs. (113) and (114) can be evaluated expanding order by order in $\alpha_{s}$. At LO in $\alpha_{s}$ we have

$$
V_{s}^{(0)}(r)=-C_{F} \frac{\alpha_{s}}{r} \quad \text { or } \alpha_{V_{s}}=\alpha_{s}
$$

$$
Z_{s}^{(0)}(r)=N_{c} \text {. }
$$

In order to get $V_{o}^{(0)}$ and $Z_{o}^{(0)}$ one proceeds in a similar way. We choose the NRQCD Green's function:

$$
\begin{aligned}
G_{\mathrm{NRQCD}}^{a b} & \\
= & \langle\operatorname{vac}| \chi^{\dagger}\left(x_{2}\right) \phi\left(\mathbf{x}_{2}, \frac{\mathbf{x}_{1}+\mathbf{x}_{2}}{2} ; \frac{T_{W}}{2}\right) T^{a} \\
& \times \phi\left(\frac{\mathbf{x}_{1}+\mathbf{x}_{2}}{2}, \mathbf{x}_{1} ; \frac{T_{W}}{2}\right) \psi\left(x_{1}\right) \psi^{\dagger}\left(y_{1}\right) \\
& \times \phi\left(\mathbf{y}_{1}, \frac{\mathbf{y}_{1}+\mathbf{y}_{2}}{2} ;-\frac{T_{W}}{2}\right) T^{b} \phi\left(\frac{\mathbf{y}_{1}+\mathbf{y}_{2}}{2}, \mathbf{y}_{2} ;-\frac{T_{W}}{2}\right) \\
& \times \chi\left(y_{2}\right)|\mathrm{vac}\rangle \\
= & \delta^{3}\left(\mathbf{x}_{1}-\mathbf{y}_{1}\right) \delta^{3}\left(\mathbf{x}_{2}-\mathbf{y}_{2}\right)\left\langle T^{a} W_{\square} T^{b}\right\rangle+\cdots,
\end{aligned}
$$

where in the last line the color matrices are understood as inserted in the static Wilson loop at the points $\left(\mathbf{R}, T_{W} / 2\right)$ and $\left(\mathbf{R},-T_{W} / 2\right)$. The dots stand for higherorder corrections in the $1 / \mathrm{m}$ expansion.

Equation (106) states that the leading overlap of the Green's function (117) is with the octet propagator in pNRQCD. Indeed, in pNRQCD we obtain in the static limit and at zeroth order in the multipole expansion

$$
\begin{aligned}
G_{\mathrm{pNRQCD}}^{a b}= & Z_{o}^{(0)}(r) \delta^{3}\left(\mathbf{x}_{1}-\mathbf{y}_{1}\right) \delta^{3}\left(\mathbf{x}_{2}-\mathbf{y}_{2}\right) e^{-i T_{W} V_{o}^{(0)}(r)} \\
& \times\left\langle\phi_{a b}^{\mathrm{adj}}\left(T_{W} / 2,-T_{W} / 2\right)\right\rangle,
\end{aligned}
$$

where the Wilson line, 


$$
\begin{aligned}
\phi\left(T_{W} / 2,-T_{W} / 2\right) & \equiv \phi\left(T_{W} / 2, \mathbf{R},-T_{W} / 2, \mathbf{R}\right) \\
& =P \exp \left\{-i g \int_{-T_{W} / 2}^{T_{W} / 2} d t A_{0}(\mathbf{R}, t)\right\},
\end{aligned}
$$

is evaluated in the adjoint representation. As in the singlet case, we define

$$
\begin{aligned}
\frac{i}{T_{W}} & \ln \frac{\left\langle T^{a} W_{\square} T^{b}\right\rangle}{\left\langle\phi_{a b}^{\mathrm{adj}}\left(T_{W} / 2,-T_{W} / 2\right)\right\rangle} \\
& =v_{0}(r)+i \frac{v_{1}(r)}{T_{W}}+\mathcal{O}\left(\frac{1}{T_{W}^{2}}\right) \text { for } T_{W} \rightarrow \infty .
\end{aligned}
$$

From the matching condition $G_{\mathrm{NRQCD}}^{a b}=G_{\mathrm{pNRQCD}}^{a b}$ we obtain

$$
\begin{aligned}
& V_{o}^{(0)}(r) \equiv\left(\frac{C_{A}}{2}-C_{F}\right) \frac{\alpha_{V_{o}}(r)}{r}=v_{0}(r), \\
& \ln Z_{o}^{(0)}(r)=v_{1}(r) .
\end{aligned}
$$

Again, the formulas above do not rely on any expansion in $\alpha_{s}$. However, in the weak-coupling situation, the quantities on the right-hand sides of Eqs. (120) and (121) can be expanded order by order in $\alpha_{s}$. At LO in $\alpha_{s}$ we obtain

$$
\begin{aligned}
& V_{o}^{(0)}(r)=\left(\frac{C_{A}}{2}-C_{F}\right) \frac{\alpha_{s}}{r} \text { or } \alpha_{V_{o}}=\alpha_{s}, \\
& Z_{o}^{(0)}(r)=T_{F} .
\end{aligned}
$$

Note that, despite the octet matching procedure being gauge dependent, the octet static potential obtained in this way is not at any finite order in perturbation theory (it corresponds to the pole of the octet static propagator). All the gauge dependence goes into the normalization factor $Z_{o}^{(0)}$. In this respect, it is worthwhile to observe that the string $\left\langle\phi_{a b}^{\mathrm{adj}}\left(T_{W} / 2,-T_{W} / 2\right)\right\rangle$ does not give contributions to the potential at any finite order in perturbation theory, but it does to $Z_{o}^{(0)}$.

\section{Matching at $\mathcal{O}\left(r^{1}, 1 / m^{0}\right)$ and $\mathcal{O}\left(r^{2}, 1 / m^{0}\right)$}

At $\mathcal{O}(r)$, there are no additional contributions to the singlet and octet matching potentials and to the normalization factors. At this order in the multipole expansion one finds $V_{A}$ and $V_{B}$. In the weak-coupling regime at LO in $\alpha_{s}$ they are

$$
V_{A}(r)=1, \quad V_{B}(r)=1 .
$$

At $\mathcal{O}\left(r^{2}\right)$, one finds the next-to-leading contributions to the singlet and octet static potentials and to the singlet static normalization factor.

The NLO correction in the multipole expansion to the singlet static propagator (111) is given by (see Fig. 9)

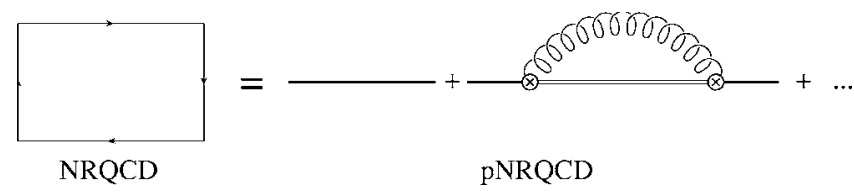

FIG. 9. The matching of $V_{s}^{(0)}$ and $Z_{s}^{(0)}$ at next-to-leading order (NLO) in the multipole expansion. On the left-hand side is the Wilson loop in NRQCD, on the right-hand side are the pNRQCD propagators. The first and second diagrams on the right-hand side symbolically represent the first and second terms in Eq. (125).

$$
\begin{aligned}
G_{\mathrm{PNRQCD}}= & Z_{s}^{(0)}(r) \delta^{3}\left(\mathbf{x}_{1}-\mathbf{y}_{1}\right) \delta^{3}\left(\mathbf{x}_{2}-\mathbf{y}_{2}\right) e^{-i T_{W} V_{s}^{(0)}(r)} \\
& \times\left(1-\frac{T_{F}}{N_{c}} V_{A}^{2}(r) \int_{-T_{W} / 2}^{T_{W} / 2} d t \int_{-T_{W} / 2}^{t} d t^{\prime}\right. \\
& \times e^{-i\left(t-t^{\prime}\right)\left(V_{o}^{(0)}-V_{s}^{(0)}\right)} \\
& \left.\times\left\langle\mathbf{r} \cdot g \mathbf{E}^{a}(t) \phi_{a b}^{\mathrm{adj}}\left(t, t^{\prime}\right) \mathbf{r} \cdot g \mathbf{E}^{b}\left(t^{\prime}\right)\right\rangle\right),
\end{aligned}
$$

where fields with only temporal arguments are evaluated in the center-of-mass coordinate. From the matching condition $G_{\mathrm{NRQCD}}=G_{\mathrm{pNRQCD}}$, we obtain $Z_{s}^{(0)}$ and $V_{s}^{(0)}$ at NLO in the multipole expansion:

$$
\begin{aligned}
V_{s}^{(0)}(r)= & u_{0}(r)+\frac{T_{F}}{N_{c}} V_{A}^{2}(r) \lim _{T_{W} \rightarrow \infty} \frac{i}{T_{W}} \int_{-T_{W^{\prime}}}^{T_{W} / 2} d t \\
& \times \int_{-T_{W^{\prime}} / t}^{t} d t^{\prime} e^{-i\left(t-t^{\prime}\right)\left(V_{o}^{(0)}-V_{s}^{(0)}\right)} \\
& \times\left\langle\mathbf{r} \cdot g \mathbf{E}^{a}(t) \phi_{a b}^{\mathrm{adj}}\left(t, t^{\prime}\right) \mathbf{r} \cdot g \mathbf{E}^{b}\left(t^{\prime}\right)\right\rangle, \\
\ln Z_{s}^{(0)}(r)= & u_{1}(r)+\frac{T_{F}}{N_{c}} V_{A}^{2}(r) \int_{-\infty}^{\infty} d t \\
& \times \int_{-\infty}^{t} d t^{\prime} e^{-i\left(t-t^{\prime}\right)\left(V_{o}^{(0)}-V_{s}^{(0)}\right)} \\
& \times\left\langle\mathbf{r} \cdot g \mathbf{E}^{a}(t) \phi_{a b}^{\mathrm{adj}}\left(t, t^{\prime}\right) \mathbf{r} \cdot g \mathbf{E}^{b}\left(t^{\prime}\right)\right\rangle .
\end{aligned}
$$

Equations (126) and (127) do not rely on any perturbative expansion in $\alpha_{s}$. However, since we are considering the weak-coupling case, they can be evaluated order by order in $\alpha_{s}$ and one can obtain the leading logarithmic contribution to the static potential. This comes from the three-loop IR logarithmic divergence of the Wilson loop first noticed by Appelquist et al. (1978) (see also Kummer et al., 1996). The calculation may be done in various ways depending on how divergences are regularized. Obviously the scheme adopted for calculating the Wilson loop must be the same as that adopted for calculating the loop diagram in $\mathrm{pNRQCD}$. This study has been performed by Brambilla et al. (1999b, 2000) giving 


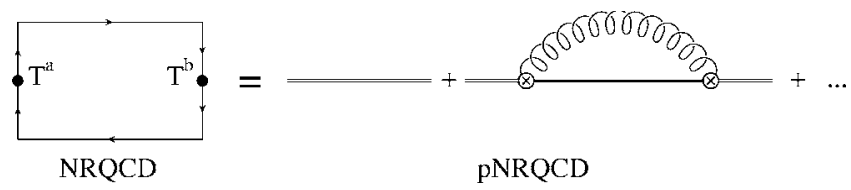

FIG. 10. The matching of $V_{o}^{(0)}$ and $Z_{o}^{(0)}$ at NLO in the multipole expansion. On the left-hand side is the Wilson loop in NRQCD with color matrix insertions, on the right-hand side are the pNRQCD propagators.

$$
\begin{aligned}
& V_{s}^{(0)}\left(r, \nu_{u s}\right)=-C_{F} \frac{\alpha_{V_{s}}(r)}{r}=\left(u_{0}(r)\right)_{\text {two-loop }} \\
&-\frac{C_{F} C_{A}^{3}}{12} \frac{\alpha_{s}}{r} \frac{\alpha_{s}^{3}}{\pi} \ln \left(r \nu_{u s}\right), \\
& \ln Z_{s}^{(0)}\left(r, \nu_{u s}\right)=\left(u_{1}(r)\right)_{\text {two-loop }}+\frac{C_{F} C_{A}^{2}}{2} \frac{\alpha_{s}^{3}}{\pi} \ln \left(r \nu_{u s}\right) .
\end{aligned}
$$

The two-loop expression for $u_{0}(r)$ is given by $-C_{F} \alpha_{V_{s}}(r)_{\text {two-loop }} / r$ and the two-loop expression for $\alpha_{V}$ can be found in Eq. (101). The contributions proportional to $\ln \left(r \nu_{u s}\right)$ in Eqs. (128) and (129) would be zero in QED. The fact that $\alpha_{V_{s}}$ depends on the IR behavior of the theory is therefore a distinct feature of QCD, more specifically, of the non-Abelian nature of QCD, which allows gluons to interact with themselves at arbitrarily small energy scales. We stress that in order to match the normalization factor (129), it is necessary to take into account contributions coming from the end-point Wilson lines, which can be considered irrelevant only at order $\left(1 / T_{W}\right)^{0}$, i.e., for the potential (note that this does not require any special assumption about the large-time behavior of the gluon fields).

The NLO correction to Eq. (118) in the multipole expansion comes from the graph shown in Fig. 10. We omit a term proportional to $V_{B}^{2}$ of the type shown in Fig. 11 and terms which contain operators like $\operatorname{Tr}\left\{\mathbf{r}^{i} \mathbf{r}^{j}\left[\mathbf{D}^{i}, \mathbf{E}^{j}\right] O O^{\dagger}\right\}$, because in perturbation theory they neither contribute to the octet matching potential nor to the normalization. The reason is that in contrast to the nonperturbative regime where we may have dependencies on the scale $\Lambda_{\mathrm{QCD}}$, in perturbation theory loops on octet lines are scaleless and vanish in DR. With a calculation analogous to that in the singlet case we obtain at leading logarithmic three-loop accuracy

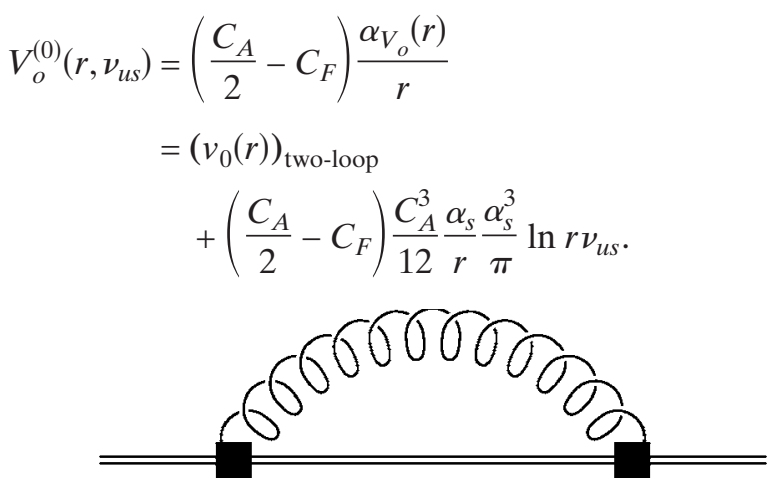

FIG. 11. Octet self-energy graph proportional to $V_{B}^{2}$.
The two-loop expression for $v_{0}(r)$ is given by $\left(C_{A}\right.$ $\left.-C_{F} / 2\right) \alpha_{V_{o}}(r)_{\text {two-loop }} / r$ and for the two-loop expression of $\alpha_{V_{o}}$, see Eq. (103). Similarly $Z_{o}^{(0)}$ may also be calculated, but only in a specific gauge.

\section{Matching at order $r^{0}\left(1 / m, 1 / m^{2}\right.$, and beyond $)$}

Following this method, one may consider $1 / \mathrm{m}$ corrections. If one works at LO in the multipole expansion, the singlet and octet fields decouple. If we further focus on the singlet sector, the computations would be similar to those that appear in Sec. VII.E.4 for the strong-coupling regime. This is the case because we are actually performing the matching order by order in $1 / \mathrm{m}$ and to any order in $\alpha_{s}$. Therefore the expressions obtained in the strong-coupling regime also hold here up to corrections due to US effects. This reasoning also applies to what in Sec. VII is called the "quantum-mechanical matching" (see Sec. VII.E), where explicit expressions in terms of Wilson-loop amplitudes for the real and imaginary parts of the pNRQCD potentials are derived. Those expressions are also valid in the perturbative regime, if they are understood to be at $\mathcal{O}\left(r^{0}\right)$ in the multipole expansion. Note that the Wilson loops multiplying delta functions of $\mathbf{r}$ or derivatives of them are zero in the perturbative regime since they become dimensionless objects and vanish in DR. In particular, this applies to the gluonic correlators that appear in the imaginary part of the potential. Finally, we note that nonanalytic terms due to the scale $\sqrt{m \Lambda_{\mathrm{QCD}}}$ do not appear here since for $\Lambda_{\mathrm{QCD}}$ $\lesssim E$, this three-momentum scale has not been integrated out.

\section{G. Observables: spectrum and inclusive decay widths}

We have finally built the pNRQCD Lagrangian and are in the position to calculate observables with it. We consider observables (being the theoretically cleanest ones) that only involve the calculation of the NR propagator (Green's function) of the system projected onto the colorless sector of a quark-antiquark pair (with $P_{s}$ the corresponding projector) and the gluonic vacuum,

$$
\begin{aligned}
& \Pi\left(E, \mathbf{r}, \mathbf{r}^{\prime}\right) \equiv i \int d t d^{3} \mathbf{R} e^{i E t}\left\langle\operatorname{vac}\left|T\left\{S\left(\mathbf{r}^{\prime}, 0,0\right) S^{\dagger}(\mathbf{r}, \mathbf{R}, t)\right\}\right| \operatorname{vac}\right\rangle \\
& =\left\langle\mathbf{r}^{\prime}\left|G_{s}(E)\right| \mathbf{r}\right\rangle, \\
& G_{s}(E) \equiv P_{s}\left\langle\operatorname{vac}\left|\frac{1}{H-E}\right| \operatorname{vac}\right\rangle P_{s}=G_{c}(E)+\delta G_{s},
\end{aligned}
$$

where $H$ is the pNRQCD Hamiltonian, $G_{c}$ the Coulomb Green's function defined in Fig. 5, and $E$ the energy measured from the threshold $2 \mathrm{~m}$.

Besides the heavy-quarkonium spectrum (i.e., the poles of the Green's function), we consider inclusive (electromagnetic) decay widths, NR sum rules, and $t-\bar{t}$ 
production near threshold. For these the normalization at the origin will be important, ${ }^{12}$ i.e., the object $\langle\mathbf{r}$ $\left.=0\left|G_{s}(E)\right| \mathbf{r}=0\right\rangle$ has to be computed.

In pNRQCD, there are only potential and US loops. Within pNRQCD, talking about potential loops is nothing but talking about quantum-mechanical perturbation theory:

$$
\delta G_{s}^{\text {pot. }} \longrightarrow_{\delta V_{s}}+\cdots \sim G_{c} \delta V_{s} G_{c}+\cdots,
$$

where the black square represents a generic $\delta V_{s}$ correction to the singlet Coulomb Hamiltonian.

US loops can be computed using standard Feynmandiagram techniques, where it is sometimes convenient to work in momentum space for the US momenta and in position space for the soft scale [this is certainly so if one wants to do standard (finite) quantum-mechanical perturbation theory, although it is clearly possible to do it in momentum space]. We illustrate the procedure with the first US contribution to $G_{s}$ :

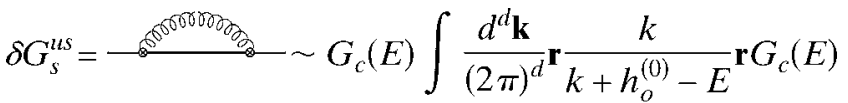

$$
\begin{aligned}
& \sim G_{c}(E) \mathbf{r}\left(h_{o}^{(0)}-E\right)^{3}\left\{\frac{1}{\epsilon}+\gamma+\ln \frac{\left(h_{o}^{(0)}-E\right)^{2}}{\nu_{u s}^{2}}\right. \\
& +C\} \mathbf{r} G_{c}(E) \text {, }
\end{aligned}
$$

where $d=3+2 \epsilon$. We can see that the result is UV divergent. This is not a problem in an EFT in which such divergences can (and should) be absorbed in the matching coefficients of the EFT, i.e., in the potentials. Moreover, there are other sources of logarithmic UV divergences, proportional to $\ln \nu_{p}$, coming from potential loops. They show up either by going to high enough orders in quantum-mechanical perturbation theory [for instance, if we are interested in computing the spectrum at $\left.\mathcal{O}\left(m \alpha_{s}^{6}\right)\right]$,

$$
G_{c}(E) \delta V_{s} G_{c}(E) \cdots \delta V_{s} G_{c}(E),
$$

or by inserting sufficiently singular operators in the computation (as is the case for the renormalization of the matching coefficient of the electromagnetic current). These divergences can be absorbed in the matching coefficients of the local potentials [those proportional to $\delta^{(3)}(\mathbf{r})$ or its derivatives] or in the matching coefficients associated with the currents. Let us explain in detail how this works. Since the singular behavior of the potential loops appears for $|\mathbf{p}| \gg \alpha_{s} / r$, a perturbative expansion in

\footnotetext{
${ }^{12}$ Other observables that do not belong to this category are semi-inclusive radiative decay widths, which have been studied by Garcia i Tormo and Soto (2004) and are considered in Sec. VIII.G, or heavy-quarkonium production, for which an analysis in the weak-coupling regime is available (Beneke et al., 2000).
}
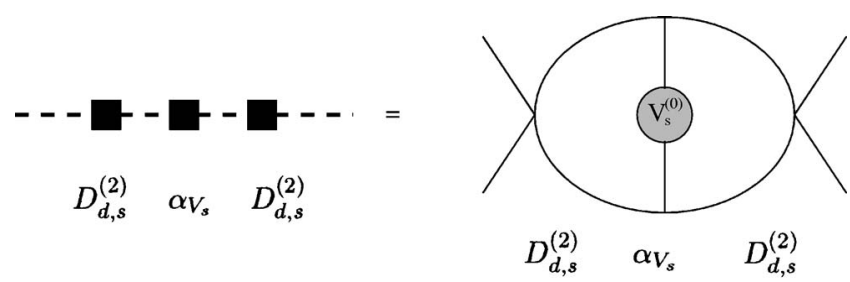

FIG. 12. One possible contribution to the running of $D_{d, s}^{(2)}$ at next-to-leading-logarithm order (NLL). The first picture represents the calculation in terms of the free quark-antiquark propagator $G_{c}^{(0)}$ and the potentials (the small rectangles). The picture on the right is the representation within a more standard diagrammatic interpretation in terms of quarks and antiquarks. The delta potentials are displayed as local interactions and the Coulomb potential as an extended object in space (but not in time).

$\alpha_{s}$ is allowed in $G_{c}(E)$, which can be approximated by the free propagator:

$$
-\cdots G_{c}^{(0)}(E)=\frac{1}{E-\mathbf{p}^{2} / m} .
$$

Therefore a practical simplification follows from the fact that the Coulomb potential $-C_{F} \alpha_{s} / r$ can be considered a perturbation as far as the computation of the $\ln \nu_{p} \mathrm{UV}$ divergences is concerned. Moreover, each $G_{c}^{(0)}$ produces a potential loop and one extra power of $m$ in the numerator, which eliminates the powers of $1 / \mathrm{m}$ in the different potentials. This allows the mixing of potentials with different powers of $1 / \mathrm{m}$. One typical example is the diagram in Fig. 12, which corresponds to

$$
\begin{aligned}
& G_{c}^{(0)}(E) \frac{\pi C_{F} D_{d, s}^{(2)}}{m^{2}} \delta^{(3)}(\mathbf{r}) G_{c}^{(0)}(E) C_{F} \frac{\alpha_{V_{s}}}{r} G_{c}^{(0)}(E) \\
& \times \frac{\pi C_{F} D_{d, s}^{(2)}}{m^{2}} \delta^{(3)}(\mathbf{r}) G_{c}^{(0)}(E) .
\end{aligned}
$$

The relevant computation gives

$$
\begin{aligned}
\langle\mathbf{r}= & \left.0\left|G_{c}^{(0)}(E) C_{F} \frac{\alpha_{V_{s}}}{r} G_{c}^{(0)}(E)\right| \mathbf{r}=0\right\rangle \\
& \sim \int \frac{d^{d} p^{\prime}}{(2 \pi)^{d}} \int \frac{d^{d} p}{(2 \pi)^{d}} \frac{m}{\mathbf{p}^{\prime 2}-m E} C_{F} \frac{4 \pi \alpha_{V_{s}}}{\mathbf{q}^{2}} \frac{m}{\mathbf{p}^{2}-m E} \\
& \sim-C_{F} \frac{m^{2} \alpha_{V_{s}}}{16 \pi} \frac{1}{\epsilon},
\end{aligned}
$$

where $\mathbf{q}=\mathbf{p}-\mathbf{p}^{\prime}$. This divergence can be absorbed in $D_{d, s}^{(2)}$ contributing to its running as follows:

$$
\nu_{p} \frac{d}{d \nu_{p}} D_{d, s}^{(2)}\left(\nu_{p}\right) \sim \alpha_{V_{s}}\left(\nu_{p}\right) D_{d, s}^{(2) 2}\left(\nu_{p}\right)+\cdots .
$$

It is particularly appealing how the EFT framework gives a solution to the problem of the UV divergences one finds in standard quantum-mechanical perturbationtheory calculations. When potential divergences are found it can be more convenient to work in a momen- 
tum representation [see, for instance, Czarnecki et al. (1999b)]. Nevertheless, it is also possible to handle the UV divergences in position space (Yelkhovsky, 2001). Either way, the computation should be performed in the same scheme used to compute the potentials (see Sec. IV.E for details).

\section{Heavy-quarkonium mass}

After this discussion and taking into account the power-counting rules given in Sec. IV.B, one can obtain the different observables up to some order in $v \sim \alpha_{s}$. For instance, the level of precision of the perturbative computation for the heavy-quarkonium mass,

$$
M_{n l j}^{\text {pert. }}=2 m+\sum_{m=2}^{\infty} A_{n l j}^{(m)} \alpha_{s}^{m},
$$

is as follows (some results were actually computed prior to the existence of pNRQCD). The $\mathcal{O}\left(m \alpha_{s}^{2}\right)$ result is nothing but the positroniumlike result with the proper color factor. The $\mathcal{O}\left(m \alpha_{s}^{3}\right)$ contribution was computed by Billoire (1980). The $\mathcal{O}\left(m \alpha_{s}^{4}\right)$ term was computed by Melnikov and Yelkhovsky (1998), Pineda and Yndurain (1998, 2000), Penin and Pivovarov (1999); the one at $\mathcal{O}\left(m \alpha_{s}^{5} \ln \alpha_{s}\right)$ by Brambilla et al. (1999a), Kniehl and Penin (2000c), Hoang, Manohar, and Stewart (2001); the next-to-next-to-next-to-leading-order (NNNLO) large$\beta_{0}$ result by Hoang (2000), Kiyo and Sumino (2000); and the computations that complete the NNNLO result for the ground state (but without the static-potential threeloop coefficient) by Kniehl et al. (2002a), Penin and Steinhauser, (2002). ${ }^{13}$ Logarithms have also been resummed for the heavy-quarkonium mass (see Sec. IV.H for details).

In principle, for the bottomonium ground state, finite charm-mass effects have to be taken into account since the soft scale is of the order of the charm mass. They can be found in the articles by Eiras and Soto (2000); Hoang (2000); Melles (2000); Wang and Yao (2004).

So far, nonperturbative effects have not been discussed. Therefore it was implicitly assumed that $\Lambda_{\mathrm{QCD}}$ $\ll m v^{2}$, which makes them relevant at $\mathcal{O}\left(m \alpha_{s}^{5}\right)$ where US modes appear for the first time. This assumption may be reasonable for $t-\bar{t}$ systems, but for bottomonium and charmonium it is more questionable. In the situation $\Lambda_{\mathrm{QCD}} \simeq m v^{2}$, one cannot compute using perturbation theory at the US scale. In this situation (which may be relevant for bottomonium), the energy of the heavy quarkonium reads as follows:

\footnotetext{
${ }^{13}$ The application of pNRQED (the QED version of pNRQCD) and, in general, of factorization with DR, has also led to a plethora of results for the spectra of positronium (Czarnecki et al., 1999a; Melnikov and Yelkhovsky, 1999b, 2001; Pineda and Soto, 1999; Kniehl and Penin, 2000b).
}

$$
M_{n l j}=2 m+\sum_{m=2}^{\infty} A_{n l j}^{(m)}\left(\nu_{u s}\right) \alpha_{s}^{m}+\delta M_{n l j}^{\mathrm{US}}\left(\nu_{u s}\right),
$$

where the $\nu_{u s}$ scale dependence of the different pieces cancels in the overall sum (for the perturbative sum, this dependence first appears in $\left.A_{n l j}^{(5)}\right)$ and $\left(E_{n} \equiv A_{n l j}^{(2)} \alpha_{s}^{2}\right)$

$$
\begin{aligned}
\delta M_{n l j}^{\mathrm{US}}\left(\nu_{u s}\right) \simeq & \delta M_{n l}^{\mathrm{US}}\left(\nu_{u s}\right) \\
= & \frac{T_{F}}{3 N_{c}} \int_{0}^{\infty} d t\left\langle n, l\left|\mathbf{r} e^{-t\left(h_{o}^{(0)}-E_{n}\right)} \mathbf{r}\right| n, l\right\rangle \\
& \times\left\langle g \mathbf{E}^{a}(t) \phi(t, 0)_{a b}^{\mathrm{adj}} g \mathbf{E}^{b}(0)\right\rangle\left(\nu_{u s}\right),
\end{aligned}
$$

for which one can think of several possibilities depending on the relative size between $m v^{2}$ and $\Lambda_{\mathrm{QCD}}$. In the limit $m v^{2} \gg \Lambda_{\mathrm{QCD}}$, the result obtained by Penin and Steinhauser (2002) is the combination

$$
A_{n l j}^{(5)}\left(\nu_{u s}\right) \alpha_{s}^{5}+\left.\delta M_{n l j}^{\mathrm{US}}\left(\nu_{u s}\right)\right|_{\mathcal{O}\left(\alpha_{s}^{5}\right) \text { pert. }} .
$$

The expression for the nonperturbative object looks similar to Eq. (140) but with an UV cutoff $\Lambda$ such that $m v^{2} \gg \Lambda \gg \Lambda_{\mathrm{QCD}}$. Therefore we have

$$
\delta M_{n l j}^{\mathrm{US}}\left(\nu_{u s}\right)=\delta M_{n l j}^{\text {pert.,US }}\left(\nu_{u s} ; \Lambda\right)+\delta M_{n l j}^{\mathrm{US}}(\Lambda) .
$$

The study of the nonperturbative effects in this limit, often called the Voloshin-Leutwyler limit, has a long history starting from Voloshin (1979) and Leutwyler (1981). $\delta M_{n l j}^{\mathrm{US}}(\Lambda)$ reads [this expression follows by Fourier transforming to energy space Eq. (140) and setting $\nu_{u s}=\Lambda$ ]

$$
\begin{aligned}
\delta M_{n l j}^{\mathrm{US}}(\Lambda)= & \frac{g^{2}}{6 N_{c}}\langle\operatorname{vac}| E_{j}^{a}(0) \\
& \times\left\langle n, l\left|\mathbf{r}\left[\frac{1}{E_{n}-h_{o}^{(0)}-i D_{0}^{\mathrm{adj}}}\right]_{a b} \mathbf{r}\right| n, l\right\rangle \\
& \times E_{j}^{b}(0)|\mathrm{vac}\rangle .
\end{aligned}
$$

A notation closer to the one used by Voloshin (1979) can be obtained by going to a Hamiltonian formulation (for instance, fixing the gauge $A_{0}=0$ ). This corresponds to replacing $i D_{0}^{\text {adj }}$ by $H^{(0)}$, where $H^{(0)}$ is defined in Eq. (196) and the physical states are constrained to satisfy the Gauss law (projected to the octet sector),

$$
\begin{aligned}
\left.\mathbf{D} \cdot \boldsymbol{\Pi}^{a} \mid \text { phys }\right\rangle= & \left(\int d^{3} R \operatorname{Tr}\left\{O^{\dagger}\left[g T^{a}, O\right]\right\}\right. \\
& \left.\left.+\bar{q} \gamma^{0} T^{a} q\right) \mid \text { phys }\right\rangle,
\end{aligned}
$$

where $\boldsymbol{\Pi}^{a}$ is the canonical momentum conjugated to $\mathbf{A}^{a}$. As long as we do not study the fine and hyperfine splittings (see Leutwyler, 1981; Curci et al., 1983; Campostrini et al., 1986; Krämer et al., 1992; Titard and Yndurain, 1995; Pimeda, 1997a, for such studies in the Voloshin-Leutwyler limit), the corrections do not depend on $j$ (total angular momentum) and $s$ (spin) so we shall not display these indices in the states. The octet propagator mixes low $\mathcal{O}\left(i D_{0}^{\text {adj }} \sim \Lambda_{\mathrm{QCD}}\right)$ and high energies $\mathcal{O}\left(h_{o}^{(0)} \sim E_{n} \sim m v^{2}\right)$. Therefore an operator product ex- 
pansion can be performed whose expansion parameter is of order

$$
\left(\frac{i D_{0}^{\mathrm{adj}}}{E_{n}-h_{o}}\right)^{2} \sim\left(\frac{\Lambda_{\mathrm{QCD}}}{m \beta_{n}^{2}}\right)^{2}
$$

and one obtains

$$
\delta M_{n l}^{\mathrm{US}}(\Lambda)=\sum_{r=0}^{\infty} C_{r} O_{r} \equiv \sum_{r=0}^{\infty} \delta E_{n l}^{(r)},
$$

where

$$
\begin{aligned}
C_{r}= & \left\langle n, l\left|\mathbf{r}\left(\frac{1}{E_{n}-h_{o}}\right)^{2 r+1} \mathbf{r}\right| n, l\right\rangle, \\
O_{r}= & \frac{g^{2}}{54}\langle\operatorname{vac}| \operatorname{Tr}\left(\left[D_{0}(0),\left[\cdots\left[D_{0}(0), \mathbf{E}(0)\right] \cdots\right]\right.\right. \\
& \times\left[D_{0}(0),\left[\cdots\left[D_{0}(0), \mathbf{E}(0)\right] \cdots\right]\right)|\operatorname{vac}\rangle,
\end{aligned}
$$

and the trace is in the adjoint representation. $\delta E_{n l}^{(0)}$ has been obtained by Leutwyler (1981); Voloshin (1982); Pineda (1997b) and $\delta E_{n l}^{(1)}$ by Pineda (1997b). For further details, we refer the reader to these works.

What we have discussed applies for $t \bar{t}$ production near threshold. In the case of bottomonium or charmonium, it is more likely that the kinematical situations $m v^{2}$ $\sim \Lambda_{\mathrm{QCD}}$ (in which the whole functional form of the chromoelectric correlator is needed) or $\Lambda_{\mathrm{QCD}} \gg m v^{2}$ apply. This last situation is discussed in Sec. VII. A phenomenological analysis is presented in Sec. VIII.A.

\section{Inclusive decay widths}

It is rather easy, after the matching has been performed, to calculate in pNRQCD the inclusive decay width of a heavy quarkonium $H$ into light particles. This is the imaginary part of the singlet propagator pole in the complex plane and may be calculated as (at LO in $\operatorname{Im} H$ )

$\Gamma(H \rightarrow$ light particles $)=-2\langle n, l, s, j|\operatorname{Im} H| n, l, s, j\rangle$.

The imaginary part of the pNRQCD Hamiltonian has been written in Eqs. (75) and (76). It depends on delta (or derivatives of delta) potentials and does not mix singlet and octet fields. The states $|n, l, s, j\rangle$ are the eigenstates of the pNRQCD Hamiltonian. For electromagnetic inclusive decays, $\operatorname{Im} f_{\mathrm{EM}}^{\mathrm{pNR}}\left({ }^{3} S_{1}\right)$ is needed (or equivalently the matching coefficient of the electromagnetic current, $\left.b_{1, \mathrm{pNR}}^{v}\right)$ for the decay into $e^{+} e^{-}$and $\operatorname{Im} f_{\mathrm{EM}}^{\mathrm{NR}}\left({ }^{1} S_{0}\right)$, for the decay into $\gamma \gamma$. The first matching coefficient is known at present with two-loop accuracy (Källen and Sarby, 1955; Beneke et al., 1998; Czarnecki and Melnikov, 1998) in a closed analytic form. For the second, besides the one-loop result by Harris and Brown (1957), a semianalytic two-loop result was obtained by Czarnecki and Melnikov (2002). Apart from the electromagnetic matching coefficients, the relevant calculation is that of the residue of the NR propagator at the origin:

$$
\underset{E=E_{\text {pole }}}{\operatorname{Res}}\left\langle\mathbf{r}=0\left|G_{s}(E)\right| \mathbf{r}=0\right\rangle=\left|\phi_{n}^{(0)}\right|^{2}\left(1+\delta \phi_{n}\right)^{2},
$$

where

$$
\left|\phi_{n}^{(0)}\right|^{2}=\frac{1}{\pi}\left(\frac{m C_{F} \alpha_{s}}{2 n}\right)^{3} \equiv \rho_{n},
$$

and $E_{\text {pole }}$ is the energy for which $G_{s}(E)$ has a pole. Explicit expressions for the purely perturbative computation at NNLO can be found in the articles of Melnikov and Yelkhovsky (1999a) and Penin and Pivovarov (1999). Note that at this order the LO expressions for $\operatorname{Im} g g_{\mathrm{EM}}^{\mathrm{pNR}}\left({ }^{3} S_{1}\right)$ and $\operatorname{Im} g_{\mathrm{EM}}^{\mathrm{pNR}}\left({ }^{1} S_{0}\right)$ are also needed. Therefore, with NNLO precision, the electromagnetic decays can be written in the following way:

$$
\begin{aligned}
\Gamma\left(V_{Q}(n S) \rightarrow e^{+} e^{-}\right)= & \frac{4 C_{A}}{m^{2}} \rho_{n}\left[\operatorname{Im} f_{\mathrm{EM}}^{\mathrm{pNR}}\left({ }^{3} S_{1}\right)\left(1+\delta \phi_{n}\right)^{2}\right. \\
& \left.+\operatorname{Im} g_{\mathrm{EM}}^{\mathrm{pNR}}\left({ }^{3} S_{1}\right) \frac{E_{n}}{m}\right], \\
\Gamma\left(P_{Q}(n S) \rightarrow \gamma \gamma\right)= & \frac{4 C_{A}}{m^{2}} \rho_{n}\left[\operatorname{Im} f_{\mathrm{EM}}^{\mathrm{pNR}}\left({ }^{1} S_{0}\right)\left(1+\delta \phi_{n}\right)^{2}\right. \\
& \left.+\operatorname{Im} g_{\mathrm{EM}}^{\mathrm{pNR}}\left({ }^{1} S_{0}\right) \frac{E_{n}}{m}\right],
\end{aligned}
$$

where $V$ and $P$ stand for the vector and pseudoscalar heavy quarkonium. Some higher-order corrections are also known. The $\mathcal{O}\left(\alpha_{s}^{3} \ln \alpha_{s}\right)$ term has been computed by Kniehl et al. (2003); Hoang (2004), the $\mathcal{O}\left(\alpha_{s}^{3} \ln ^{2} \alpha_{s}\right)$ term by Kniehl and Penin (2000c). ${ }^{14}$ For RG-improved expressions, see Sec. IV.H.

For the nonperturbative corrections, a discussion similar to the mass case applies to the relative size between $\Lambda_{\mathrm{QCD}}$ and $m v^{2}$. Near the pole $E_{n}$, we have the expansion (we only consider nonperturbative corrections in what follows)

$$
\begin{aligned}
\langle\mathbf{r}= & \left.0\left|G_{s}(E)\right| \mathbf{r}=0\right\rangle \\
= & \frac{\rho_{n}+\delta \rho_{n}^{\mathrm{np}}}{E_{n}+\delta E_{n 0}^{\mathrm{np}}-E}+\mathcal{O}\left(\left(E_{n}+\delta E_{n 0}^{\mathrm{np}}-E\right)^{0}\right) \\
= & \frac{\rho_{n}}{E_{n}-E}-\frac{\rho_{n} \delta E_{n 0}^{\mathrm{np}}}{\left(E_{n}-E\right)^{2}}+\frac{\delta \rho_{n}^{\mathrm{np}}}{E_{n}-E}+\mathcal{O}\left(\left(E_{n}-E\right)^{0}\right) \\
& +\mathcal{O}\left(\delta^{\mathrm{np}} E_{n 0}^{2}\right) .
\end{aligned}
$$

On the other hand, one obtains

$$
\begin{aligned}
& \left\langle\mathbf{r}=0\left|G_{s}(E)\right| \mathbf{r}=0\right\rangle \\
& \quad \simeq\left\langle\mathbf{r}=0\left|G_{c}(E)\right| \mathbf{r}=0\right\rangle+\left\langle\mathbf{r}=0\left|\delta G_{s}^{\mathrm{np}}(E)\right| \mathbf{r}=0\right\rangle,
\end{aligned}
$$

where

\footnotetext{
${ }^{14}$ Major progress has also been made in QED for positronium decays using these techniques. See Kniehl and Penin, 2000a; Melnikov and Yelkhovsky, 2000.
} 


$$
\begin{aligned}
\langle\mathbf{r}= & 0 \mid \\
= & \frac{g^{2}}{18}\langle\operatorname{vac}| E_{j}^{a}(0)\langle\mathbf{r}=0| \frac{1}{h_{s}^{(0)}-E} \mathbf{r}\left[\frac{1}{h_{o}^{(0)}+i D_{0}^{\mathrm{adj}}-E}\right]_{a b} \mathbf{r} \\
& \times \frac{1}{h_{s}^{(0)}-E}|\mathbf{r}=0\rangle E_{j}^{b}(0)|\mathrm{vac}\rangle \\
= & -\frac{\rho_{n} \delta E_{n 0}^{\mathrm{np}}}{\left(E_{n}-E\right)^{2}}+\frac{\delta \rho_{n}^{\mathrm{np}}}{E_{n}-E}+\mathcal{O}\left(\left(E_{n}-E\right)^{0}\right) .
\end{aligned}
$$

Proceeding in the same way as before, we can factorize $m v^{2}$ from $\Lambda_{\mathrm{QCD}}$ effects:

$$
\left\langle\mathbf{r}=0\left|\delta G_{s}^{\mathrm{np}}(E)\right| \mathbf{r}=0\right\rangle=\sum_{r=0}^{\infty} C_{r}^{G} O_{r},
$$

where

$$
\begin{aligned}
C_{r}^{G} & =\left\langle\mathbf{r}=0\left|\frac{1}{h_{s}^{(0)}-E} \mathbf{r}\left(\frac{1}{h_{o}^{(0)}-E}\right)^{2 r+1} \mathbf{r} \frac{1}{h_{s}^{(0)}-E}\right| \mathbf{r}=0\right\rangle \\
& =\frac{A_{-2}^{(r)}}{\left(E_{n}-E\right)^{2}}+\frac{A_{-1}^{(r)}}{\left(E_{n}-E\right)}+\mathcal{O}\left(\left(E_{n}-E\right)^{0}\right),
\end{aligned}
$$

and $O_{r}$ is defined in Eq. (148). Now, from these expressions, we can read off the observables we are interested in, namely,

$$
\delta \rho_{n}^{\mathrm{np}} \equiv \sum_{r=0}^{\infty} \delta \rho_{n}^{(r)}=\sum_{r=0}^{\infty} A_{-1}^{(r)} O_{r}, \quad \delta E_{n 0}^{\mathrm{np}}=\frac{-1}{\rho_{n}} \sum_{r=0}^{\infty} A_{-2}^{(r)} O_{r} .
$$

This also provides a new method of obtaining the energy corrections for $l=0$ states, which can be used to check the results of the previous subsection. $\delta \rho_{n}^{(0)}$ and $\delta E_{n 0}^{(0)}$ were calculated by Voloshin (1982) and $\delta \rho_{n}^{n}$ by Pineda (1997b). We refer the reader to these works for further details.

NR sum rules and $t-\bar{t}$ production near threshold will be discussed in Secs. VIII.E and VIII.F, respectively. For those, the relevant objects to be computed are again $\langle\mathbf{r}$ $\left.=0\left|G_{s}(E)\right| \mathbf{r}=0\right\rangle$, but for arbitrary energy $E \sim m v^{2}$, and the electromagnetic matching coefficients considered before. Finally, it is also possible to obtain RG-improved expressions, which we consider in the next section.

\section{H. Renormalization group}

Schematically, we can write the pNRQCD Lagrangian as an expansion in $r$ and $1 / m$ in the following way:

$$
\mathcal{L}_{\text {pNRQCD }}=\sum_{n=-1}^{\infty} r^{n} \tilde{V}_{n} O_{n}+\frac{1}{m} \sum_{n=-2}^{\infty} r^{n} \tilde{V}_{n}^{(1)} O_{n}^{(1)}+\mathcal{O}\left(\frac{1}{m^{2}}\right),
$$

where $\tilde{V}_{n}^{(\ell)}\left(\tilde{V}_{n}^{(0)} \equiv \tilde{V}_{n}\right)$ are dimensionless constants (in four dimensions). Since they reabsorb the divergences of the EFT in the way explained in Sec. IV.G, they will depend on $\nu_{p}$ and $\nu_{u s}$. One can obtain RG-improved expressions for $\tilde{V}_{n}^{(\ell)}$ in the following way.

One first performs the matching from QCD to NRQCD. The latter depends on some matching coefficients, $c\left(\nu_{s}\right)$ and $f\left(\nu_{p}, \nu_{s}\right)$, which can be obtained order by order in $\alpha_{s}$ (with $\nu_{p}=\nu_{s}$ ) following the procedure described in Sec. II.D. In Sec. II.E, we discussed the procedure to get the running of $c$ and the soft $\left(\nu_{s}\right)$ running of $f$ at any finite order (basically using HQET techniques). Nevertheless, the running of $f\left(\nu_{p}, \nu_{s}\right)$ is more complicated beyond one loop since a dependence on $\nu_{p}$ appears. As we shall see, it can be obtained within $\mathrm{pN}$ RQCD.

The second step is the matching from NRQCD to pNRQCD. The latter depends on some matching coefficients (potentials), which typically have the following structure: $\tilde{V}\left(c\left(\nu_{s}\right), f\left(\nu_{p}, \nu_{s}\right), \nu_{s}, \nu_{u s}, r\right)$. These potentials can be obtained order by order in $\alpha_{s}$ following the procedure described in Secs. IV.E and IV.F. The integrals in the matching calculation depend on a factorization scale $\nu$, which corresponds either to $\nu_{s}$ or to $\nu_{u s}$. In an explicit calculation, they can be distinguished by looking at the UV and IR behavior of the diagrams: UV divergences are proportional to $\ln \nu_{s}$, which cancel the $\nu_{s}$ scale dependence inherited from the NRQCD matching coefficients, and IR divergences are proportional to $\nu_{u s}$. However, since we only want to perform a matching calculation at some given scale $\nu=\nu_{s}=\nu_{u s}$ (or when working order by order in $\alpha_{s}$ without attempting any resummation of logarithms), it is not necessary to distinguish between $\nu_{s}$ and $\nu_{u s}$.

The third step is to obtain the RG equations of the potentials. $\nu_{s}$ provides us with the starting point of the RG evolution with respect to $\nu_{u s}$ (up to a constant of order 1). The running with respect to $\nu_{u s}$ can then be obtained following the procedure described by Pineda and Soto (2000) and Pineda (2002b). Formally, the RG equations of the matching coefficients due to the $\nu_{u s}$ dependence read

$$
\nu_{u s} \frac{d}{d \nu_{u s}} \tilde{V}=B_{\tilde{V}}(\tilde{V}) .
$$

From a practical point of view, one can organize the RG equations within an expansion in $1 / m$ and $\alpha_{s}\left(\nu_{u s}\right)$. At $\mathcal{O}\left(1 / m^{0}\right)$, the analysis corresponds to the study of the static limit of pNRQCD, which has been carried out by Pineda and Soto (2000). Since $\tilde{V}_{-1} \neq 0$, there are relevant operators (super-renormalizable terms) in the Lagrangian and the US RG equations lose the triangular structure that they exhibited for the RG equations of $\nu_{s}$. Still, if $\tilde{V}_{-1} \ll 1$, the RG equations can be obtained as a double expansion in $\tilde{V}_{-1}$ and $\tilde{V}_{0}$, where the latter corresponds to the marginal operators (renormalizable interactions). At short distances $\left(1 / r \gg \Lambda_{\mathrm{QCD}}\right)$, this is the case for the static limit of pNRQCD. Specifically, we have $\tilde{V}_{-1}$ $=\left\{\alpha_{V_{s}}, \alpha_{V_{o}}\right\}$, which fulfills $\tilde{V}_{-1} \sim \alpha_{s}(r) \ll 1, \tilde{V}_{0}=\alpha_{s}\left(\nu_{u s}\right)$, and 
$\tilde{V}_{1}=\left\{V_{A}, V_{B}\right\} \sim 1$. Therefore we can calculate the anomalous dimensions order by order in $\alpha_{s}\left(\nu_{u s}\right)$. In addition, we also have an expansion in $\tilde{V}_{-1}$. Moreover, the specific form of the pNRQCD Lagrangian severely constrains the RG equations' general structure. Therefore, for instance, the leading nontrivial RG equation for $\alpha_{V_{s}}$ reads

$$
\begin{aligned}
\nu_{u s} \frac{d}{d \nu_{u s}} \alpha_{V_{s}}= & \frac{2}{3} \frac{\alpha_{s}}{\pi} V_{A}^{2}\left[\left(\frac{C_{A}}{2}-C_{F}\right) \alpha_{V_{o}}+C_{F} \alpha_{V_{s}}\right]^{3} \\
& +\mathcal{O}\left(\tilde{V}_{-1}^{4} \tilde{V}_{0}, \tilde{V}_{0}^{2} \tilde{V}_{-1}^{3}\right) .
\end{aligned}
$$

At higher orders in $1 / \mathrm{m}$ the analysis has been carried out by Pineda (2002b). The same considerations as for the static limit apply here as far as the nontriangularity of the RG equations is concerned. In general, one has the structure

$$
\begin{aligned}
\nu_{u s} \frac{d}{d \nu_{u s}} \tilde{V}_{n}^{(\ell)} \sim & \sum_{\left.\left\{n_{i}\right\} \ell_{i}\right\}} \tilde{V}_{n_{1}}^{\left(\ell_{1}\right)} \tilde{V}_{n_{2}}^{\left(\ell_{2}\right)} \cdots \tilde{V}_{n_{j}}^{\left(\ell_{j}\right)}, \\
& \text { with } \sum_{i=1}^{j} \ell_{i}=\ell, \quad \sum_{i=1}^{j} n_{i}=n,
\end{aligned}
$$

and one has to pick up the leading contributions from all possible terms. Actually, as far as the NNLL heavyquarkonium mass is concerned, the relevant US running can be obtained by computing the diagram displayed in Eq. (133) (one also has to consider the running of $V_{A}$, which happens to be zero). Working in DR, one should note that the potentials have to be understood in $D$ dimensions [see, for instance, Eq. (3.1) of Schröder's thesis (1999a)]. Therefore powers of $g_{B}^{2}$ (the bare coupling) have dimensions and have to be compensated by powers

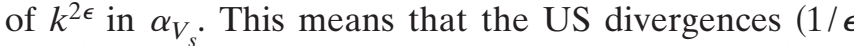
poles) generated by the right-hand side of Eq. (162) are absorbed by the terms in $\alpha_{V_{s}}$ proportional to $g_{B}^{8}$ or to a higher power. Finally, by solving Eq. (161) between $\nu_{s}$ and $\nu_{u s}$, we have $\tilde{V}\left(c\left(\nu_{s}\right), f\left(\nu_{p}, \nu_{s}\right), \nu_{s}, \nu_{u s}, r\right)$, where the running with respect to $\nu_{u s}$ is known. Note that the running with respect to $\nu_{s}$ is also known, since we demand that the potential be independent of it:

$$
\nu_{s} \frac{d}{d \nu_{s}} \tilde{V}=0,
$$

which can be solved by setting $\nu_{s}=1 / r$. Therefore one can also deduce the dependence of $\tilde{V}$ on $r$.

The final step is to obtain the RG equation for $\nu_{p}$. In pNRQCD, integrals over the relative three-momentum of the heavy quarks occur. When these integrals are finite no dependence on $\nu_{p}$ occurs and one has $|\mathbf{p}| \sim 1 / r$ $\sim m \alpha_{s}$ and $\mathbf{p}^{2} / m \sim m \alpha_{s}^{2}$. Therefore one can reduce $\nu_{u s}$ down to $\sim m \alpha_{s}^{2}$ reproducing the results obtained by Pineda (2002b). In general, the integrals over $\mathbf{p}$ are divergent, and the structure of the logarithms is dictated by the UV behavior of $\mathbf{p}$ and $1 / r$. This means that we cannot replace $1 / r$ and $\nu_{u s}$ by their physical expectation values but rather by their cutoffs within the integral over p, i.e., $\nu_{p}$. Therefore besides the potential's explicit de- pendence on $\nu_{p}$, which appears in $f$, it also implicitly depends on $\nu_{p}$ through the requirement $1 / r \sim|\mathbf{p}| \ll \nu_{p}$, and also through $\nu_{u s}$ since $\nu_{u s}$ has to fulfill $\mathbf{p}^{2} / m \ll \nu_{u s}$ $\ll|\mathbf{p}|$ in order to ensure that only soft degrees of freedom have been integrated out for a given $|\mathbf{p}|$. This latter requirement holds if we fix the final point of the evolution of the ultrasoft RG equation to be $\nu_{u s}=v_{p}^{2} / m$. At this stage, a single cutoff $\nu_{p}$ exists and the correlation of cutoffs becomes manifest. Therefore for the RG equation for $\nu_{p}$, the anomalous dimension of $\tilde{V}\left(c(1 / r), f\left(\nu_{p}, 1 / r\right), 1 / r, \nu_{p}^{2} / m, r\right)$ is at LO the same as the one of $\tilde{V}\left(c\left(\nu_{p}\right), f\left(\nu_{p}, \nu_{p}\right), \nu_{p}, \nu_{p}^{2} / m, \nu_{p}\right) .{ }^{15}$ It appears through the divergences induced by the iteration of the potentials in the way explained by Pineda (2002a) and Sec. IV.G. In particular, the computation of the anomalous dimension can be organized within an expansion in $\alpha_{s}$ and using the free propagators $G_{c}^{(0)}$. Finally, the running will go from $\nu_{p} \sim m$ down to $\nu_{p} \sim m \alpha_{s}$. A similar discussion applies to the running of the matching coefficients of the currents (or, in other words, of the imaginary terms of the potential). This completes the procedure to obtain the RG equations for the hard, soft, and US scales. An example is given below.

This line of investigation has led to several new results in heavy-quarkonium physics. They can be summarized as follows (we omit all numerical coefficients that can be found in the quoted literature):

- The NNLL correction to the heavy-quarkonium energy (Pineda, 2002b), i.e., corrections of order

$$
\delta E \sim m \alpha_{s}^{4}+m \alpha_{s}^{5} \ln \alpha_{s}+m \alpha_{s}^{6} \ln ^{2} \alpha_{s}+\cdots .
$$

- The LL (Pineda, 2002b) (first obtained by Hoang, Manohar, and Stewart, 2001) and NLL (Kniehl et al., 2004; Penin et al., 2004a) correction to the heavyquarkonium hyperfine splitting,

$$
\begin{aligned}
\delta E_{\mathrm{HF}} \sim & m \alpha_{s}^{4}+m \alpha_{s}^{5} \ln \alpha_{s}+m \alpha_{s}^{6} \ln ^{2} \alpha_{s}+\cdots+m \alpha_{s}^{5} \\
& +m \alpha_{s}^{6} \ln \alpha_{s}+m \alpha_{s}^{7} \ln ^{2} \alpha_{s}+m \alpha_{s}^{8} \ln ^{3} \alpha_{s}+\cdots
\end{aligned}
$$

$$
\begin{aligned}
& { }^{15} \text { Roughly speaking, this result can be thought of as expand- } \\
& \text { ing } \ln r \text { around } \ln \nu_{p} \text { in the potential, i.e., } \\
& \quad \tilde{V}\left(c(1 / r), f\left(\nu_{p}, 1 / r\right), 1 / r, \nu_{p}^{2} / m, r\right) \\
& \quad \simeq \tilde{V}\left(c\left(\nu_{p}\right), f\left(\nu_{p}, \nu_{p}\right), \nu_{p}, \nu_{p}^{2} / m, \nu_{p}\right)+\left.\ln \left(\nu_{p} r\right) r \frac{d}{d r} \tilde{V}\right|_{1 / r=\nu_{p}}+\cdots
\end{aligned}
$$

The $\ln \left(\nu_{p} r\right)$ terms give subleading contributions to the anomalous dimension when introduced in divergent integrals over $\mathbf{p}$. An explicit example of this type of correction appears in the computation of the hyperfine splitting of heavy quarkonium at NLL (Kniehl et al., 2004; Penin et al., 2004a). 
- The NLL (Pineda, 2002a) correction to the inclusive electromagnetic decays (this result can be applied to $\bar{t}-t$ production at threshold or NR sum rules since the running of the electromagnetic current matching coefficient is the only nontrivial object that appears in the NLL running),

$$
\begin{aligned}
\Gamma\left(V_{Q}(n S) \rightarrow e^{+} e^{-}\right) \sim & m \alpha_{s}^{3}\left(1+\alpha_{s}^{2} \ln \alpha_{s}+\alpha_{s}^{3} \ln ^{2} \alpha_{s}\right. \\
+\cdots), & \\
\Gamma\left(P_{Q}(n S) \rightarrow \gamma \gamma\right) \sim & m \alpha_{s}^{3}\left(1+\alpha_{s}^{2} \ln \alpha_{s}+\alpha_{s}^{3} \ln ^{2} \alpha_{s}+\cdots\right),
\end{aligned}
$$

and for the ratio the NNLL correction (Penin et al., 2004b)

$$
\begin{array}{r}
\frac{\Gamma\left(V_{Q}(n S) \rightarrow e^{+} e^{-}\right)}{\Gamma\left(P_{Q}(n S) \rightarrow \gamma \gamma\right)} \sim 1+\alpha_{s}^{2} \ln \alpha_{s}+\alpha_{s}^{3} \ln ^{2} \alpha_{s}+\cdots \\
+\alpha_{s}^{3} \ln \alpha_{s}+\alpha_{s}^{4} \ln ^{2} \alpha_{s}+\cdots
\end{array}
$$

The resummation of logarithms using EFTs was first addressed within the velocity NRQCD framework (Luke et al., 2000; see also Manohar and Stewart, 2000c, 2001; Hoang, Manohar, and Stewart, 2001; Hoang and Stewart, 2003; Hoang, 2004), where the relevance of the cutoff correlation for the RG was first realized. Nevertheless, the early formulations of this theory had some problems (in particular, concerning the treatment of US modes), which led to incorrect results for the heavyquarkonium mass at NNLL (Hoang, Manohar, and Stewart, 2001) and the electromagnetic-current matching coefficient at NLL (Manohar and Stewart, 2001). They have been resolved by Hoang and Stewart (2003) and their results now agree with those obtained in pNRQCD (Pineda, 2002a, 2002b). The application of the RG to QED bound states has also been considered in both formalisms; see Manohar and Stewart (2000a); Pineda (2002a, 2002c); Penin et al. (2004b).

Finally, we illustrate the method in the simplest possible situation where all the scales appear. We consider the corrections to the heavy-quarkonium spectrum for the non-equal-mass case in the limit where one of the masses $\left(m_{2}\right)$ goes to infinity, and in the Abelian limit with zero light flavors $\left(C_{F} \rightarrow 1, C_{A} \rightarrow 0, T_{F} \rightarrow 1, n_{f} \rightarrow 0\right)$. This is nothing but the hydrogen-atom case. We compute some NNNLL corrections to the Lamb shift of $\mathcal{O}\left(m \alpha_{s}^{8} \ln ^{3} \alpha_{s}\right)$, which were first computed using the RG by Manohar and Stewart (2000a). Here we follow the discussion in the articles of Pineda (2002a, 2002c). In this limit, $\alpha_{s}$ does not run and we can neglect the fourfermion matching coefficients since they are suppressed by powers of $1 / m_{2}$. Therefore we only have to consider the running of the matching coefficients of the heavyquark bilinear terms. At $\mathcal{O}\left(1 / \mathrm{m}^{2}\right), c_{D}$ is the only matching coefficient with nontrivial running. By solving Eq. (35) in this limit, one obtains

$$
c_{D}\left(\nu_{s}\right)=1-\frac{8}{3} \frac{\alpha_{s}}{\pi} \ln \frac{\nu_{s}}{m} .
$$

At the pNRQED level, we have to consider first the US RG running of $D_{d, s}^{(2)}$, which follows from Eq. (133). It reads (we use $V_{A}=1$ and $c_{S}^{(1,-2)}=1$ )

$$
\nu_{u s} \frac{d}{d \nu_{u s}} D_{d, s}^{(2)}=-\frac{4}{3} \frac{\alpha_{s}^{2}}{\pi} \text {. }
$$

By using the initial matching condition

$$
D_{d, s}^{(2)}\left(\nu_{s}\right)=\alpha_{s} \frac{c_{D}\left(\nu_{s}\right)}{2},
$$

we can solve Eq. (171). The solution is

$$
D_{d}^{(2)}\left(\nu_{u s}\right)=\frac{\alpha_{s}}{2}\left(1-\frac{8}{3} \frac{\alpha_{s}}{\pi} \ln \frac{\nu_{u s}}{m}\right),
$$

which gives the full NNLL contribution to the spectrum of $\mathcal{O}\left(m \alpha_{s}^{5} \ln \alpha_{s}\right)$ and nothing else. At NNNLL, we can obtain the $\mathcal{O}\left(m \alpha_{s}^{8} \ln ^{3} \alpha_{s}\right)$ contribution from Eq. (137), which is due to the diagram in Fig. 12. This is because the $\mathcal{O}\left(m \alpha_{s}^{8} \ln ^{3} \alpha_{s}\right)$ term has the highest power of logarithm that could appear from this evaluation of the energy and that in order to achieve such power it is necessary to mix with its NNLL-terms. As we have seen, the latter only appear in the LL evaluation of $D_{d}^{(2)}(173)$, which, indeed, only produces a single logarithm. The other point is that the NLL evaluation of the potentials only produces single logarithms unless mixed with LL running. Therefore the diagrams with the highest power of $D_{d}^{(2)}$ will give the highest logarithmic power in the spectrum of the NNNLL. Thus we only have to solve Eq. (137) (note the replacement $\nu_{u s}=\nu_{p}^{2} / m$ ), which in the limit considered here reads

$$
\nu_{p} \frac{d}{d \nu_{p}} D_{d, s}^{(2)}\left(\nu_{p}\right)=\alpha_{s} D_{d, s}^{(2) 2}\left(\nu_{p}\right)+\cdots .
$$

The solution is

$$
\delta D_{d}^{(2)}=\frac{64}{27} \alpha_{s}^{3}\left(\frac{\alpha_{s}}{\pi}\right)^{2} \ln ^{3} \frac{\nu_{p}}{m} .
$$

\section{RENORMALONS AND THE DEFINITION OF THE HEAVY-QUARK MASS}

\section{A. The pole mass and static singlet potential renormalon}

The pole mass of a heavy quark can be related to the MS mass by the series

$$
m=m_{\overline{\mathrm{MS}}}+\sum_{n=0}^{\infty} r_{n} \alpha_{s}^{n+1},
$$

where $\alpha_{s} \equiv \alpha_{s}(\nu), m_{\overline{\mathrm{MS}}}$ is calculated at the normalization point $\nu=m_{\overline{\mathrm{MS}}}$ (in this way logarithms that are not associated with the renormalon are resummed), and the first three coefficients $r_{0}, r_{1}$, and $r_{2}$ are known (Gray et al., 1990; Chetyrkin and Steinhauser, 2000; Melnikov and 
Ritbergen, 2000). The pole mass is also known to be IR finite and scheme independent at any finite order in $\alpha_{s}$ (Kronfeld, 1998). We then define the Borel transform

$$
m=m_{\overline{\mathrm{MS}}}+\int_{0}^{\infty} d t e^{-t / \alpha_{s}} B[m](t), \quad B[m](t) \equiv \sum_{n=0}^{\infty} r_{n} \frac{t^{n}}{n !} .
$$

We denote by renormalons the singularities on the real axis of the Borel plane. ${ }^{16}$ The perturbative expansion behavior of Eq. (176) at large orders is dictated by the closest renormalon to the origin of its Borel transform, which happens to be located at $t=2 \pi / \beta_{0}$ (Beneke and Braun, 1994; Bigi et al., 1994; Neubert and Sachrajda, 1995). More precisely, the behavior of the Borel transform near the closest renormalon at the origin is (we define $\left.u=\beta_{0} t / 4 \pi\right)$

$$
\begin{aligned}
B[m](t(u))= & B\left[\delta m_{\mathrm{RS}}\right](t(u)) \\
& +(\text { term analytic at } u=1 / 2),
\end{aligned}
$$

where

$$
\begin{aligned}
B\left[\delta m_{\mathrm{RS}}\right](t(u)) \equiv & N_{m} \nu \frac{1}{(1-2 u)^{1+b}}\left[1+c_{1}(1-2 u)\right. \\
& \left.+c_{2}(1-2 u)^{2}+\cdots\right] .
\end{aligned}
$$

This dictates that the behavior of the perturbative expansion at large orders be

$$
\begin{aligned}
r_{n}= & N_{m} \nu\left(\frac{\beta_{0}}{2 \pi}\right)^{n} \frac{\Gamma(n+1+b)}{\Gamma(1+b)}\left(1+\frac{b}{(n+b)} c_{1}\right. \\
& \left.+\frac{b(b-1)}{(n+b)(n+b-1)} c_{2}+\cdots\right) .
\end{aligned}
$$

The different $b, c_{1}, c_{2}$, etc. can be obtained from the procedure used by Beneke (1995). The coefficients $b$ and $c_{1}$ were computed by Beneke (1995), and $c_{2}$ by Beneke (1999) and Pineda (2001). They are

$$
b=\frac{\beta_{1}}{2 \beta_{0}^{2}}, \quad c_{1}=\frac{1}{4 b \beta_{0}^{3}}\left(\frac{\beta_{1}^{2}}{\beta_{0}}-\beta_{2}\right),
$$

and

$$
\begin{aligned}
c_{2}= & \frac{1}{b(b-1)} \\
& \times \frac{\beta_{1}^{4}+4 \beta_{0}^{3} \beta_{1} \beta_{2}-2 \beta_{0} \beta_{1}^{2} \beta_{2}+\beta_{0}^{2}\left(-2 \beta_{1}^{3}+\beta_{2}^{2}\right)-2 \beta_{0}^{4} \beta_{3}}{32 \beta_{0}^{8}} .
\end{aligned}
$$

Approximate determinations for $N_{m}$ have been obtained by Pineda (2001); Lee (2003b); Cvetic (2004); see also Pineda (2003b).

One can perform the same analysis with the singlet static potential when $\Lambda_{\mathrm{QCD}} \ll 1 / r$. Its perturbative expansion reads

\footnotetext{
${ }^{16}$ We shall not consider singularities due to instantons (Le Guillou and Zinn-Justin 1990).
}

$$
V_{s}^{(0)}\left(r ; \nu_{u s}\right)=\sum_{n=0}^{\infty} V_{s, n}^{(0)} \alpha_{s}^{n+1}
$$

The potential, however, is not an IR safe object since it depends on the IR cutoff $\nu_{u s}$, which first appears at $\mathcal{O}\left(\alpha_{s}^{4}\right)$ (for more details see Sec. IV.F). Nevertheless, these US logarithms are not associated with the first IR renormalon since they also appear in momentum space (see also the discussion below). They will not be considered further in this section.

We now use the observation that the first IR renormalon of the singlet static potential cancels with (twice) the renormalon of the pole mass. This has been proven in the (one-chain) large- $\beta_{0}$ approximation by Pineda (1998) and Hoang, Smith, et al. (1999b) and at any loop (disregarding possible effects due to $\nu_{u s}$ ) by Beneke (1998). It can also be argued to hold from an EFT approach where any renormalon ambiguity should cancel between operators and matching coefficients. Let us consider, for instance, $1 / r \gg \Lambda_{\mathrm{QCD}}$. If we understand the quantity $2 m$ $+V_{s}^{(0)}$ as an observable up to $\mathcal{O}\left(r^{2} \Lambda_{\mathrm{QCD}}^{3}, \Lambda_{\mathrm{QCD}}^{2} / m\right)$ renormalon (and/or nonperturbative) contributions, then this proves the (first IR) renormalon cancellation at any loop (as well as the IR-renormalon independence of $\nu_{u s}$ ).

One can now read off the asymptotic behavior of the static potential from the one of the pole mass and work analogously. We define the Borel transform

$$
V_{s}^{(0)}=\int_{0}^{\infty} d t e^{-t / \alpha_{s}} B\left[V_{s}^{(0)}\right](t), \quad B\left[V_{s}^{(0)}\right](t) \equiv \sum_{n=0}^{\infty} V_{s, n}^{(0)} \frac{t^{n}}{n !} .
$$

The closest renormalon to the origin is located at $t$ $=2 \pi / \beta_{0}$. This dictates that the behavior of the perturbative expansion at large orders be

$$
\begin{aligned}
V_{s, n}^{(0)}= & N_{V_{s}} \nu\left(\frac{\beta_{0}}{2 \pi}\right)^{n} \frac{\Gamma(n+1+b)}{\Gamma(1+b)}\left(1+\frac{b}{(n+b)} c_{1}\right. \\
& \left.+\frac{b(b-1)}{(n+b)(n+b-1)} c_{2}+\cdots\right),
\end{aligned}
$$

and the Borel transform near the singularity reads

$$
\begin{aligned}
B\left[V_{s}^{(0)}\right](t(u))= & N_{V_{s}} \nu \frac{1}{(1-2 u)^{1+b}}\left[1+c_{1}(1-2 u)+c_{2}(1\right. \\
& \left.-2 u)^{2}+\cdots\right]+(\text { analytic term }) .
\end{aligned}
$$

In this case, by analytic term we mean an analytic function up to the next IR renormalon at $u=3 / 2$ (Aglietti and Ligeti, 1995).

For $N_{V_{s}}$ some approximate determinations exist (Pineda, 2001; Lee, 2003b; see also Pineda, 2003b). Actually, the best determinations come from $N_{m}$ using the cancellation of the pole mass and static singlet potential renormalon, i.e.,

$$
2 N_{m}+N_{V_{s}}=0 .
$$




\section{B. Renormalon-subtracted scheme and power counting}

In EFTs with heavy quarks, the inverse of the heavyquark mass becomes one of the expansion parameters (and of the matching coefficients). A natural choice in the past has been the pole mass because it is the natural definition in processes where the particles eventually measured in the detectors correspond to the fields in the Lagrangian (as in QED). This is not the case in QCD. One consequence of this is that the pole mass suffers from renormalon singularities. Moreover, since these renormalon singularities lie close to the origin of the Borel plane and perturbative calculations have gone very far for systems with heavy quarks, they manifest themselves as a poor convergence of the perturbative series. It is then natural to try to define a new mass parameter, which replaces the pole mass, but is still adequate for threshold problems. Several choices have been proposed in the literature: the kinetic mass (Bigi et al., 1994), the potential-subtracted (PS) mass (Beneke, 1998), the $1 S$ mass (Hoang, Ligeti, and Manohar, 1999), the $\overline{\text { PS}}$-mass (Yakovlev and Groote, 2001), and the RS (RS) mass (Pineda, 2001). All of them achieve the renormalon cancellation and share the following structure:

$$
m_{X}=m-\delta m_{X}
$$

where $X=\{\mathrm{PS}, 1 S, \ldots\}$ and $\delta m_{X}$ is an object such that

$B\left[\delta m_{X}\right]=B\left[\delta m_{\mathrm{RS}}\right]+($ analytic term at $u=1 / 2)$.

The different definitions have different analytic terms. $\delta m_{\text {kin }}$ is defined as the self-energy of a static quark computed with a hard cutoff, $\delta m_{\mathrm{PS}}$ is defined as $1 / 2$ the selfenergy of the Coulomb potential computed with a hard cutoff much smaller than $1 / r, \delta m_{\mathrm{PS}}$ is defined as the soft part of the heavy-quark self-energy computed with a hard cutoff, and $\delta m_{1 S}$ is $1 / 2$ the perturbative binding energy of the ground state of heavy quarkonium (note that in this case the renormalon cancellation is achieved between different powers of $\alpha_{s}$ ). We shall not discuss further all these threshold masses. Instead, we focus on one, the RS mass, which better matches with the analyses of the previous section. In any case, a large part of the discussion also holds when replacing RS by $X$. It should be noted that since different masses implement the renormalon cancellation in different ways, different systematic errors appear. For instance, the major error in the RS mass comes from $N_{m}$ [see Eq. (180)]. For the kinetic and PS masses, it seems difficult to compute higher-order terms. The PS and $1 S$ masses depend on the US scale at NNNLO, which may be problematic once this precision is needed (for instance, in $B$ physics). Finally, the $1 S$ mass assumes that the ground state of heavy quarkonium is mainly a perturbative system. Therefore having at one's disposal several masses may help to better handle the errors, e.g., in the extraction of the MS quark masses.

The RS definition tries to cancel the poor perturbative behavior associated with the renormalon, which is due to the nonanalytic terms in $1-2 u$ in the Borel transform of the pole mass. These terms also exist in the effective theory. Therefore the procedure followed by Pineda (2001) was to subtract the pure renormalon contribution in the new mass definition, ${ }^{17}$ the RS mass $m_{\mathrm{RS}}$,

$$
\begin{aligned}
m_{\mathrm{RS}}\left(\nu_{f}\right)= & m-\sum_{n=1}^{\infty} N_{m} \nu_{f}\left(\frac{\beta_{0}}{2 \pi}\right)^{n} \alpha_{s}^{n+1}\left(\nu_{f}\right) \\
& \times \sum_{k=0}^{\infty} c_{k} \frac{\Gamma(n+1+b-k)}{\Gamma(1+b-k)},
\end{aligned}
$$

where $c_{0}=1$. We expect that with this renormalon-free definition, the coefficients multiplying the expansion parameters in the effective-theory calculation will have a natural size and that the same holds for the coefficients multiplying the powers of $\alpha_{s}$ in the perturbative expansion relating $m_{\mathrm{RS}}$ to $m_{\overline{\mathrm{MS}}}$. Therefore we do not lose accuracy if we first obtain $m_{\mathrm{RS}}$ and later on use the perturbative relation between $m_{\mathrm{RS}}$ and $m_{\mathrm{MS}}$ in order to obtain the latter. Nevertheless, since we work order by order in $\alpha_{s}$, in the relation between $m_{\mathrm{RS}}$ and $m_{\mathrm{MS}}$ it is important to expand everything in terms of $\alpha_{s}$, specifically $\alpha_{s}\left(\nu_{f}\right)$, in order to achieve the renormalon cancellation order by order in $\alpha_{s}$. Then, the perturbative expansion in terms of the $\overline{\mathrm{MS}}$ mass reads

$$
m_{\mathrm{RS}}\left(\nu_{f}\right)=m_{\mathrm{MS}}+\sum_{n=0}^{\infty} r_{n}^{\mathrm{RS}} \alpha_{s}^{n+1}
$$

where $r_{n}^{\mathrm{RS}}=r_{n}^{\mathrm{RS}}\left(m_{\mathrm{MS}}, \nu, \nu_{f}\right)$. These $r_{n}^{\mathrm{RS}}$ are the ones expected to be of natural size (or at least not to be artificially enlarged by the first IR renormalon).

These definitions significantly improve the convergence of the perturbative series in comparison with the pole mass. We refer the reader to the work of Pineda (2001) for numerical details.

The shift from the pole mass to the RS mass affects the explicit expression of the effective Lagrangians. In particular, in HQET at LO, a residual mass term appears in the Lagrangian

$$
\mathcal{L}=\bar{h}\left(i D_{0}-\delta m_{\mathrm{RS}}\right) h+\mathcal{O}\left(\frac{1}{m_{\mathrm{RS}}}\right),
$$

where $\delta m_{\mathrm{RS}}=m-m_{\mathrm{RS}}$ and similarly for the NRQCD Lagrangian.

If we consider the LO in $1 / m$ for pNRQCD when $\Lambda_{\mathrm{QCD}} \ll m \alpha_{s}$, the residual mass term is absorbed into the static potential (in going from NRQCD to pNRQCD, one runs down the scale $\nu_{f}$ to $\nu_{f} \lessgtr m \alpha_{s}$ ). We can then, analogous to the RS mass, define a singlet static RS potential

$$
V_{s, \mathrm{RS}}^{(0)}\left(\nu_{f}\right)=V_{s}^{(0)}+2 \delta m_{\mathrm{RS}},
$$

where the coefficients multiplying the perturbative series should be of $\mathcal{O}(1)$ (provided that we expand $V_{s}^{(0)}$ and

\footnotetext{
${ }^{17}$ One could also choose not to include terms proportional to $c_{n}$ for $n \geqslant 2$ since these terms actually go to zero for $u \rightarrow 1 / 2$ for the physical value of $b \sim 0.4$.
} 
$\delta m_{\mathrm{RS}}$ in the same parameter, namely, $\left.\alpha_{s}\right)$. Notice also the trivial fact that the scheme dependence of $m_{\mathrm{RS}}$ cancels with the scheme dependence of $V_{\mathrm{RS}}$. This definition significantly improves the perturbative expansion in the potential. For a numerical analysis we refer the reader to the work of Pineda (2001, 2003b).

The pNRQCD Lagrangian for weak coupling in the RS scheme is formally equal to the one in the on-shell scheme [see Eq. (60)] with the modifications $m_{1(2)}$ $\rightarrow m_{1, \mathrm{RS}(2, \mathrm{RS})}, V \rightarrow V_{\mathrm{RS}}$, and so on. Note, in particular, that now the expansion is in terms of $1 / m_{\mathrm{Rs}}{ }^{18}$ One can then compute observables along the lines of Sec. IV.G (at the practical level one can work with the pole mass and change to the RS mass at the end). For instance, one would obtain the following expression for the heavyquarkonium spectrum [see Eq. (138)]:

$$
M_{n l j}=2 m_{\mathrm{RS}}+\sum_{m=2}^{\infty} A_{n l j}^{m, \mathrm{RS}}\left(\nu_{u s}\right) \alpha_{s}^{m}+\delta M_{n l j}^{\mathrm{US}}\left(\nu_{u s}\right),
$$

where the $\nu_{u s}$ scale dependence of the different pieces cancels in the overall sum (for the perturbative sum, this dependence first appears in $A_{n l j}^{5, \mathrm{RS}}$ ).

We expect that by working with the RS scheme the coefficients multiplying the powers of $\alpha_{s}$ will now be of natural size and therefore the convergence is improved compared with the on-shell scheme. Actually, this appears to be the case. See Sec. VIII for details and a phenomenological discussion.

Finally, we discuss some theoretical issues (see also Beneke, 1999). First, once one agrees to give up using the pole mass as an expansion parameter, one may still wonder why not use the $\overline{\mathrm{MS}}$ mass instead. There are several answers to this question. One is that due to the fact that there is another scale, $m \alpha_{s}$, besides $m$, one would not achieve the renormalon cancellation order by order in $\alpha_{s}$ but rather between different orders in $\alpha_{s}$, jeopardizing in this way the convergence of the perturbative expansion. This can be resolved by using the upsilon expansion (Hoang, Ligeti, and Manohar, 1999). Nevertheless, some other problems may remain. Working with $m_{\overline{\mathrm{MS}}}$ would mean introducing a large shift in the pNRQCD Lagrangian of $\mathcal{O}\left(m \alpha_{s}\right)$ thereby compromising the power-counting rules. ${ }^{19}$ Furthermore, by expanding everything in terms of $\alpha_{s}$, we may introduce a potentially

\footnotetext{
${ }^{18}$ Note that the definition of the RS scheme in the octet sector is more involved since there are some renormalons left at $u$ $=1 / 2$ in $V_{o}^{(0)}+2 \delta m_{\mathrm{RS}}$. The reason is that even at LO in $1 / m$, $2 m+V_{o}^{(0)}$ is not an observable due to the fact that there is still interaction with low-energy gluons. Therefore one expects $2 m+V_{o}^{(0)}$ to be ambiguous by an amount of $\mathcal{O}\left(\Lambda_{\mathrm{QCD}}\right)$. We elaborate on this in section VI.

${ }^{19}$ This is certainly so for $t-\bar{t}$ physics. Nevertheless, for the bottom quark, the $\mathcal{O}\left(m \alpha_{s}\right)$ term does not seem to be that large numerically, being much smaller than the typical values of the soft scale in the $Y(1 S)$. Therefore it may be that working with the MS mass does not destroy the power-counting rules or pNRQCD (or HQET) at the practical level.
}

large logarithm, $\ln m / \nu$ [note that we cannot minimize this logarithm except at the price of introducing another large logarithm, $\left.\ln \left(m \alpha_{s} / \nu\right)\right]$.

\section{VI. (P)NRQCD: THE STATIC LIMIT}

Although NRQCD and PNRQCD were originally designed to study $Q-\bar{Q}$ systems of large but finite mass, it is very interesting to consider their static limit (where $m$ $\rightarrow \infty$ while keeping all the other scales finite). On the one hand, the static energy spectra are the main ingredients for the potentials both in the strong- and in the weakcoupling regimes. On the other hand, the study of the energy spectrum is interesting in itself. For instance, a linear dependence on $r$ for the ground-state energy at long distances is usually considered a proof of confinement. The abundant lattice data (at least of quenched simulations) make it possible to study quantitatively for which distances the potentials are in the perturbative or nonperturbative regime, providing a controlled framework for discerning when to use the weak- or the strongcoupling version, of pNRQCD. To answer this question, the proper handling of the renormalon singularities will be crucial.

\section{A. NRQCD in the static limit}

The Hamiltonian associated with the Lagrangian (4) is

$$
\begin{aligned}
& H=H^{(0)}+\mathcal{O}(1 / m), \\
& H^{(0)}=\int d^{3} \mathbf{x} \frac{1}{2}\left(\boldsymbol{\Pi}^{a} \boldsymbol{\Pi}^{a}+\mathbf{B}^{a} \mathbf{B}^{a}\right)-\sum_{j=1}^{n_{f}} \int d^{3} \mathbf{x} \bar{q}_{j} i \mathbf{D} \cdot \gamma q_{j},
\end{aligned}
$$

and the physical states are constrained to satisfy the Gauss law:

$\mathbf{D} \cdot \boldsymbol{\Pi}^{a} \mid$ phys $\rangle=g\left(\psi^{\dagger} T^{a} \psi+\chi^{\dagger} T^{a} \chi+\sum_{j=1}^{n_{f}} \bar{q}_{j} \gamma^{0} T^{a} q_{j}\right) \mid$ phys $\rangle$.

We are interested in the one-quark-one-antiquark sector of the Fock space. In the static limit it is spanned by

$$
\left|\underline{\mathrm{n}} ; \mathbf{x}_{1}, \mathbf{x}_{2}\right\rangle^{(0)} \equiv \psi^{\dagger}\left(\mathbf{x}_{1}\right) \chi_{c}^{\dagger}\left(\mathbf{x}_{2}\right)\left|n ; \mathbf{x}_{1}, \mathbf{x}_{2}\right\rangle^{(0)}, \quad \forall \mathbf{x}_{1}, \mathbf{x}_{2},
$$

where $\left|\underline{\mathrm{n}} ; \mathbf{x}_{1}, \mathbf{x}_{2}\right\rangle^{(0)}$ is a gauge-invariant (since it satisfies the Gauss law) eigenstate (up to a phase) of $H^{(0)}$ with energy $E_{n}^{(0)}\left(\mathbf{x}_{1}, \mathbf{x}_{2}\right)$. For convenience, we use here the field $\chi_{c}(\mathbf{x})=i \sigma^{2} \chi^{*}(\mathbf{x})$, instead of $\chi(\mathbf{x})$, because it is the one to which a particle interpretation can easily be given: it corresponds to a Pauli spinor that annihilates a fermion in the $3^{*}$ representation of color $\mathrm{SU}(3)$ with the standard, particlelike, spin structure. $\left|n ; \mathbf{x}_{1}, \mathbf{x}_{2}\right\rangle^{(0)}$ encodes the gluonic content of the state, namely, it is annihilated by $\chi_{c}(\mathbf{x})$ and $\psi(\mathbf{x})$ for all $\mathbf{x}$. It transforms as a $3_{\mathbf{x}_{1}} \otimes 3_{\mathbf{x}_{2}}^{*}$ under 


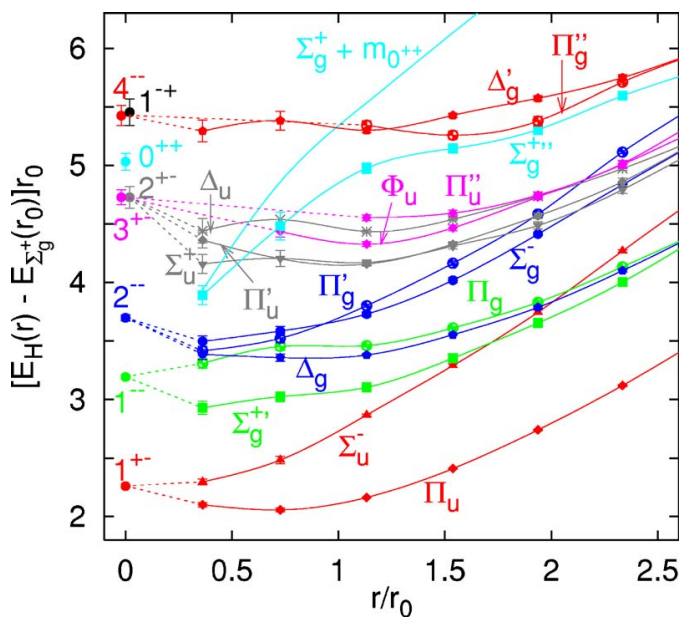

FIG. 13. (Color online) Different hybrid potentials (Juge et al., 2003) at a lattice spacing $a_{\sigma} \approx 0.2 \mathrm{fm} \approx 0.4 r_{0}$, where $r_{0} \approx 0.5 \mathrm{fm}$ is the scale for which $-r_{0}^{2} d V /\left.d r\right|_{r=r_{0}}=1.65$ (Sommer, 1994), in comparison with the gluelump spectrum (Foster and Michael, 1999) (circles, left-most data points). The gluelump spectrum has been shifted by a constant to adjust the $1^{+-}$state with the $\Pi_{u}$ and $\Sigma_{u}^{-}$potentials at short distance. In addition, we include the sum of the ground-state $\left(\Sigma_{g}^{+}\right)$potential and the scalar glueball mass $m_{0^{++}}$(Bali et al., 1993; Morningstar and Peardon, 1999; Lucini and Teper, 2001). The lines are drawn to guide the eye. From Bali and Pineda, 2004.

color SU(3). The normalizations are taken as follows:

$$
\begin{aligned}
& { }^{(0)}\left\langle m ; \mathbf{x}_{1}, \mathbf{x}_{2} \mid n ; \mathbf{x}_{1}, \mathbf{x}_{2}\right\rangle^{(0)}=\delta_{n m}, \\
& { }^{(0)}\left\langle\underline{\mathrm{m}} ; \mathbf{x}_{1}, \mathbf{x}_{2} \mid \underline{\mathbf{n}} ; \mathbf{y}_{1}, \mathbf{y}_{2}\right\rangle^{(0)}=\delta_{n m} \delta^{(3)}\left(\mathbf{x}_{1}-\mathbf{y}_{1}\right) \delta^{(3)}\left(\mathbf{x}_{2}-\mathbf{y}_{2}\right) .
\end{aligned}
$$

We have made explicit that the positions $\mathbf{x}_{1}$ and $\mathbf{x}_{2}$ of the quark and antiquark, respectively, are good quantum numbers for the static solution $\left|\underline{n} ; \mathbf{x}_{1}, \mathbf{x}_{2}\right\rangle^{(0)}$ (since there are no spatial derivatives in the Lagrangian), whereas $n$ generically denotes the remaining quantum numbers. We also choose the basis such that $T\left|\underline{n} ; \mathbf{x}_{1}, \mathbf{x}_{2}\right\rangle^{(0)}$ $=\left|\underline{\underline{n}} ; \mathbf{x}_{1}, \mathbf{x}_{2}\right\rangle^{(0)}$, where $T$ is the time-reversal operator. The ground-state energy $E_{0}^{(0)}\left(\mathbf{x}_{1}, \mathbf{x}_{2}\right)$ can be associated with the static potential of the heavy quarkonium in some circumstances (see Sec. VII.E). The remaining energies $E_{n}^{(0)}\left(\mathbf{x}_{1}, \mathbf{x}_{2}\right), n \neq 0$, are usually associated with the potential used in order to describe hybrids (they may also correspond to heavy quarkonium or heavy hybrids plus glueballs). They can be computed on the lattice (see Juge et al., 2003, and also Fig. 13). Translational invariance implies that $E_{n}^{(0)}\left(\mathbf{x}_{1}, \mathbf{x}_{2}\right)=E_{n}^{(0)}(r)$. This means that they are functions of $r$ and the only other scale in the system, $\Lambda_{\mathrm{QCD}}$.

In static NRQCD, the gluonic excitations between static quarks have the same symmetries as in a diatomic molecule (see Messiah, 1979). In the center-of-mass system, these correspond to the symmetry group $D_{\infty h}$ (replacing the parity generator by $C P$ ). According to that symmetry, the mass eigenstates are classified in terms of the angular momentum along the quark-antiquark axis
$\left(\left|L_{z}\right|=0,1,2, \ldots\right.$ to which one gives the traditional names $\Sigma, \Pi, \Delta, \ldots), C P$ (even, $g$, or odd, $u$ ), and the reflection properties with respect to a plane that passes through the quark-antiquark axis (even, + , or odd, - ). Only the $\Sigma$ states are not degenerate with respect to the reflection symmetry.

\section{B. Static pNRQCD in the weak-coupling regime}

In the static limit pNRQCD has the same symmetries as NRQCD. In this section we discuss some general properties of the short-distance behavior of the static energies that can be straightforwardly derived within this EFT. We shall follow the work of Brambilla et al. (2000).

In the limit $\Lambda_{\mathrm{QCD}} \ll 1 / r$ and at LO in $1 / m$, the spectrum of the theory can be read off from the Lagrangian (60). In particular, the LO solution corresponds to the zeroth order of the multipole and $1 / \mathrm{m}$ expansions. At this order, while the singlet decouples from the octet and the gluons, the octet is still coupled to gluons. We call the states made of an adjoint source in the presence of a gluonic field gluelumps,

$$
H(\mathbf{R}, \mathbf{r}, t) \equiv H^{a}(\mathbf{R}, t) O^{a}(\mathbf{R}, \mathbf{r}, t) .
$$

These, in turn, correspond to the gluonic excitations between static quarks in the short-distance limit, for which there is abundant nonperturbative data available from lattice simulations (see Fig. 13). Depending on the glue operator $H^{a}$ and its symmetries, the gluelump operator $O^{a} H^{a}$ describes a specific gluonic excitation between static quarks and its static energy $V_{H}$.

In static pNRQCD at lowest order in the multipole expansion, besides the symmetries of static NRQCD, extra symmetries for the gluonic excitations between static quarks appear. The glue dynamics no longer involves the relative coordinate $\mathbf{r}$. Therefore the glue associated with a gluonic excitation between static quarks acquires a spherical symmetry. In the center-of-mass system, gluonic excitations between static quarks are therefore classified according to representations of $O(3) \otimes \mathrm{C}$, which we summarize by $L$, the angular momentum, $C P$, and reflection with respect to a plane passing through the quark-antiquark axis. Since this symmetry group is larger than that of NRQCD, several gluonic excitations between static quarks are expected to be approximately degenerate in pNRQCD, i.e., in the short-distance limit $r \ll 1 / \Lambda_{\mathrm{QCD}}$. We illustrate this point in Table I where all operators $H$ up to dimension 3 are built and classified according to their quantum numbers in NRQCD and pNRQCD. In Table I all the operators are evaluated at the center-of-mass coordinates. $\Sigma_{g}^{+}$is not displayed since it corresponds to the singlet state. The prime indicates excited states of the same quantum numbers. The operators chosen for the $\Pi$ and $\Delta$ states are not eigenstates of the reflection operator. This is not important since these states are degenerate with respect to this symmetry. From the results of Table I the following degeneracies are expected in the short-distance limit: 
TABLE I. Operators $H$ for the $\Sigma, \Pi$, and $\Delta$ gluonic excitations between static quarks in pNRQCD up to dimension 3. The covariant derivative is understood in the adjoint representation. $\mathbf{D} \cdot \mathbf{B}$ and $\mathbf{D} \cdot \mathbf{E}$ do not appear, the first because it is identically zero after using the Jacobi identity, while the second gives vanishing contributions after using the equations of motion. From Brambilla et al., 2000.

\begin{tabular}{ccc}
\hline \hline $\begin{array}{c}\text { Gluelumps } \\
O^{a} H^{a}\end{array}$ & $L=1$ & $L=2$ \\
\hline$\Sigma_{g}^{+\prime}$ & $\mathbf{r} \cdot \mathbf{E}, \mathbf{r} \cdot(\mathbf{D} \times \mathbf{B})$ & \\
$\Sigma_{g}^{-}$ & & $(\mathbf{r} \cdot \mathbf{D})(\mathbf{r} \cdot \mathbf{B})$ \\
$\Pi_{g}$ & $\mathbf{r} \times \mathbf{E}, \mathbf{r} \times(\mathbf{D} \times \mathbf{B})$ & \\
$\Pi_{g}^{\prime}$ & & $(\mathbf{r} \times \mathbf{D})^{i}(\mathbf{r} \times \mathbf{B})^{j}+(\mathbf{r} \times \mathbf{D})^{j}(\mathbf{r} \times \mathbf{B})^{i}$ \\
$\Delta_{g}$ & & $(\mathbf{r} \cdot \mathbf{D})(\mathbf{r} \cdot \mathbf{E})$ \\
$\Sigma_{u}^{+}$ & & \\
$\Sigma_{u}^{-}$ & $\mathbf{r} \cdot \mathbf{B}, \mathbf{r} \cdot(\mathbf{D} \times \mathbf{E})$ & $\mathbf{r} \cdot \mathbf{B})]$ \\
$\Pi_{u}$ & $\mathbf{r} \times \mathbf{B}, \mathbf{r} \times(\mathbf{D} \times \mathbf{E})$ & $(\mathbf{r} \times \mathbf{D})^{i}(\mathbf{r} \times \mathbf{E})^{j}+(\mathbf{r} \times \mathbf{D})^{j}(\mathbf{r} \times \mathbf{E})^{i}$ \\
$\Pi_{u}^{\prime}$ & & \\
$\Delta_{u}$ & & \\
\hline \hline
\end{tabular}

$$
\begin{aligned}
& \Sigma_{g}^{+\prime} \sim \Pi_{g}, \quad \Sigma_{g}^{-} \sim \Pi_{g}^{\prime} \sim \Delta_{g}, \\
& \Sigma_{u}^{-} \sim \Pi_{u}, \quad \Sigma_{u}^{+} \sim \Pi_{u}^{\prime} \sim \Delta_{u} .
\end{aligned}
$$

Similar observations have also been made by Foster and Michael (1999). In pNRQCD they emerge in a quite clear and straightforward way and one can explicitly write down the relevant operators. For higher excitations the expected degeneracies have been obtained by Bali and Pineda (2004). We discuss them further when comparing with lattice data in Sec. VI.D.

So far only the symmetries of pNRQCD at lowest order in the multipole expansion have been used. In fact one can go beyond that and predict the shape of the static energies by calculating the singlet and gluelump (static hybrid) correlators,

$$
\begin{gathered}
\left\langle\operatorname{vac}\left|H(\mathbf{R}, \mathbf{r}, T / 2) H^{\dagger}\left(\mathbf{R}^{\prime}, \mathbf{r}^{\prime},-T / 2\right)\right| \operatorname{vac}\right\rangle \\
\sim \delta^{3}\left(\mathbf{R}-\mathbf{R}^{\prime}\right) \delta^{3}\left(\mathbf{r}-\mathbf{r}^{\prime}\right) e^{-i T V_{H}(r)}, \\
\left\langle\operatorname{vac}\left|S(\mathbf{R}, \mathbf{r}, T / 2) S^{\dagger}\left(\mathbf{R}^{\prime}, \mathbf{r}^{\prime},-T / 2\right)\right| \operatorname{vac}\right\rangle \\
\sim \delta^{3}\left(\mathbf{R}-\mathbf{R}^{\prime}\right) \delta^{3}\left(\mathbf{r}-\mathbf{r}^{\prime}\right) e^{-i T V_{s}^{(0)}(r)},
\end{gathered}
$$

for large $T$. At lowest order in the multipole expansion the spectrum of the singlet state reads ${ }^{20}$

$$
E_{s}(r)=2 m+V_{s}^{(0)}(r)+\mathcal{O}\left(r^{2}\right) .
$$

For the static hybrids, the spectrum reads $\left[V_{H}=V_{o}^{(0)}(r)\right.$ $\left.+\Lambda_{H}\right]$

\footnotetext{
${ }^{20}$ By taking the arbitrary subtraction constant as twice the pole mass of a heavy quark in Eqs. (205) and (206), these equations become renormalon-free.
}

$$
E_{H}(r)=2 m+V_{o}^{(0)}(r)+\Lambda_{H}+\mathcal{O}\left(r^{2}\right),
$$

where

$$
\Lambda_{H} \equiv \lim _{T \rightarrow \infty} \frac{i}{T} \ln \left\langle H^{a}(T / 2) \phi_{a b}^{\mathrm{adj}}(T / 2,-T / 2) H^{b}(-T / 2)\right\rangle .
$$

Note that Eq. (207) allows us to relate the correlation length of some gluonic correlators to the behavior of the spectrum of the static hybrids at short distances. Note also that $\Lambda_{H}$ is the same for operators corresponding to states that are degenerate.

The potentials $V_{s}^{(0)}$ and $V_{o}^{(0)}$ can be computed within perturbation theory. One could then perform a detailed comparison with lattice data. We will see that in order to do so we have to deal first with the renormalon ambiguities as explained in Sec. V.

\section{The singlet static potential at short distances versus lattice}

In the last few years, lattice simulations (Bali et al., 1997; Necco and Sommer, 2002) have improved their predictions at short distances allowing very accurate comparisons between perturbation theory and lattice simulations. In order to perform this comparison, we cannot work in the on-shell scheme due to the presence of the renormalon, which destroys the convergence of the perturbative series. Therefore schemes were introduced to make the renormalon cancellation explicit. In the work of Recksiegel and Sumino (2002) and Sumino (2002) the renormalon cancellation is achieved order by order in $\alpha_{s}$ by expanding both $m$ and $V_{s}^{(0)}$ in terms of the same $\alpha_{s}(\nu)$. A potential problem of this method is the appearance of large logarithms in the mass expansion. In the results of Necco and Sommer (2001), lattice data were shown to agree with perturbation theory at short distances if the force was used instead of the potential. It was shown by Pineda (2003b) that this is equivalent to working in a renormalon-free scheme, and a first quantitative comparison of the (quenched) lattice data with the RS potential $V_{s, \mathrm{RS}}^{(0)}(r)$ was done (see also Lee, 2003b). This analysis allowed us to put quantitative bounds on nonperturbative effects at short distances and, in particular, it ruled out a linear potential with slope $\sigma$ $=0.21 \mathrm{GeV}^{2}$ at short distances (see also Pineda, 2004). It also showed that today lattice data are precise enough to be sensitive to three-loop perturbation theory (see Fig. 14). Overall, up to distances of around $(0.4-0.5) r_{0}$, perturbation theory is convergent with small errors and agrees with lattice data in all of the previous analyses. For larger distances the analysis done by Pineda (2003b) shows agreement with the lattice data (within errors) up to distances of $\sim 0.8 r_{0}$ if large logarithms are resummed. In the paper by Recksiegel and Sumino (2003) it was argued that by fine-tuning the renormalization scale, agreement with lattice data could be reached (within errors) up to $3 r_{0}$. Nevertheless, for such large distances, the use of perturbation theory is quite doubtful. There- 


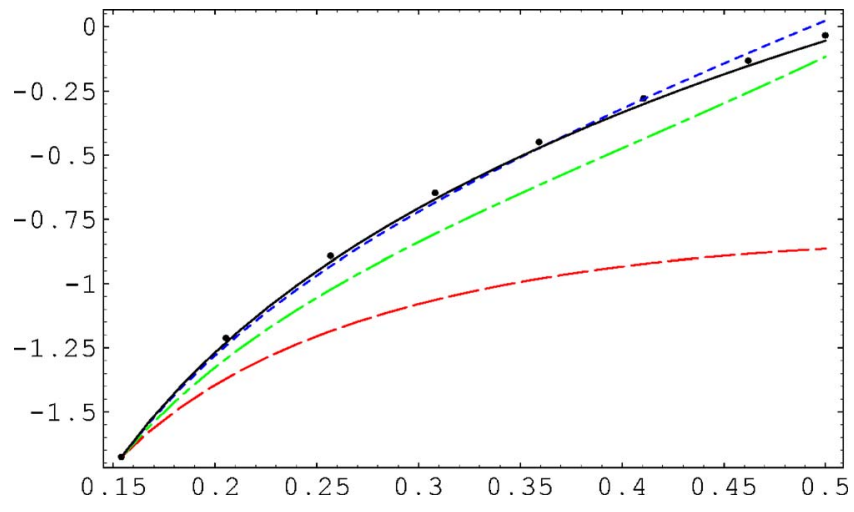

FIG. 14. (Color online) Plot of $r_{0}\left[V_{\mathrm{RS}}(r)-V_{\mathrm{RS}}\left(r^{\prime}\right)+E_{\text {latt. }}\left(r^{\prime}\right)\right]$ vs $r$ at tree (dashed line), one-loop (dash-dotted line), two-loop (dotted line), and three-loop level (estimate) plus the renormalization-group (RG) expression for the ultrasoft (US) logarithms (solid line) compared with the lattice simulations $E_{\text {latt. }}(r)$ (Necco and Sommer, 2002). For the scale of $\alpha_{s}(\nu)$, we set $\nu=1 / r$. Further, $\nu_{f}=\nu_{u s}=2.5 r_{0}^{-1}, \Lambda_{\overline{M S}}=0.602 r_{0}^{-1}$ (Capitani et al., 1999), and $r^{\prime}=0.15399 r_{0}$. From Pineda, 2003b.

fore further studies are needed to see whether this agreement is purely accidental or a theoretical explanation can be given.

\section{Gluelumps versus lattice}

We compare the predictions of pNRQCD for the static hybrids in the weak-coupling regime with lattice data. We first explore at which distances the expected degeneracies start to be fulfilled and whether the gluelump mass and hybrid potential splittings agree with each other (see Fig. 13). On a qualitative level the shortdistance data are consistent with the expected degeneracies. In any case, at best one can imagine perturbation theory to be valid for the left-most two data points. With the exception of the $\Pi_{u}, \Pi_{u}^{\prime}$, and $\Phi_{u}$ potentials there are also no clear signs of the onset of the short-distance $1 / r$ behavior with a positive coefficient as expected from perturbation theory. Furthermore, most of the gaps within multiplets of hybrid potentials, which at leading order depend on the size of the nonperturbative $r^{2}$ term, are still quite significant, even at $r=0.4 r_{0}$; for instance, the difference between the $\Sigma_{u}^{-}$and $\Pi_{u}$ potentials at this distance is about $0.28 r_{0}^{-1} \approx 110 \mathrm{MeV}$.

From the above considerations it is clear that for a more quantitative study one needs lattice data at shorter distances. These have been provided by Bali and Pineda (2004) for the lowest two gluonic excitations, $\Pi_{u}$ and $\Sigma_{u}^{-}$. We display their differences in the continuum limit in Fig. 15. We see how these approach zero at small $r$, as expected from the short-distance expansion. pNRQCD predicts that the next effects should be of $\mathcal{O}\left(r^{2}\right)$ (and renormalon-free). The lattice data are fitted rather well by a $\Delta E_{\Pi_{u}-\Sigma_{g}^{+}}=A_{\Pi_{u}-\Sigma_{u}^{-}} r^{2}$ ansatz for short distances, with slope (see Fig. 15)

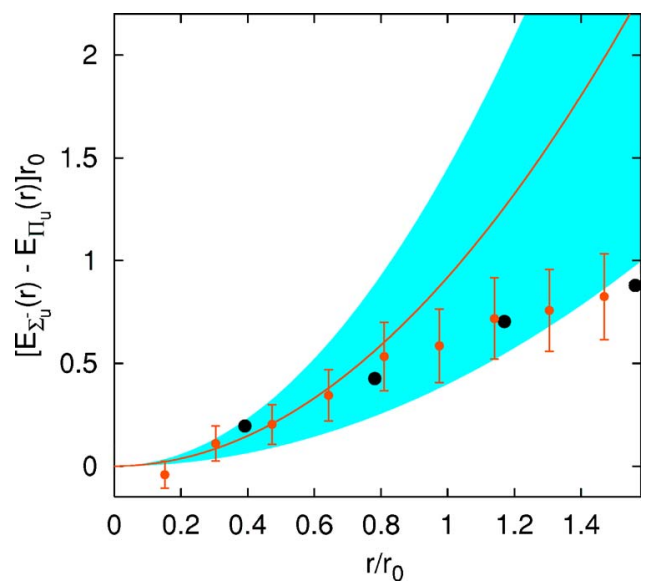

FIG. 15. (Color online) Splitting between the $\Sigma_{u}^{-}$and $\Pi_{u}$ potentials extrapolated to the continuum limit and comparison with a quadratic fit to the $r \leqq 0.5 r_{0}$ data points $\left(r_{0}^{-1} \approx 0.4 \mathrm{GeV}\right)$. The big circles correspond to the data obtained by Juge et al. (2003). The errors in this case are smaller than the symbols. The smaller circles correspond to the data obtained by Bali and Pineda, 2004. From Bali and Pineda, 2004.

$$
A_{\Pi_{u}-\Sigma_{u}^{-}}=0.92_{-0.52}^{+0.53} r_{0}^{-3},
$$

where the error is purely statistical (lattice), the systematic error being negligible. We remark that within the framework of static pNRQCD and to second order in the multipole expansion, one can relate the slope $A_{\Pi_{u}-\Sigma_{u}^{-}}$ to gluonic correlators of QCD.

One can go beyond these analyses and use lattice data plus the knowledge of the (perturbative) octet potential to obtain numerical values for gluelump masses in a particular scheme. However, analogous to the situation with the static singlet potential, the convergence of the octet potential perturbative series is poor. The solution to this problem comes again from working in a RS scheme properly generalized to the hybrid case. The hybrid energy is

$E_{H}(r)=2 m_{\mathrm{RS}}\left(\nu_{f}\right)+V_{o, \mathrm{RS}}\left(r ; \nu_{f}\right)+\Lambda_{H}^{\mathrm{RS}}\left(\nu_{f}\right)+\mathcal{O}\left(r^{2}\right)$.

In the RS scheme the octet potential is

$$
V_{o, \mathrm{RS}}\left(\nu_{f}\right)=V_{o}-\delta V_{o, \mathrm{RS}}=\sum_{n=0}^{\infty} V_{o, n}^{\mathrm{RS}} \alpha_{s}^{n+1},
$$

where

$\delta V_{o, \mathrm{RS}}=\sum_{n=1}^{\infty} N_{V_{o}} \nu_{f}\left(\frac{\beta_{0}}{2 \pi}\right)^{n} \alpha_{s}^{n+1}\left(\nu_{f}\right) \sum_{k=0}^{\infty} c_{k} \frac{\Gamma(n+1+b-k)}{\Gamma(1+b-k)}$.

This specifies the gluelump mass

$$
\Lambda_{H}^{\mathrm{RS}}\left(\nu_{f}\right)=\Lambda_{H}-\delta \Lambda_{\mathrm{RS}}\left(\nu_{f}\right),
$$

where 
TABLE II. Absolute values for the gluelump masses in the continuum limit in the RS scheme at $\nu_{f}=2.5 r_{0}^{-1} \approx 1 \mathrm{GeV}$, in $r_{0}$ units and in $\mathrm{GeV}$. Note that an additional uncertainty of about $10 \%$ should be added to the last column to account for the quenched approximation. We also display examples of creation operators $H$ for these states. The curly braces denote complete symmetrization of the indices. From Bali and Pineda, 2004.

\begin{tabular}{cccc}
\hline \hline$J^{P C}$ & $H$ & $\Lambda_{H}^{\mathrm{RS}} r_{0}$ & $\Lambda_{H}^{\mathrm{RS}} / \mathrm{GeV}$ \\
\hline $1^{+-}$ & $B_{i}$ & $2.25(39)$ & $0.87(15)$ \\
$1^{--}$ & $E_{i}$ & $3.18(41)$ & $1.25(16)$ \\
$2^{--}$ & $D_{\{i} B_{j\}}$ & $3.69(42)$ & $1.45(17)$ \\
$2^{+-}$ & $D_{\{i} E_{j\}}$ & $4.72(48)$ & $1.86(19)$ \\
$3^{+-}$ & $D_{\{i} D_{j} B_{k\}}$ & $4.72(45)$ & $1.86(18)$ \\
$0^{++}$ & $\mathbf{B}^{2}$ & $5.02(46)$ & $1.98(18)$ \\
$4^{--}$ & $D_{\{i} D_{j} D_{k} B_{l\}}$ & $5.41(46)$ & $2.13(18)$ \\
$1^{-+}$ & $(\mathbf{B} \wedge \mathbf{E})_{i}$ & $5.45(51)$ & $2.15(20)$ \\
\hline \hline
\end{tabular}

$$
\begin{aligned}
\delta \Lambda_{\mathrm{RS}}\left(\nu_{f}\right)= & \sum_{n=1}^{\infty} N_{\Lambda} \nu_{f}\left(\frac{\beta_{0}}{2 \pi}\right)^{n} \alpha_{s}^{n+1}\left(\nu_{f}\right) \\
& \times \sum_{k=0}^{\infty} c_{k} \frac{\Gamma(n+1+b-k)}{\Gamma(1+b-k)} .
\end{aligned}
$$

Note that factorization requires

$$
2 N_{m}+N_{V_{o}}+N_{\Lambda}=0
$$

$N_{m}$ is already known and $N_{V_{0}}$ can also be obtained approximately from low orders in perturbation theory following the same procedure as in Sec. V.A. One now has a convergent series in perturbation theory and can obtain absolute values for the masses of the gluelumps, in particular, for the lowest gluelump using the splitting of the $\Sigma_{g}^{+}$and the $\Pi_{u}$ potential. Then using the lattice data obtained by Foster and Michael (1999), it is possible to calculate the absolute values for the masses of all gluelump excitations in a given scheme (in this case, the RS scheme). The results are summarized in Table II. For a comparison with other determinations, see the work of Bali and Pineda (2004).

\section{POTENTIAL NRQCD. THE STRONG-COUPLING REGIME}

In this section we discuss pNRQCD when $\Lambda_{\mathrm{QCD}} \gg E$. In Sec. III we have called this situation the strongcoupling regime of $\mathrm{pNRQCD}$, and some general features of the physical picture have already been discussed. Since the EFT does not tell us anything about the nonperturbative dynamics of QCD, we have to rely on some assumptions in order to identify the relevant degrees of freedom. The assumptions will be minimal, supported by general considerations and lattice data, but clearly we are on less solid ground here than in the weak-coupling regime.

\section{A. Degrees of freedom}

If we consider the case without light quarks, the physical states made by a heavy quark and antiquark are heavy-quarkonium states or hybrids or both of them in the presence of glueballs. Quenched lattice data show that the static energy of the lowest state is separated by a gap of order $\Lambda_{\mathrm{QCD}}$ from the higher ones. This feature is preserved in going to unquenched simulations (see Fig. 4). We assume that this feature is also preserved in the dynamical case of heavy quarks with finite masses. This leads to identifying the heavy quarkonium with the solution of the Schrödinger equation on which the static potential corresponds to the ground-state static energy.

Once light fermions have been incorporated, however, new gauge-invariant states appear besides the heavy quarkonium, hybrids, and glueballs. First, we have states with no heavy-quark content. Due to chiral symmetry, there is a mass gap of $\mathcal{O}\left(\Lambda_{\mathrm{QCD}}\right)$ between the Goldstone bosons, which are massless in the chiral limit, and the rest of the spectrum. Therefore the Goldstone bosons are US degrees of freedom, while the rest of the spectrum is integrated out at the scale $\Lambda_{\mathrm{QCD}}$. Besides these, we also have bound states made of one heavy quark and light quarks. In practice, we are considering the $Q \bar{q}-\bar{Q} q$ system. The energy of this system is, according to the HQET counting rules (Neubert, 1994), $m_{Q \bar{q}}$ $+m_{\bar{Q} q}=2 m+2 \bar{\Lambda}$. Therefore since $\bar{\Lambda} \sim \Lambda_{\mathrm{QCD}}$, we assume that these states are also integrated out at the scale $\Lambda_{\mathrm{QCD}}$. This cannot be done for heavy-quarkonium states near threshold, since in this case there is no mass gap between the heavy quarkonium and the creation of a $Q \bar{q}-\bar{Q} q$ pair. Thus if we want to study the heavy quarkonium near threshold, we should include these degrees of freedom in the spectrum [for a model-dependent approach to this situation see, for instance, Eichten et al. (1978)]. We assume here that the heavy-quarkonium 
states under construction are safely far from threshold. ${ }^{21}$

In summary, the pNRQCD degrees of freedom in the regime $\Lambda_{\mathrm{QCD}} \gg E$ for quarkonium states far from threshold are a singlet field $S$, describing the heavyquarkonium state, and Goldstone boson fields. In the following, we shall not consider the Goldstone boson fields. If one switches off the light fermions, only the singlet survives and pNRQCD reduces to a pure twoparticle nonrelativistic quantum-mechanical system, usually referred to as a pure potential model.

\section{B. Power counting}

The structure of the pNRQCD Lagrangian under the above conditions is very simple: it is just a bilinear in the singlet field. Therefore establishing the power counting means to estimate the size of the terms multiplying the bilinear.

The soft scale $|\mathbf{p}|$ must be assigned to $-i \boldsymbol{\nabla}_{\mathbf{r}}$ and $1 / r$, the US scale $E \sim \mathbf{p}^{2} / m$ to the time derivatives $i \partial_{0}$ and $V_{s}^{(0)}$. This last condition follows from the consistency of the theory that requires the virial theorem be met. In other words, all the terms in the Schrödinger equation,

$$
i \partial_{0} \phi=E \phi=\left(\mathbf{p}^{2} / m+V_{s}^{(0)}\right) \phi
$$

must count the same. Note that the normalization condition of the wave function $\left[\int d^{3} \mathbf{r}|\phi(\mathbf{r})|^{2}=1\right]$ sets $|\phi|^{2}$ $\sim|\mathbf{p}|^{3}$. In general, the $1 / m$ corrections to the potential (real and imaginary) will be a combination of $\alpha_{s}$ calculated at different scales, derivatives with respect to the relative coordinate $-i \boldsymbol{\nabla}_{\mathbf{r}}, 1 / r$, and expectation values of the fields of the light degrees of freedom. The quantities $m$ and $\alpha_{s}(m)$ are inherited from the hard matching and have well-known values, in particular $\alpha_{s}(m) \ll 1$. The strong-coupling constant also appears evaluated at the scales $\sqrt{m \Lambda_{\mathrm{QCD}}}, 1 / r, \Lambda_{\mathrm{QCD}}$, and $E$. At the scale $\sqrt{m \Lambda_{\mathrm{QCD}}}$, which appears in loop calculations (see below), $\alpha_{s}(m)$ $\ll \alpha_{s}\left(\sqrt{m \Lambda_{\mathrm{QCD}}}\right) \ll 1$ since $\sqrt{m \Lambda_{\mathrm{QCD}}} \gg \Lambda_{\mathrm{QCD}}$. At the scales $\Lambda_{\mathrm{QCD}}$ and $E, \alpha_{s}\left(\Lambda_{\mathrm{QCD}}\right) \sim 1$ and $\alpha_{s}(E) \sim 1$ by definition of the strong-coupling regime. If $|\mathbf{p}| \sim \Lambda_{\mathrm{QCD}}$, then $\alpha_{s}(1 / r)$ $\sim 1$. If $|\mathbf{p}| \gg \Lambda_{\mathrm{QCD}} \gg E$, then $\alpha_{s}\left(\sqrt{m \Lambda_{\mathrm{QCD}}}\right) \ll \alpha_{s}(1 / r) \ll 1$. In the situation $|\mathbf{p}| \sim \Lambda_{\mathrm{OCD}}$, the expectation values of the fields of the light degrees of freedom depend on $\mathbf{r}$ and $\Lambda_{\mathrm{QCD}}$, while in the situation $|\mathbf{p}| \gg \Lambda_{\mathrm{QCD}} \gg E$, the $1 / r$ $\sim|\mathbf{p}|$ dependence factorizes and the expectation values of the fields of the light degrees of freedom depend only on $\Lambda_{\mathrm{QCD}} \cdot{ }^{22}$ In both cases their natural counting is $\Lambda_{\mathrm{QCD}}$ to the power of their dimension.

\footnotetext{
${ }^{21}$ One may think of relaxing this condition in the large $-N_{c}$ limit, where the mixing between the heavy quarkonium and the $Q \bar{q}-\bar{Q} q$ is suppressed by powers of $1 / N_{c}$.

${ }^{22}$ This is certainly so for states with low principal quantum number $n$. For higher excitations one should keep in mind that p and $1 / r$ could scale differently with $n$.
}

\section{Lagrangian and symmetries}

The pNRQCD Lagrangian (without Goldstone bosons) is given by

$$
L_{\mathrm{pNRQCD}}=\int d^{3} \mathbf{R} \int d^{3} \mathbf{r} S^{\dagger}\left[i \partial_{0}-h_{s}\left(\mathbf{x}_{1}, \mathbf{x}_{2}, \mathbf{p}_{1}, \mathbf{p}_{2}, \mathbf{S}_{1}, \mathbf{S}_{2}\right)\right] S,
$$

where

$$
\begin{aligned}
h_{s}\left(\mathbf{x}_{1}, \mathbf{x}_{2}, \mathbf{p}_{1}, \mathbf{p}_{2}, \mathbf{S}_{1}, \mathbf{S}_{2}\right)= & \frac{\mathbf{p}_{1}^{2}}{2 m_{1}}+\frac{\mathbf{p}_{2}^{2}}{2 m_{2}} \\
& +V_{s}\left(\mathbf{x}_{1}, \mathbf{x}_{2}, \mathbf{p}_{1}, \mathbf{p}_{2}, \mathbf{S}_{1}, \mathbf{S}_{2}\right),
\end{aligned}
$$

$\mathbf{p}_{j}=-i \nabla_{\mathbf{x}_{j}}, \mathbf{r}=\mathbf{x}_{1}-\mathbf{x}_{2}, \mathbf{R}=\left(\mathbf{x}_{1}+\mathbf{x}_{2}\right) / 2$, and $\mathbf{S}_{j}$ is the spin operator of particle $j$. In the following, unless stated differently, we assume $m_{1} \neq m_{2}$. However, we shall not exploit a possible hierarchy between the two masses, which for our purposes are of the same order $\sim m \gg \Lambda_{\mathrm{QCD}}$. The potential $V_{s}$ contains a real and an imaginary part, with the real part responsible for the binding and the imaginary part for the decay width of the heavy-quarkonium state. The imaginary part of $V_{s}$ comes from the matching coefficients of the four-fermion operators of NRQCD. The potential $V_{s}$ is, in general, a nonperturbative quantity, even if to some degree it may be obtained with perturbation theory, as the matching coefficients of NRQCD, or in general any contribution coming from scales larger than $\Lambda_{\mathrm{QCD}}$. It is the aim of the matching procedure, which we discuss in the following sections, to provide the factorization formulas and the exact expressions for the nonperturbative pieces. These may eventually be calculated on the lattice or in QCD vacuum models, which will be the subject of Sec. VII.G.

The symmetries of the singlet field are those already discussed for the pNRQCD Lagrangian in the weakcoupling regime. In particular, the potential and kinetic energies satisfy the Poincaré invariance constraints (85) and (86) (for the singlet potential). Note that Poincaré invariance may also constrain the natural power counting discussed in Sec. VII.B.

\section{Matching: analytic and nonanalytic mass terms}

Despite the fact that the strong-coupling Lagrangian (216) looks quite simple, the matching procedure that leads to it may be complicated. This is due to the fact that we have to integrate out, and therefore to make explicit, all the degrees of freedom (or momentum regions) that appear in the range from the hard to the US scale within a nonperturbative environment.

Since we are also integrating out $\Lambda_{\mathrm{QCD}}$, new momentum regions (apart from $\Lambda_{\mathrm{QCD}}$ itself) that do not appear in the weak-coupling matching show up. Let us consider, for instance, the diagram of Fig. 16. Suppose that the incoming (outcoming) particle is an off-shell particle of energy $\sim \Lambda_{\mathrm{QCD}}$ and three-momentum $p(q) \sim m v$ [for in- 


\begin{tabular}{|c|c|c|c|c|}
\hline \multirow{5}{*}{$(E, \mathrm{p})$} & $T$ & & T & \multirow{5}{*}{$(E, \mathrm{q})$} \\
\hline & 1 & $(E, \xi)$ & I & \\
\hline & I & & I & \\
\hline & I & & I & \\
\hline & 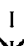 & & I & \\
\hline
\end{tabular}

FIG. 16. The incoming energy $E$ is of order $\Lambda_{\mathrm{QCD}}, p$, and $q$ of order $m v$. The vertex describes the interaction with an external potential $V$.

stance, an on-shell particle that just emitted (absorbed) a soft gluon of energy $\Lambda_{\mathrm{QCD}}$. The diagram corresponds to the integral

$$
\int \frac{d^{3} \boldsymbol{\xi}}{(2 \pi)^{3}} V(\mathbf{p}-\boldsymbol{\xi}) \frac{1}{E-\boldsymbol{\xi}^{2} / m+i \boldsymbol{\epsilon}} V(\boldsymbol{\xi}-\mathbf{q}) .
$$

This integral also receives a contribution from the threemomentum region $\xi \sim \sqrt{m E} \sim \sqrt{m \Lambda_{\mathrm{QCD}}}$. Since $\sqrt{m \Lambda_{\mathrm{QCD}}}$ $\gg \Lambda_{\mathrm{QCD}}$, the potential is perturbative, and since $\sqrt{m \Lambda_{\mathrm{QCD}}} \gg p, q$, we may expand in $p$ and $q$ and the integral effectively reduces to

$$
\alpha_{s}^{2} \int \frac{d^{3} \xi}{(2 \pi)^{3}} \frac{1}{\xi^{4}} \frac{1}{E-\xi^{2} / m+i \epsilon} \sim \alpha_{s}^{2} \frac{1}{\Lambda_{\mathrm{QCD}}} \frac{1}{\sqrt{m \Lambda_{\mathrm{QCD}}}},
$$

where $\alpha_{s}$ is calculated at the (perturbative) scale $\sqrt{m \Lambda_{\mathrm{QCD}}}$. From the above example we may draw the following conclusions. First, in the strong-coupling regime new degrees of freedom show up in loops, namely, quark-antiquark pairs with relative three-momentum of order $\sqrt{m \Lambda_{\mathrm{QCD}}}$ and on-shell energy of order $\Lambda_{\mathrm{QCD}}$. Since the scale $\sqrt{m \Lambda_{\mathrm{QCD}}} \gg|\mathbf{p}|$ for $\Lambda_{\mathrm{OCD}} \gg E$, this is the largest scale below $m$ and thus the first to be integrated out. The only reason this otherwise dominant contribution to the potential is suppressed is that it appears only in loops. Second, since we expand in the external momenta, which are small compared to $\sqrt{m \Lambda_{\mathrm{QCD}}}$, the effective interaction that arises is local. Third, this kind of contribution is nonanalytic in $m$.

It is convenient to split the potential (imaginary and real parts) into a part that gets contributions only from scales that are analytic in the mass, $V^{1 / m}$, and another, $V^{1 / \sqrt{m}}$, that contains any contribution coming from the scale $\sqrt{m \Lambda_{\mathrm{QCD}}}$ :

$$
V_{s}=V^{1 / m}+V^{1 / \sqrt{m}}
$$

We often refer to $V^{1 / \sqrt{m}}$ as the part of the potential that is nonanalytic in $1 / \mathrm{m}$. This is only true at $\mathrm{LO}$, which is, however, the order at which we work here. The matching for the $V^{1 / m}$ part may be performed in a strict $1 / \mathrm{m}$ expansion. The matching for the $V^{1 / \sqrt{m}}$ part may be done by integrating out quark-antiquark pairs with relative three-momenta of order $\sqrt{m \Lambda_{\mathrm{QCD}}}$.

We next discuss the matching procedures for $V^{1 / m}$ and $V^{1 / \sqrt{m}}$ when $|\mathbf{p}| \sim \Lambda_{\mathrm{QCD}}$ and $|\mathbf{p}| \gg \Lambda_{\mathrm{QCD}} \gg E$. We will first consider the case $|\mathbf{p}| \sim \Lambda_{\mathrm{QCD}}$ in Sec. VII.E. The potential will be a function of $\mathbf{r}$ and $\Lambda_{\mathrm{QCD}}$. This is the most general case. The particular case $|\mathbf{p}| \gg \Lambda_{\mathrm{QCD}} \gg E$ may be derived by factorizing the potential in a high-energy part dependent on $1 / r \sim|\mathbf{p}|$ and a low-energy part dependent on $\Lambda_{\mathrm{QCD}}$. In this case, however, it is more practical and consistent with the general philosophy of the EFT to achieve factorization directly by integrating out the scales $|\mathbf{p}|$ and $\Lambda_{\mathrm{OCD}}$ in two different steps of the matching procedure. We consider this situation in Sec. VII.F. We note here that local terms that appear when $|\mathbf{p}|$ $\sim \Lambda_{\mathrm{QCD}}$ are already factorized and therefore will be reproduced (up to field redefinitions) when $|\mathbf{p}| \gg \Lambda_{\mathrm{QCD}}$ $\gg E$. This describes the imaginary part of the potential, which comes from the four-fermion contact terms of the NRQCD Lagrangian and the part of the potential that is nonanalytic in $1 / \mathrm{m}$.

Finally, we mention that soft light fermions will not be explicitly considered in the matching computation. If we want to incorporate them, the procedure would be analogous. One would have to consider the matrix elements and Wilson loops with dynamical light fermions incorporated and new terms appearing in the energies at $\mathcal{O}\left(1 / \mathrm{m}^{2}\right)$ due to operators involving light fermions that appear in the NRQCD Lagrangian at $\mathcal{O}\left(1 / \mathrm{m}^{2}\right)$ and the Gauss law.

\section{E. Matching for $|\mathbf{p}| \sim \Lambda_{\mathrm{QCD}}$}

In Sec. VI.A, we discussed the static limit of NRQCD. The spectrum consists of the static energies $E_{0}^{(0)} \ll E_{1}^{(0)}$ $\ll \cdots$. We assume a gap of order $\Lambda_{\mathrm{QCD}}$ between $E_{0}^{(0)}$ and the higher excitations. pNRQCD is, by definition, the EFT that describes the lowest excitation of the NRQCD spectrum. From Eq. (216) it follows that pNRQCD in the static limit consists of a singlet field $S$ with static energy $V^{(0)}$. Since the static energy is an observable, the matching condition in the static limit is

$$
E_{0}^{(0)}(r)=V^{(0)}(r) .
$$

Note that the left-hand side is a quantity defined in NRQCD, while the right-hand side is a matching coefficient of pNRQCD.

We may think of generalizing the matching condition (221) to the nonstatic case. Similar to what was done in Sec. VI.A, we introduce the normalized eigenstates, $\left|\underline{\mathrm{n}} ; \mathbf{x}_{1}, \mathbf{x}_{2}\right\rangle$, and eigenvalues, $E_{n}\left(\mathbf{x}_{1}, \mathbf{x}_{2} ; \mathbf{p}_{1}, \mathbf{p}_{2}\right)$, of the full NRQCD Hamiltonian $H$. They satisfy the equations

$$
\begin{aligned}
& H\left|\underline{\mathrm{n}} ; \mathbf{x}_{1}, \mathbf{x}_{2}\right\rangle= \int d^{3} x_{1}^{\prime} d^{3} x_{2}^{\prime}\left|\underline{\mathrm{n}} ; \mathbf{x}_{1}^{\prime}, \mathbf{x}_{2}^{\prime}\right\rangle \\
& \times E_{n}\left(\mathbf{x}_{1}^{\prime}, \mathbf{x}_{2}^{\prime}, \mathbf{p}_{1}^{\prime}, \mathbf{p}_{2}^{\prime}, \mathbf{S}_{1}, \mathbf{S}_{2}\right) \\
& \times \delta^{(3)}\left(\mathbf{x}_{1}^{\prime}-\mathbf{x}_{1}\right) \delta^{(3)}\left(\mathbf{x}_{2}^{\prime}-\mathbf{x}_{2}\right) \\
&\left\langle\underline{\mathrm{m}} ; \mathbf{x}_{1}, \mathbf{x}_{2} \mid \underline{\mathrm{n}} ; \mathbf{y}_{1}, \mathbf{y}_{2}\right\rangle=\delta_{n m} \delta^{(3)}\left(\mathbf{x}_{1}-\mathbf{y}_{1}\right) \delta^{(3)}\left(\mathbf{x}_{2}-\mathbf{y}_{2}\right)
\end{aligned}
$$

where the states are labeled with the positions $\mathbf{x}_{1}$ and $\mathbf{x}_{2}$ of the static solution even if the position operator does not commute with $H$ beyond the static limit. The eigenvalues $E_{n}$ are, in general, functions of the momentum 
and spin operators and therefore should be understood as operators as well. We assume a gap of order $\Lambda_{\mathrm{QCD}}$ between (the levels of) $E_{0}$ and (the levels of) $E_{n}$ for $n$ $>0$. Under this circumstance, and arguing as in the static case above, it follows that the matching condition is

$$
E_{0}\left(\mathbf{x}_{1}, \mathbf{x}_{2}, \mathbf{p}_{1}, \mathbf{p}_{2}, \mathbf{S}_{1}, \mathbf{S}_{2}\right)=h_{s}\left(\mathbf{x}_{1}, \mathbf{x}_{2}, \mathbf{p}_{1}, \mathbf{p}_{2}, \mathbf{S}_{1}, \mathbf{S}_{2}\right)
$$

Again, this equation expresses the (real and imaginary parts of the) pNRQCD Hamiltonian in terms of a quantity

$$
\begin{aligned}
& E_{0}\left(\mathbf{x}_{1}, \mathbf{x}_{2}, \mathbf{p}_{1}, \mathbf{p}_{2}, \mathbf{S}_{1}, \mathbf{S}_{2}\right) \delta^{(3)}\left(\mathbf{x}_{1}-\mathbf{y}_{1}\right) \delta^{(3)}\left(\mathbf{x}_{2}-\mathbf{y}_{2}\right) \\
& \quad=\left\langle\underline{0} ; \mathbf{x}_{1}, \mathbf{x}_{2}|H| \underline{0} ; \mathbf{y}_{1}, \mathbf{y}_{2}\right\rangle,
\end{aligned}
$$

defined in NRQCD. The aim of the matching is to calculate this quantity. As discussed above, it will contain a part that is analytic in $1 / \mathrm{m}$ and another that is not.

\section{Matching of the analytic terms: quantum-mechanical matching}

The analytic part of $E_{0}$ can be calculated, by definition, in a strict $1 / \mathrm{m}$ expansion. The idea is to split the NRQCD Hamiltonian as

$$
H=H^{(0)}+H_{I},
$$

where $H^{(0)}$ is the static Hamiltonian, whose eigenstates and eigenvalues have been discussed in Sec. VI.A, and

$$
H_{I}=\frac{H^{(1,0)}}{m_{1}}+\frac{H^{(0,1)}}{m_{2}}+\frac{H^{(2,0)}}{m_{1}^{2}}+\frac{H^{(0,2)}}{m_{2}^{2}}+\frac{H^{(1,1)}}{m_{1} m_{2}}+\cdots
$$

is the sum of all higher-order terms in the $1 / \mathrm{m}$ expansion of the NRQCD Hamiltonian. Then solve Eq. (222) using quantum-mechanical perturbation theory around the static solution. Calculated in this way, the eigenstates (and eigenvalues) of Eq. (222) result as expansions in powers of $1 / \mathrm{m}$ :

$$
\begin{aligned}
\left|\underline{\mathbf{n}} ; \mathbf{x}_{1}, \mathbf{x}_{2}\right\rangle= & \left|\underline{\mathbf{n}} ; \mathbf{x}_{1}, \mathbf{x}_{2}\right\rangle^{(0)}+\frac{1}{m_{1}}\left|\underline{\mathbf{n}} ; \mathbf{x}_{1}, \mathbf{x}_{2}\right\rangle^{(1,0)} \\
& +\frac{1}{m_{2}}\left|\underline{\underline{\mathbf{n}}} ; \mathbf{x}_{1}, \mathbf{x}_{2}\right\rangle^{(0,1)}+\frac{1}{m_{1}^{2}}\left|\underline{\underline{n}} ; \mathbf{x}_{1}, \mathbf{x}_{2}\right\rangle^{(2,0)} \\
& +\frac{1}{m_{2}^{2}}\left|\underline{\underline{\mathbf{n}}} ; \mathbf{x}_{1}, \mathbf{x}_{2}\right\rangle^{(0,2)}+\frac{1}{m_{1} m_{2}}\left|\underline{\mathbf{n}} ; \mathbf{x}_{1}, \mathbf{x}_{2}\right\rangle^{(1,1)} \\
& +\cdots
\end{aligned}
$$

A complete derivation can be found in the original literature (Brambilla, Pineda, et al., 2001; Pineda and Vairo, 2001; Brambilla, Eiras, et al., 2003). ${ }^{23}$ Here we only make a few remarks. First, the expressions for $\left|\underline{n} ; \mathbf{x}_{1}, \mathbf{x}_{2}\right\rangle^{(1,0)}$ and $\left|\underline{n} ; \mathbf{x}_{1}, \mathbf{x}_{2}\right\rangle^{(2,0)}$ look similar to the wellknown formulas of time-independent perturbation

\footnotetext{
${ }^{23}$ A similar approach has been used by Szczepaniak and Swanson (1997) in order to derive, from the QCD Hamiltonian in the Coulomb gauge, the spin-dependent part of the potential up to $\mathcal{O}\left(1 / m^{2}\right)$.
}

theory in quantum mechanics, the only difference being the fact that the energies $E_{n}^{(0)}$ depend on spatial coordinates and that the matrix elements of $H^{(1,0)}$ and $H^{(2,0)}$ are operators in the quantum-mechanical sense. Second, as usually done in quantum mechanics, we have set the relative phase between $\left|\underline{n} ; \mathbf{x}_{1}, \mathbf{x}_{2}\right\rangle$ and $\left|\underline{n} ; \mathbf{x}_{1}, \mathbf{x}_{2}\right\rangle^{(0)}$ to 1 in Eq. (228). This choice is arbitrary. The freedom of choice reflects the fact that the eigenvalues and eigenstates solution of Eq. (222) are defined up to a unitary transformation $e^{i O_{n}}$ (with $\left.O_{n}^{\dagger}=O_{n}\right)$ :

$$
\begin{aligned}
& \left|\underline{\underline{n}}, \mathbf{x}_{1}, \mathbf{x}_{2}\right\rangle \rightarrow \int d^{3} \mathbf{x}_{1}^{\prime} d^{3} \mathbf{x}_{2}^{\prime}\left|\underline{\underline{n}}, \mathbf{x}_{1}^{\prime}, \mathbf{x}_{2}^{\prime}\right\rangle e^{i O_{n}\left(\mathbf{x}_{1}^{\prime}, \mathbf{x}_{2}^{\prime}, \mathbf{p}_{1}^{\prime}, \mathbf{p}_{2}^{\prime}, \mathbf{s}_{1}, \mathbf{s}_{2}\right)} \\
& \times \delta^{(3)}\left(\mathbf{x}_{1}^{\prime}-\mathbf{x}_{1}\right) \delta^{(3)}\left(\mathbf{x}_{2}^{\prime}-\mathbf{x}_{2}\right), \\
& E_{n}\left(\mathbf{x}_{1}, \mathbf{x}_{2}, \mathbf{p}_{1}, \mathbf{p}_{2}, \mathbf{S}_{1}, \mathbf{S}_{2}\right) \\
& \rightarrow \int d^{3} \mathbf{x}_{1}^{\prime} d^{3} \mathbf{x}_{2}^{\prime} e^{i O_{n}\left(\mathbf{x}_{1}^{\prime}, \mathbf{x}_{2}^{\prime}, \mathbf{p}_{1}^{\prime}, \mathbf{p}_{2}^{\prime}, \mathbf{S}_{1}, \mathbf{S}_{2}\right)} E_{n}\left(\mathbf{x}_{1}^{\prime}, \mathbf{x}_{2}^{\prime}, \mathbf{p}_{1}^{\prime}, \mathbf{p}_{2}^{\prime}, \mathbf{S}_{1}, \mathbf{S}_{2}\right) \\
& \times e^{-i O_{n}\left(\mathbf{x}_{1}^{\prime}, \mathbf{x}_{2}^{\prime}, \mathbf{p}_{1}^{\prime}, \mathbf{p}_{2}^{\prime}, \mathbf{S}_{1}, \mathbf{S}_{2}\right)} \delta^{(3)}\left(\mathbf{x}_{1}^{\prime}-\mathbf{x}_{1}\right) \delta^{(3)}\left(\mathbf{x}_{2}^{\prime}-\mathbf{x}_{2}\right) .
\end{aligned}
$$

Our choice preserves the power counting and allows us to obtain rather compact expressions for the potentials. Third, from the expression for the state $\left|\underline{0} ; \mathbf{x}_{1}, \mathbf{x}_{2}\right\rangle$, the expression for the energy $E_{0}$ may be derived straightforwardly, order by order in $1 / m$, from Eq. (225). Finally, the matching condition (224) gives the pNRQCD Hamiltonian.

In order to transform the quantum-mechanical expressions into expressions that only contain expectation values of gluon fields, the following steps are necessary.

(i) The first step is to integrate out the fermion fields. They appear in the matrix elements of $H^{(1)}$ and $H^{(2)}$ either in the states [see Eq. (198)] or in the Hamiltonian itself as two- or four-fermion interaction terms. In the first case, we have, for instance,

$$
\begin{aligned}
{ }^{(0)}\left\langle\underline{\underline{n}} ; \mathbf{x}_{1}, \mathbf{x}_{2}\left|\int d^{3} \boldsymbol{\xi} \psi^{\dagger}(\boldsymbol{\xi}) O(\xi) \psi(\xi)\right| \underline{\mathrm{m}} ; \mathbf{y}_{1}, \mathbf{y}_{2}\right\rangle^{(0)} \\
={ }^{\left({ }^{(0)}\right.}\left\langle n ; \mathbf{x}_{1}, \mathbf{x}_{2}\left|O\left(\mathbf{x}_{1}\right)\right| m ; \mathbf{x}_{1}, \mathbf{x}_{2}\right\rangle^{(0)} \delta^{(3)}\left(\mathbf{x}_{1}-\mathbf{y}_{1}\right) \\
\quad \times \delta^{(3)}\left(\mathbf{x}_{2}-\mathbf{y}_{2}\right),
\end{aligned}
$$

in the second case

$$
\begin{aligned}
{ }^{(0)}\left\langle\underline{\mathrm{n}} ; \mathbf{x}_{1}, \mathbf{x}_{2}\right| \int d^{3} \boldsymbol{\xi} \psi^{\dagger}(\boldsymbol{\xi}) O_{A}(\boldsymbol{\xi}) \psi(\boldsymbol{\xi}) \chi_{c}^{\dagger}(\boldsymbol{\xi}) O_{B}(\boldsymbol{\xi}) \chi_{c}(\boldsymbol{\xi}) \\
\quad \times\left|\underline{\mathrm{m}} ; \mathbf{y}_{1}, \mathbf{y}_{2}\right\rangle^{(0)} \\
=\delta^{(3)}\left(\mathbf{x}_{1}-\mathbf{x}_{2}\right)^{(0)}\left\langle n ; \mathbf{x}_{1}, \mathbf{x}_{2}\right| O_{A}\left(\mathbf{x}_{1}\right) O_{B}\left(\mathbf{x}_{2}\right) \\
\quad \times\left|m ; \mathbf{x}_{1}, \mathbf{x}_{2}\right\rangle^{(0)} \delta^{(3)}\left(\mathbf{x}_{1}-\mathbf{y}_{1}\right) \delta^{(3)}\left(\mathbf{x}_{2}-\mathbf{y}_{2}\right)
\end{aligned}
$$

where $O, O_{A}$, and $O_{B}$ are combinations of gluon fields. In the last case, the interaction is local $\left[\sim \delta^{(3)}(\mathbf{r})\right]$. At this stage, the expressions only contain matrix elements of gluon fields on the pure gluonic states $\left|n ; \mathbf{x}_{1}, \mathbf{x}_{2}\right\rangle^{(0)}$ $\equiv|n\rangle^{(0)}$. At this point, it is also possible to use the Gauss law (197). It allows us to write all the terms of the type $[\mathbf{D}, g \mathbf{E}]$ in terms of $\delta^{(3)}(\mathbf{r})$ times some color matrices, up 
to terms proportional to $\delta^{(3)}(\mathbf{0})$ that vanish in DR. We shall assume this regularization scheme from now on.

(ii) Further simplifications may be achieved using the identities $\left[F_{1,2} \equiv F\left(\mathbf{x}_{1,2}\right)\right]$

${ }^{(0)}\left\langle n\left|\mathbf{D}_{1}\right| n\right\rangle^{(0)}=\nabla_{1}, \quad{ }^{(0)}\left\langle n\left|\mathbf{D}_{c 2}\right| n\right\rangle^{(0)}=\nabla_{2}$,

${ }^{(0)}\left\langle n\left|\mathbf{D}_{1}\right| j\right\rangle^{(0)}=\frac{{ }^{(0)}\left\langle n\left|g \mathbf{E}_{1}\right| j\right\rangle^{(0)}}{E_{n}^{(0)}-E_{j}^{(0)}}$,

${ }^{(0)}\left\langle n\left|\mathbf{D}_{c 2}\right| j\right\rangle^{(0)}=-\frac{{ }^{(0)}\left\langle n\left|g \mathbf{E}_{2}^{T}\right| j\right\rangle^{(0)}}{E_{n}^{(0)}-E_{j}^{(0)}} \quad \forall n \neq j$,

${ }^{(0)}\left\langle n\left|g \mathbf{E}_{1}\right| n\right\rangle^{(0)}=-\left(\boldsymbol{\nabla}_{1} E_{n}^{(0)}\right), \quad{ }^{(0)}\left\langle n\left|g \mathbf{E}_{2}^{T}\right| n\right\rangle^{(0)}=\left(\nabla_{2} E_{n}^{(0)}\right)$,

where $\mathbf{D}_{c}$ is the charge conjugate of $\mathbf{D}$. The first equality follows from symmetry considerations, the second and the third may be derived from ${ }^{(0)}\left\langle n\left|\left[H^{(0)}, \mathbf{D}\right]\right| j\right\rangle^{(0)}$ $=E_{n}^{(0)}{ }^{(0)}\langle n|\mathbf{D}| j\rangle^{(0)}{ }^{(0)}\langle n|\mathbf{D}| j\rangle^{(0)} E_{j}^{(0)}$ and the canonical commutation relations.

(iii) The last step consists in rewriting the quantummechanical expressions in terms of Wilson-loop amplitudes. We proceed by considering an interpolating state (in the Heisenberg representation) that has a nonvanishing overlap with the ground state:

$$
\left.\psi^{\dagger}\left(\mathbf{x}_{1}\right) \phi\left(\mathbf{x}_{1}, \mathbf{x}_{2}\right) \chi_{c}^{\dagger}\left(\mathbf{x}_{2}\right) \mid \text { vac }\right\rangle
$$

where $\phi$ makes the above state overlap with the ground state $\left|\underline{0} ; \mathbf{x}_{1}, \mathbf{x}_{2}\right\rangle^{(0)}$. We use here the popular choice (107), which assumes that the ground state has the $\Sigma_{g}^{+}$quantum numbers. We also define $\phi(\mathbf{y}, \mathbf{x} ; t=0) \equiv \phi(\mathbf{y}, \mathbf{x})$. Then we have

$$
\psi^{\dagger}\left(\mathbf{x}_{1}\right) \phi\left(\mathbf{x}_{1}, \mathbf{x}_{2}\right) \chi_{c}^{\dagger}\left(\mathbf{x}_{2}\right)|\mathrm{vac}\rangle=\sum_{n} a_{n}\left(\mathbf{x}_{1}, \mathbf{x}_{2}\right)\left|\underline{\mathrm{n}} ; \mathbf{x}_{1}, \mathbf{x}_{2}\right\rangle^{(0)},
$$

or, without fermion fields,

$$
\phi\left(\mathbf{x}_{1}, \mathbf{x}_{2}\right)|\mathrm{vac}\rangle=\sum_{n} a_{n}\left(\mathbf{x}_{1}, \mathbf{x}_{2}\right)\left|n ; \mathbf{x}_{1}, \mathbf{x}_{2}\right\rangle^{(0)},
$$

with $a_{0} \neq 0$. At this point, we define the Wilson-loop average $\langle\cdots\rangle_{\square} \equiv\left\langle\cdots W_{\square}\right\rangle$. The gauge fields are, in general, localized on the static quark lines of the Wilson loop. Therefore $\langle\cdots\rangle_{\square}$ is gauge invariant. Inserting the identity operator $\Sigma|n\rangle^{(0)}{ }^{(0)}\langle n|$ into the Wilson-loop averages, from Eq. (238) it follows that

$$
\begin{aligned}
& \left\langle W_{\square}\right\rangle=\sum_{n} e^{-i E_{n}^{(0)} T_{W}}\left|a_{n}\right|^{2}, \\
& \left\langle F^{(1)}\left(t_{1}\right) \cdots F^{(n)}\left(t_{n}\right)\right\rangle_{\square} \\
& =\sum_{n, m, s_{1}, \ldots, s_{n-1}} a_{n}^{*} a_{m}{ }^{(0)}\left\langle n\left|F^{(1)}\right| s_{1}\right\rangle^{(0)} \ldots{ }^{(0)}\left\langle s_{n-1}\left|F^{(n)}\right| m\right\rangle^{(0)}
\end{aligned}
$$

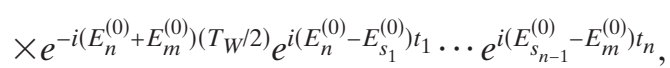

$$
\begin{aligned}
& \left\langle\left\langle F^{(1)}\left(t_{1}\right) \cdots F^{(n)}\left(t_{n}\right)\right\rangle\right\rangle \\
& \equiv \lim _{T_{W \rightarrow \infty}} \frac{\left\langle F^{(1)}\left(t_{1}\right) \cdots F^{(n)}\left(t_{n}\right)\right\rangle_{\square}}{\left\langle W_{\square}\right\rangle}(0) \\
& =\sum_{s_{1}, \ldots, s_{n-1}}{ }^{(0)}\left\langle 0\left|F^{(1)}\right| s_{1}\right\rangle^{(0)} \ldots{ }^{(0)}\left\langle s_{n-1}\left|F^{(n)}\right| 0\right\rangle^{(0)} \\
& \times e^{i\left(E_{0}^{(0)}-E_{s_{1}}^{(0)}\right) t_{1}} \cdots e^{i\left(E_{s_{n-1}}^{(0)}-E_{0}^{(0)}\right) t_{n}},
\end{aligned}
$$

where $T_{W} / 2 \geqslant t_{1} \geqslant t_{2} \geqslant \cdots \geqslant t_{n} \geqslant-T_{W} / 2$ and $F^{(n)}$ are gluon fields localized on the static Wilson loop. All the quantum-mechanical expressions obtained at the end of step (ii) may be expressed as combinations of

$$
\int_{0}^{\infty} d t_{1} \cdots \int_{0}^{t_{n-1}} d t_{n} t_{1}^{j_{1}} \cdots t_{n}^{j_{n}}\left\langle\left\langle F^{(1)}\left(t_{1}\right) \cdots F^{(n)}\left(t_{n}\right)\right\rangle\right\rangle_{c},
$$

where $\langle\langle\cdots\rangle\rangle_{c}$ stands for the connected part of $\langle\langle\cdots\rangle\rangle$.

\section{Matching of the analytic terms: the real pNRQCD potential}

We give here and in the following section the explicit formulas for the part of the pNRQCD potential that is analytic in $1 / \mathrm{m}$. For the real part, we give formulas up to (and including) order $1 / \mathrm{m}^{2}$, for the imaginary part up to (and including) order $1 / \mathrm{m}^{4}$. The formulas are given in four dimensions. Divergences have been regularized, if necessary, in DR. We have explicitly used the Gauss-law constraint (197). Note that we would need to generalize these formulas to $d$ dimensions to work in a MS-like scheme and consistently use the same scheme used for renormalizing the NRQCD matching coefficients.

Up to (and including) order $1 / \mathrm{m}^{2}$, the real part of the potential $V^{1 / m}$ may be written as in Eq. (63) and the $1 / m^{2}$ potentials may be decomposed in terms of their momentum and spin content as in Eqs. (65)-(71). The different pieces are given by Brambilla, Pineda, et al. (2001); Pineda and Vairo (2001):

$$
\begin{aligned}
& V^{(0)}(r)=\lim _{T_{W} \rightarrow \infty} \frac{i}{T_{W}} \ln \left\langle W_{\square}\right\rangle, \\
& V^{(1,0)}(r)=-\frac{1}{2} \int_{0}^{\infty} d t t\left\langle\left\langle g \mathbf{E}_{1}(t) \cdot g \mathbf{E}_{1}(0)\right\rangle\right\rangle_{c}, \\
& V^{(0,1)}(r)=V^{(1,0)}(r),
\end{aligned}
$$




$$
\begin{aligned}
& V_{\mathbf{p}^{2}}^{(2,0)}(r)=\frac{i}{2} \hat{\mathbf{r}}^{i} \hat{\mathbf{r}}^{j} \int_{0}^{\infty} d t t^{2}\left\langle\left\langle g \mathbf{E}_{1}^{i}(t) g \mathbf{E}_{1}^{j}(0)\right\rangle\right\rangle_{c}, \\
& V_{\mathbf{L}^{2}}^{(2,0)}(r)=\frac{i}{4}\left(\delta^{i j}-3 \hat{\mathbf{r}^{i}} \hat{\mathbf{r}}^{j}\right) \int_{0}^{\infty} d t t^{2}\left\langle\left\langle g \mathbf{E}_{1}^{i}(t) g \mathbf{E}_{1}^{j}(0)\right\rangle\right\rangle_{c} \\
& V_{r}^{(2,0)}(r)=\frac{\pi C_{F} \alpha_{s} c_{D}^{(1)}}{2} \delta^{(3)}(\mathbf{r})-\frac{i c_{F}^{(1) 2}}{4} \int_{0}^{\infty} d t\left\langle\left\langle g \mathbf{B}_{1}(t) \cdot g \mathbf{B}_{1}(0)\right\rangle\right\rangle_{c}+\frac{1}{2}\left(\nabla_{\mathbf{r}}^{2} V_{\mathbf{p}^{2}}^{(2,0)}\right)-\frac{i}{2} \int_{0}^{\infty} d t_{1} \int_{0}^{t_{1}} d t_{2} \int_{0}^{t_{2}} d t_{3}\left(t_{2}-t_{3}\right)^{2} \\
& \times\left\langle\left\langle g \mathbf{E}_{1}\left(t_{1}\right) \cdot g \mathbf{E}_{1}\left(t_{2}\right) g \mathbf{E}_{1}\left(t_{3}\right) \cdot g \mathbf{E}_{1}(0)\right\rangle\right\rangle_{c}+\frac{1}{2}\left(\nabla_{\mathbf{r}}^{i} \int_{0}^{\infty} d t_{1} \int_{0}^{t_{1}} d t_{2}\left(t_{1}-t_{2}\right)^{2}\left\langle\left\langle g \mathbf{E}_{1}^{i}\left(t_{1}\right) g \mathbf{E}_{1}\left(t_{2}\right) \cdot g \mathbf{E}_{1}(0)\right\rangle\right\rangle_{c}\right) \\
& -\frac{i}{2}\left(\nabla_{\mathbf{r}}^{i} V^{(0)}\right) \int_{0}^{\infty} d t_{1} \int_{0}^{t_{1}} d t_{2}\left(t_{1}-t_{2}\right)^{3}\left\langle\left\langle g \mathbf{E}_{1}^{i}\left(t_{1}\right) g \mathbf{E}_{1}\left(t_{2}\right) \cdot g \mathbf{E}_{1}(0)\right\rangle\right\rangle_{c}+\frac{1}{4}\left(\nabla_{\mathbf{r}}^{i} \int_{0}^{\infty} d t t^{3}\left\langle\left\langle g \mathbf{E}_{1}^{i}(t) g \mathbf{E}_{1}^{j}(0)\right\rangle\right\rangle_{c}\left(\nabla_{\mathbf{r}}^{j} V^{(0)}\right)\right) \\
& -\frac{i}{12} \int_{0}^{\infty} d t t^{4}\left\langle\left\langle g \mathbf{E}_{1}^{i}(t) g \mathbf{E}_{1}^{j}(0)\right\rangle\right\rangle_{c}\left(\nabla_{\mathbf{r}}^{i} V^{(0)}\right)\left(\nabla_{\mathbf{r}}^{j} V^{(0)}\right)-\frac{c_{1}^{g(1)}}{4} f_{a b c} \int d^{3} \mathbf{x}\left\langle\left\langle g G_{\mu \nu}^{a}(x) G_{\mu \alpha}^{b}(x) G_{\nu \alpha}^{c}(x)\right\rangle\right\rangle, \\
& V_{\mathbf{p}^{2}}^{(0,2)}(r)=V_{\mathbf{p}^{2}}^{(2,0)}(r), \quad V_{\mathbf{L}^{2}}^{(0,2)}(r)=V_{\mathbf{L}^{2}}^{(2,0)}(r), \quad V_{r}^{(0,2)}(r)=V_{r}^{(2,0)}\left(r ; m_{2} \leftrightarrow m_{1}\right), \\
& V_{\mathbf{p}^{2}}^{(1,1)}(r)=i \hat{\mathbf{r}} \hat{i} \hat{\mathbf{r}}^{j} \int_{0}^{\infty} d t t^{2}\left\langle\left\langle g \mathbf{E}_{1}^{i}(t) g \mathbf{E}_{2}^{j}(0)\right\rangle\right\rangle_{c} \\
& V_{\mathbf{L}^{2}}^{(1,1)}(r)=i \frac{\delta^{i j}-3 \hat{\mathbf{r}}^{i} \hat{\mathbf{r}}^{j}}{2} \int_{0}^{\infty} d t t^{2}\left\langle\left\langle g \mathbf{E}_{1}^{i}(t) g \mathbf{E}_{2}^{j}(0)\right\rangle\right\rangle_{c} \\
& V_{r}^{(1,1)}(r)=-\frac{1}{2}\left(\nabla_{\mathbf{r}}^{2} V_{\mathbf{p}^{2}}^{(1,1)}\right) \delta^{(3)}(\mathbf{r})-i \int_{0}^{\infty} d t_{1} \int_{0}^{t_{1}} d t_{2} \int_{0}^{t_{2}} d t_{3}\left(t_{2}-t_{3}\right)^{2}\left\langle\left\langle g \mathbf{E}_{1}\left(t_{1}\right) \cdot g \mathbf{E}_{1}\left(t_{2}\right) g \mathbf{E}_{2}\left(t_{3}\right) \cdot g \mathbf{E}_{2}(0)\right\rangle\right\rangle_{c} \\
& +\frac{1}{2}\left(\nabla_{\mathbf{r}}^{i} \int_{0}^{\infty} d t_{1} \int_{0}^{t_{1}} d t_{2}\left(t_{1}-t_{2}\right)^{2}\left\langle\left\langle g \mathbf{E}_{1}^{i}\left(t_{1}\right) g \mathbf{E}_{2}\left(t_{2}\right) \cdot g \mathbf{E}_{2}(0)\right\rangle\right\rangle_{c}\right) \\
& +\frac{1}{2}\left(\nabla_{\mathbf{r}}^{i} \int_{0}^{\infty} d t_{1} \int_{0}^{t_{1}} d t_{2}\left(t_{1}-t_{2}\right)^{2}\left\langle\left\langle g \mathbf{E}_{2}^{i}\left(t_{1}\right) g \mathbf{E}_{1}\left(t_{2}\right) \cdot g \mathbf{E}_{1}(0)\right\rangle\right\rangle_{c}\right) \\
& -\frac{i}{2}\left(\nabla_{\mathbf{r}}^{i} V^{(0)}\right) \int_{0}^{\infty} d t_{1} \int_{0}^{t_{1}} d t_{2}\left(t_{1}-t_{2}\right)^{3}\left\langle\left\langle g \mathbf{E}_{1}^{i}\left(t_{1}\right) g \mathbf{E}_{2}\left(t_{2}\right) \cdot g \mathbf{E}_{2}(0)\right\rangle\right\rangle_{c}-\frac{i}{2}\left(\nabla_{\mathbf{r}}^{i} V^{(0)}\right) \int_{0}^{\infty} d t_{1} \int_{0}^{t_{1}} d t_{2}\left(t_{1}\right. \\
& \left.-t_{2}\right)^{3}\left\langle\left\langle g \mathbf{E}_{2}^{i}\left(t_{1}\right) g \mathbf{E}_{1}\left(t_{2}\right) \cdot g \mathbf{E}_{1}(0)\right\rangle\right\rangle_{c}+\frac{1}{4}\left(\nabla_{\mathbf{r}}^{i} \int_{0}^{\infty} d t t^{3}\left\{\left\langle\left\langle g \mathbf{E}_{1}^{i}(t) g \mathbf{E}_{2}^{j}(0)\right\rangle\right\rangle_{c}+\left\langle\left\langle g \mathbf{E}_{2}^{i}(t) g \mathbf{E}_{1}^{j}(0)\right\rangle\right\rangle_{c}\right\}\left(\nabla_{\mathbf{r}}^{j} V^{(0)}\right)\right) \\
& -\frac{i}{6} \int_{0}^{\infty} d t t^{4}\left\langle\left\langle g \mathbf{E}_{1}^{i}(t) g \mathbf{E}_{2}^{j}(0)\right\rangle\right\rangle_{c}\left(\nabla_{\mathbf{r}}^{i} V^{(0)}\right)\left(\nabla_{\mathbf{r}}^{j} V^{(0)}\right),-\frac{C_{A}}{2}\left[\operatorname{Re} f_{1}\left({ }^{1} S_{0}\right)+3 \operatorname{Re} f_{1}\left({ }^{3} S_{1}\right)\right] \delta^{(3)}(\mathbf{r}), \\
& V_{L S}^{(2,0)}(r)=-\frac{c_{F}^{(1)}}{r^{2}} i \mathbf{r} \cdot \int_{0}^{\infty} d t t\left\langle\left\langle g \mathbf{B}_{1}(t) \times g \mathbf{E}_{1}(0)\right\rangle\right\rangle+\frac{c_{S}^{(1)}}{2 r^{2}} \mathbf{r} \cdot\left(\nabla_{\mathbf{r}} V^{(0)}\right), \\
& V_{L S}^{(0,2)}(r)=V_{L S}^{(2,0)}\left(r ; m_{2} \leftrightarrow m_{1}\right), \\
& V_{L_{2} S_{1}}^{(1,1)}(r)=-\frac{c_{F}^{(1)}}{r^{2}} i \mathbf{r} \cdot \int_{0}^{\infty} d t t\left\langle\left\langle g \mathbf{B}_{1}(t) \times g \mathbf{E}_{2}(0)\right\rangle\right\rangle, \\
& V_{L_{1} S_{2}}^{(1,1)}(r)=V_{L_{2} S_{1}}^{(1,1)}\left(r ; m_{1} \leftrightarrow m_{2}\right),
\end{aligned}
$$




$$
\begin{aligned}
& V_{S^{2}}^{(1,1)}(r)=\frac{2 c_{F}^{(1)} c_{F}^{(2)}}{3} i \int_{0}^{\infty} d t\left\langle\left\langle g \mathbf{B}_{1}(t) \cdot g \mathbf{B}_{2}(0)\right\rangle\right\rangle+2 C_{A}\left[\operatorname{Re} f_{1}\left({ }^{1} S_{0}\right)-\operatorname{Re} f_{1}\left({ }^{3} S_{1}\right)\right] \delta^{(3)}(\mathbf{r}), \\
& V_{\mathbf{S}_{12}}^{(1,1)}(r)=\frac{c_{F}^{(1)} c_{F}^{(2)}}{4} i \hat{\mathbf{r}}^{i} \hat{\mathbf{r}}^{j} \int_{0}^{\infty} d t\left[\left\langle\left\langle g \mathbf{B}_{1}^{i}(t) g \mathbf{B}_{2}^{j}(0)\right\rangle\right\rangle-\frac{\delta^{i j}}{3}\left\langle\left\langle g \mathbf{B}_{1}(t) \cdot g \mathbf{B}_{2}(0)\right\rangle\right\rangle\right]
\end{aligned}
$$

Equations (245), (249), (254), and (256) follow from invariance under simultaneous charge conjugation and $m_{1} \leftrightarrow m_{2}$ exchange.

Equation (243) is the well-known formula that gives the static potential in terms of the static Wilson loop (Susskind, 1977; Brown and Weisberger, 1979). In the weak-coupling case, this formula requires corrections from US degrees of freedom (in that case, US gluons). Here, by assumption, we do not have other US degrees of freedom besides the heavy-quarkonium singlet field, hence there are no corrections. Once Goldstone bosons are taken into account, their contribution will eventually correct Eq. (243). With respect to the power counting, for dimensional reasons $V^{(0)}$ would count as $|\mathbf{p}|$. In Sec. VII.B we have argued, however, that the NR dynamics constrains $V^{(0)}$ to count as $E$. The extra suppression of order $E /|\mathbf{p}| \sim v$ has to arise on dynamical grounds. In the perturbative case, it originates from the factor $\alpha_{s} \sim v$ in the potential. In the nonperturbative case little can be said and some other mechanism must be responsible.

Equation (244) gives the $1 / \mathrm{m}$ corrections to the static potential. They were first calculated by Brambilla, Pineda, et al. (2001). In accordance with the power counting of Sec. VII.B, these corrections are of the or$\operatorname{der} \Lambda_{\mathrm{QCD}}^{2} / m \sim E$ when $|\mathbf{p}| \sim \Lambda_{\mathrm{QCD}}$. Therefore they may, in principle, be as large as the static potential. In the weak-coupling regime, the first nonvanishing contribution to $V^{(1,0)}$ is of order $\alpha_{s}^{2}$ and gives $V^{(1,0)}(r)$ $=-C_{F} C_{A} \alpha_{s}^{2} / 4 r^{2}$, which is suppressed by $\alpha_{s}^{2}$ with respect to the static potential.

Equations (246), (247), (250), and (251) are momentum-dependent $1 / \mathrm{m}^{2}$ potentials. They were first derived using a quantum-mechanical path-integral approach by Barchielli et al. (1988). Equations (248) and (252) are momentum- and spin-independent $1 / \mathrm{m}^{2}$ potentials. Their calculation was first done by Pineda and Vairo (2001). Note that they are necessary in order to solve the ordering ambiguity that plagues the calculation of the momentum-dependent potentials. The momentum- and spin-independent $1 / \mathrm{m}^{2}$ potentials also depend on some of the matching coefficients of NRQCD. The last term of Eq. (248) comes from the $1 / \mathrm{m}^{2}$ corrections to the Yang-Mills Lagrangian of NRQCD. It is somehow different from the other terms since the fields are not localized on the Wilson-loop lines. Moreover, it exhibits a fictitious dependence on the time at which the operator insertion is made, which disappears in the limit $T_{W} \rightarrow \infty$. However, the term is not as peculiar as it may at first appear if we notice that $V^{(0)}$ could also be written in a similar way: $V^{(0)}$ $=\frac{1}{2} \int d^{3} \mathbf{x}\left\langle\left\langle\left(\boldsymbol{\Pi}^{a} \boldsymbol{\Pi}^{a}+\mathbf{B}^{a} \mathbf{B}^{a}\right)(x)\right\rangle\right\rangle$.

Equation (255) gives the spin-orbit, Eq. (257) the spinspin, and Eq. (258) the spin-tensor $1 / \mathrm{m}^{2}$ potential. These potentials were first derived, by Eichten and Feinberg (1981), in the approach that we discuss in Sec. VII.E.4 and rederived later by several authors in similar or different approaches, for instance, by Peskin (1983); Gromes (1984); Barchielli et al. (1988). None of the early derivations included the NRQCD matching coefficients, which were first included by Chen et al. (1995); see also Brambilla and Vairo (1999b). Pineda and Vairo (2001) corrected an error in the formula of the spin-orbit potential $V_{L_{2} S_{1}}^{(1,1)}$ that can be found in the original papers (Eichten and Feinberg, 1981; Gromes, 1984; Barchielli et al., 1988; Chen et al., 1995). For a detailed analysis and comments on this, see Brambilla, Gromes, and Vairo (2001) and Pineda and Vairo (2001).

In the $|\mathbf{p}| \sim \Lambda_{\mathrm{QCD}}$ regime, the leading terms contributing to the $1 / \mathrm{m}^{2}$ potentials are of the order $\Lambda_{\mathrm{QCD}}^{3} / m$. Not all the terms contribute, however, to the same order. Terms involving $\nabla_{\mathbf{r}} V^{(0)}$ have an extra $\mathcal{O}(v)$ suppression coming from the specific counting of $V^{(0)}$. Terms involving matching coefficients of NRQCD also have an expansion in $\alpha_{s}$. Since the matching coefficients of the four-fermion and of the pure Yang-Mills operators of NRQCD start at order $\alpha_{s}$, terms involving them are suppressed by a factor $\alpha_{s}$. In particular, if we consider the potentials with more terms, $V_{r}^{(2,0)}$ and $V_{r}^{(1,1)}$, only the terms in the first two and three lines listed in Eqs. (248) and (252), respectively, are expected to contribute at LO. In the weak-coupling regime, there is an extra $\alpha_{s}$ suppression coming from the $g^{2}$ in the Wilson-loop amplitudes and the $1 / m^{2}$ potentials give the familiar $m \alpha_{s}^{4}$ relativistic, fine, and hyperfine corrections to the perturbative spectrum.

The Poincaré invariance constraints (86) become in the present case with different masses

$$
\begin{aligned}
& V_{L S}^{(2,0)}(r)-V_{L_{2} S_{1}}^{(1,1)}(r)+\frac{V^{(0) \prime}(r)}{2 r}=0, \\
& V_{\mathbf{L}^{2}}^{(2,0)}(r)+V_{\mathbf{L}^{2}}^{(0,2)}(r)-V_{\mathbf{L}^{2}}^{(1,1)}(r)+\frac{r}{2} V^{(0) \prime}(r)=0,
\end{aligned}
$$




$$
\begin{aligned}
& -2\left[V_{\mathbf{p}^{2}}^{(2,0)}(r)+V_{\mathbf{p}^{2}}^{(0,2)}(r)\right]+2 V_{\mathbf{p}^{2}}^{(1,1)}(r)-V^{(0)}(r) \\
& +r V^{(0))}(r)=0 .
\end{aligned}
$$

These are general symmetry relations, independent of the dynamics. However, due to the potentials given above, they now impose specific relations among the Wilson-loop amplitudes and the matching coefficients of NRQCD, which can be tested independently. Taking at tree level the NRQCD matching coefficients, Eq. (259) was proved by Gromes (1984), and Eqs. (260) and (261) by Barchielli et al. (1990). A way to proceed is the following (Brambilla, Gromes, and Vairo, 2001). Consider a chromoelectric- or a chromomagnetic-field insertion in a static Wilson loop and then apply an infinitesimal Lorentz boost with velocity $\mathbf{v}$. The following identities hold:

$$
\begin{aligned}
& \left\langle\left\langle g \mathbf{B}\left(\mathbf{x}_{1}, t\right)\right\rangle\right\rangle^{\text {boosted }}+\left\langle\left\langle\left[\mathbf{v} \times g \mathbf{E}\left(\mathbf{x}_{1}, t\right)\right]\right\rangle\right\rangle^{\text {boosted }} \\
& \quad-\left\langle\left\langle g \mathbf{B}\left(\mathbf{x}_{1}, t\right)\right\rangle\right\rangle=0, \\
& \left\langle\left\langle i g \hat{\mathbf{v}} \cdot \mathbf{E}\left(\mathbf{x}_{1}, t\right)\right\rangle\right\rangle-\left\langle\left\langle i g \hat{\mathbf{v}} \cdot \mathbf{E}\left(\mathbf{x}_{1}, t\right)\right\rangle\right\rangle^{\text {boosted }}=0 .
\end{aligned}
$$

Expanding both equations at order $v$ and $v^{2}$, respectively, and considering that the difference between the boosted and the static Wilson loop corresponds to insertions of chromoelectric fields, we obtain from the first equation

$$
\begin{aligned}
& -i \int_{0}^{\infty} d t t\left[\left\langle\left\langle g \mathbf{B}\left(\mathbf{x}_{1}, t\right) \times g \mathbf{E}\left(\mathbf{x}_{1}, 0\right)\right\rangle\right\rangle-\left\langle\left\langle g \mathbf{B}\left(\mathbf{x}_{1}, t\right)\right.\right.\right. \\
& \left.\left.\left.\quad \times g \mathbf{E}\left(\mathbf{x}_{2}, 0\right)\right\rangle\right\rangle\right]+\hat{\mathbf{r}} V^{(0){ }^{\prime}}(r)=0
\end{aligned}
$$

and from the second equations (260) and (261). These relations have also been tested on the lattice, as we shall discuss in Sec. VII.G.

Finally, we emphasize that the freedom we noticed at the level of NRQCD to perform a unitary transformation of the states and energies, Eqs. (229) and (230), is obviously preserved at the level of pNRQCD. The effect of a unitary field redefinition $U$ of the singlet field is to transform $h_{s} \rightarrow U^{\dagger} h_{s} U$, where $h_{s}$ is the pNRQCD Hamiltonian. This means that no special physical meaning is associated with a single potential term, which may be reshuffled into another by means of a suitable unitary transformation. In other words, unlike physical observables, which are unambiguous, potentials depend on the specific scheme adopted. The potentials listed in Eqs. (243)-(258) are given in the scheme defined by Eq. (228), which fixes the relative phase to 1 between $\left|\underline{n} ; \mathbf{x}_{1}, \mathbf{x}_{2}\right\rangle$ and $\left|\underline{n} ; \mathbf{x}_{1}, \mathbf{x}_{2}\right\rangle^{(0)}$. We refer the reader to Brambilla, Pineda, et al. (2001); see also Brambilla, Gromes, and Vairo (2001) for more details.

\section{Matching of the analytic terms: the imaginary pNRQCD potential}

Let us consider heavy quarkonia made of a quark and an antiquark of the same flavor $\left(m_{1}=m_{2}=m\right)$. Annihila- tion processes happen in QCD at the scale of the mass $m$. Integrating them out in the matching from QCD to NRQCD gives rise to imaginary contributions to the four-fermion matching coefficients. Under the assumptions that led to Eq. (216), they are the only source of contribution to the imaginary pNRQCD Hamiltonian, which can be calculated in the same way as the real part. In practice, the calculation reduces to picking up from the right-hand side of Eq. (225) only contributions that involve four-fermion operators.

From the above general considerations, the imaginary part of the potential $V^{1 / m}$ is

$$
\operatorname{Im} V^{1 / m}=\frac{\operatorname{Im} V^{(2)}}{m^{2}}+\frac{\operatorname{Im} V^{(4)}}{m^{4}}+\cdots
$$

The functions $\operatorname{Im} V^{(2)}$ and $\operatorname{Im} V^{(4)}$ encode the information from the dimension- 6 and the dimension- 8 fourfermion operators of NRQCD, respectively. They will have the following structure:

$$
\begin{aligned}
& (\text { spin }) \times(\text { delta }) \times(\operatorname{Im} f) \\
& \quad \times(\text { nonperturbative matrix element }) .
\end{aligned}
$$

The first factor, which is one of the projectors (77)-(81), accounts for the spin structure. The second is a delta function or (for $\operatorname{Im} V^{(n>2)}$ ) consists of derivatives of delta functions due to the fact that the four-fermion operators are local. The third is the imaginary part of a fourfermion matching coefficient of NRQCD. Note that, in general, the potential may also depend on some real matching coefficients of NRQCD. Finally, the last term is a matrix element that contains all soft gluons integrated out from NRQCD. These matrix elements are Wilson amplitudes, similar to those that appear in the real part of the pNRQCD potentials, taken in the $r \rightarrow 0$ limit due to the delta function. In other words, they are nonlocal (in time) correlators of gluonic fields $F$ : $\left\langle F^{(1)}\left(t_{1}, \mathbf{0}\right) \phi\left(t_{1}, t_{2}\right) \cdots F^{(n)}\left(t_{n}, \mathbf{0}\right) \phi\left(t_{n}, t_{1}\right)\right\rangle$. In the following we omit the Wilson lines $\phi$ connecting the fields and the spatial location of the fields which are irrelevant. The correlators that show up at order $1 / \mathrm{m}^{2}$ and $1 / \mathrm{m}^{4}$ are encoded in the nonperturbative parameters $\mathcal{E}_{1}, \mathcal{E}_{3}, \mathcal{B}_{1}$, $\mathcal{E}_{3}^{(2, t)}$, and $\mathcal{E}_{3}^{(2, \mathrm{EM})}$, where

$$
\begin{gathered}
\mathcal{E}_{n} \equiv \frac{1}{N_{c}} \int_{0}^{\infty} d t t^{n}\langle g \mathbf{E}(t) \cdot g \mathbf{E}(0)\rangle, \\
\mathcal{B}_{n} \equiv \frac{1}{N_{c}} \int_{0}^{\infty} d t t^{n}\langle g \mathbf{B}(t) \cdot g \mathbf{B}(0)\rangle,
\end{gathered}
$$

and the definitions of $\mathcal{E}_{3}^{(2, t)}$ and $\mathcal{E}_{3}^{(2, \mathrm{EM})}$, which involve four chromoelectric fields, can be found in the article by Brambilla, Eiras, et al. (2003).

The explicit expression for $\operatorname{Im} V^{(2)}$ is equal to Eq. (75), while $\operatorname{Im} V^{(4)}$ is given by Brambilla, Eiras, et al. (2002, 2003): 


$$
\begin{aligned}
& \operatorname{Im} V^{(4)}=C_{A} \mathcal{T}_{S J}^{i j} \nabla_{\mathbf{r}}^{i} \delta^{(3)}(\mathbf{r}) \nabla_{\mathbf{r}}^{j}\left[\operatorname{Im} f_{1}\left({ }^{2 S+1} P_{J}\right)+\operatorname{Im} f_{\mathrm{EM}}\left({ }^{2 S+1} P_{J}\right)\right]+\frac{C_{A}}{2} \Omega_{S J}^{i j}\left\{\nabla_{\mathbf{r}}^{i} \nabla_{\mathbf{r}}^{j}+\frac{\delta_{i j}}{3} \mathcal{E}_{1}, \delta^{(3)}(\mathbf{r})\right\}\left[\operatorname{Im} g_{1}\left({ }^{2 S+1} S_{J}\right)\right. \\
& \left.+\operatorname{Im} g_{\mathrm{EM}}\left({ }^{2 S+1} S_{J}\right)\right]+\frac{T_{F}}{3} \mathcal{T}_{S J}^{i i} \delta^{(3)}(\mathbf{r}) \operatorname{Im} f_{8}\left({ }^{2 S+1} P_{J}\right) \mathcal{E}_{1}+\frac{T_{F}}{9} \nabla_{\mathbf{r}} \delta^{(3)}(\mathbf{r}) \nabla_{\mathbf{r}}\left\{4 \operatorname{Im} f_{8}\left({ }^{1} S_{0}\right)-2 \mathbf{S}^{2}\left[\operatorname{Im} f_{8}\left({ }^{1} S_{0}\right)\right.\right. \\
& \left.\left.-\operatorname{Im} f_{8}\left({ }^{3} S_{1}\right)\right]\right\} \mathcal{E}_{3}+2 T_{F} c_{F}^{2} \delta^{(3)}(\mathbf{r})\left(\operatorname{Im} f_{8}\left({ }^{3} S_{1}\right)+\frac{1}{6} \mathbf{S}^{2}\left[\operatorname{Im} f_{8}\left({ }^{1} S_{0}\right)-3 \operatorname{Im} f_{8}\left({ }^{3} S_{1}\right)\right]\right) \mathcal{B}_{1}+\frac{T_{F}}{3} \delta^{(3)}(\mathbf{r})\left\{4 \operatorname{Im} f_{8}\left({ }^{1} S_{0}\right)\right. \\
& \left.-2 \mathbf{S}^{2}\left[\operatorname{Im} f_{8}\left({ }^{1} S_{0}\right)-\operatorname{Im} f_{8}\left({ }^{3} S_{1}\right)\right]\right\} \mathcal{E}_{3}^{(2)}-\frac{C_{A}}{3} \delta^{(3)}(\mathbf{r})\left\{4 \operatorname{Im} f_{1}\left({ }^{1} S_{0}\right)-2 \mathbf{S}^{2}\left[\operatorname{Im} f_{1}\left({ }^{1} S_{0}\right)-\operatorname{Im} f_{1}\left({ }^{3} S_{1}\right)\right]\right\} \mathcal{E}_{3}^{(2, t)} \\
& -C_{A} \frac{2}{9}\left\{\boldsymbol{\nabla}_{\mathbf{r}}^{2}, \delta^{(3)}(\mathbf{r})\right\}\left(\operatorname{Im} f_{1}\left({ }^{1} S_{0}\right)+\operatorname{Im} f_{\mathrm{EM}}\left({ }^{1} S_{0}\right)+\frac{\mathbf{S}^{2}}{2}\left[\operatorname{Im} f_{1}\left({ }^{3} S_{1}\right)-\operatorname{Im} f_{1}\left({ }^{1} S_{0}\right)+\operatorname{Im} f_{\mathrm{EM}}\left({ }^{3} S_{1}\right)-\operatorname{Im} f_{\mathrm{EM}}\left({ }^{1} S_{0}\right)\right]\right) \mathcal{E}_{3} \\
& -2 C_{A} c_{F}^{2} \delta^{(3)}(\mathbf{r})\left(\operatorname{Im} f_{1}\left({ }^{1} S_{0}\right)+\operatorname{Im} f_{\mathrm{EM}}\left({ }^{1} S_{0}\right)+\frac{\mathbf{S}^{2}}{6}\left[\operatorname{Im} f_{1}\left({ }^{3} S_{1}\right)-3 \operatorname{Im} f_{1}\left({ }^{1} S_{0}\right)+\operatorname{Im} f_{\mathrm{EM}}\left({ }^{3} S_{1}\right)-3 \operatorname{Im} f_{\mathrm{EM}}\left({ }^{1} S_{0}\right)\right]\right) \mathcal{B}_{1} \\
& -\frac{C_{A}}{3} \delta^{(3)}(\mathbf{r})\left\{4 \operatorname{Im} f_{\mathrm{EM}}\left({ }^{1} S_{0}\right)-2 \mathbf{S}^{2}\left[\operatorname{Im} f_{\mathrm{EM}}\left({ }^{1} S_{0}\right)-\operatorname{Im} f_{\mathrm{EM}}\left({ }^{3} S_{1}\right)\right]\right\} \mathcal{E}_{3}^{(2, \mathrm{EM})} .
\end{aligned}
$$

Note that there are more terms in Eq. (268) than in Eq. (76) due to the nonperturbative counting. Similar to the real case, the quantities $\operatorname{Im} V^{(2)}, \operatorname{Im} V^{(4)}, \ldots$ are defined up to unitary transformations. A discussion and an explicit example may be found in the article of Brambilla, Eiras, et al. (2003).

\section{Matching of the analytic terms: direct matching of Wilson-loop amplitudes}

In the previous sections we performed the matching to pNRQCD first by deriving quantum-mechanical expressions, then by translating them into Wilson-loop amplitudes. Hence one may wonder whether it would be possible to directly perform the matching to Wilson-loop amplitudes. This is possible and simply requires applying to the strong-coupling regime the Wilson-loop matching used in Sec. IV.F for the weak-coupling regime. The only difference will be that no US corrections have to be subtracted from the Wilson-loop amplitudes in this case. It should be noted that historically the first derivation of some of the heavy-quarkonium potentials was done by direct computation of Wilson-loop amplitudes, namely, the static potential (Susskind, 1977; Brown and Weisberger, 1979) the $1 / \mathrm{m}^{2}$ spin-dependent potentials (Eichten and Feinberg, 1981; Gromes, 1984), and the $\mathbf{p}^{i} \mathbf{p}^{j} / m^{2}$ spin-independent potentials (Barchielli et al., 1988). We now (re)derive the heavy-quarkonium potential up to (and including) order $1 / m$ by directly matching Wilsonloop amplitudes to pNRQCD Green's functions (Brambilla, Pineda, et al., 2001).

Let us consider the following Green's function of NRQCD:

$$
\begin{aligned}
G_{\mathrm{NRQCD}}= & \langle\operatorname{vac}\rangle \chi_{c}\left(\mathbf{x}_{2}, T_{W} / 2\right) \phi\left(\mathbf{x}_{2}, \mathbf{x}_{1} ; T_{W} / 2\right) \psi\left(\mathbf{x}_{1}, T_{W} / 2\right) \\
& \times \psi^{\dagger}\left(\mathbf{y}_{1},-T_{W} / 2\right) \phi\left(\mathbf{y}_{1}, \mathbf{y}_{2} ;-T_{W} / 2\right) \\
& \times \chi_{c}^{\dagger}\left(\mathbf{y}_{2},-T_{W} / 2\right)|\mathrm{vac}\rangle .
\end{aligned}
$$

Expanding $G_{\mathrm{NROCD}}$ order by order in $1 / m, G_{\mathrm{NROCD}}$ $=G_{\mathrm{NRQCD}}^{(0)}+\left(1 / m_{1}\right) G_{\mathrm{NRQCD}}^{(1,0)}+\left(1 / m_{2}\right) G_{\mathrm{NRQCD}}^{(0,1)}+\cdots, \quad$ and integrating out the fermion fields we obtain

$$
\begin{aligned}
& G_{\mathrm{NRQCD}}^{(0)}=\left\langle W_{\square}\right\rangle \delta^{(3)}\left(\mathbf{x}_{1}-\mathbf{y}_{1}\right) \delta^{(3)}\left(\mathbf{x}_{2}-\mathbf{y}_{2}\right), \\
& G_{\mathrm{NRQCD}}^{(1,0)}=\frac{i}{2} \int_{-T_{W} / 2}^{T_{W} / 2} d t\left\langle\mathbf{D}_{1}^{2}(t)\right\rangle_{\square} \delta^{(3)}\left(\mathbf{x}_{1}-\mathbf{y}_{1}\right) \delta^{(3)}\left(\mathbf{x}_{2}-\mathbf{y}_{2}\right) .
\end{aligned}
$$

For simplicity we shall not display here and in the following the analogous formulas for $G_{\mathrm{NROCD}}^{(0,1)}$. From time reversal it follows that $\left\langle\mathbf{B}_{1}(t)\right\rangle_{\square}=-\left\langle\mathbf{B}_{1}(-t)\right\rangle_{\square}$, which eliminates the spin-dependent term in Eq. (271). After some algebra it follows that

$$
\begin{aligned}
G_{\mathrm{NRQCD}}^{(1,0)}= & \frac{i}{2}\left\{\frac{T_{W}}{2} \nabla_{\mathbf{x}_{1}}^{2}\left\langle W_{\square}\right\rangle+\frac{T_{W}}{2}\left\langle W_{\square}\right\rangle \nabla_{\mathbf{x}_{1}}^{2}\right. \\
& +T_{W}\left\langle\mathbf{O}_{f}\left(T_{W} / 2\right) \cdot \mathbf{O}_{i}\left(-T_{W} / 2\right)\right\rangle_{\square} \\
& +i g \int_{-T_{W} / 2}^{T_{W} / 2} d t\left(\frac{T_{W}}{2}-t\right)\left\langle\mathbf{O}_{f}\left(T_{W} / 2\right) \cdot \mathbf{E}(t)\right\rangle_{\square} \\
& -i g \int_{-T_{W} / 2}^{T_{W} / 2} d t\left(\frac{T_{W}}{2}+t\right)\left\langle\mathbf{E}(t) \cdot \mathbf{O}_{i}\left(-T_{W} / 2\right)\right\rangle_{\square} \\
& +\frac{g^{2}}{2} \int_{-T_{W} / 2}^{T_{W} / 2} d t \int_{-T_{W} / 2}^{T_{W} / 2} d t^{\prime}\left|t-t^{\prime}\right| \\
& \left.\times\left\langle\mathbf{E}(t) \cdot \mathbf{E}\left(t^{\prime}\right)\right\rangle_{\square}\right\} \delta^{(3)}\left(\mathbf{x}_{1}-\mathbf{y}_{1}\right) \delta^{(3)}\left(\mathbf{x}_{2}-\mathbf{y}_{2}\right),
\end{aligned}
$$

where the explicit form of the operators $\mathbf{O}_{i}$ and $\mathbf{O}_{f}$ does not matter here and can be found in the article by Brambilla, Pineda, et al. (2001). 
As discussed in the previous section, the state $\psi^{\dagger}\left(\mathbf{x}_{1}\right) \phi\left(\mathbf{x}_{1}, \mathbf{x}_{2}\right) \chi_{c}^{\dagger}\left(\mathbf{x}_{2}\right) \mid$ vac $\rangle$ has a nonvanishing overlap with the NRQCD ground state $\left|\underline{0} ; \mathbf{x}_{1}, \mathbf{x}_{2}\right\rangle$ :

$$
\begin{aligned}
& Z^{1 / 2}\left(\mathbf{x}_{1}, \mathbf{x}_{2},-i \nabla_{\mathbf{x}_{1}},-i \nabla_{\mathbf{x}_{2}}\right) \delta^{(3)}\left(\mathbf{x}_{1}-\mathbf{y}_{1}\right) \delta^{(3)}\left(\mathbf{x}_{2}-\mathbf{y}_{2}\right) \\
& =\left\langle\operatorname{vac}\left|\chi_{c}\left(\mathbf{x}_{2}\right) \phi\left(\mathbf{x}_{2}, \mathbf{x}_{1}\right) \psi\left(\mathbf{x}_{1}\right)\right| \underline{0} ; \mathbf{y}_{1}, \mathbf{y}_{2}\right\rangle .
\end{aligned}
$$

Since we are only interested in the analytic terms in $1 / m$, the normalization factor $Z$ may also be expanded in $1 / m$ :

$$
\begin{aligned}
& Z\left(\mathbf{x}_{1}, \mathbf{x}_{2},-i \nabla_{\mathbf{x}_{1}},-i \nabla_{\mathbf{x}_{2}}\right) \\
&= Z^{(0)}(r)+\left(\frac{1}{m_{1}}+\frac{1}{m_{2}}\right) Z^{(1)}(r)+i Z^{(1, p)}(r) \\
& \times \mathbf{r} \cdot\left(\frac{-i \nabla_{\mathbf{x}_{1}}}{m_{1}}-\frac{-i \nabla_{\mathbf{x}_{2}}}{m_{2}}\right)+\cdots .
\end{aligned}
$$

The NRQCD ground state is the degree of freedom that we identify with the singlet field of pNRQCD. Therefore the Green's function in pNRQCD that matches $G_{\mathrm{NRQCD}}$ is

$$
\begin{aligned}
G_{\mathrm{pNRQCD}}= & \langle\operatorname{vac}| Z^{1 / 2}\left(\mathbf{x}_{1}, \mathbf{x}_{2},-i \nabla_{\mathbf{x}_{1}},-i \nabla_{\mathbf{x}_{2}}\right) \\
& \times S\left(\mathbf{x}_{1}, \mathbf{x}_{2}, T_{W} / 2\right) S^{\dagger}\left(\mathbf{y}_{1}, \mathbf{y}_{2},-T_{W} / 2\right) \\
& \times Z^{\dagger 1 / 2}\left(\mathbf{y}_{1}, \mathbf{y}_{2},-i \nabla_{\mathbf{y}_{1}},-i \nabla_{\mathbf{y}_{2}}\right)|\mathrm{vac}\rangle \\
= & Z^{1 / 2} e^{-i T_{W} h_{s}} Z^{\dagger 1 / 2} \delta^{(3)}\left(\mathbf{x}_{1}-\mathbf{y}_{1}\right) \delta^{(3)}\left(\mathbf{x}_{2}-\mathbf{y}_{2}\right) .
\end{aligned}
$$

Matching Eq. (275) with Eq. (269) we obtain at $\mathcal{O}\left(1 / m^{0}\right)$

$$
\begin{aligned}
& V^{(0)}=\lim _{T_{W} \rightarrow \infty} \frac{i}{T_{W}} \ln \left\langle W_{\square}\right\rangle, \\
& \ln Z^{(0)}=\lim _{T_{W} \rightarrow \infty}\left(\ln \left\langle W_{\square}\right\rangle+i V^{(0)} T_{W}\right) .
\end{aligned}
$$

Equation (276) coincides with Eq. (243) and is equivalent (up to US corrections) to the weak-coupling result of Sec. IV.F.2. Matching at $\mathcal{O}(1 / \mathrm{m})$ we obtain

$$
\begin{aligned}
V^{(1,0)}+\frac{1}{2}\left(\nabla_{\mathbf{r}} V^{(0)}\right) \cdot \mathbf{r} \frac{Z^{(1, p)}}{Z^{(0)}}= & \lim _{T_{W \rightarrow \infty}}\left\{-\frac{1}{8}\left(\frac{\left(\nabla_{\mathbf{r}} Z^{(0)}\right)}{Z^{(0)}}\right)^{2}+i \frac{T_{W}}{4} \frac{\left(\nabla_{\mathbf{r}} Z^{(0)}\right)}{Z^{(0)}} \cdot\left(\nabla_{\mathbf{r}} V^{(0)}\right)+\frac{T_{W}^{2}}{12}\left(\nabla_{\mathbf{r}} V^{(0)}\right)^{2}-\frac{g}{4} \int_{-T_{W} / 2}^{T_{W} / 2} d t\left[\left(1-\frac{2 t}{T_{W}}\right)\right.\right. \\
& \left.\times \frac{\left\langle\mathbf{O}_{f}\left(T_{W} / 2\right) \cdot \mathbf{E}(t)\right\rangle_{\square}}{\left\langle W_{\square}\right\rangle}-\left(1+\frac{2 t}{T_{W}}\right) \frac{\left\langle\mathbf{E}(t) \cdot \mathbf{O}_{i}\left(-T_{W} / 2\right)\right\rangle_{\square}}{\left\langle W_{\square}\right\rangle}\right]-\frac{1}{2} \frac{\left\langle\mathbf{O}_{f}\left(T_{W} / 2\right) \mathbf{O}_{i}\left(-T_{W} / 2\right)\right\rangle_{\square}}{\left\langle W_{\square}\right\rangle} \\
& \left.-\frac{g^{2}}{4 T_{W}} \int_{-T_{W} / 2}^{T_{W} / 2} d t \int_{-T_{W} / 2}^{T_{W} / 2} d t^{\prime}\left|t-t^{\prime}\right| \frac{\left\langle\mathbf{E}(t) \cdot \mathbf{E}\left(t^{\prime}\right)\right\rangle_{\square}}{\left\langle W_{\square}\right\rangle}\right\} .
\end{aligned}
$$

From Eq. (278) we cannot disentangle $V^{(1,0)}$ from $Z^{(1, p)}$. This reflects, in the framework of the Wilson-loop matching, the freedom to perform unitary field redefinitions on the pNRQCD Lagrangian. Indeed, the Green's function (275) does not uniquely define $h_{s}$, but only up to a unitary transformation of $Z$ and $h_{s}$. Note that Eq. (273) allows one to calculate $Z^{(1, p)}$ only after a procedure that fixes $\left|\underline{0} ; \mathbf{x}_{1}, \mathbf{x}_{2}\right\rangle$, which is defined up to a transformation (229), has been given. A possible choice of $Z^{(1, p)}$ is the one that fixes $V^{(1,0)}$ to the value found in Eq. (244). Here this choice appears arbitrary and no obvious criteria to prefer it seem to be at hand. Naturally, the same result would follow by calculating $Z^{(1, p)}$ from Eq. (273) with the "quantum-mechanical" procedure (228).

In the same way we perform the matching at order $1 / \mathrm{m}^{2}$. In that case, the Wilson amplitude to match would be the sum of all amplitudes made by an insertion of a $1 / m^{2}$ or of two $1 / m$ NRQCD operators. In order to fix the ambiguity between $Z$ and $h_{s}$ at order $1 / \mathrm{m}^{2}$, some prescription for the $1 / \mathrm{m}^{2}$ terms in $Z$ is required. Again we have no obvious criteria to guide us. However, with a suitable choice we would reproduce the potentials (246)-(258).
In concluding, we remark that there appear to be some advantages in using the quantum-mechanical matching rather than the direct matching of Wilson-loop amplitudes. The first one is that it provides a natural and physical procedure for calculating the potentials and the normalization factors which works for all orders in the $1 / m$ expansion. It is physical because the potentials come out independent of the initial and final interpolating fields, while dependence is encoded in the normalization factor. Moreover, the power counting is preserved. The second one is that the quantum-mechanical expressions come out manifestly finite in the large-time limit. This is not obvious for an expression similar to Eq. (278), which contains several divergent pieces that eventually cancel each other. Finally, we mention that the calculation of $V^{1 / \sqrt{m}}$ using the direct matching of Wilsonloop amplitudes has not been addressed yet.

\section{Matching of the nonanalytic terms}

In this section, we calculate $V^{1 / \sqrt{m}}$, which is the part of the potential that is nonanalytic in $1 / \mathrm{m}$. We consider real and imaginary contributions at the same time and therefore restrict ourselves to the case $m_{1}=m_{2}=m$. In 
Sec. VII.D, we have shown that $V^{1 / \sqrt{m}}$ arises from quarkantiquark pairs of relative three-momentum of order $\sqrt{m \Lambda_{\mathrm{QCD}}}$. This momentum region shows up in loops where gluons of energy $\Lambda_{\mathrm{QCD}}$ are involved (see Fig. 16). When $p \sim \Lambda_{\mathrm{QCD}}$, the scale $\sqrt{m \Lambda_{\mathrm{QCD}}}$ is the largest after $m$ and therefore the first to be integrated out from NRQCD.

Following the procedure of Brambilla, Pineda, et al. (2004), we go through these three steps.

(i) The first step is to make explicit at the level of NRQCD the existence of different degrees of freedom by splitting the quark (antiquark) field into two: a semihard field for the (three-momentum) fluctuations of $\mathcal{O}\left(\sqrt{m \Lambda_{\mathrm{OCD}}}\right), \psi_{\mathrm{sh}}\left(\chi_{\mathrm{sh}}\right)$, and a potential field for the (three-momentum) fluctuations of $\mathcal{O}(p), \psi_{p}\left(\chi_{p}\right)$ :

$$
\psi=\psi_{p}+\psi_{\mathrm{sh}}, \quad \chi=\chi_{p}+\chi_{\mathrm{sh}} .
$$

The NRQCD Lagrangian then reads

$$
L_{\mathrm{NRQCD}}=L_{\mathrm{NRQCD}}^{\mathrm{sh}}+L_{\mathrm{NRQCD}}^{p}+L_{\text {mixing }}+L_{g}+L_{l},
$$

where the Lagrangians $L_{\mathrm{NRQCD}}^{\mathrm{sh}}$ and $L_{\mathrm{NRQCD}}^{p}$ are identical to the NRQCD Lagrangian expressed in terms of semihard and potential fields, respectively, the quantities $L_{g}$ and $L_{l}$ are the NRQCD Lagrangians for gluons and light quarks, respectively, and $L_{\text {mixing }}$ contains the mixing terms.

(ii) The second step is to integrate out gluons and quarks of energy or three-momentum of $\mathcal{O}\left(\sqrt{m \Lambda_{\mathrm{QCD}}}\right)$. This leads to the EFT NRQCD':

$$
\begin{aligned}
L_{\mathrm{NRQCD}} \rightarrow L_{\mathrm{NRQCD}^{\prime}=} & L_{\mathrm{pNRQCD}}^{\mathrm{sh}}+L_{\mathrm{NRQCD}}^{p} \\
& +\operatorname{Re} L_{\text {mixing }}^{(0)}+\operatorname{Im} L_{\text {mixing }}^{(0)} \\
& +\operatorname{Re} L_{\text {mixing }}^{(1)}+\cdots+L_{g}+L_{l} .
\end{aligned}
$$

Let us discuss the different terms.

(ii.a) $L_{\mathrm{pNRQCD}}^{\mathrm{sh}}$ comes from integrating out gluons and quarks of energy or three-momentum of $\mathcal{O}\left(\sqrt{m \Lambda_{\mathrm{QCD}}}\right)$ from $L_{\mathrm{NROCD}}^{\mathrm{sh}}$. The scale $\sqrt{m \Lambda_{\mathrm{QCD}}} \gg \Lambda_{\mathrm{QCD}}$ is perturbative and therefore we can use weak-coupling techniques. If we further project onto the quark-antiquark sector, the Lagrangian $L_{\mathrm{pNRQCD}}^{\mathrm{sh}}$ will formally coincide with Eq. (60). The multipole-expanded gluons in $L_{\mathrm{pNRQCD}}^{\mathrm{sh}}$ have (four-) momentum much smaller than $\sqrt{m \Lambda_{\mathrm{QCD}}}$.

(ii.b) In order to simplify the calculation of $L_{\text {mixing, }}$, we assume

$$
\sqrt{m \Lambda_{\mathrm{QCD}}} \gg m \alpha_{s}\left(\sqrt{m \Lambda_{\mathrm{QCD}}}\right),
$$

which implies that whenever a momentum of order $\sqrt{m \Lambda_{\mathrm{QCD}}}$ flows into a Coulomb potential (note that at the scale $\sqrt{m \Lambda_{\mathrm{QCD}}}$ the potential is perturbative), the potential can be expanded about the kinetic energy. If this is not the case, then a Coulomb resummation is needed. Here we avoid the technical complications connected with this case. However, there may be situations in

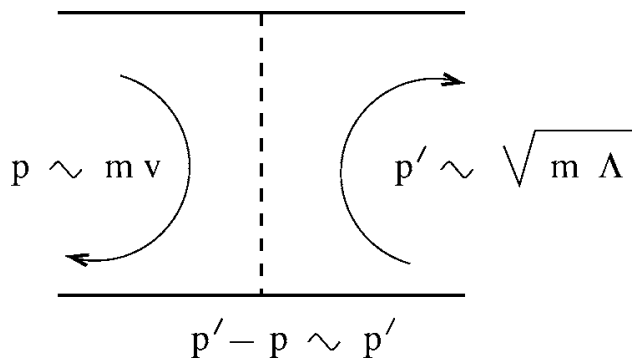

FIG. 17. The Coulomb-exchange graph contributing to the leading mixing interaction between semihard and potential fields.

which this cannot be avoided. For instance, this may be the case for the $Y$ system if the following attribution of scales holds for the $\Upsilon(1 S): p_{\Upsilon(1 S)} \sim m_{b} \alpha_{s}\left(p_{\Upsilon(1 S)}\right)$ and $\Lambda_{\mathrm{QCD}} \sim m_{b} \alpha_{s}^{2}\left(p_{\Upsilon(1 S)}\right)$, where $p_{\Upsilon(1 S)}$ is the typical momentum transfer of the $\Upsilon(1 S)$ and $m_{b}$ the bottom-quark mass. In this case one would have $\sqrt{m_{b} \Lambda_{\mathrm{QCD}}} \sim p_{\mathrm{Y}(1 S)}$ $\sim m_{b} \alpha_{s}\left(\sqrt{m_{b} \Lambda_{\mathrm{QCD}}}\right)$ instead of Eq. (282).

The leading-order contribution to the real part of $L_{\text {mixing }}$ comes from the one-Coulomb-exchange graph of Fig. 17:

$$
\begin{aligned}
& \operatorname{Re} L_{\text {mixing }}^{(0)}=-\int d^{3} \mathbf{R} \int d^{3} \mathbf{r} \operatorname{Tr}\left\{J^{\dagger}(\mathbf{R}) V_{s}^{(0)}(\mathbf{r}) S_{\mathrm{sh}}(\mathbf{R}, \mathbf{r})\right\} \\
&+ \text { H.c. }-\int d^{3} \mathbf{R} \int d^{3} \mathbf{r} \operatorname{Tr}\left\{J^{\dagger}(\mathbf{R}) V_{o}^{(0)}(\mathbf{r})\right. \\
&\left.\times O_{\text {sh }}(\mathbf{R}, \mathbf{r})\right\}+ \text { H.c. }, \\
& J^{\dagger}(\mathbf{R}) \equiv \chi_{p}(\mathbf{R}) \psi_{p}^{\dagger}(\mathbf{R}),
\end{aligned}
$$

where $S_{\mathrm{sh}}$ and $O_{\mathrm{sh}}$ are semihard singlet and octet quarkantiquark fields, respectively. The potentials $V_{s}^{(0)}$ and $V_{o}^{(0)}$ are perturbative: $V_{s}^{(0)}=-C_{F} \alpha_{s} / r$ and $V_{o}^{(0)}$ $=\left(1 / 2 N_{c}\right) \alpha_{s} / r$. The coupling constant is calculated at the semihard scale $\sqrt{m \Lambda_{\mathrm{QCD}}}$.

The leading contribution to the imaginary part of $L_{\text {mixing }}$ may be read off from the imaginary part of the pNRQCD Lagrangian at order $1 / m^{2}$ in the weakcoupling regime:

$$
\begin{aligned}
\operatorname{Im} L_{\text {mixing }}^{(0)}= & -\int d^{3} \mathbf{R} \int d^{3} \mathbf{r} \operatorname{Tr}\left\{S_{\text {sh }}^{\dagger}(\mathbf{R}, \mathbf{0}) \frac{K_{s}}{m^{2}} \delta^{(3)}(\mathbf{r}) J(\mathbf{R})\right\} \\
& + \text { H.c. }-\int d^{3} \mathbf{R} \int d^{3} \mathbf{r} \operatorname{Tr}\left\{O_{\mathrm{sh}}^{\dagger}(\mathbf{R}, \mathbf{0}) \frac{K_{o}}{m^{2}} \delta^{(3)}(\mathbf{r})\right. \\
& \times J(\mathbf{R})\}+ \text { H.c. }
\end{aligned}
$$

where

$$
\begin{aligned}
K_{s}= & -\frac{C_{A}}{2}\left\{4 \operatorname{Im} f_{1}\left({ }^{1} S_{0}\right)-2 \mathbf{S}^{2}\left[\operatorname{Im} f_{1}\left({ }^{1} S_{0}\right)-\operatorname{Im} f_{1}\left({ }^{3} S_{1}\right)\right]\right. \\
& \left.+4 \operatorname{Im} f_{\mathrm{EM}}\left({ }^{1} S_{0}\right)-2 \mathbf{S}^{2}\left[\operatorname{Im} f_{\mathrm{EM}}\left({ }^{1} S_{0}\right)-\operatorname{Im} f_{\mathrm{EM}}\left({ }^{3} S_{1}\right)\right]\right\},
\end{aligned}
$$




$$
K_{o}=-\frac{T_{F}}{2}\left\{4 \operatorname{Im} f_{8}\left({ }^{1} S_{0}\right)-2 \mathbf{S}^{2}\left[\operatorname{Im} f_{8}\left({ }^{1} S_{0}\right)-\operatorname{Im} f_{8}\left({ }^{3} S_{1}\right)\right]\right\} .
$$

The NLO term of the real part of $L_{\text {mixing }}$ in the $p / \sqrt{m \Lambda_{\mathrm{QCD}}}$ expansion is given by

$$
\begin{aligned}
\operatorname{Re} L_{\text {mixing }}^{(1)}= & -\int d^{3} \mathbf{R} \int d^{3} \mathbf{r} \operatorname{Tr}\left\{\mathbf{J}^{\dagger}(\mathbf{R}) \cdot \mathbf{r} V_{s}^{(0)}(\mathbf{r}) S_{\text {sh }}(\mathbf{R}, \mathbf{r})\right\} \\
& + \text { H.c. }-\int d^{3} \mathbf{R} \int d^{3} \mathbf{r} \operatorname{Tr}\left\{\mathbf{J}^{\dagger}(\mathbf{R}) \cdot \mathbf{r} V_{o}^{(0)}(\mathbf{r})\right. \\
& \left.\times O_{\text {sh }}(\mathbf{R}, \mathbf{r})\right\}+ \text { H.c. },
\end{aligned}
$$

$\mathbf{J}^{\dagger}(\mathbf{R}) \equiv \chi_{p}(\mathbf{R}) \frac{\stackrel{\mathbf{D}}{2}}{2} \psi_{p}^{\dagger}(\mathbf{R})$

which can be obtained by expanding the Coulomb potential of Fig. 17 in $p / p^{\prime}$. In a similar way higher-order terms may be obtained. Note that, as expected, the potential fields always appear as local currents in $L_{\text {mixing. }}$.

(iii) The final step consists of integrating out degrees of freedom of $\mathcal{O}\left(\Lambda_{\mathrm{OCD}}\right)$. This leads to the pNRQCD Lagrangian (216). How to calculate the analytic part of the potential $V^{1 / m}$ has been discussed in Secs. VII.E.1, VII.E.2, and VII.E.3. For the explicit computation of $V^{1 / \sqrt{m}}$, we refer the reader to the work of Brambilla, Pineda, et al. (2004). The results for $\operatorname{Re} V^{1 / \sqrt{m}}(r)$ and $\operatorname{Im} V^{-1 / \sqrt{m}}(r)$ turn out to be

$$
\begin{aligned}
& \operatorname{Re} V^{1 / \sqrt{m}}(r)=\left(2 C_{F}+C_{A}\right)^{2} \frac{4}{3 \Gamma(9 / 2)} \pi \alpha_{s}^{2} \mathcal{E}_{7 / 2}^{E} \frac{\delta^{(3)}(\mathbf{r})}{m^{3 / 2}}, \\
& \operatorname{Im} V^{1 / \sqrt{m}}(r)=\left(2 C_{F}+C_{A}\right) \frac{4}{3 \Gamma(7 / 2)} K_{s} \alpha_{s} \mathcal{E}_{5 / 2}^{E} \frac{\delta^{(3)}(\mathbf{r})}{m^{5 / 2}},
\end{aligned}
$$

where, in order to avoid the phase ambiguity in the definition of the fractional power of a complex number, we have written the chromoelectric correlator of Eq. (267) in Euclidean space,

$$
\mathcal{E}_{n}^{E} \equiv \frac{1}{N_{c}} \int_{0}^{\infty} d \tau \tau^{n}\langle g \mathbf{E}(t) \cdot g \mathbf{E}(0)\rangle_{E}
$$

In accordance with the power counting of Sec. VII.B, Eq. (290) gives a contribution of order $p^{3} / \mathrm{m}^{2}$ $\times m \alpha_{s} / \sqrt{m \Lambda_{\mathrm{QCD}}} \times \alpha_{s}$ and Eq. (291) gives one of order $p^{3} / m^{2} \times m \alpha_{s} / \sqrt{m \Lambda_{\mathrm{QCD}}} \times \Lambda_{\mathrm{QCD}} / m$. Therefore the correction (290) is suppressed with respect to the largest $1 / \mathrm{m}^{2}$ potentials calculated in Sec. VII.E.2. The correction (291) is suppressed with respect to the imaginary part of the $1 / \mathrm{m}^{2}$ potential, given in Eq. (75). However, its size relative to the imaginary part of the $1 / \mathrm{m}^{4}$ potential, given in Eq. (268), depends on the size of $\alpha_{s}\left(\sqrt{m \Lambda_{\mathrm{QCD}}}\right)$ about which no definite statement can be made at this point.

\section{F. Matching for $|\mathrm{p}| \gg \Lambda_{\mathrm{QCD}} \gg E$}

Although it is not clear whether quarkonia states fulfilling $|\mathbf{p}| \gg \Lambda_{\mathrm{QCD}} \gg E$ exist in nature, this situation is worth investigating. The reason is that the calculation in the $|\mathbf{p}| \gg \Lambda_{\mathrm{QCD}} \gg E$ case can be divided into two steps, the first of which can be carried out by a perturbative calculation in $\alpha_{s}$. The second step, even if it is nonperturbative in $\alpha_{s}$, admits a diagrammatic representation which makes the calculation somewhat more intuitive.

\section{1. $P N R Q C D^{\prime}$}

We shall call pNRQCD' the EFT for energies below $|\mathbf{p}|$. Since $|\mathbf{p}| \gg \Lambda_{\mathrm{QCD}}$, integrating out the energy scale $|\mathbf{p}|$, namely, the matching between NRQCD and pNRQCD', can be carried out perturbatively in $\alpha_{s}$. The resulting EFT Lagrangian entirely coincides with the pNRQCD one in the weak-coupling regime, which at lower orders has been displayed in Eqs. (60) and (74). Here we need higher-order terms in the multipole expansion (at tree level):

$$
\begin{aligned}
\delta \mathcal{L}_{\mathrm{pNRQCD}^{\prime}}= & \frac{1}{8} \operatorname{Tr}\left\{O^{\dagger} \mathbf{r}^{i} \mathbf{r}^{j} g \mathbf{D}^{i} \mathbf{E}^{j} O-O^{\dagger} O \mathbf{r}^{i} \mathbf{r}^{j} g \mathbf{D}^{i} \mathbf{E}^{j}\right\} \\
& +\frac{1}{24} \operatorname{Tr}\left\{O^{\dagger} \mathbf{r}^{i} \mathbf{r}^{j} \mathbf{r}^{k} g \mathbf{D}^{i} \mathbf{D}^{j} \mathbf{E}^{k} S+\text { H.c. }\right\} \\
& +\frac{c_{F}}{2 m} \operatorname{Tr}\left\{O^{\dagger}\left(\boldsymbol{\sigma}_{1}-\boldsymbol{\sigma}_{2}\right) \cdot g \mathbf{B} S+\text { H.c. }\right\}
\end{aligned}
$$

where the traces are in color space only. $S$ and $O$ are chosen to transform as a $1 / 2 \otimes 1 / 2$ representation in spin space (hence $\boldsymbol{\sigma}_{1}-\boldsymbol{\sigma}_{2}=\boldsymbol{\sigma}_{1} \otimes \mathbf{1}_{2}-\mathbf{1}_{1} \otimes \boldsymbol{\sigma}_{2}$ ).

\section{Matching $P N R Q C D$ to $P N R Q C D^{\prime}$}

The matching of $\mathrm{pNRQCD}^{\prime}$ to $\mathrm{pNRQCD}$ can no longer be done perturbatively in $\alpha_{s}$, but it can, indeed, be done perturbatively in the following ratios of scales: $\Lambda_{\mathrm{QCD}} /|\mathbf{p}|$ (multipole expansion), $\Lambda_{\mathrm{QCD}} / m$, and $E / \Lambda_{\mathrm{QCD}}$. Therefore the basic skeleton of the calculation consists of an expansion in $x=\left(\Lambda_{\mathrm{QCD}} /|\mathbf{p}|\right)^{2}$ and $y=\left(\Lambda_{\mathrm{QCD}} / m\right)^{2}$. This suggests writing the pNRQCD Hamiltonian as

$$
h=h_{s}+h_{x}+h_{x^{2}}+h_{y}+\cdots .
$$

The interpolating fields of $\mathrm{pNRQCD}^{\prime}$ and $\mathrm{pNRQCD}$ will be related by

$$
\begin{aligned}
\left.S\right|_{\mathrm{pNRQCD}^{\prime}} & =\left.Z^{1 / 2} S\right|_{\mathrm{pNRQCD}} \\
& =\left.\left(1+Z_{x}+Z_{x^{2}}+Z_{y}+\cdots\right)^{1 / 2} S\right|_{\mathrm{pNRQCD}} .
\end{aligned}
$$

The matching calculation is 


$$
\begin{aligned}
& \int_{-\infty}^{\infty} d t e^{-i E t} \int d^{3} \mathbf{R}\langle\operatorname{vac}| T\left\{S(\mathbf{x}, \mathbf{R}, t) S\left(\mathbf{x}^{\prime}, \mathbf{0}, 0\right)\right\} \\
& \quad \times\left.|\operatorname{vac}\rangle\right|_{\mathrm{pNRQCD}} \\
& =\int_{-\infty}^{\infty} d t e^{-i E t} \int d^{3} \mathbf{R} Z^{1 / 2}\langle\operatorname{vac}| T\left\{S(\mathbf{x}, \mathbf{R}, t) S\left(\mathbf{x}^{\prime}, \mathbf{0}, \mathbf{0}\right)\right\} \\
& \quad \times\left.|\operatorname{vac}\rangle\right|_{\mathrm{pNRQCD}} Z^{(1 / 2)^{\dagger}} .
\end{aligned}
$$

The right-hand side of the matching calculation has the following structure:

$$
\begin{gathered}
\frac{1}{E-h_{s}}+\frac{1}{E-h_{s}}\left(h_{x}+h_{x^{2}}+h_{y}\right) \frac{1}{E-h_{s}}+\frac{1}{2}\left(Z_{x}+Z_{x^{2}}\right. \\
\left.+Z_{y}-\frac{Z_{x}^{2}}{4}\right) \frac{1}{E-h_{s}}+\frac{1}{E-h_{s}} \frac{1}{2}\left(Z_{x}+Z_{x^{2}}+Z_{y}\right. \\
\left.-\frac{Z_{x}^{2}}{4}\right)^{\dagger}+\left(\frac{Z_{x}}{2}\right) \frac{1}{E-h_{s}}\left(\frac{Z_{x}}{2}\right)^{\dagger} \\
+\frac{1}{E-h_{s}} h_{x} \frac{1}{E-h_{s}} h_{x} \frac{1}{E-h_{s}} \\
+\left(\frac{Z_{x}}{2}\right) \frac{1}{E-h_{s}} h_{x} \frac{1}{E-h_{s}}+\frac{1}{E-h_{s}} h_{x} \frac{1}{E-h_{s}}\left(\frac{Z_{x}}{2}\right)^{\dagger}
\end{gathered}
$$

Hence once we have made sure that, up to contact terms, the left-hand side of Eq. (296) has exactly the same structure, we can easily identify the contributions to the pNRQCD Hamiltonian from the second term of expression (297).

Let us illustrate how the calculation of the left-hand side of Eq. (296) proceeds by concentrating on the following contribution:

$$
\frac{1}{E-h_{s}} \frac{i}{N_{c}} \int_{0}^{\infty} d t\left\langle i \mathbf{r} \cdot g \mathbf{E}(t) e^{-i\left(h_{o}-E\right) t} i \mathbf{r} \cdot g \mathbf{E}(0)\right\rangle \frac{1}{E-h_{s}} .
$$

One might naively think that because $E / \Lambda_{\mathrm{QCD}}$ is small, it can be implemented by expanding the exponential $(t$ takes the typical value of $1 / \Lambda_{\mathrm{QCD}}$ ) (Brambilla, Eiras, et al., 2002, 2003). However, this is not entirely correct. Whereas it is true that $h_{o}$, between the heavy quarkonium states we are considering, has size $E$, it may experience fluctuations of a larger size, for instance, $\sim \Lambda_{\mathrm{QCD}}$ since the cutoff of the relative three-momentum is only constrained to be smaller than $m$, and hence it may well reach values $\sim \sqrt{m \Lambda_{\mathrm{QCD}}}$. Nevertheless, the energy $E$ can indeed always be expanded, which guarantees that we eventually get usual, energy-independent, potentials. If $h_{o}$ could not be expanded, we obtain potentials which are nontrivial functions of $m, \Lambda_{\mathrm{QCD}}$, and $\mathbf{r}$. Fortunately, we can do much better by exploiting the fact that the momenta, which prevent us from expanding, fulfill $|\mathbf{p}|$ $\sim \sqrt{m \Lambda_{\mathrm{QCD}}} \gg \Lambda_{\mathrm{QCD}}$. We shall proceed as follows. We split the relative momentum into two regions. The first region fulfills $|\mathbf{p}| \ll \sqrt{m \Lambda_{\mathrm{QCD}}}$ and $h_{o}$ can be expanded and the second region contains the momentum fluctuations $\sim \sqrt{m \Lambda_{\mathrm{QCD}}}$.

(i) The matching in the region $|\mathbf{p}| \ll \sqrt{m \Lambda_{\mathrm{QCD}}}$.

(i.a) The real part of the potential.

At LO in the expansion, the exponential in Eq. (298) reduces to 1 and we obtain the leading nonperturbative correction to the Coulomb potential:

$$
\delta V_{s}=-i \frac{g^{2}}{N_{c}} T_{F} \frac{r^{2}}{3} \int_{0}^{\infty} d t\left\langle\mathbf{E}^{a}(t) \phi(t, 0)_{a b}^{\mathrm{adj}} \mathbf{E}^{b}(0)\right\rangle .
$$

This expression was first derived by Balitsky (1985). Higher orders in the $E / \Lambda_{\mathrm{QCD}}$ expansion can be easily calculated. They induce contributions to potentials which are higher order in $1 / \mathrm{m}$ as well as further contributions to the static potential. Some of these have been calculated by Brambilla et al. (2000).

(i.b) The imaginary part of the potential.

Since the imaginary parts, which are inherited from NRQCD, are contained in local $\left[\delta^{(3)}(\mathbf{r}), \boldsymbol{\nabla} \delta^{(3)}(\mathbf{r}) \nabla\right.$, etc. $]$ terms in the $\mathrm{pNRQCD}$ ' Lagrangian, they tend to vanish when being multiplied by the r's arising from the multipole expansion. Hence for an imaginary part to contribute, it must have a sufficient number of derivatives (usually arising from the $E / \Lambda_{\mathrm{QCD}}$ expansion) in order to cancel all the r's. Since derivatives are always accompanied by powers of $1 / m$, it implies that at a given order in $1 / m$, only a finite number of terms in the multipole expansion contributes. We are only interested in collecting the imaginary parts that contribute up to order $1 / \mathrm{m}^{4}$ in order to provide an independent calculation to support the results of Sec. VII.E. Consider again the contribution of Eq. (298). The first imaginary terms arise at $\mathcal{O}\left(E / \Lambda_{\mathrm{QCD}}\right)$ from the $\mathcal{O}\left(1 / \mathrm{m}^{4}\right)$ parts of the singlet and octet potentials displayed in Eq. (76):

$$
\begin{aligned}
& \frac{i}{E-} h_{s}\left(\frac{T_{F} \mathcal{T}_{S J}^{i i} \operatorname{Im} f_{8}\left({ }^{2 S+1} P_{J}\right)}{3 N_{c} m^{4}}\right. \\
& \left.+\frac{\mathcal{T}_{S}\left[\operatorname{Im} g_{1}\left({ }^{2 S+1} S_{S}\right)+\operatorname{Im} g_{\mathrm{EM}}\left({ }^{2 S+1} S_{S}\right)\right]}{m^{4}}\right) \\
& \quad \times \int_{0}^{\infty} d t t\langle g \mathbf{E}(t) \cdot g \mathbf{E}(0)\rangle \frac{\delta^{(3)}(\mathbf{r})}{E-h_{s}},
\end{aligned}
$$

where $\mathcal{T}_{S J}^{i j}$ are defined are defined in Eqs. (77)-(80) and $\mathcal{T}_{S}=\Omega_{S S}^{i i} / 3$. The calculation may be systematically extended to higher orders. Details are given by Brambilla, Eiras, et al. (2003). Here we just point out two subtleties. First, ill-defined expressions arise in the calculation from products of distributions (both products of two delta functions and products of delta functions with nonlocal potentials, which diverge as $\mathbf{r} \rightarrow 0$ ). It is most convenient to use DR in this case, which sets all these terms to zero. This is shown in Appendix D in the article by Brambilla, Eiras, et al. (2003), where the relation to other regularization schemes is also discussed. Second, there is a freedom in organizing the calculation, which may lead to different forms of the potentials. Let us consider, as an example, the term 


$$
\frac{1}{E-h_{s}} \mathbf{r}\left(E-h_{s}\right)^{2} \mathbf{r} \frac{1}{E-h_{s}} .
$$

If we decide to take one power $\left(E-h_{s}\right)$ to the right and one to the left, we have

$$
\begin{aligned}
\mathbf{r}^{2}+ & \mathbf{r}\left[\mathbf{r}, h_{s}\right] \frac{1}{E-h_{s}}+\frac{1}{E-h_{s}}\left[h_{s}, \mathbf{r}\right] \mathbf{r}+\frac{1}{E-h_{s}}\left[h_{s}, \mathbf{r}\right] \\
& \times\left[\mathbf{r}, h_{s}\right] \frac{1}{E-h_{s}},
\end{aligned}
$$

which does not produce any imaginary part. However, an equally acceptable expression is

$$
\begin{aligned}
\mathbf{r}^{2}+ & \frac{1}{2}\left[\mathbf{r},\left[\mathbf{r}, h_{s}\right]\right] \frac{1}{E-h_{s}}+\frac{1}{E-h_{s}} \frac{1}{2}\left[\left[h_{s}, \mathbf{r}\right], \mathbf{r}\right] \\
& +\frac{1}{E-h_{s}} \frac{1}{2}\left\{\left[\left[\mathbf{r}, h_{s}\right], h_{s}\right], \mathbf{r}\right\} \frac{1}{E-h_{s}},
\end{aligned}
$$

which does produce an imaginary part. The apparent paradox only reflects the fact that expression (301) by itself (as well as others from the calculation) does not determine uniquely its contribution to the potential. It leads to contact terms, wave-function normalization and potential, as is apparent in Eqs. (302) and (303), but depending on how we decide to organize the calculation, the terms associated with each of these pieces change. For instance, when matched to Eq. (297), Eq. (302) gives $h_{x}=\left[h_{s}, \mathbf{r}\right]\left[\mathbf{r}, h_{s}\right], Z_{x}=\mathbf{r}\left[\mathbf{r}, h_{s}\right]$, whereas Eq. (303) gives $h_{x}$ $=\frac{1}{2}\left\{\left[\left[\mathbf{r}, h_{s}\right], h_{s}\right], \mathbf{r}\right\}, Z_{x}=\frac{1}{2}\left[\mathbf{r},\left[\mathbf{r}, h_{s}\right]\right]$. This should not be a surprise. It corresponds to the freedom of making unitary transformations in a quantum-mechanical Hamiltonian already discussed in the previous sections, and does not affect any physical observables. In order to fix the contribution to the potential of any term once and forever, we use the prescription described in detail in Sec. V of Brambilla, Eiras, et al. (2003). With this prescription, Eq. (301) gives rise to the potential obtained in Eq. (302) and hence to no imaginary part. Eventually, combining all the contributions, we obtain for the imaginary part of the pNRQCD potential when $p \gg \Lambda_{\mathrm{QCD}}$ $\gg E$ the same result, up to a unitary transformation, as obtained in Sec. VII.E when $p \sim \Lambda_{\mathrm{QCD}}$ and explicitly listed in Eqs. (75) and (268). The explicit form of the unitary transformation can be found in the article by Brambilla, Eiras, et al. (2003).

(ii) The matching in the region $|\mathbf{p}| \sim \sqrt{m \Lambda_{\mathrm{QCD}}}$.

The contributions due to heavy quarks of threemomentum of order $\sqrt{m \Lambda_{\mathrm{QCD}}}$ may be calculated similar to Sec. VII.E.5. The main difference is that now potential and semihard degrees of freedom need not be separated at the level of NRQCD, but of pNRQCD'.

(ii.a) The first step consists in rewriting the $\mathrm{pNRQCD}^{\prime}$ Lagrangian in terms of semihard fields $S_{\mathrm{sh}}$ and $O_{\mathrm{sh}}^{a}$ associated with three-momentum fluctuations of $\mathcal{O}\left(\sqrt{m \Lambda_{\mathrm{QCD}}}\right)$ and potential fields $S_{p}$ and $O_{p}^{a}$ associated with three-momentum fluctuations of $\mathcal{O}(p)$ :

$$
S=S_{p}+S_{\mathrm{sh}}, \quad O^{a}=O_{p}^{a}+O_{\mathrm{sh}}^{a} .
$$

The pNRQCD' Lagrangian is then

$$
\begin{aligned}
L_{\mathrm{pNRQCD}^{\prime}}= & L_{\mathrm{pNRQCD}^{\prime}}^{\mathrm{sh}}+L_{\mathrm{pNRQCD}^{\prime}}^{p}+L_{\text {mixing }}+L_{g} \\
& +L_{l},
\end{aligned}
$$

where $L_{\mathrm{pNRQCD}}^{\mathrm{sh}}$ and $L_{\mathrm{pNRQCD}}^{p}$ are identical to the pNRQCD $^{\prime}$ Lagrangian in the heavy-quarkonium bilinear sector except for the changes $S, O^{a}, V_{s}, V_{o} \rightarrow S_{\mathrm{sh}}$, $O_{\mathrm{sh}}^{a}, V_{s}^{\mathrm{sh}, \mathrm{sh}}, V_{o}^{\mathrm{sh}, \mathrm{sh}}$, and $S, O^{a}, V_{s}, V_{o} \rightarrow S_{p}, O_{p}^{a}, V_{s}^{p, p}, V_{o}^{p, p}$, respectively. $L_{g}$ and $L_{l}$ are the parts of the pNRQCD' Lagrangian that contain only gluons and light quarks, respectively, and $L_{\text {mixing }}$ contains the mixing terms. We recall that the gluons left dynamical have energies of $\mathcal{O}\left(\Lambda_{\mathrm{QCD}}\right)$ and that analytic terms in $\mathbf{r}$ do not mix semihard and potential fields. Therefore the multipole expansion in Eq. (293) is an expansion in either the scale $\mathbf{r} \sim 1 / \sqrt{m \Lambda_{\mathrm{QCD}}}$ in $L_{\mathrm{pNRQCD}}^{(\mathrm{sh})}$ or the scale $\mathbf{r} \sim 1 / p$ in $L_{\mathrm{pNRQCD}}^{p}$

(ii.b) The second step consists of integrating out gluons and quarks of energy and three-momentum of $\mathcal{O}\left(\sqrt{m \Lambda_{\mathrm{QCD}}}\right)$. We assume, as in Eq. (282) and for the same reasons as discussed there, that $\sqrt{m \Lambda_{\mathrm{QCD}}}$ $\gg m \alpha_{s}\left(\sqrt{m \Lambda_{\mathrm{QCD}}}\right)$. As an example, we consider the real part of the singlet mixing term due to the static Coulomb potential. The matching works exactly as in paragraph (ii.b) of Sec. VII.E.5 and leads to

$$
\begin{aligned}
\text { Re } & \left.L_{\text {mixing }}\right|_{\text {singlet }} \\
= & -\int d^{3} \mathbf{R} \int d^{3} \mathbf{r} S_{p}^{\dagger}(\mathbf{R}, \mathbf{r}) V_{s}^{(0)}(\mathbf{r}) S_{\mathrm{sh}}(\mathbf{R}, \mathbf{r})+\text { H.c. } \\
\rightarrow & -\int d^{3} \mathbf{R} \int d^{3} \mathbf{r}\left[S_{p}^{\dagger}(\mathbf{R}, \mathbf{0})+\mathbf{r} \cdot \nabla_{\mathbf{r}} S_{p}^{\dagger}(\mathbf{R}, \mathbf{0})+\cdots\right] \\
& \times V_{s}^{(0)}(\mathbf{r}) S_{\mathrm{sh}}(\mathbf{R}, \mathbf{r})+\text { H.c. }
\end{aligned}
$$

At the order of interest, we have $V_{s}^{(0)}=-C_{F} \alpha_{s} / r$ and $\alpha_{s}$ $=\alpha_{s}\left(\sqrt{m \Lambda_{\mathrm{QCD}}}\right)$. Analogous results hold for the real part of the octetmixing term due to the static Coulomb potential.

The leading contribution to the imaginary part of $L_{\text {mixing }}$ is given by

$$
\begin{aligned}
\operatorname{Im} L_{\text {mixing }}= & -\int d^{3} \mathbf{R} \int d^{3} \mathbf{r} \operatorname{Tr}\left\{S_{\mathrm{sh}}^{\dagger}(\mathbf{R}, \mathbf{0}) \frac{K_{s}}{m^{2}} \delta^{(3)}(\mathbf{r})\right. \\
& \left.\times S_{p}(\mathbf{R}, \mathbf{0})+\text { H.c. }\right\} \\
& -\int d^{3} \mathbf{R} \int d^{3} \mathbf{r} \operatorname{Tr}\left\{O_{\mathrm{sh}}^{\dagger}(\mathbf{R}, \mathbf{0}) \frac{K_{o}}{m^{2}} \delta^{(3)}(\mathbf{r})\right. \\
& \left.\times O_{p}(\mathbf{R}, \mathbf{0})+\text { H.c. }\right\},
\end{aligned}
$$

where $K_{s}$ and $K_{o}$ have been defined in Eqs. (286) and (287), respectively.

(ii.c) The final step consists in integrating out from pNRQCD' all fluctuations that appear at the energy 
scale $\Lambda_{\mathrm{QCD}}$. These are light quarks and gluons of energy or three-momentum of order $\Lambda_{\mathrm{QCD}}$, and singlet and octet fields of energy of order $\Lambda_{\mathrm{OCD}}$ or three-momentum of order $\sqrt{m \Lambda_{\mathrm{QCD}}}$. We are then left with pNRQCD. The part $V^{1 / m}$ of the potential [see Eq. (220)] has been calculated in paragraph (i) of this section. The part $V^{1 / \sqrt{m}}$ of the potential develops a real and an imaginary part. They turn out to be equal to Eqs. (290) and (291), respectively, i.e., to the results obtained in the kinematical situation $p \sim \Lambda_{\mathrm{QCD}}$. We refer the reader to Brambilla, Pineda, et al. (2004) for a detailed diagrammatical calculation.

In summary, we have presented a derivation of the pNRQCD potential (real and imaginary) in a kinematical situation and with a technical procedure that are quite different from the ones of Sec. VII.E. The agreement of the results (up to unitary transformations) in the case when the potentials are local (nonanalytic and imaginary terms) is reassuring and confirms in an explicit calculation what is expected in Sec. VII.D on general grounds. Despite this, it should be noted that the matching coefficients of the terms in the multipole expansion in PNRQCD' $^{\prime}$ (293) were only calculated at tree level here, whereas the expressions in Sec. VII.E correspond to an all-order result. This indicates that there must be a symmetry protecting these terms against higher-loop corrections. ${ }^{24}$ This symmetry does not appear to be Poincaré invariance (Brambilla, Gromes, and Vairo, 2003).

\section{G. Potentials and spectra: lattice and models}

The heavy-quarkonium spectrum is obtained by solving the Schrödinger equation for the pNRQCD Hamiltonian $h_{s}$ :

$$
h_{s} \phi_{n j l s}(\mathbf{r})=E_{n j l s} \phi_{n j l s}(\mathbf{r}) .
$$

Since $h_{s}$ is known from Eqs. (243)-(258), (75), (268), (290), and (291), the Schrödinger equation (308) is completely defined in terms of QCD quantities.

At LO, Eq. (308) becomes

$$
\begin{aligned}
h_{s}^{(0)} \phi_{n j l s}^{(0)}(\mathbf{r}) & =\left(\frac{\mathbf{p}_{1}^{2}}{2 m_{1}}+\frac{\mathbf{p}_{2}^{2}}{2 m_{2}}+V_{\mathrm{LO}}\right) \phi_{n j l s}^{(0)}(\mathbf{r}) \\
& =E_{n j l s}^{(0)} \phi_{n j l s}^{(0)}(\mathbf{r}) .
\end{aligned}
$$

What $V_{\mathrm{LO}}$ is depends on the power counting. We have argued in Sec. VII.E.2 that when $p \sim \Lambda_{\mathrm{QCD}}$ and in the most conservative power counting, we have $V_{\mathrm{LO}}=V^{(0)}$ $+V^{(1)} / m$. On the other hand, if $p \gg \Lambda_{\mathrm{QCD}}$, we have $V_{\mathrm{LO}}$ $=V^{(0)}$. In both cases, at this order the potential is spin independent $\left(E_{n j l s}^{(0)} \equiv E_{n l}^{(0)}\right)$ and therefore the leadingorder $S$ - and $P$-wave functions read

\footnotetext{
${ }^{24}$ For the leading-order term, the nonrenormalization was verified at one loop by Pineda and Soto (2001).
}

$$
\begin{aligned}
& \phi_{n s 0 s}^{(0)}(\mathbf{r})=R_{n 0}^{(0)}(r) \frac{1}{\sqrt{4 \pi}}|s\rangle_{\text {spin }} \text { and } \\
& \phi_{n j 1 s}^{(0)}(\mathbf{r})=R_{n 1}^{(0)}(r)\langle\hat{\mathbf{r}} \mid j s\rangle,
\end{aligned}
$$

where $|s\rangle_{\text {spin }}$ denotes the normalized spin component, $|\hat{\mathbf{r}}\rangle$ the normalized eigenstate of the position, and $|j s\rangle$ the $J$ (total angular momentum) and $S$ eigenstate such that $\langle\hat{\mathbf{r}} \mid j 0\rangle=Y_{j}^{m}(\hat{\mathbf{r}})|0\rangle_{\text {spin }}(j=l=1)$ and $\langle\hat{\mathbf{r}} \mid j 1\rangle=\mathcal{Y}_{j m}^{1}(\hat{\mathbf{r}})$. The label $m$ denotes the third component of the angular momentum.

At NLO, the $1 / \mathrm{m}^{2}$ potentials calculated in Sec. VII.E.2 have to be considered, except for the ones that may have extra suppression. The contribution to the spectrum that comes from the $V^{1 / \sqrt{m}}$ potential given in Eq. (289) also turns out to be suppressed. Indeed, we have ( $m^{\text {red }}$ is the reduced mass)

$\delta E_{n j l s}^{1 / \sqrt{m}}=\left(2 C_{F}+C_{A}\right)^{2} \frac{1}{3 \Gamma(9 / 2)} \alpha_{s}^{2} \mathcal{E}_{7 / 2}^{E} \frac{\left|R_{n l}(0)\right|^{2}}{\left(2 m^{\mathrm{red}}\right)^{3 / 2}} \delta_{l 0}$,

which is of order $|\mathbf{p}|^{3} / m^{2} \times m \alpha_{s} / \sqrt{m \Lambda_{\mathrm{QCD}}} \times \alpha_{s}$, i.e., suppressed with respect to the contribution coming from the $1 / m^{2}$ potentials of Eqs. (243)-(258), which in the conservative counting is of order $\mathrm{p}^{3} / \mathrm{m}^{2}$.

We would like to emphasize that in order to be consistent with the power counting, subleading terms in the expansion of the kinetic energy and the potential should be treated as perturbations when solving Eq. (309). This differs from the common practice in potential models. In an EFT framework, the calculation of the spectrum is not plagued by the inconsistencies emerging in higherorder calculations in potential models. It is, for instance, known that at second order in quantum-mechanical perturbation theory the spin-dependent terms result in a contribution that is ill defined. Regulating it requires the introduction of a cutoff (or DR). A large cutoff gives rise to a linear and to a logarithmic divergence. These divergences can be renormalized by redefining the coupling constant of a delta potential (Lepage, 1997). On the other hand, when one matches QCD to NRQCD, one expands in the energy and the three-momentum. In general, this induces IR divergences in the matching coefficients and, in particular, in the calculation of a matching coefficient of a four-fermion operator at two loops, which leads to the delta potential mentioned above. If one uses a consistent regularization scheme for both the QCD-NRQCD matching calculation and the quantummechanical calculation in $\mathrm{pNRQCD}$, the divergences exactly cancel and eventually a totally consistent scaleindependent result is obtained [for a QED example, see Czarnecki et al. (1999a, 1999b)]. Notice that an EFT framework is crucial for understanding this secondorder calculation and for making the result meaningful.

For a determination of the spectrum at order $p^{3} / \mathrm{m}^{2}$ in the conservative counting, one needs to consider, besides the static and the $1 / m$ potential, the $\mathcal{O}\left(1 / \mathrm{m}^{2}\right)$ potentials given in Eq. (63), of which for $V_{r}^{(2,0)}$ and $V_{r}^{(1,1)}$ only the terms in the first three lines of Eqs. (248) and (252) need to be considered. How can one get the ex- 
plicit form of these potentials? The EFT provides the expressions for such potentials in terms of Wilson-loop amplitudes typically involving chromoelectric- and chromomagnetic-field insertions. In the case of the imaginary parts, they reduce to chromoelectric and chromomagnetic correlators. These are low-energy objects that do not depend on the quarkonium state, involve only integrations over gluon fields and light quarks, are gauge invariant, and perfectly suited for lattice calculations. We emphasize that the EFT approach greatly reduces the lattice effort necessary to produce heavy-quarkonium spectra and decay widths. This is for two reasons. The first reason is that the objects to be calculated on the lattice involve only integrations over low-energy gluons and light quarks. The second is that one does not need to repeat a lattice evaluation for each quarkonium state (with the problems related to the mass extraction of the excited states) but only to extract the form of all the potentials with one simulation. These, once inserted in the Schrödinger equation (309), will produce the spectrum. One should check a posteriori which states in the obtained spectrum fulfill the hypothesis of the strong-coupling regime. The ones that do will be the ones for which the calculation is reliable.

\section{Potentials and spectrum from the lattice}

If DR is used in the continuum, the Wilson-loop amplitudes involved in the static and $1 / \mathrm{m}$ potentials can be renormalized by the counterterms of light degrees of freedom only, and hence they do not display a factorization scale dependence. For the $1 / \mathrm{m}^{2}$ and higher potentials, counterterms involving local potentials are also necessary and the Wilson-loop amplitudes depend on the factorization scale. In a physical observable, this scale dependence, together with the one induced by the quantum-mechanical perturbation theory, will cancel against the scale dependence of the NRQCD matching coefficients. In the strong-coupling regime, there are no US divergences, at least when the US degrees of freedom (pseudo-Goldstone bosons) are neglected.

In a lattice regularization scheme, the situation is more complicated for several reasons. The Wilson-loop amplitudes contain additive 1/ $a$-dependent self-energy contributions ( $a$ being the lattice spacing), even in the static case. This dependence on $1 / a$ is canceled by the quark-mass shift and is removed by a suitable renormalization condition (see the discussion on the static potential in Sec. VI and below). Moreover, large terms are generated having their origin in self-interactions within the plaquette as well as between plaquette and static propagator (to higher orders). These affect all Wilsonloop amplitudes. They would be canceled by NRQCD matching coefficients calculated in a lattice regularization. Without those the scale dependence can be dramatic and several ad hoc lattice methods have been applied to get rid of it without actually calculating the matching coefficients, which would be the definite solution. In addition, the Wilson-loop amplitudes will generate $a$ - and $r$-dependent terms which are specific to the lattice. On top of this, Lorentz invariance is broken on the lattice. Thus order $a$ corrections to coefficients otherwise protected by Lorentz invariance may appear.

All these issues are related to the lattice regularization and renormalization. A proper treatment would require the calculation of both the NRQCD matching coefficients and the Wilson-loop amplitudes in a proper lattice regularization and renormalization scheme. The Schrödinger equation would also need to be solved in the same scheme, due to the quantum-mechanical divergences. The NRQCD matching coefficients are known at different accuracy in the continuum and in DR, see Sec. II.D, but up to now no calculation of the coefficients here relevant exists within a lattice scheme apart from the one in the work of Trottier and Lepage (1998). Another strategy would be to use a nonperturbative renormalization (Martinelli et al., 1997) on both parts in lattice regularization. Alternatively, if the available MS NRQCD matching coefficients are to be used, one should change the Wilson-loop amplitudes from the lattice renormalization scheme to MS. This can be done in lattice perturbation theory since the cutoff of these divergences is close to $m$ (Bodwin et al., 2002). Then the divergences arising in the quantum-mechanical perturbation theory should also be MS renormalized.

A proper lattice treatment of pNRQCD has so far not been implemented. NRQCD matching coefficients were never considered in the lattice calculation of the potentials with the exception of the work of Bali et al. (1997) and Bali (2001), in which an estimate of the NRQCD matching coefficients was used. Therefore this work may be considered the closest to a lattice treatment of pNRQCD. We shall mainly refer to it in the following.

The static potential is given only in terms of the static Wilson loop (243) and it has been one of the first objects to be evaluated on the lattice in relation to quark confinement (Wilson, 1974). Today the static potential is known with great accuracy (Bali et al., 1997; Bali, 2001; Lüscher and Weisz, 2002; Necco and Sommer, 2002) even in the unquenched case (Bali et al., 2000; Bolder et al., 2001). In Fig. 4, the curve labeled $\Sigma_{g}^{+}$displays the static-potential data obtained by Bali et al. (2000) in units of $r_{0} \approx 0.5 \mathrm{fm}$. The squares refer to a quenched simulation at $\beta=6.2$ and the bullets to unquenched simulations at $\beta=5.6$ with two mass-degenerate quark flavors. The value of the mass parameter is $\kappa=0.1575$. The physical units follow from a choice of the lattice spacing $a$. This is often fixed on the bottomonium spectrum (Bali et al., 1997; Bali, 2001). This procedure may potentially introduce large uncertainties if the set of potentials at our disposal is not complete, if the power counting not consistent, or if as is usually done, lower and higher bottomonium states are fitted with the same confining potentials. However, such a determination seems to be numerically in agreement with others obtained from the $m_{\pi} / m_{\rho}$ ratio. The continuous curve in Fig. 4 represents the Cornell parametrization $V^{(0)}(r)=-e / r+\sigma r$ with $e$ $\approx 0.368$ and $\sigma \approx(445 \mathrm{MeV})^{2}$. An additive self-energy contribution, associated with the static sources and di- 
verging in the continuum limit, has been removed by normalizing the data to $V\left(r_{0}\right)=0$. This corresponds to the elimination of the static-potential renormalon described in Sec. V. As shown in Sec. VI, QCD perturbation theory perfectly agrees with the lattice data up to about $0.25 \mathrm{fm}$ [actually the analysis done by Pineda (2003b) shows agreement up to $0.4 \mathrm{fm}$ ], while from about $0.5 \mathrm{fm}$ on the data are described very well by an effective string theory at NLO (Lüscher and Weisz, 2002). However, this seems to be specific to the ground-state energy: the energy spectrum is still far from being stringlike at such distances (Lüscher and Weisz, 2004). This is more apparent for the excited-state energies (Baker and Steinke, 2001).

For the potential at order 1/ $\mathrm{m}$ given in Eqs. (244) and (245), no lattice evaluation is available yet. The spindependent $1 / \mathrm{m}^{2}$ potentials instead have a quite long record of calculations (Campostrini, 1985; Michael, 1986; Huntley and Michael, 1987; Born et al., 1994; Bali et al., 1997). In the absence of a proper implementation of the NRQCD matching coefficients, the method proposed by Huntley and Michael (1987) was to obtain lattice spacing and scale-independent results for the spin-dependent Wilson-loop potentials based on the substitutions $\langle\langle F F\rangle\rangle \rightarrow\langle\langle F F\rangle\rangle /\langle F F\rangle, F$ being the gluon field strength. The notations used by Bali (2001) for the spindependent and momentum-dependent Wilson-loop potentials differ from what we presented in Sec. VII.E.2. The objects that were evaluated on the lattice were $V_{1}^{\prime}(r)$ [equal to $-r$ times the first term on the right-hand side of Eq. (253) with $c_{F}=1$ ] and $V_{2}^{\prime}(r)$ [equal to $-r$ times the right-hand side of Eq. (255) with $\left.c_{F}=1\right]$ for the spinorbit, $V_{3}(r)$ [equal to the first term on the right-hand side of Eq. (257) with $\left.c_{F}=1\right]$ for the spin-spin, and $V_{4}(r)$ [equal to the right-hand side of Eq. (258) with $c_{F}=1$ ] for the tensor potential. All the lattice determinations of the spin-dependent potentials use the correct expression for the spin-orbit potential (see comments in Sec. VII.E.2). An example is shown in Fig. 18(a). For the momentumdependent part, the objects evaluated on the lattice were $V_{b}=-2 / 3 V_{\mathbf{L}^{2}}^{(1,1)}-V_{\mathbf{p}^{2}}^{(1,1)}, V_{c}=-V_{\mathbf{L}^{2}}^{(1,1)}, V_{d}=V_{\mathbf{p}^{2}}^{(2,0)}+2 / 3 V_{\mathbf{L}^{2}}^{(2,0)}$, and $V_{e}=V_{\mathbf{L}^{2}}^{(2,0)}$. An example is shown in Fig. 18(b). The spin-independent and momentum-independent potentials at order $1 / \mathrm{m}^{2}$ have not yet been calculated.

The Poincaré invariance constraints (259)-(261) (which in the above notation are $V_{2}^{\prime}-V_{1}^{\prime}=V_{0}^{\prime}, V_{b}+2 V_{d}$ $=r V^{(0) \prime} / 6-V^{(0)} / 2$, and $\left.V_{c}+2 V_{e}=-r V^{(0)} / / 2\right)$ have been used to test the quality and the continuum limit of the lattice simulation by Bali et al. (1997). The lattice data satisfy well the relations especially in the short and medium range. For the long range, the data become noisy. We refer the reader to the original literature for more details.

More lattice plots may be found in the articles of Bali et al. (1997) and Bali (2001). In general the lattice curves appear to be quite noisy for large interquark separations. This calls for new determinations in a fully consistent lattice renormalization context. The lattice data have been compared with fits motivated in the short
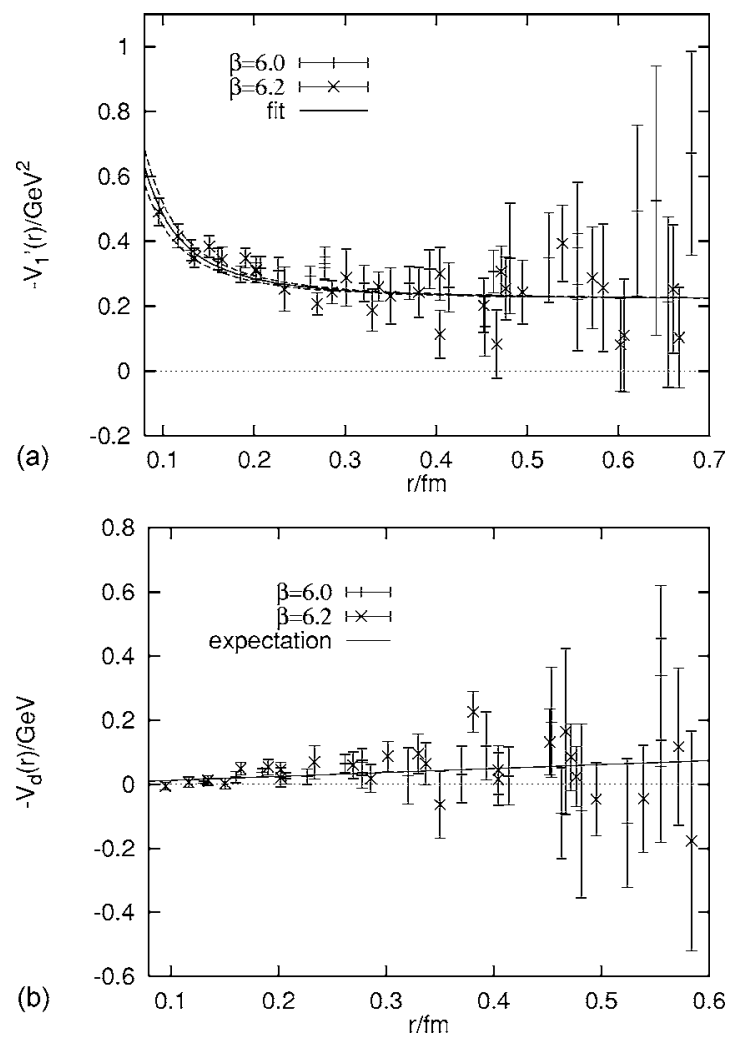

FIG. 18. (a) The spin-orbit potential $-V_{1}^{\prime}$ with the fit $\sigma+h / r^{2}$ and (b) the potential $V_{d}$ together with the curve $-\sigma / 9 r$. The lattice simulations are quenched. The fitting parameters are $\sigma \approx(468 \mathrm{MeV})^{2}$ and $h \approx 0.067$. From Bali et al., 1997 .

range by the perturbative behavior and in the long range by QCD vacuum-model calculations. We briefly mention some of them in the next subsection.

\section{QCD vacuum models}

The EFT has allowed us to systematically encode the low-energy contributions to the potentials into Wilsonloop amplitudes. These are also very convenient objects for evaluation in a QCD vacuum model. A QCD vacuum model may be defined by the behavior that it attributes to (not necessarily static) Wilson-loop expectation values in the large-distance region. Once this is known, it is possible to obtain all Wilson-loop amplitudes with field-strength insertions by means of functional derivatives of the Wilson loop (Migdal, 1983; Brambilla et al., 1994). In this way the nonperturbative form of all potentials is derived from only one assumption on the Wilson-loop behavior. The lattice data on the potentials can be compared with the expectations from different QCD vacuum models. We note that more knowledge may be gained here on the mechanism of confinement. Indeed, while all models predict confinement and thus a linear increase of the static potential, the predictions for the relativistic corrections to the static potential vary and give nontrivial information. We refer the reader to Brambilla and Vairo (1997) for calculations within the stochastic vacuum model (Dosch and Simonov, 1988), to Baker, Ball, Brambilla, Prosperi, et al. 
(1996) and Baker, Ball, Brambilla, and Vairo (1996) for calculations inside dual QCD (dual superconductor mechanism of confinement; Baker et al., 1991), to Baker et al. (1998) for a comparison between the two, and to Brambilla (1998) for a comparison also with the fluxtube model (Isgur and Paton, 1985) and the BetheSalpeter NR reduction of a scalar confining kernel. In the articles of Brambilla and Vairo (1999a, 2000b), one will find reviews of several QCD vacuum models and results relevant to the nonperturbative behavior of the potentials.

\section{H. Inclusive decay widths into light particles}

The inclusive decay width of a heavy quarkonium $H$ into light particles is (at LO in $\operatorname{Im} h_{s}$ )

$$
\Gamma(H \rightarrow \text { light particles })=-2\left\langle n, l, s, j\left|\operatorname{Im} h_{s}\right| n, l, s, j\right\rangle .
$$

The imaginary part of the pNRQCD Hamiltonian has been written in Eqs. (75), (268), and (291), and the wave functions $\phi_{n j l s}(\mathbf{r})=\langle\mathbf{r} \mid n, l, s, j\rangle$ have been discussed in Sec. VII.G. For present purposes, a LO calculation is sufficient for $P$-wave functions, while a NLO analysis, which involves the $1 / \mathrm{m}^{2}$ potentials, is necessary for $S$-wave ones.

With the above specifications and from Eq. (312), we can now list the pNRQCD expressions for $S$ - and $P$-wave decays. We proceed as follows. First, we give the expressions for the matrix elements of NRQCD that appear in Eqs. (42)-(47) distinguishing between terms that are analytic in $1 / \mathrm{m}$ and terms that are not $(\langle H|O| H\rangle$ $\left.=\langle H|O| H\rangle^{1 / m}+\langle H|O| H\rangle^{1 / \sqrt{m}}\right)$, since as we have seen in the previous sections they have been calculated in pNRQCD to different precision. Finally, we explicitly give the decay widths in $\mathrm{pNRQCD}$ at the precision to which they are presently known.

The analytic contributions in $1 / m$ to the NRQCD matrix elements have been calculated up to (once normalized to $\left.m^{0}\right) \mathcal{O}\left(p^{3} / m^{3} \times\left(\Lambda_{\mathrm{OCD}}^{2} / m^{2}, E / m\right)\right)$ for $S$-wave (Brambilla, Eiras, et al., 2003) and up to $\mathcal{O}\left(p^{5} / m^{5}\right)$ for $P$-wave matrix elements (Brambilla, Eiras, et al., 2002):

$$
\begin{aligned}
& \left\langle V_{Q}(n S)\left|O_{1}\left({ }^{3} S_{1}\right)\right| V_{Q}(n S)\right\rangle^{1 / m} \\
& =C_{A} \frac{\left|R_{n 0}^{V}(0)\right|^{2}}{2 \pi}\left(1-\frac{E_{n 0}^{(0)}}{m} \frac{2 \mathcal{E}_{3}}{9}+\frac{2 \mathcal{E}_{3}^{(2, t)}}{3 m^{2}}+\frac{c_{F}^{2} \mathcal{B}_{1}}{3 m^{2}}\right) \\
& \left\langle P_{Q}(n S)\left|O_{1}\left({ }^{1} S_{0}\right)\right| P_{Q}(n S)\right\rangle^{1 / m} \\
& \quad=C_{A} \frac{\left|R_{n 0}^{P}(0)\right|^{2}}{2 \pi}\left(1-\frac{E_{n 0}^{(0)}}{m} \frac{2 \mathcal{E}_{3}}{9}+\frac{2 \mathcal{E}_{3}^{(2, t)}}{3 m^{2}}+\frac{c_{F}^{2} \mathcal{B}_{1}}{m^{2}}\right) \\
& \left\langle V_{Q}(n S)\left|O_{\mathrm{EM}}\left({ }^{3} S_{1}\right)\right| V_{Q}(n S)\right\rangle^{1 / m} \\
& \quad=C_{A} \frac{\left|R_{n 0}^{V}(0)\right|^{2}}{2 \pi}\left(1-\frac{E_{n 0}^{(0)}}{m} \frac{2 \mathcal{E}_{3}}{9}+\frac{2 \mathcal{E}_{3}^{(2, \mathrm{EM})}}{3 m^{2}}+\frac{c_{F}^{2} \mathcal{B}_{1}}{3 m^{2}}\right),
\end{aligned}
$$

$$
\begin{aligned}
& \left\langle P_{Q}(n S)\left|O_{\mathrm{EM}}\left({ }^{1} S_{0}\right)\right| P_{Q}(n S)\right\rangle^{1 / m} \\
& \quad=C_{A} \frac{\left|R_{n 0}^{P}(0)\right|^{2}}{2 \pi}\left(1-\frac{E_{n 0}^{(0)}}{m} \frac{2 \mathcal{E}_{3}}{9}+\frac{2 \mathcal{E}_{3}^{(2, \mathrm{EM})}}{3 m^{2}}+\frac{c_{F}^{2} \mathcal{B}_{1}}{m^{2}}\right), \\
& \left\langle\chi_{Q}(n J S)\left|O_{1}\left({ }^{2 S+1} P_{J}\right)\right| \chi_{Q}(n J S)\right\rangle^{1 / m} \\
& \quad=\left\langle\chi_{Q}(n J S)\left|O_{\mathrm{EM}}\left({ }^{2 S+1} P_{J}\right)\right| \chi_{Q}(n J S)\right\rangle^{1 / m} \\
& =\frac{3}{2} \frac{C_{A}}{\pi}\left|R_{n 1}^{(0) \prime}(0)\right|^{2}, \\
& \left\langle V_{Q}(n S)\left|\mathcal{P}_{1}\left({ }^{3} S_{1}\right)\right| V_{Q}(n S)\right\rangle^{1 / m} \\
& \quad=\left\langle P_{Q}(n S)\left|\mathcal{P}_{1}\left({ }^{1} S_{0}\right)\right| P_{Q}(n S)\right\rangle^{1 / m} \\
& \quad=\left\langle V_{Q}(n S)\left|\mathcal{P}_{\mathrm{EM}}\left({ }^{3} S_{1}\right)\right| V_{Q}(n S)\right\rangle^{1 / m} \\
& \quad=\left\langle P_{Q}(n S)\left|\mathcal{P}_{\mathrm{EM}}\left({ }^{1} S_{0}\right)\right| P_{Q}(n S)\right\rangle^{1 / m} \\
& \quad=C_{A} \frac{\left|R_{n 0}^{(0)}(0)\right|^{2}}{2 \pi}\left(m E_{n 0}^{(0)}-\mathcal{E}_{1}\right),
\end{aligned}
$$

$$
\begin{aligned}
\left\langle V_{Q}\right. & \left.(n S)\left|O_{8}\left({ }^{3} S_{1}\right)\right| V_{Q}(n S)\right\rangle^{1 / m} \\
& =\left\langle P_{Q}(n S)\left|O_{8}\left({ }^{1} S_{0}\right)\right| P_{Q}(n S)\right\rangle^{1 / m} \\
& =C_{A} \frac{\left|R_{n 0}^{(0)}(0)\right|^{2}}{2 \pi}\left(-\frac{2\left(C_{A} / 2-C_{F}\right) \mathcal{E}_{3}^{(2)}}{3 m^{2}}\right),
\end{aligned}
$$

$$
\begin{aligned}
& \left\langle V_{Q}(n S)\left|O_{8}\left({ }^{1} S_{0}\right)\right| V_{Q}(n S)\right\rangle^{1 / m} \\
& =\frac{\left\langle P_{Q}(n S)\left|O_{8}\left({ }^{3} S_{1}\right)\right| P_{Q}(n S)\right\rangle^{1 / m}}{3} \\
& \quad=C_{A} \frac{\left|R_{n 0}^{(0)}(0)\right|^{2}}{2 \pi}\left(-\frac{\left(C_{A} / 2-C_{F}\right) c_{F}^{2} \mathcal{B}_{1}}{3 m^{2}}\right), \\
& \frac{\left\langle V_{Q}(n S)\left|O_{8}\left({ }^{3} P_{J}\right)\right| V_{Q}(n S)\right\rangle^{1 / m}}{2 J+1} \\
& =\frac{\left\langle P_{Q}(n S)\left|O_{8}\left({ }^{1} P_{1}\right)\right| P_{Q}(n S)\right\rangle^{1 / m}}{9} \\
& =C_{A} \frac{\left|R_{n 0}^{(0)}(0)\right|^{2}}{2 \pi}\left(-\frac{\left(C_{A} / 2-C_{F}\right) \mathcal{E}_{1}}{9}\right)
\end{aligned}
$$

$\left\langle\chi_{Q}(n J S)\left|O_{8}\left({ }^{1} S_{0}\right)\right| \chi_{Q}(n J S)\right\rangle^{1 / m}=\frac{T_{F}}{3} \frac{\left|R_{n 1}^{(0) \prime}(0)\right|^{2}}{\pi m^{2}} \mathcal{E}_{3}$,

where the radial part of the vector $S$-wave function is $R_{n 101} \equiv R_{n 0}^{V}$ and the radial part of the pseudoscalar $S$-wave function is $R_{n 000} \equiv R_{n 0}^{P}$. The quantity $R_{n 1}^{(0) \prime}$ is the derivative of the radial part of the LO $P$-wave function. Any other dimension- 6 and dimension- $8 S$-wave matrix elements are 0 at the order considered here.

The nonanalytic contributions in $1 / m$ to the NRQCD matrix elements have been calculated up to (once normalized to $\left.m^{0}\right) \mathcal{O}\left(p^{3} / m^{3} \times \Lambda_{\mathrm{QCD}} / m \times m \alpha_{s} / \sqrt{m \Lambda_{\mathrm{QCD}}}\right)$ for $S$-wave matrix elements (Brambilla, Pineda, et al., 2004): 


$$
\begin{aligned}
& \left\langle V_{Q}(n S)\left|O_{1}\left({ }^{3} S_{1}\right)\right| V_{Q}(n S)\right\rangle^{1 / \sqrt{m}} \\
& =\left\langle V_{Q}(n S)\left|O_{\mathrm{EM}}\left({ }^{3} S_{1}\right)\right| V_{Q}(n S)\right\rangle^{1 / \sqrt{m}} \\
& \quad=C_{A} \frac{\left|R_{n 0}^{V}(0)\right|^{2}}{2 \pi}\left(1+\frac{4\left(2 C_{F}+C_{A}\right)}{3 \Gamma(7 / 2)} \frac{\alpha_{s} \mathcal{E}_{5 / 2}^{E}}{m^{1 / 2}}\right), \\
& \left\langle P_{Q}(n S)\left|O_{1}\left({ }^{1} S_{0}\right)\right| P_{Q}(n S)\right\rangle^{1 / \sqrt{m}} \\
& =\left\langle P_{Q}(n S)\left|O_{\mathrm{EM}}\left({ }^{1} S_{0}\right)\right| P_{Q}(n S)\right\rangle^{1 / \sqrt{m}} \\
& \quad=C_{A} \frac{\left|R_{n 0}^{P}(0)\right|^{2}}{2 \pi}\left(1+\frac{4\left(2 C_{F}+C_{A}\right)}{3 \Gamma(7 / 2)} \frac{\alpha_{S} \mathcal{E}_{5 / 2}^{E}}{m^{1 / 2}}\right) .
\end{aligned}
$$

All other matrix elements receive contributions which are $\mathcal{O}\left(m \alpha_{s} / \sqrt{m \Lambda_{\mathrm{QCD}}}\right)$ suppressed, under the condition (282), with respect to those listed in Eqs. (313)-(322).

Some comments are in order. All matrix elements are factorized into a part that is the wave function at the origin and a combination of gluon-field correlators. The wave function carries the dependence on the state and flavor content of the decaying heavy quarkonium (apart from the residual dependence on $m$ and $n$ in the binding energy and on $m$ in the logarithms in $c_{F}$ ), while the correlators only depend on the low-energy properties of QCD and are in this sense universal. They may be calculated once and forever, by means of lattice simulations (D’Elia et al., 1997; Bali et al., 1998; Foster and Michael, 1999; Bali and Pineda, 2004), specific models of the QCD vacuum (Baker et al., 1998; Brambilla, 2000; Di Giacomo et al., 2002), or extracted from experimental data (Brambilla, Eiras, et al., 2002; see also Sec. VIII.D). We emphasize that the factorization holds only if $\Lambda_{\mathrm{QCD}}$ $\gg E$, otherwise it would not be possible to disentangle the heavy quarkonium, whose energy is $E$, from the nonperturbative gluons.

The factorization is also the reason for the reduction in the number of nonperturbative parameters in going from NRQCD to $\mathrm{pNRQCD.} \mathrm{In} \mathrm{pNRQCD}$ these are the wave functions and the gluon-field correlators. Among these only the wave functions depend on the specific heavy-quarkonium state that we are considering. As discussed at the beginning of the section, the wave function may be calculated, in principle, in terms of QCD quantities by solving the Schrödinger equation (308). At the order at which they are given, Eqs. (313)-(316) are sensitive to the difference between the pseudoscalar and the vector $S$-wave function. For the other $S$-wave operators, the difference is not important at the present level of accuracy. The reduction in the number of parameters is more evident if we consider ratios of matrix elements of hadronic operators and electromagnetic ones. The wave-function dependence drops out and we are left with a combination of a few universal gluon-field correlators. In Sec. VIII.D, we discuss the phenomenological relevance of this for the calculation of bottomonium and charmonium inclusive decay widths.

Finally, we recall that, apart from the matrix elements $\left\langle V_{Q}(n S)\left|O_{1}\left({ }^{3} S_{1}\right)\right| V_{Q}(n S)\right\rangle$ and $\left\langle P_{Q}(n S)\left|O_{1}\left({ }^{1} S_{0}\right)\right| P_{Q}(n S)\right\rangle$ that are affected at relative order $\Lambda_{\mathrm{QCD}} / m$ $\times m \alpha_{s} / \sqrt{m \Lambda_{\mathrm{QCD}}}$, all other matrix elements listed above receive nonanalytic contributions from the threemomentum scale $\sqrt{m \Lambda_{\mathrm{QCD}}}$ at relative order $m \alpha_{s} / \sqrt{m \Lambda_{\mathrm{QCD}}}$ with respect to the leading piece. It may turn out that these contributions are numerically important since the suppression factor $m \alpha_{s} / \sqrt{m \Lambda_{\mathrm{QCD}}}$ may not be that small. In this case it would be important to have the leading nonanalytic contributions for all matrix elements. As long as this is not the case, nonanalytic contributions give the dominant source of uncertainty for the factorization formulas (317)-(322).

We conclude by giving the explicit formulas in pNRQCD for the electromagnetic and inclusive decay widths of heavy quarkonium into light particles at the present level of knowledge. This means that $S$-wave decay widths are given up to and including $\mathcal{O}(\operatorname{Im} f$ $\left.\times p^{3} / m^{2} \times \Lambda_{\mathrm{QCD}} / m \times m \alpha_{s} / \sqrt{m \Lambda_{\mathrm{QCD}}}\right)$ and $P$-wave decay widths up to and including $\mathcal{O}\left(\operatorname{Im} f \times p^{5} / m^{4}\right)$ :

$$
\begin{aligned}
\Gamma\left(V_{Q}(n S) \rightarrow L H\right)= & \frac{C_{A}}{\pi} \frac{\left|R_{n 0}^{V}(0)\right|^{2}}{m^{2}} \operatorname{Im} f_{1}\left({ }^{3} S_{1}\right) \\
& \times\left(1+\frac{4\left(2 C_{F}+C_{A}\right)}{3 \Gamma(7 / 2)} \frac{\alpha_{s} \mathcal{E}_{5 / 2}^{E}}{m^{1 / 2}}\right),
\end{aligned}
$$

$$
\begin{aligned}
\Gamma\left(P_{Q}(n S) \rightarrow L H\right)= & \frac{C_{A}}{\pi} \frac{\left|R_{n 0}^{P}(0)\right|^{2}}{m^{2}} \operatorname{Im} f_{1}\left({ }^{1} S_{0}\right) \\
& \times\left(1+\frac{4\left(2 C_{F}+C_{A}\right)}{3 \Gamma(7 / 2)} \frac{\alpha_{s} \mathcal{E}_{5 / 2}^{E}}{m^{1 / 2}}\right),
\end{aligned}
$$

$$
\begin{aligned}
\Gamma\left(\chi_{Q}(n J S) \rightarrow L H\right)= & \frac{C_{A}}{\pi} \frac{\left|R_{n 1}^{(0) \prime}(0)\right|^{2}}{m^{4}}\left(3 \operatorname{Im} f_{1}\left({ }^{2 S+1} P_{J}\right)\right. \\
& \left.+\frac{2 T_{F}}{3 C_{A}} \operatorname{Im} f_{8}\left({ }^{2 S+1} \mathrm{~S}_{S}\right) \mathcal{E}_{3}\right), \\
\Gamma\left(V_{Q}(n S) \rightarrow e^{+} e^{-}\right)= & \frac{C_{A}}{\pi} \frac{\left|R_{n 0}^{V}(0)\right|^{2}}{m^{2}} \operatorname{Im} f_{e e}\left({ }^{3} S_{1}\right) \\
& \times\left(1+\frac{4\left(2 C_{F}+C_{A}\right)}{3 \Gamma(7 / 2)} \frac{\alpha_{s} \mathcal{E}_{5 / 2}^{E}}{m^{1 / 2}}\right),
\end{aligned}
$$

$$
\begin{aligned}
\Gamma\left(P_{Q}(n S) \rightarrow \gamma \gamma\right)= & \frac{C_{A}}{\pi} \frac{\left|R_{n 0}^{P}(0)\right|^{2}}{m^{2}} \operatorname{Im} f_{\gamma \gamma}\left({ }^{1} S_{0}\right) \\
& \times\left(1+\frac{4\left(2 C_{F}+C_{A}\right)}{3 \Gamma(7 / 2)} \frac{\alpha_{s} \mathcal{E}_{5 / 2}^{E}}{m^{1 / 2}}\right),
\end{aligned}
$$

$$
\Gamma\left(\chi_{Q}(n J 1) \rightarrow \gamma \gamma\right)=3 \frac{C_{A}}{\pi} \frac{\left|R_{n 1}^{(0) \prime}(0)\right|^{2}}{m^{4}} \operatorname{Im} f_{\gamma \gamma}\left({ }^{3} P_{J}\right)
$$

for $J=0,2$. 
TABLE III. Recent determinations of $\bar{m}_{b}$ and $\bar{m}_{c}$ in the $\overline{\mathrm{MS}}$ scheme from the $\Upsilon(1 S)$ and $J / \psi(1 S)$ masses.

\begin{tabular}{ccc}
\hline \hline Reference & Order & $\bar{m}_{b}\left(\bar{m}_{b}\right)(\mathrm{GeV})$ \\
\hline Beneke and Signer, 1996 & NNLO & $4.24 \pm 0.09$ \\
Hoang, 1999 & NNLO & $4.21 \pm 0.09$ \\
Pineda, 2001 & NNLO & $4.210 \pm 0.090 \pm 0.025$ \\
Brambilla, Sumino, & NNLO & $4.190 \pm 0.020 \pm 0.025$ \\
and Vairo, 2002 & & \\
Penin and Steinhauser, 2002 & NNNLO & $4.349 \pm 0.070$ \\
Lee, 2003a & NNNLO & $4.20 \pm 0.04$ \\
Contreras et al., 2004 & NNNLO & $4.241 \pm 0.070$ \\
\hline Reference & Order & $\bar{m}_{c}\left(\bar{m}_{c}\right)(\mathrm{GeV})$ \\
\hline Brambilla, Sumino, & NNLO & $1.24 \pm 0.020$ \\
and Vairo, 2001 & & \\
\hline \hline
\end{tabular}

\section{PHENOMENOLOGICAL APPLICATIONS}

\section{A. Determinations of $m_{b}$ and $m_{c}$ from the $1 S$ resonances}

Here we present state-of-the-art determinations of the bottom and charm masses from the ground-state bottomonium and charmonium masses.

For precise determinations of those parameters, we need a situation where the dynamics can be described by a weak-coupling analysis (at least in a first approximation) and where nonperturbative effects are small. Therefore the first question we should answer is are we in such a dynamical situation? For the bottomonium and charmonium systems, we believe that the masses $m_{b}$ and $m_{c}$ are much larger than $\Lambda_{\mathrm{QCD}}$. This is not enough, however, since we also need $m v \gg \Lambda_{\mathrm{QCD}}$. If this is the case then we are dealing (in a first approximation) with a Coulomb-type bound state. In this situation we can apply the results of Sec. IV.G once the renormalon cancellation along the lines of Sec. V has been used. In other words, our starting point will be Eq. (194). Let us see whether the assumption $m v \gg \Lambda_{\mathrm{QCD}}$ is reasonable for bottomonium and charmonium ground states. The momentum transfer in the first case is around $\lesssim 2 \mathrm{GeV}$ whereas in the second case it is around $\lesssim 1 \mathrm{GeV}$. The momentum transfer between the heavy quark and antiquark lies in the deep Euclidean domain. Therefore the computation does not rely on local duality (at least to low orders in perturbation theory). The assumption $m v$ $\gg \Lambda_{\mathrm{QCD}}$ then becomes equivalent to believing in perturbative calculations in the Euclidean domain in the above range of energies. We report on work in which this assumption is taken for the bottomonium as well as for the charmonium ground state. The relative size between the US scale and $\Lambda_{\mathrm{QCD}}$ remains to be fixed.

Let us now consider recent determinations available in the literature, which we cite in Table III. In the first three references, as well as in the article by Penin and Steinhauser (2002), finite charm-mass effects due to the potential and self-energy, calculated by Gray et al.
(1990); Eiras and Soto (2000); Hoang (2000); Melles (2000), were not included. In all the references except Beneke and Signer (1999) (at the moment of that computation the conversion from the pole to the MS masses was not known with the required accuracy), the conversion from the threshold (or pole) masses to the MS has been performed to three loops. The NNNLO analyses should be understood only as almost complete, since the three-loop static-potential coefficient was only estimated. In the work of Beneke and Signer (1999), a NNLO analysis was done in the PS scheme. In that of Hoang (1999), a NNLO analysis was done in the $1 S$ scheme. In Pineda's work (2001), a NNLO analysis was done in the RS scheme as well as an analysis at NNNLO including the logarithms at this order and the large- $\beta_{0}$ result. In Brambilla, Sumino, and Vairo (2002), a NNLO analysis was done in the MS scheme using the upsilon expansion. Penin and Steinhauser (2002) used a NNNLO analysis in the on-shell scheme. We believe that the difference with respect to the other results is due to the presence of the renormalon, as well as the way US and nonperturbative effects were implemented since the authors assume $m v^{2} \gg \Lambda_{\mathrm{QCD}}$. In the work of Lee (2003a) a NNNLO analysis was done in a scheme similar to the RS one. He included the US contribution within perturbation theory. Contreras et al. (2004) also used a NNNLO analysis in a scheme similar to the RS one. In this case, the US contribution was also treated perturbatively but in a different way from the soft one. It would be extremely interesting to repeat these analyses without the US contribution. Actually, in the Contreras et al. (2004) analysis, it is easy to separate out the US contribution (although it is not fully clear in which scheme). If one eliminates the US contribution in this case, the bottom mass goes down by around $50 \mathrm{MeV}$ leading to good agreement with previous analyses. Nevertheless, it remains to be seen what would happen (Lee, 2003a) if a similar approach were applied.

We would also like to mention the determination of the charm mass from the $J / \psi(1 S)$ mass (Brambilla, Sumino, and Vairo, 2001). The authors perform a complete NNLO analysis in the $1 S$ scheme. It would be very interesting to perform a similar analysis with a different threshold mass, as well as to do the NNNLO analysis in order to see whether the result remains stable.

In the above analyses, with the exception of that of Penin and Steinhauser (2002), the nonperturbative effects have been left unevaluated. In some cases the nonperturbative results obtained in the limit $m v^{2} \gg \Lambda_{\mathrm{QCD}}$ have been used to estimate their size.

The main sources of errors and possible improvements are the following. None of the above analyses has yet incorporated the resummation of logarithms available at NNLLO. It would be interesting to see its effect on the mass of the heavy quarkonium. So far all (almost complete) NNNLO evaluations have been done assuming that the US contribution can be computed within perturbation theory. It would be most interesting to perform the NNNLO analysis without the US piece. Two of the (potentially) major sources of errors in these kinds of 
evaluations of the heavy-quarkonium mass are the nonperturbative contribution (140) and possible effects due to subleading renormalons [see the discussion by Pineda (2001)]. Any reliable determination of Eq. (140) will have an immediate impact on our understanding of the theoretical errors. On the one hand, it would put on more solid ground our implicit assumption that the LO solution corresponds to a Coulomb-type bound state. Once this is achieved, it would bring the error estimates of the nonperturbative effects from a qualitative level to a quantitative one, (hopefully) decreasing their size significantly. On the other hand, one may think of crosschecking these results with other determinations. The fact that the difference happens to be relatively small supports the belief that (perturbative and nonperturbative) higher-order effects are indeed not very large. In order to have an independent handle on the size of the nonperturbative corrections, one may consider the difference between the lattice simulation of the static potential and the perturbative prediction (Pineda, 2003b). If one neglects possible effects of unquenching, one gets (in the static limit) nonperturbative contributions, which are not larger than $\sim 100 \mathrm{MeV}$. A precise determination would require an accurate determination of the chromoelectric correlator which appears in Eq. (140) from the lattice. In this respect, we note that by using the data on the gluelump masses reported in Table II one obtains $\Lambda_{E} \simeq 1.25 \mathrm{GeV}$, which is much larger than the US scale. This may indicate that the actual situation, even for the ground-state bottomonium, is $m v \gg \Lambda_{\mathrm{QCD}} \gg m v^{2}$, at least as far as the computation of Eq. (140) is concerned. Then the results of Sec. VII.F would apply.

\section{B. Spectroscopy in the weak-coupling regime}

Along the lines of the previous section, once it is assumed that the $Y(1 S)$ can be described by the weakcoupling version of $\mathrm{pNRQCD}$, it should be possible to give a prediction for the $\eta_{b}(1 S)$ mass. If this belief is extended to the $J / \psi(1 S)$, it should also be possible to give predictions for the $B_{c}(1 S)^{25}$ and $B_{c}(1 S)^{*}$ masses, as well as to check the theory by comparing them with the experimental value of the $\eta_{c}(1 S)$ mass.

Working in the $1 S$ scheme at NNLO, Brambilla and Vairo (2000a) obtained a prediction for the $B_{c}(1 S)$ mass:

$$
M\left(B_{c}\right)=6326_{-9}^{+29} \mathrm{MeV},
$$

where the error accounts only for higher-order perturbative corrections and uncertainties in $\alpha_{s}\left(M_{Z}\right)$. The error due to nonperturbative contributions has been estimated to be $40-100 \mathrm{MeV}$. It is argued there that the nonperturbative contributions to the $B_{c}$ mass in the $1 S$-mass scheme come out as the following combination of nonperturbative contributions in the pole-

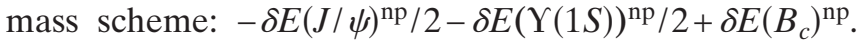

\footnotetext{
${ }^{25}$ Although its mass has been measured to be $6.40 \pm 0.39 \pm 0.13 \mathrm{GeV}$ (Abe et al., 1998), the precision is not good enough to test the theory.
}

Therefore cancellations may occur if all three corrections are of the same type and size. This may substantially reduce the total size of the nonperturbative corrections to the $B_{c}$ in the $1 S$-mass scheme. Brambilla, Sumino, and Vairo (2001) made a similar determination using the MS $c$ and $b$ masses. The result is very similar: $M\left(B_{c}\right)=6324 \pm 23 \mathrm{MeV}$. Again the error only accounts for higher-order perturbative corrections and uncertainties in $\alpha_{s}\left(M_{Z}\right)$. The error due to nonperturbative contributions has not been estimated there. Brambilla, Sumino, and Vairo (2002) also included charm-mass effects in the analysis. They lower slightly the central value: $M\left(B_{c}\right)=6309 \pm 17 \mathrm{MeV}$. The error is as above.

In the case of bottomonium, Kniehl et al. (2004) calculated the hyperfine splitting of the ground state at NLLO in the on-shell scheme (the effects due to the pole-mass renormalon are subleading). For this observable, the resummation of the logarithms along the lines discussed in Sec. IV.H seems important. The authors have given a rather precise prediction for the mass of the $\eta_{b}(1 S)$, which uses the experimental value of $M_{\Upsilon(1 S)}$,

$$
M\left(\eta_{b}(1 S)\right)=9421 \pm 11(\text { th })_{-8}^{+9}\left(\delta \alpha_{s}\right) \mathrm{MeV},
$$

where the errors due to the higher-order perturbative corrections and the nonperturbative effects are added up in quadrature in "th," whereas " $\delta \alpha_{s}$ " stands for the uncertainty in $\alpha_{s}\left(M_{Z}\right)=0.118 \pm 0.003$. They also obtained a value for the charmonium ground-state hyperfine splitting, $M(J / \psi(1 S))-M\left(\eta_{c}(1 S)\right) \simeq 104 \mathrm{MeV}$, to be compared with the experimental value of $117.7 \mathrm{MeV}$. Recksiegel and Sumino (2004) have performed a numerical NLO analysis of these hyperfine splittings. For bottomonium they get $\simeq 44 \mathrm{MeV}$, which compares well with the above number, and for charmonium $\simeq 88 \mathrm{MeV}$, which is somewhat lower.

Penin et al. (2004a) have also calculated the hyperfine splitting of the $B_{c}$ ground state at NLLO in a way similar to that of Kniehl et al. (2004). They obtain

$$
M\left(B_{c}^{*}\right)-M\left(B_{c}\right)=65 \pm 24(\mathrm{th})_{-16}^{+19}\left(\delta \alpha_{s}\right) \mathrm{MeV}
$$

where the errors are as in Eq. (332). This result, combined with Eq. (331), or, eventually, with a more accurate experimental determination of the $B_{c}$ mass, provides a prediction for the $B_{c}^{*}$ mass.

Brambilla, Sumino, and Vairo $(2001,2002)$ considered higher excitations of the bottomonium system at NNLO in the MS mass scheme using the upsilon expansion (the latter reference also included finite charm-mass effects). It is not obvious a priori that these can be described under the kinematical assumption $m v \gg \Lambda_{\mathrm{QCD}}$, however, it is worth investigating this possibility. The results for the levels that turn out to be stable in this analysis are shown in Table IV. We note that at least a part of the higher bottomonium levels seems to be reasonably well described in perturbation theory. In particular, the equal level spacing, characteristic for the quarkonium spectrum, is reasonably well reproduced without making use of a confining potential. This behavior seems to originate from self-energy contribution remnants of the 
TABLE IV. Comparison of the theoretical predictions of some of the bottomonium levels obtained by Brambilla, Sumino, and Vairo (2002) with the experimental data. The errors come from summing quadratically uncertainties in $\alpha_{s}$, higher-order corrections, and finite charm-mass corrections (Brambilla et al., 2004).

\begin{tabular}{ccc}
\hline \hline$\Upsilon$ & $M(\Upsilon)^{\exp }(\mathrm{MeV})$ & $M(\mathrm{Y})(\mathrm{MeV})$ \\
\hline$\Upsilon\left(1{ }^{3} P_{0}\right)$ & 9860 & $9995(83)$ \\
$\Upsilon\left(1{ }^{3} P_{1}\right)$ & 9893 & $10004(86)$ \\
$\Upsilon\left(1{ }^{3} P_{2}\right)$ & 9913 & $10012(89)$ \\
$\Upsilon\left(2{ }^{3} S_{1}\right)$ & 10023 & $10084(102)$ \\
$\Upsilon\left(1{ }^{3} P_{0}\right)$ & 10232 & $10548(239)$ \\
$\Upsilon\left(1{ }^{3} P_{1}\right)$ & 10255 & $10564(247)$ \\
$\Upsilon\left(1{ }^{3} P_{2}\right)$ & 10269 & $10578(258)$ \\
$\Upsilon\left(3{ }^{3} S_{1}\right)$ & 10355 & $10645(298)$ \\
\hline \hline
\end{tabular}

renormalon cancellation and may reflect, from the point of view of the spectrum, the numerical agreement mentioned in Sec. VI that is found in some situations between the perturbative static potential and the lattice data up to very large distances. Indeed, it was this phenomenological analysis that triggered part of the subsequent analysis of the static potential measured on the lattice in terms of perturbative QCD. Moreover, since for higher levels the experimental data agree with the theoretical results within the uncertainties, we may expect this to be the case also for the bottomonium ground state, suggesting very small nonperturbative corrections to it. Along similar lines, but within a numerical analysis, fine splittings of bottomonium and charmonium levels have been considered at NLO by Recksiegel and Sumino (2004).

\section{Electromagnetic inclusive decay widths in the weak- coupling regime}

The electromagnetic inclusive decay widths are known at NNLO (see Sec. IV.G). Nevertheless, they suffer from large-scale uncertainties, which have so far prevented their use in phenomenological analysis. This also affects the accuracy of sum rules (see the discussion in Sec. VIII.E).

Recently, there have been a few phenomenological analyses including the resummation of logarithms (see Sec. IV.H). The impact of these logarithms appears to be large and the overall convergence of the series seems to improve. For bottomonium, Penin et al. (2004b) considered the complete result with NNLLO accuracy for the ratio of the spin- 1 and spin- 0 production in the on-shell scheme (at this order effects due to the pole-mass renormalon are subleading). The logarithmic expansion shows nice convergence and stability [see Fig. 19(a)] despite the presence of US contributions with $\alpha_{s}$ evaluated at a rather low scale $\nu^{2} / m_{b}$. At the same time, the perturbative corrections are important and reduce the LO result by approximately $40 \%$. For illustration, at the scale of
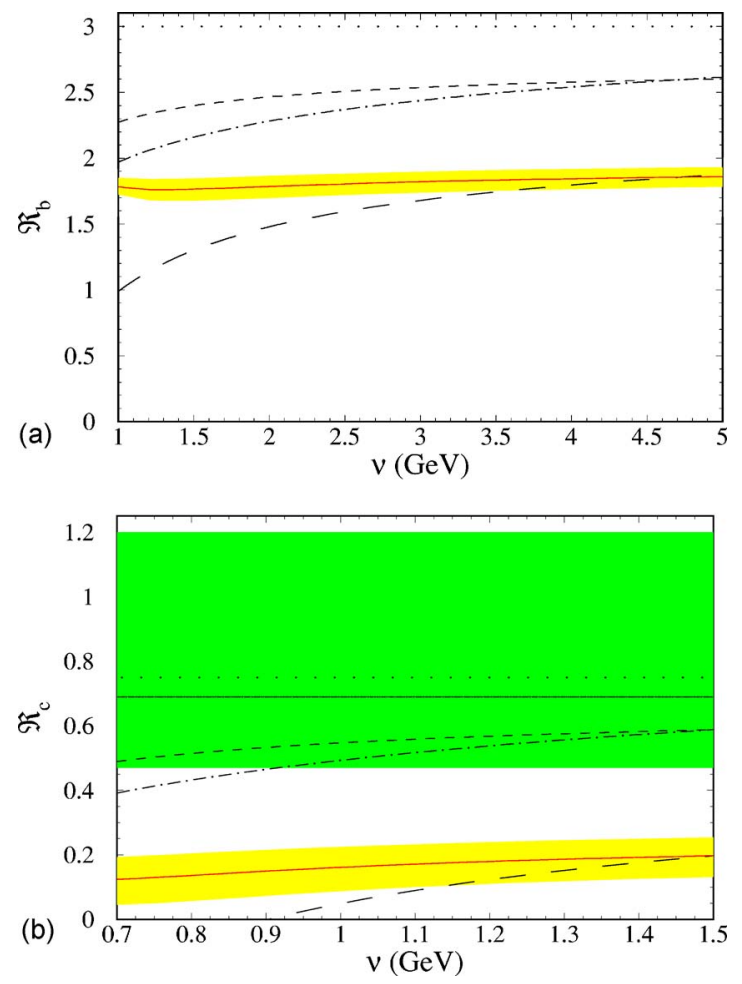

FIG. 19. (Color online) The spin ratio as a function of the renormalization scale $\nu$ in $\mathrm{LO} \equiv \mathrm{LL}$ (dotted line), NLO (shortdashed line), NNLO (long-dashed line), NLL (dot-dashed line), and NNLL (solid line) approximation. For the NNLL result the band reflects the errors due to $\alpha_{s}\left(M_{Z}\right)=0.118 \pm 0.003$. (a) The bottomonium ground-state case for which $\nu_{h}=m_{b}$. (b) The charmonium ground-state case for which $\nu_{h}=m_{c}$. In the charmonium case, the upper band represents the experimental error of the ratio (Eidelman et al., 2004), where the central value is given by the horizontal solid line. From Penin et al., 2004b.

minimal sensitivity, $\nu=1.295 \mathrm{GeV}$, one has the following series:

$$
\mathcal{R}_{b} \equiv \frac{\Gamma\left(\Upsilon(1 S) \rightarrow e^{+} e^{-}\right)}{\Gamma\left(\eta_{b}(1 S) \rightarrow \gamma \gamma\right)}=\frac{1}{3 e_{b}^{2}}(1-0.302-0.111)
$$

In contrast, the fixed-order expansion blows up at the scale of the inverse Bohr radius. Nonperturbative effects contribute in the next-to-next-to-next-to-next-toleading-logarithmic approximation, which is far beyond the precision of this computation. Note that the nonperturbative contribution to the ratio of decay rates is suppressed by a factor $v^{2}$ in comparison to the binding energy and decay rates, where the leading nonperturbative effect is due to chromoelectric dipole interaction. Thus by using the available experimental data on the $Y$ meson as input, one can predict the production and annihilation rates of the yet undiscovered $\eta_{b}$ meson. In particular, one can predict the $\eta_{b}(1 S)$ decay rate using the experimental value for the $\mathrm{Y}(1 S)$ decay rate (Penin et al., 2004b): 


$$
\begin{aligned}
& \Gamma\left(\eta_{b}(1 S) \rightarrow \gamma \gamma\right) \\
& \quad=0.659 \pm 0.089(\mathrm{th})_{-0.018}^{+0.019}\left(\delta \alpha_{s}\right) \pm 0.015(\text { expt }) \mathrm{keV}
\end{aligned}
$$

where $\nu=1.295 \mathrm{GeV}$ was taken as the central value, the difference between the NLLO and NNLLO result for the theoretical error, and $\alpha_{s}\left(M_{Z}\right)=0.118 \pm 0.003$. The last error in Eq. (335) reflects the experimental error of $\Gamma\left(Y(1 S) \rightarrow e^{+} e^{-}\right)=1.314 \pm 0.029 \mathrm{keV}$ (Eidelman et al., 2004). This value considerably exceeds the result for the absolute value of the decay width obtained by Pineda (2003a) on the basis of a full NLLO analysis including the spin-independent part: $\quad \Gamma\left(\eta_{b}(1 S) \rightarrow \gamma \gamma\right)$ $=0.35 \pm 0.1(\mathrm{th}) \pm 0.05\left(\delta \alpha_{s}\right) \mathrm{keV}$. This can be a signal of slow convergence of the logarithmic expansion for the spin-independent contribution, which is more sensitive to the dynamics of the bound state and in particular to the US contribution, as has been discussed above. On the other hand, renormalon effects (Braaten and Chen, 1998; Bodwin and Chen, 1999) could produce some systematic errors in the purely perturbative evaluations of the production or annihilation rates. The problem is expected to be more severe for the charmonium case discussed below.

We would like to point out that the one-loop result for $\nu=m_{b}$ overshoots the NNLLO result by approximately $30 \%$. This casts some doubts on the accuracy of the existing $\alpha_{s}$ determination from the $\Gamma(Y$ $\rightarrow$ light hadrons $) / \Gamma\left(\Upsilon \rightarrow e^{+} e^{-}\right)$decay rate ratio, which gives $\alpha_{s}\left(m_{b}\right)=0.177 \pm 0.01$, well below the "world average" value (Eidelman et al., 2004). The theoretical uncertainty in the analysis is estimated through the scale dependence of the one-loop result. The analysis of the photon-mediated annihilation rates indicates that the actual magnitude of the higher-order corrections is most likely quite far beyond such an estimate and the theoretical uncertainty given by Eidelman et al. (2004) should be increased by a factor of 2 . This brings the result for $\alpha_{s}$ into a $1 \sigma$ distance from the world average value.

For charmonium, the same analysis was performed by Penin et al. (2004b). The NNLO approximation becomes negative at an intermediate scale between $\alpha_{s} m_{c}$ and $m_{c}$ [see Fig. 19(b)] and the use of the RG is mandatory in order to get a sensible perturbative approximation. The NNLLO approximation has good stability against the scale variation but the logarithmic expansion does not converge well. This is the main factor that limits the theoretical accuracy since the nonperturbative contribution is expected to be under control. For illustration, at the scale of minimal sensitivity, $\nu=0.645 \mathrm{GeV}$, one obtains

$$
\mathcal{R}_{c} \equiv \frac{\Gamma\left(J / \psi(1 S) \rightarrow e^{+} e^{-}\right)}{\Gamma\left(\eta_{c}(1 S) \rightarrow \gamma \gamma\right)}=\frac{1}{3 e_{c}^{2}}(1-0.513-0.326)
$$

The central value is $2 \sigma$ below the experimental one. The discrepancy may be explained by large higher-order contributions. This should not be surprising because of the rather large value of $\alpha_{s}$ at the inverse Bohr radius of charmonium. For the charmonium hyperfine splitting, however, the logarithmic expansion converges well and the prediction of the $\mathrm{RG}$ is in agreement with the experimental data. One can try to improve the convergence of the series for the production or annihilation rates by accurately taking into account the renormalonrelated contributions. One point to note is that with a potential-model evaluation of the wave-function correction the sign of the NNLO term is reversed in the charmonium case (Czarnecki and Melnikov, 2001). At the same time the subtraction of the pole-mass renormalon from the perturbative static potential makes explicit that the potential is steeper and closer to lattice results and to phenomenological potential models, as we have seen in Sec. VI. Therefore the incorporation of higher-order effects from the static potential may improve the agreement with experiment. Finally, we mention that a NLLO evaluation for the $\eta_{c}(1 S) \rightarrow \gamma \gamma$ decay reproduces in the minimal sensitivity region the experimental value (Pineda, 2003a).

\section{Inclusive decay widths in the strong-coupling regime}

At the end of Sec. II, we pointed out that the application of the NRQCD factorization formulas to inclusive annihilation widths of quarkonium was somehow limited by the large number and poor knowledge of the NRQCD four-fermion matrix elements. The pNRQCD factorization formulas presented in Sec. VII.H make both problems less severe by reducing the number of nonperturbative parameters and by factorizing the wave-function dependence. As a consequence, for systems to which it may be applied, pNRQCD in the strong-coupling regime has more predictive power than NRQCD. In the following, we present some of the predictions that are specific to pNRQCD. We remark that the problem of the poor convergence of the perturbative series for the NRQCD matching coefficients, also pointed out at the end of Sec. II, is specific to the hardscale factorization and will persist at the level of $\mathrm{pN}$ RQCD.

Let us consider the following ratios of hadronic and electromagnetic annihilation widths for states with the same principal quantum number $(J=0,2)$ :

$$
\begin{aligned}
& R_{n}^{V}=\frac{\Gamma\left(V_{Q}(n S) \rightarrow L H\right)}{\Gamma\left(V_{Q}(n S) \rightarrow e^{+} e^{-}\right)}, \\
& R_{n}^{P}=\frac{\Gamma\left(P_{Q}(n S) \rightarrow L H\right)}{\Gamma\left(P_{Q}(n S) \rightarrow \gamma \gamma\right)}, \quad R_{n}^{\chi}=\frac{\Gamma\left(\chi_{Q}(n J 1) \rightarrow L H\right)}{\Gamma\left(\chi_{Q}(n J 1) \rightarrow \gamma \gamma\right)} .
\end{aligned}
$$

It is a specific prediction of $\mathrm{pNRQCD}$ that for states for which the assumption $\Lambda_{\mathrm{QCD}} \gg E$ holds, the wavefunction dependence drops out of the right-hand side of the above equations. The residual flavor dependence is encoded in the powers of $1 / m$, in $E_{n 0}^{(0)}$, and in the Wilson coefficients, while the residual dependence on the principal quantum number is encoded in the LO binding 
energy $E_{n 0}^{(0)}$. The Wilson coefficients may be calculated in perturbation theory and the binding energy may be derived from the quarkonium mass $M(n S): M(n S)-2 m$ $\simeq E_{n 0}^{(0)}$. The only unknown quantities are the gluon-field correlators. The crucial point is that these do not depend on the flavor and the quarkonium quantum numbers. Therefore on the whole set of quarkonium states for which the pNRQCD formulas apply the number of nonperturbative parameters has decreased with respect to NRQCD. As discussed in Sec. VII.H, the gluon-field correlators may be extracted either from lattice simulations or specific models of the QCD vacuum or from experimental data. We shall come back to this last possibility at the end of the section.

Here we consider combinations of ratios in which even the dependence on the correlators drops out and predictions based purely on perturbative QCD are possible. Let us consider the ratios between $R_{n}^{V}$ and $R_{n}^{P}$ with different principal quantum numbers at order $E / m$. Contributions coming from the nonanalytic scale $\sqrt{m \Lambda_{\mathrm{QCD}}}$ have not been calculated to that order, however, they appear to be suppressed in the ratio. We obtain

$\frac{R_{n}^{V}}{R_{m}^{V}}=1+\left(\frac{\operatorname{Im} g_{1}\left({ }^{3} S_{1}\right)}{\operatorname{Im} f_{1}\left({ }^{3} S_{1}\right)}-\frac{\operatorname{Im} g_{e e}\left({ }^{3} S_{1}\right)}{\operatorname{Im} f_{e e}\left({ }^{3} S_{1}\right)}\right) \frac{M(n S)-M(m S)}{m}$,

$\frac{R_{n}^{P}}{R_{m}^{P}}=1+\left(\frac{\operatorname{Im} g_{1}\left({ }^{1} S_{0}\right)}{\operatorname{Im} f_{1}\left({ }^{1} S_{0}\right)}-\frac{\operatorname{Im} g_{\gamma \gamma}\left({ }^{1} S_{0}\right)}{\operatorname{Im} f_{\gamma \gamma}\left({ }^{1} S_{0}\right)}\right) \frac{M(n S)-M(m S)}{m}$.

Due to the pNRQCD factorization, the octet-type contributions cancel in the ratio, differently from what is predicted in NRQCD within the standard power counting (Gremm and Kapustin, 1997). In the vector case we get for the $\Upsilon(2 S)$ and $\Upsilon(3 S)$ state $\left(m_{b} \simeq 5 \mathrm{GeV}\right) R_{2}^{\Upsilon} / R_{3}^{\Upsilon}$ $\simeq 1.3$, which is close to the experimental central value of about 1.4 from Eidelman et al. (2004). In the pseudoscalar case, since $\operatorname{Im} g_{1}\left({ }^{1} S_{0}\right) / \operatorname{Im} f_{1}\left({ }^{1} S_{0}\right)$ - $\operatorname{Im} g_{\gamma \gamma}\left({ }^{1} S_{0}\right) / \operatorname{Im} f_{\gamma \gamma}\left({ }^{1} S_{0}\right)$ is of $\mathcal{O}\left(\alpha_{s}\right)$, we find that, at order $E / m, R_{n}^{P}$ is the same for all radial excitations.

As mentioned above, it is possible to fix the gluonfield correlators on some experimental set of data and use them on some other. For instance, one may extract them from charmonium data and calculate bottomonium widths. This is particularly useful since at present, bottomonium data are less abundant than charmonium ones. The program has been carried out for $P$-wave decays by Brambilla, Eiras, et al. (2002). These depend on just one correlator, $\mathcal{E}_{3}$, which may be extracted from $P$-wave charmonium decay data. The result is shown in Fig. 20. At the scale of $1 \mathrm{GeV}$ one finds

$$
\mathcal{E}_{3}(1 \mathrm{GeV})=5.3_{-2.2}^{+3.5}(\mathrm{expt})
$$

where errors refer only to experimental uncertainties on the charmonium decay widths (in particular, uncertainties related to higher orders in the perturbative series,

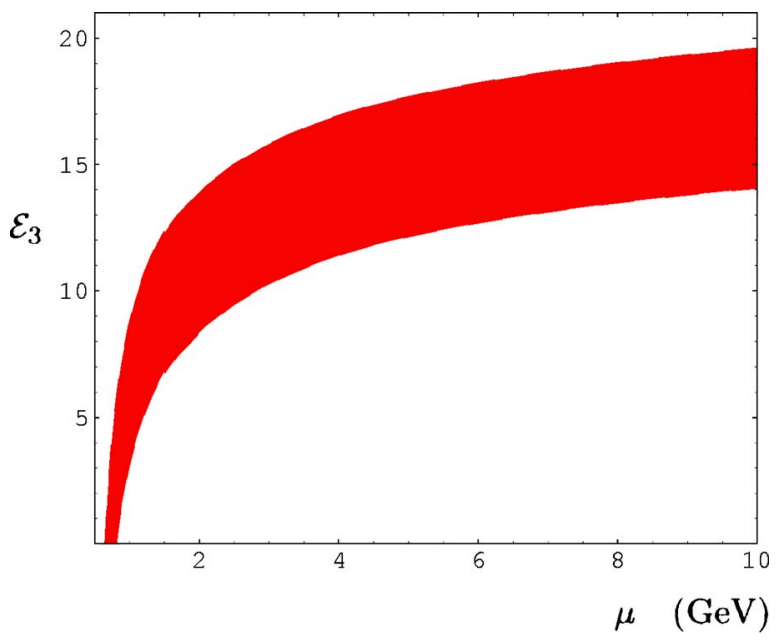

FIG. 20. (Color online) Plot of the one-loop RG-improved expression for $\mathcal{E}$ vs $\mu: \mathcal{E}(\mu)=\mathcal{E}(m)$ $+\left(24 N_{c} C_{F} / \beta_{0}\right) \ln \left[\alpha_{s}(m) / \alpha_{s}(\mu)\right]$. $\mathcal{E}(m)$ has been extracted from charmonium $P$-wave data. The error band accounts only for the uncertainties inherited from the charmonium data. From Vairo, 2003.

which may be potentially large, have not been included). In any case, the given value is compatible with the values that are usually assigned to the NRQCD octet and singlet matrix elements [e.g., from the fit given by Maltoni (2000) one obtains $\left.\mathcal{E}_{3}(1 \mathrm{GeV})=3.6_{-2.9}^{+3.6}(\mathrm{expt})\right]$, while the bottomonium lattice data given by Bodwin et al. $(1996,2002)$ appear to give a lower value. Once $\mathcal{E}_{3}$ is known it may be inserted into Eqs. (327) and (330) to get the ratios of annihilation widths of bottomonium $P$ waves. In practice, in $\mathrm{pNRQCD}$ at the order at which Eqs. (327) and (330) are valid, the $12 P$-wave bottomonium and charmonium states that lie below threshold depend on 4 nonperturbative parameters ( 3 wave functions +1 chromoelectric correlator $\mathcal{E}_{3}$ ). The reduction of the number of unknown nonperturbative parameters by 2 with respect to NRQCD allows one to formulate two specific new predictions of pNRQCD:

$$
\frac{\Gamma\left(\chi_{b 0}(1 P) \rightarrow L H\right)}{\Gamma\left(\chi_{b 1}(1 P) \rightarrow L H\right)}=\frac{\Gamma\left(\chi_{b 0}(2 P) \rightarrow L H\right)}{\Gamma\left(\chi_{b 1}(2 P) \rightarrow L H\right)}=8.0 \pm 1.3,
$$

or alternatively

$$
\frac{\Gamma\left(\chi_{b 1}(1 P) \rightarrow L H\right)}{\Gamma\left(\chi_{b 2}(1 P) \rightarrow L H\right)}=\frac{\Gamma\left(\chi_{b 1}(2 P) \rightarrow L H\right)}{\Gamma\left(\chi_{b 2}(2 P) \rightarrow L H\right)}=0.50_{-0.04}^{+0.06},
$$

where $\mathcal{E}_{3}$ is taken from Fig. 20 and the NRQCD matching coefficients are taken at NLO. The errors refer only to the uncertainty in $\mathcal{E}_{3}$. In Fig. 21 we plot the above ratios as functions of the factorization scale $\mu$. We note that the scale dependence of $\mathcal{E}_{3}$ (see Fig. 20) has been smoothed out in the plots of Fig. 21, as expected in a physical quantity [compare the cancellation of the leading-order IR divergences between the singlet matching coefficients and the octet matrix elements discussed 

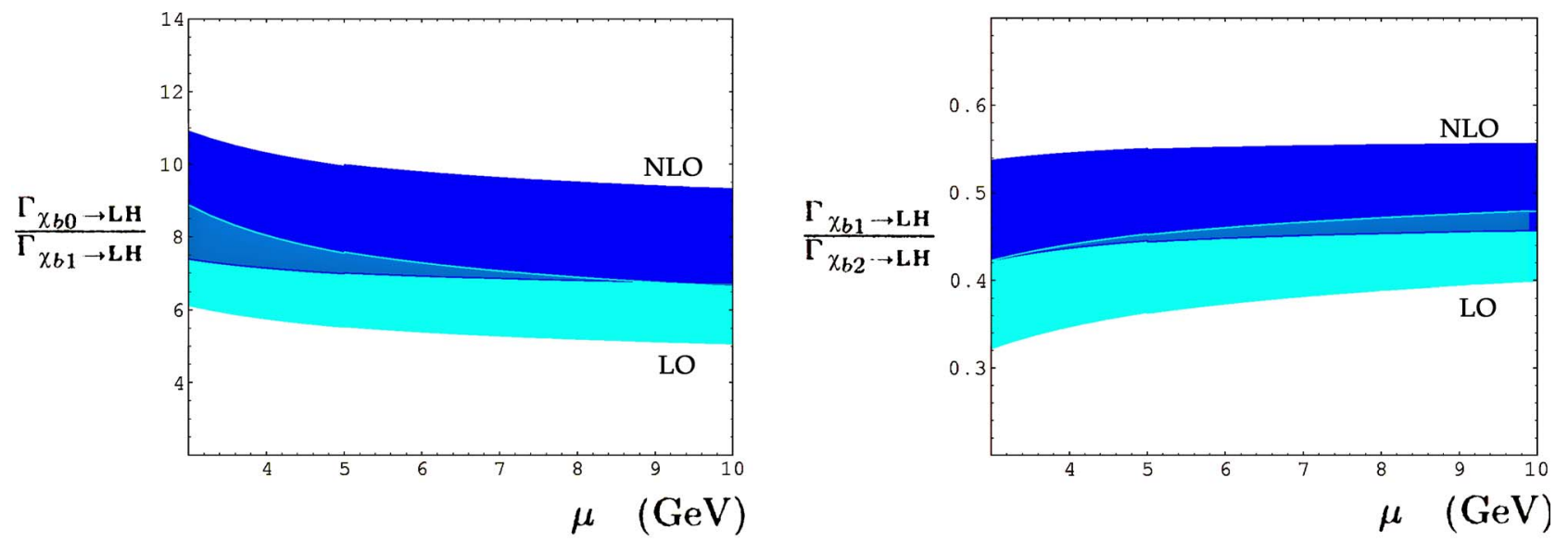

FIG. 21. (Color online) The left-hand side of Eqs. (341) and (342) plotted vs $\mu$. We have taken $\mathcal{E}_{3}$ from Fig. 20. The LO and NLO bands refer to the Wilson coefficients at LO and NLO, respectively. From Vairo, 2003.

in the paragraph after Eq. (47)]. The large NLO corrections are reflected by the extension of the nonoverlapping regions in the two bands in Fig. 21. Recent CLEO measurements give [see Cinabro et al. (2002), corrected by Brambilla et al. (2004)] $\Gamma\left(\chi_{b 0}(2 P) \rightarrow L H\right) / \Gamma\left(\chi_{b 2}(2 P)\right.$ $\rightarrow L H)=6.1 \pm 2.8$, which agree inside the large errors with the above predictions, and $\Gamma\left(\chi_{b 1}(2 P)\right.$ $\rightarrow L H) / \Gamma\left(\chi_{b 2}(2 P) \rightarrow L H\right)=0.25 \pm 0.09$, which is somewhat lower than above.

The above approach may eventually be extended to a global fit of all correlators appearing in $S$ - and $P$-wave annihilation widths. The obtained values could then be used to predict annihilation ratios of quarkonium states that are unknown or to improve present determinations. This program still requires the calculation of the contribution coming from the nonanalytic scale $\sqrt{m \Lambda_{\mathrm{QCD}}}$, at least at relative order $E / m$ and $\Lambda_{\mathrm{QCD}}^{2} / m^{2}$, for $S$ waves (that is with the same accuracy as the contributions coming from the analytic scales listed in Sec. VII.H) and the resummation of large contributions in the perturbative series of the four-fermion matching coefficients.

\section{E. Nonrelativistic sum rules}

NR sum rules are a classical example for the application of NR EFTs and the determination of the heavyquark masses such as charm and bottom. The key point is the relation between $\Pi\left(q^{2}\right)$ at $q^{2}=0$ to moments of the total cross section $\sigma\left(e^{+} e^{-} \rightarrow Q \bar{Q}\right)$. $\Pi\left(q^{2}\right)$ is defined in terms of the correlator of two electromagnetic heavyquark currents in the following way:

$$
\left(q_{\mu} q_{\nu}-g_{\mu \nu} q^{2}\right) \Pi\left(q^{2}\right)=i \int d^{4} x e^{i q \cdot x}\left\langle 0\left|T\left\{j_{\mu}^{\nu}(x) j_{\nu}^{\nu}(0)\right\}\right| 0\right\rangle,
$$

where $j_{\mu}^{v}(x) \equiv \bar{Q} \gamma_{\mu} Q(x)$. Using causality and the optical theorem one obtains

$$
P_{n}=\left.\frac{12 \pi^{2} e_{Q}^{2}}{n !}\left(\frac{d}{d q^{2}}\right)^{n} \Pi\left(q^{2}\right)\right|_{q^{2}=0}=\int_{\sqrt{s_{\min }}}^{\infty} \frac{d s}{s^{n+1}} R_{Q \bar{Q}}(s),
$$

where $R_{Q \bar{Q}} \equiv \sigma\left(e^{+} e^{-} \rightarrow Q \bar{Q}\right) / \sigma\left(e^{+} e^{-} \rightarrow \mu^{+} \mu^{-}\right)$and $e_{Q}$ is the quark electric charge. For low values of $n$, the lefthand side of Eq. (344) can be computed using perturbation theory due to the fact that the energy necessary to reach the threshold for heavy-quark production is much larger than $\Lambda_{\mathrm{QCD}},{ }^{26}$ whereas the right-hand side can be obtained from the experimental data. However, we are concerned here with the NR sum rules. These are defined by taking $n$ large. This implies the existence of new scales in the problem besides $m$ and $\Lambda_{\mathrm{QCD}}$, such as $m / \sqrt{n}, m / n$, and so on. Therefore it is not so clear that one can actually perform computations within perturbation theory. For $n$ large enough, one will have $\sqrt{n} \alpha_{s} \sim 1$ and a complete resummation of these terms should be achieved. The quantity $\sqrt{n} \alpha_{s}$ appears in the computation through the ratio of two different scales: $m \alpha_{s} /(m / \sqrt{n})$. Hence we see the following analogy with the NR situation: $1 / \sqrt{n}$ plays the same role as $v$, the velocity of the heavy quark, and by taking $\sqrt{n} \alpha_{s} \sim 1$ we are considering the NR limit.

There is also another problem. For sufficiently large $n$, we can no longer claim that the induced scales are much larger than $\Lambda_{\mathrm{QCD}}$ and nonperturbative effects need to be considered. How to handle them is a delicate issue. Here, we only consider $m / \sqrt{n} \gg \Lambda_{\mathrm{QCD}}$. This seems to be a safe requirement (at least for bottomonium). It is not clear, however, that we can also assume $m / n \gg \Lambda_{\mathrm{QCD}}$. In practical applications the boundary for doing so is usu-

\footnotetext{
${ }^{26}$ One should not forget, however, that potential problems may appear beyond NNLO due to the appearance of physical decay channels of the heavy quarkonium (Groote and Pivovarov, 2002; Portoles and Ruiz-Femenia, 2002).
} 
TABLE V. Recent determinations of $\bar{m}_{b}$ and $\bar{m}_{c}$ in the $\overline{\mathrm{MS}}$ scheme from NR sum rules.

\begin{tabular}{ccc}
\hline \hline Reference & Order & $\bar{m}_{b}\left(\bar{m}_{b}\right)(\mathrm{GeV})$ \\
\hline Melnikov and Yelkhovsky, 1999a & NNLO (kinetic mass) & $4.20 \pm 0.10$ \\
Penin and Pivovarov, 1999 & NNLO (pole mass) & $4.21 \pm 0.11$ \\
Beneke and Signer, 1999 & NNLO (PS mass) & $4.26 \pm 0.09$ \\
Hoang, 2000 & NNLO (1S mass) & $4.17 \pm 0.05$ \\
Eidemüller, 2003 & NNLO (PS mass) & $4.24 \pm 0.10$ \\
\hline Reference & Order & $\bar{m}_{c}\left(\bar{m}_{c}\right)(\mathrm{GeV})$ \\
\hline Eidemüller, 2003 & NNLO (PS mass) & $1.19 \pm 0.11$ \\
\hline \hline
\end{tabular}

ally taken around $n \sim 10$. We discuss this issue further below.

In spite of the above remarks, the NR sum rules are ideal from the experimental point of view. By taking $n$ large on the right-hand side of Eq. (344), the contribution from high momenta (the continuum region) is suppressed. Actually, this is the region which is less well known on the experimental side. Therefore by using NR sum rules, the experimental errors are significantly reduced. In practice, the following parametrization is used:

$$
P_{n}^{\mathrm{ex}}=\sum_{k=1}^{6} \frac{9 \pi}{\alpha^{2}(2 m)} \frac{\Gamma_{\Upsilon(k)}}{M_{\Upsilon(k)}^{(2 n+1)}}+\int_{\sqrt{s_{B B}}} \frac{d s}{s^{n+1}} r_{\text {cont }}(s) .
$$

The theoretical expressions for the moments $P_{n}^{\text {th }}$ can be computed order by order in the NR expansion in $1 / \sqrt{n}$ and $\alpha_{s}$, which at each order resums all the terms proportional to $\alpha_{s} \sqrt{n}$ to any power. Nowadays they are known in the on-shell scheme at NNLO in the NR expansion, which includes all corrections up to order $1 / n$, $\alpha_{s} / \sqrt{n}$, and $\alpha_{s}^{2}$ (Kuhn et al., 1998; Penin and Pivovarov, 1998; Beneke and Signer, 1999; Hoang, 1999; Melnikov and Yelkhovsky, 1999a). With this accuracy, the dispersion integration for the moments $P_{n}$ takes the form

$$
\begin{aligned}
P_{n}= & \frac{18 C_{A}}{4^{n} m^{2 n+2} \alpha^{2}(2 m)} \int_{E_{1}}^{\infty} \frac{d E}{m} \exp \left(-\frac{E}{m} n\right) \\
& \times\left(1-\frac{E}{2 m}+\frac{E^{2}}{4 m^{2}} n\right) \operatorname{Im}\left[\left\langle\mathbf{r}=0\left|G_{s}(E)\right| \mathbf{r}=0\right\rangle\right] \\
& \times\left(\operatorname{Im} f_{\mathrm{EM}}^{\mathrm{pNR}}\left({ }^{3} S_{1}\right)+\operatorname{Im} g_{\mathrm{EM}}^{\mathrm{pNR}}\left({ }^{3} S_{1}\right) \frac{E}{m}\right),
\end{aligned}
$$

where $E \equiv \sqrt{s}-2 m$ and $E_{1}$ is the binding energy of the lowest-lying resonance. The exponential form of the LO NR contribution to the energy integration has to be chosen because $E$ scales as $v^{2} \sim 1 / n$. For explicit expressions, we refer the reader to Hoang (1999).

As we have pointed out before, working in the onshell scheme introduces large errors. Therefore most of the analyses nowadays use threshold masses, where the cancellation of the pole-mass renormalon is explicit (see Table V). In practical terms this amounts to reexpressing the results obtained in the on-shell scheme in terms of the threshold masses. Nevertheless, even if some improvement is obtained, large uncertainties remain due to a rather strong scale dependence. This scale dependence can be traced back to the fact that the decay width of the heavy quarkonium to $e^{+} e^{-}$is strongly scale dependent. For a more detailed discussion of this point, see Beneke and Signer (1999). In this respect, RG techniques have not yet been applied to these computations. It would be most interesting to do that and to see whether a more stable result is obtained.

Nonperturbative effects in sum rules are parametrically of the same size as in the $\Upsilon(1 S)$ mass in the standard counting $1 / \sqrt{n} \sim \alpha_{s}$. Nevertheless, it may happen that they are numerically suppressed. This is indeed the case considering that one can describe the nonperturbative effects by local condensates (Voloshin, 1995; Onishchenko, 2000). However, one can use the expression in terms of local condensates only when $m / n \gg \Lambda_{\mathrm{QCD}}$ (although one can use that result as an order of magnitude estimate of the nonperturbative effects). This would be analogous to the assumption $m \alpha_{s}^{2} \gg \Lambda_{\mathrm{QCD}}$, which may be difficult to fulfill. Therefore it is more likely that the nonperturbative corrections will also depend on a nonlocal condensate of the same type (chromoelectric correlator) as the $\Upsilon(1 S)$ mass does. Thus in order to estimate the nonperturbative errors in sum-rules evaluations, it would be most welcome to have at least the explicit expression of the nonperturbative effects when $m / n \sim \Lambda_{\mathrm{QCD}}$, which is still lacking. In that way one could relate the nonperturbative effects for different moments in the sum rules to each other or to the nonperturbative effects in the $Y(1 S)$ mass.

\section{F. $t-\bar{t}$ production near threshold}

Future linear electron-positron colliders will produce large samples of $t-\bar{t}$ pairs near threshold (Bagger et al., 2000; Abe et al., 2001a, 2001b; Aguilar-Saavedra et al., 2001). In this regime, the top and the antitop will move slowly with respect to each other and pNRQCD becomes applicable. Since the top-quark mass $m_{t}$ $\sim 175 \mathrm{GeV}$ and the expected (electroweak) decay width $\Gamma_{t} \sim 1.5 \mathrm{GeV}$ are large in comparison with $\Lambda_{\mathrm{QCD}}$, nonperturbative effects due to $\Lambda_{\mathrm{QCD}}$ are expected to be small in the whole threshold region and hence a weak- 
coupling analysis is very reliable. In addition, since $\Gamma_{t}$ $\sim m_{t} \alpha_{s}^{2}$, which is the US scale, a remnant of the would-be toponium $1 S$ state is expected to show up as a bump in the total cross section. This will serve to obtain the top-quark mass with a high accuracy.

The $t-\bar{t}$ pair will be dominantly produced via $e^{+} e^{-}$ $\rightarrow \gamma^{*}, Z^{*} \rightarrow t \bar{t}$. The total production cross section may be written as (Hoang et al., 2000)

$$
\sigma_{\text {tot }}^{\gamma, Z}(s)=\frac{4 \pi \alpha^{2}}{3 s}\left[F^{v}(s) R^{v}(s)+F^{a}(s) R^{a}(s)\right],
$$

where $F^{v}(s)$ and $F^{a}(s)$ contain electroweak parameters (Hoang et al., 2002) and

$$
\begin{aligned}
& R^{v}(s)=\frac{4 \pi}{s} \operatorname{Im}\left(-i \int d^{4} x e^{i q \cdot x}\left\langle 0\left|T j_{\mu}^{v}(x) j^{\nu \mu}(0)\right| 0\right\rangle\right), \\
& R^{a}(s)=\frac{4 \pi}{s} \operatorname{Im}\left(-i \int d^{4} x e^{i q \cdot x}\left\langle 0\left|T j_{\mu}^{a}(x) j^{a \mu}(0)\right| 0\right\rangle\right),
\end{aligned}
$$

where $q=(\sqrt{s}, 0)$ and $j_{\mu}^{v}\left(j_{\mu}^{a}\right)$ is the vector (axial-vector) current that produces a quark-antiquark pair defined by Eq. (12) [Eq. (13)]. Hence the full QCD calculation can be split into (i) calculating the matching coefficients $b_{1}^{v}\left(m_{t}, \nu\right), b_{2}^{v}\left(m_{t}, \nu\right)$, and $b_{1}^{a}\left(m_{t}, \nu\right)$, and (ii) calculating current correlators in pNRQCD. Up to NNLO $\left[\mathcal{O}\left(\alpha_{s}^{2}\right)\right.$ corrections], the latter reduces to a purely quantummechanical calculation along the lines of Sec. IV.G (US gluons do not play any role). The potential is only needed at the order displayed in Eqs. (101) and (102). This calculation has been carried out by several groups ${ }^{27}$ and the final outcome is summarized by Hoang et al. (2000) [previous computations at LO (Fadin and Khoze, 1988) and NLO (Strassler and Peskin, 1991) relied on potential models which needed phenomenological input]. Several comments are in order.

(1) At NNLO, the scale dependence which appears in the matching coefficient $b_{1}^{v}\left(m_{t}, \nu\right)\left(b_{2}^{v}=b_{1}^{a}=1\right.$ at this order) is compensated by the scale dependence introduced by regulating and renormalizing the UV divergences of the quantum-mechanical perturbation theory (potential loops).

(2) The top-quark width is introduced by replacing $m_{t}$ by $m_{t}-i \Gamma_{t} / 2$. A consistent inclusion of electroweak effects is still lacking.

(3) In order to obtain stable results for the top-quark mass in going from LO to NLO to NNLO, it is very important to use the so-called threshold masses rather than the pole mass. These are discussed in Sec. V.

(4) The large logarithms arising due to the various scales in the problem can be resummed using RG techniques as described in Sec. IV.H. This problem is nontrivial because all scales (hard, soft, potential, and US) play a role. It was first addressed within the velocity

\footnotetext{
${ }^{27}$ For an analytical expression for the $\gamma \gamma \rightarrow t \bar{t}$ cross section at NNLO, see Penin and Pivovarov (2001).
}

NRQCD framework (Hoang, Manohar, Stewart, et al., 2001). However, the correct result for $b_{1}^{v}\left(m_{t}, \mu\right)$ at NLLO was first given within pNRQCD by Pineda (2002a) and later reproduced within velocity NRQCD (Hoang and Stewart, 2003). Hoang and co-workers (2004); Hoang et al. (2002) computed some partial results for the NNLLO contribution. The resulting series (Hoang, 2004) does not show a very good convergence (even if the absolute value of the corrections is small). This, however, may be due to the scheme dependence of the result. Penin et al. (2004b) have obtained a complete (and therefore scheme-independent) result with NNLLO accuracy for the ratio of the spin- 1 and spin- 0 production. In this case good convergence is found, but one should keep in mind that this ratio is less sensitive to the US scale than the full current. Therefore it is premature to draw any definite conclusion about the convergence of the series before getting the complete NNLLO evaluation, which, even if difficult, is within reach. This is of utmost importance for future determinations of the top mass and the Higgs-top coupling at a future linear collider (Martinez and Miquel, 2003).

(5) At NNNLO, as well as for the resummations above, US gluons start to play a role. The double logarithmic contributions were calculated by Kniehl and Penin (2000c) and the single logarithmic ones by Kniehl et al. (2003). The finite pieces are still missing. These can, in principle, be calculated with the potentials given by Kniehl, Penin, Smirnov, et al. (2002) together with the three-loop static potential (which is still missing), and the LO terms for the US gluons given by Eq. (60). The matching coefficients $b_{1}^{v}\left(m_{t}, \nu\right), b_{2}^{v}\left(m_{t}, \nu\right)$, and $b_{1}^{a}\left(m_{t}, \nu\right)$ also need to be calculated to one order higher in $\alpha_{s}$.

Figure 22 shows the current status of theoretical results for the total cross section for $e^{+} e^{-} \rightarrow \gamma^{*} \rightarrow t^{+} t^{-}$.

\section{G. Semi-inclusive radiative decays}

We have seen that NRQCD and pNRQCD are particularly suitable for describing inclusive decays of heavy quarkonia to light particles. Semi-inclusive and fully exclusive decays can also be addressed but they require additional theoretical considerations. Similar to what happens for inclusive decays, pNRQCD is expected to provide supplementary information here as well. Semiinclusive radiative decays to light hadrons in which only the photon energy is measured are the simplest of them and will be briefly discussed in the following.

We shall restrict our discussion to the so-called direct contributions, for which the photon is emitted from a heavy-quark electromagnetic current. Fragmentation contributions also play an important role (Catani and Hautmann, 1995). The starting point is the QCD formula (Rothstein and Wise, 1997)

$$
\frac{d \Gamma}{d z}=z \frac{M}{16 \pi^{2}} \operatorname{Im} T(z),
$$



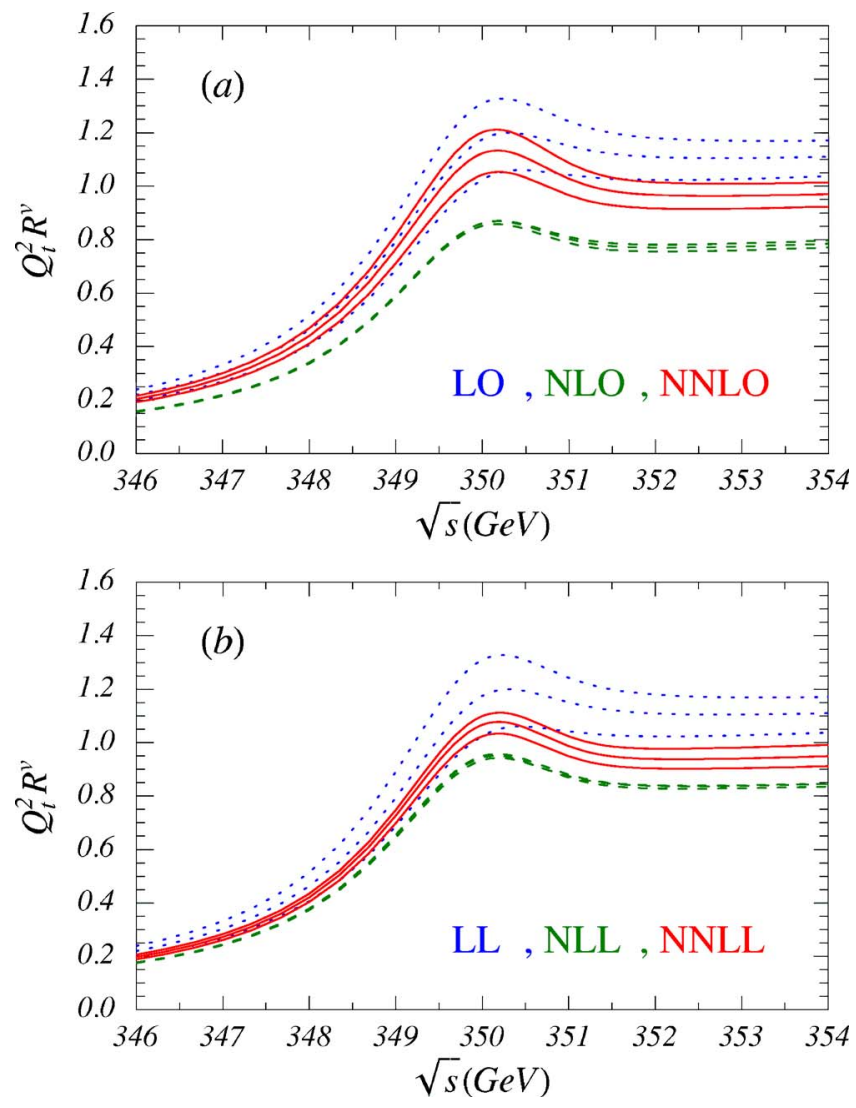

FIG. 22. (Color online) (a) The results for $e_{t}^{2} R^{v}\left(e_{t}=2 / 3\right.$ is the top-quark electric charge) with $m_{t}=175 \mathrm{GeV}$ (the $1 S$ threshold mass is used, see Sec. V) and $\Gamma_{t}=1.43 \mathrm{GeV}$ in fixed-order perturbation theory at LO (dotted lines), NLO (dashed lines), and NNLO (solid lines). (b) The results for $e_{t}^{2} R^{v}$ with the same parameters in RG-improved perturbation theory at LL (dotted lines), NLL (dashed lines), and (partial) NNLL (solid lines) order. For each order, curves are plotted for $\nu_{p} / m_{t}=0.15,0.20$, and 0.3. From Hoang, 2003.

$$
\begin{aligned}
T(z)= & -i \int d^{4} x e^{-i q \cdot x}\left\langle V_{Q}(n S)\right| T\left\{j_{\mu}^{\nu}(x) j_{\nu}^{\nu}(0)\right\} \\
& \times\left|V_{Q}(n S)\right\rangle g_{\perp}^{\mu \nu}
\end{aligned}
$$

where $M$ is the heavy-quarkonium mass, and we have restricted ourselves to ${ }^{3} S_{1}$ states. $q$ is the photon momentum, which in the rest frame of the heavy quarkonium is $q=\left(q_{+}, q_{-}, q_{\perp}\right)=(z M, 0,0)$. We have used lightcone coordinates $q_{ \pm}=q^{0} \pm q^{3} . z \in[0,1]$ is defined as $z$ $=2 E_{\gamma} / M$, namely, the fraction of the maximum energy that the photon may have in the heavy-quarkonium rest frame.

For $z$ away from the lower and upper end points (0 and 1, respectively), no further scale is introduced beyond those inherent in the NR system. The integration of the scale $m$ in the time-ordered product of currents in Eq. (349) leads to local NRQCD operators with matching coefficients which depend on $m$ and $z$. At LO one obtains

$$
\begin{aligned}
\frac{1}{\Gamma_{0}} \frac{d \Gamma_{\mathrm{LO}}}{d z}= & \frac{2-z}{z}+\frac{z(1-z)}{(2-z)^{2}}+2 \frac{1-z}{z^{2}} \ln (1-z) \\
& -2 \frac{(1-z)^{2}}{(2-z)^{3}} \ln (1-z),
\end{aligned}
$$

where

$$
\Gamma_{0}=\frac{32}{27} \alpha \alpha_{s}^{2} e_{Q}^{2} \frac{\left\langle V_{Q}(n S)\left|\mathcal{O}_{1}\left({ }^{3} S_{1}\right)\right| V_{Q}(n S)\right\rangle}{m^{2}},
$$

and $e_{Q}$ is the charge of the heavy quark. The $\alpha_{s}$ correction to this rate was calculated numerically by Krämer (1999). The contribution of color-octet operators turns out to be strongly suppressed away from the upper endpoint region (the lowest-order color-octet contribution identically vanishes) (Maltoni and Petrelli, 1999). The expression corresponding to Eq. (351) in pNRQCD is obtained at lowest order in any of the possible regimes by just making the substitution

$$
\left\langle V_{Q}(n S)\left|\mathcal{O}_{1}\left({ }^{3} S_{1}\right)\right| V_{Q}(n S)\right\rangle=\frac{N_{c}}{2 \pi}\left|R_{n 0}(0)\right|^{2} .
$$

The final result coincides with the result of early QCD calculations (Brodsky et al., 1978; Koller and Walsh, 1978).

For $z \rightarrow 0$, the emitted low-energy photon can only produce transitions within the NR bound state without destroying it. Hence the direct low-energy photon emission takes place in two steps: (i) the photon is emitted (dominantly by electric dipole and magnetic dipole transitions) and (ii) the remaining (off-shell) bound state is annihilated into light hadrons. It has a suppression $\sim z^{3}$ with respect to $\Gamma_{0}$ [see Manohar and Ruiz-Femenia (2004) and Voloshin (2004) for recent analyses of this region in QED]. Hence at some point the direct photon emission is overtaken by the so-called fragmentation contributions $\bar{Q} Q \rightarrow g g g \rightarrow g g \bar{q} q \gamma$ (Catani and Hautmann, 1995; Maltoni and Petrelli, 1999).

For $z \rightarrow 1$, momentum conservation implies that the gluons emitted in the short-distance annihilation process must have a direction roughly opposite to that of the photon. They produce a jetlike event with momentum $p_{X}=((1-z) M, M, 0)$ (in light-cone coordinates). This implies that two more scales become relevant, $p_{X_{+}}=(1$ $-z) M$ and $p_{X}^{2}=(1-z) M^{2}$, producing an additional hierarchy $M \gg M \sqrt{1-z} \gg M(1-z)$. In recent years an EFT named soft-collinear effective theory (SEFT) has been introduced in order to efficiently exploit this hierarchy of scales. The main ideas which led to SEFT were outlined by Bauer, Fleming, and Luke (2001). Nowadays, it is being developed by several groups (Bauer, Fleming, Pirjol, et al., 2001; Beneke et al., 2002; Chay and Kim, 2002; Hill and Neubert, 2003) and it has been applied to Y(1S) radiative decays by Bauer, Chaing, et al. (2001); Fleming and Leibovich (2003a, 2003b, 2004); Garcia i Tormo and Soto (2004). We shall not review SEFT here (a complete analysis connecting pNRQCD and SEFT is still lacking), but only mention its relevant features for the case we are concerned with. For $z \rightarrow 1$, upon inte- 
grating out the scale $m$, the time-ordered product of currents in Eq. (349) does not reduce to local NRQCD operators anymore. Additional degrees of freedom are needed. These are collinear gluons (and collinear light quarks), which are defined as those having a typical momentum (in light-cone coordinates) $\quad p \sim((1$ $-z) M, M, \sqrt{1-z} M)$. They are incorporated in SEFT together with the remaining degrees of freedom in NRQCD. Then one matches the QCD electromagnetic currents $j_{\mu}^{v}(x)$ (rather than the full time-ordered product) to SEFT currents. Next, the scale $\sqrt{1-z} M$ is integrated out (assuming that it is large enough to use perturbation theory) by matching the time-ordered product of currents in SEFT (now renamed $\mathrm{SCET}_{\mathrm{I}}$ ) to (nonlocal) operators of the so-called $\mathrm{SCET}_{\mathrm{II}}$, which does not contain collinear modes of virtuality $\sim(1-z) M^{2}$ anymore [see Beneke and Feldmann (2004) for a detailed description of the modes involved in $\mathrm{SCET}_{\mathrm{I}}$ and in $\mathrm{SCET}_{\mathrm{II}}$. By calculating the anomalous dimensions of the various operators appearing in both matchings and using standard RG techniques, one can resum large (Sudakov) logarithms $\ln (1-z)$. For the color-octet currents, this was done by Bauer, Chiang, et al. (2001) and for the color-singlet ones by results of Fleming and Leibovich (2003a, 2004). For the color-octet sector, the final outcome corrects the old results obtained by Photiadis (1985). For the color-octet sector, the final result may be given in terms of so-called shape functions (Rothstein and Wise, 1997), which involve expectation values in the heavy-quarkonium state of two color-octet NRQCD currents separated along a light-cone direction, for instance,

$$
\begin{aligned}
S\left(\ell^{+}\right)= & \int \frac{d x^{-}}{4 \pi} e^{-i / 2 \ell^{+} x^{-}}\left\langle V_{Q}(n S)\right|\left[\psi^{\dagger} T^{b} \chi\right]\left(x^{-}\right) \phi_{b c}^{\mathrm{adj}}\left(0, x^{-}\right) \\
& \times\left[\chi^{\dagger} T^{c} \psi\right](0)\left|V_{Q}(n S)\right\rangle .
\end{aligned}
$$

If the heavy-quarkonium state is in the weak-coupling regime, as is likely in the case of the $Y(1 S)$ system, one can use pNRQCD in that regime to calculate the shape functions. This was done by Garcia i Tormo and Soto (2004) [see also Beneke et al. (2000)]. When these results are combined with those of the singlet sector, an excellent description of data (Nemati et al., 1997) is obtained (see Fig. 23) for the end-point region. Although, as discussed by Garcia i Tormo and Soto (2004), there are still some calculations missing in order to have a totally unambiguous theoretical result, the agreement with data is very encouraging. Indeed, the end-point region of the photon spectrum has been very elusive to theoretical descriptions. The color-singlet contribution (sometimes referred to as the color-singlet model) lies well above the data. For the color-octet contributions, different models were used in the past to estimate the shape functions, generically producing results incompatible with data (Bauer, Chiang, et al., 2001; Wolf, 2001). These facts were used to argue that the introduction of a nonvanishing gluon mass was necessary in order to fit the experimental data (Field, 2002). This is no longer the case, at least as far as the $\mathrm{Y}(1 S)$ system is concerned.

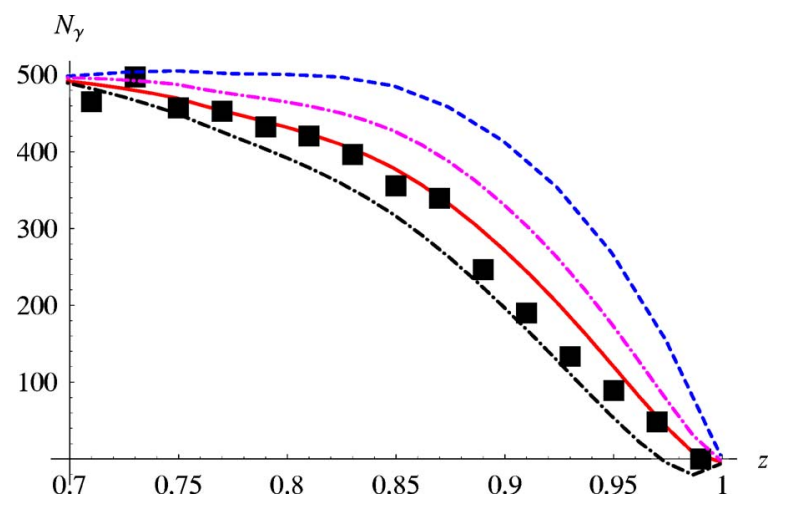

FIG. 23. (Color online) End-point region of the photon spectrum in semi-inclusive $Y$ decay. The points are CLEO data (Nemati et al., 1997), the dashed line is the (best) curve obtained by Fleming and Leibovich (2003a), and the solid and dot-dashed lines are the results of Garcia i Tormo and Soto (2004) (the solid line is the central value and the dot-dashed lines are obtained by a $2^{ \pm 1}$ variation of the relevant scale). From Garcia i Tormo and Soto, 2004.

\section{CONCLUSIONS}

The application of QCD EFTs to heavy quarkonia has considerably increased our understanding of those systems from a fundamental point of view. This has occurred at several levels.

- Long-standing puzzles have been resolved. For instance, the fate of IR divergences in the decay widths to light particles has been resolved in NRQCD by introducing color-octet operators, and the fate of the IR divergences in the QCD static potential has been resolved in PNRQCD by the explicit use of US gluons.

- Heavy-quarkonium physics in the strong-coupling regime has been brought into the realm of systematic calculations in QCD. This has led to the discovery of new terms in the potential which were missed in the past, both analytic and nonanalytic in $1 / \mathrm{m}$, and to express the color-octet NRQCD matrix elements in terms of wave functions at the origin and additional bound-state-independent nonperturbative parameters. This puts NR phenomenological potential models in a QCD context in the kinematic regime where this EFT description applies.

- In the weak-coupling regime, it has allowed higherorder calculations to be carried out in a systematic and much simpler manner. Errors are under parametric control. Moreover, it has made possible the application of RG techniques, which have been used to resum infinite series of IR QCD logarithms, being so far the only known way to carry out such resummations. This has opened up the possibility of having precision determinations of the Standard Model parameters to which the heavy quarkonium is sensitive: $\alpha_{s}$ and the heavy-quark masses.

Although the virtues by far exceed the drawbacks, the 
latter are not absent in EFTs of heavy quarkonium. They are all related to the fact that the actual bottom and especially charm masses, are not so much larger than $\Lambda_{\mathrm{OCD}}$ (for toponiumlike states the EFT should work very well). This means that the scales $m \gg p \gg E$, which are assumed to be well separated, may actually not be that well separated, and hence expansions in various ratios may show a slow convergence. In the strongcoupling regime it is still too early to judge. For $Y(1 S)$ in the weak-coupling regime, the convergence seems to be good. In addition, most of the matching coefficients of NRQCD show a poor convergence in $\alpha_{s}(m)$ for both bottom and charm masses, which is jeopardizing many practical applications of NRQCD. We may expect, however, that once the renormalon singularities in each series have been identified and properly subtracted, the situation will improve considerably, as has occurred with the introduction of threshold masses.

\section{PROSPECTS}

NR EFTs for heavy quarkonium still have an enormous potential and may evolve in many different directions. Some of them are more or less obvious improvements or extensions of what has been presented here. Others require the introduction of new concepts and techniques.

Among the obvious improvements are those which consist of calculating matching coefficients and observables to a higher accuracy in both NRQCD and pNRQCD. In NRQCD, it would be important to have the NNLO calculation of the imaginary parts of the matching coefficients of the four-fermion operators, at least for $S$ and $P$ waves. This would allow one to see whether the poor convergence observed at NLO is corrected or remains, and in either case it would facilitate renormalon-based improvements. It would also be important to have further and more accurate lattice calculations of the NRQCD matrix elements (see Sec. II.F.2). In the weak-coupling regime of $\mathrm{pNRQCD}$, some perturbative calculations are missing, which seem to be in reach of the current computational power. Let us only mention the complete three-loop static potential, which is necessary for the complete NNNLO spectrum and for electromagnetic production processes (for instance, in $t-\bar{t}$; ; the complete NNLLO resummation of the creation and annihilation currents; and the NNNLO calculation of electromagnetic production. These would allow an increase in the precision of the determinations of $m_{b}, m_{c}$, $\alpha_{s}$, and, eventually, $m_{t}$ (see Sec. VIII.A). For the case of $\Upsilon(1 S)$, the accuracy is limited by the poor knowledge of the nonperturbative contributions, which are precisely given in terms of chromoelectric-field correlators. A proper lattice evaluation of the latter would be most welcome. For the $t-\bar{t}$ system, the level of accuracy calls for the consistent inclusion of electroweak effects, which is also missing (see Sec. VIII.F).

In the strong-coupling regime of $\mathrm{pNRQCD}$, on the one hand, it is necessary to update the early lattice evaluations of the potentials including the more recently found $1 / \mathrm{m}$ and $1 / \mathrm{m}^{2}$ potentials. On the other hand, a systematic matching procedure of the potentials to the continuum limit and a rigorous lattice renormalization scheme should be developed (see Sec. VII.G). This will lead to a fully consistent lattice version of pNRQCD.

In the same regime, the inclusion of pseudoGoldstone bosons (pions) and low-energy photons is still lacking. This would allow a description of electromagnetic and hadronic transitions in that situation.

Let us next mention some applications of EFTs to heavy quarkonium that require further theoretical elaborations.

The systematic study of semi-inclusive (see Sec. VIII.G) and exclusive decays may need the introduction of further degrees of freedom in addition to those of NRQCD or pNRQCD.

NRQCD production matrix elements should also have definite expressions in pNRQCD both in the weakand in the strong-coupling regimes, which have not been worked out yet. It is expected that as for the decay matrix elements, new relations may appear and the number of nonperturbative parameters consequently reduced.

States close to or above the heavy-light meson pair threshold cannot be treated using pNRQCD, at least in its current formulations. Hence one has to stay at the NRQCD level. A hadronic version of NRQCD, including heavy-quarkonium states, heavy-light mesons, and pseudo-Goldstone bosons, in the spirit of Burdman and Donoghue (1992); Casalbuoni et al. (1997); Mannel and Urech (1997); Voloshin (2003), might prove useful and will eventually help to understand the nature of present (Choi et al., 2003) and possibly future potential states in that region.

Including finite temperature in NRQCD and pNRQCD would make it possible to address important questions such as $J / \psi$ suppression as a sign of deconfinement (Matsui and Satz, 1986) in current and future heavy-ion collision experiments.

Finally, by slightly changing the fundamental degrees of freedom, EFTs may be built which are similar to pNRQCD, but also suitable for describing bound states made of two heavy particles other than heavy quarkonium. An example is heavy baryons made of two heavy quarks, similar to those recently discovered at SELEX (Mattson et al., 2002). These systems are theoretically quite interesting due to the interplay of HQET and NRQCD (Rösch, 2003; Soto, 2003). Quarkoniaquarkonia scattering may also be studied along the lines of the work of Bhanot and Peskin (1979); Peskin (1979); Fujii and Kharzeev (1999); Vairo (2000).

We feel that we are at the beginning of a time where most aspects of the physics of heavy quarkonium, and of similar systems, will be addressed in terms of EFTs of QCD. This is more than a change in language. It is moving this physics from being a battleground of competing models to being a source of some of the fundamental parameters of the Standard Model, a reliable test of its validity in the strong interaction sector, and a unique laboratory for the study of QCD properties. 


\section{ACKNOWLEDGMENTS}

We are thankful to A. G. Grozin for comments on the manuscript and to C. Ewerz for a careful reading of the manuscript and many useful comments and suggestions. We gratefully acknowledge financial support from "Azioni Integrate Italia-Spagna 2004 (IT1824)/Acciones Integradas España-Italia (HI2003-0362)," and from the cooperation agreement INFN04-16 (CICYT-INFN). A.P. and J.S. are also funded by the MCyT and Feder (Spain) Grant No. FPA2001-3598, the CIRIT (Catalonia) Grant No. 2001SGR-00065, and the network EURIDICE (EU) HPRN-CT2002-00311. J.S. has been partially funded by the National Science Foundation under Grant No. PHY99-0794. A.V. aknowledges the financial support obtained inside the Italian MIUR program "Incentivazione alla Mobilità di Studiosi Stranieri e Italiani Residenti all'Estero." N.B., J.S., and A.V. would like to thank the Benasque Center of Physics and J.S. the Kvali Institute of Theoretical Physics for hospitality while part of this work was written.

\section{TABLE OF ACRONYMS}

$\begin{array}{ll}\text { 2PI } & \text { Two-particle irreducible } \\ \text { 2PR } & \text { Two-particle reducible } \\ \text { DR } & \text { Dimensional regularization } \\ \text { EFT } & \text { Effective field theory } \\ \text { IR } & \text { Infrared } \\ \text { HQET } & \text { Heavy-quark effective theory } \\ \text { LO } & \text { Leading order } \\ \text { MS } & \text { Minimal subtraction } \\ \text { NLO } & \text { Next-to-leading order } \\ \text { NNLO } & \text { Next-to-next-to-leading order } \\ \text { NNNLO } & \text { Next-to-next-to-next-to-leading order } \\ \text { LL } & \text { Leading-logarithm order } \\ \text { NLL } & \text { Next-to-leading-logarithm order } \\ \text { NNLL } & \text { Next-to-next-to-leading-logarithm order } \\ \text { NR } & \text { Nonrelativistic } \\ \text { NRQCD } & \text { Nonrelativistic quantum chromodynamics } \\ \text { NRQED } & \text { Nonrelativistic quantum electrodynamics } \\ \text { pNRQCD Potential NRQCD } \\ \text { PS } & \text { Potential subtracted } \\ \text { QFT } & \text { Quantum field theory } \\ \text { RG } & \text { Renormalization group } \\ \text { RS } & \text { Renormalon subtracted } \\ \text { SCET } & \text { Soft-collinear effective theory } \\ \text { US } & \text { Ultrasoft } \\ \text { vNRQCD } & \text { Velocity NRQCD }\end{array}$

\section{REFERENCES}

Abe, F., et al. (CDF Collaboration), 1994, Phys. Rev. Lett. 73, 225.

Abe, F., et al. (CDF Collaboration), 1998, Phys. Rev. Lett. 81, 2432.

Abe, K., et al. (ACFA Linear Collider Working Group), 2001a, e-print hep-ph/0109166.

Abe, T., et al. (American Linear Collider Working Group), 2001b, e-print hep-ex/0106057.
Aglietti, U., and Z. Ligeti, 1995, Phys. Lett. B 364, 75.

Aguilar-Saavedra, J. A., et al. (ECFA/DESY LC Physics Working Group), 2001, e-print hep-ph/0106315.

Amoros, G., M. Beneke, and M. Neubert, 1997, Phys. Lett. B 401, 81 .

Appelquist, T., M. Dine, and I. J. Muzinich, 1978, Phys. Rev. D 17, 2074.

Appelquist, T., and H. D. Politzer, 1975, Phys. Rev. Lett. 34, 43.

Aubert, J. J., et al., 1974, Phys. Rev. Lett. 33, 1404.

Augustin, J. E., et al., 1974, Phys. Rev. Lett. 33, 1406.

Bagger, J., et al. (American Linear Collider Working Group), 2000, e-print hep-ex/0007022.

Baker, M., J. S. Ball, N. Brambilla, G. M. Prosperi, and F. Zachariasen, 1996, Phys. Rev. D 54, 2829.

Baker, M., J. S. Ball, N. Brambilla, and A. Vairo, 1996, Phys. Lett. B 389, 577.

Baker, M., J. S. Ball, and F. Zachariasen, 1991, Phys. Rep. 209, 73.

Baker, M., N. Brambilla, H. G. Dosch, and A. Vairo, 1998, Phys. Rev. D 58, 034010.

Baker, M., and R. Steinke, 2001, e-print hep-ph/0101255.

Bali, G. S., 2001, Phys. Rep. 343, 1.

Bali, G. S., N. Brambilla, and A. Vairo, 1998, Phys. Lett. B 421, 265.

Bali, G. S., and A. Pineda, 2004, Phys. Rev. D 69, 094001.

Bali, G. S., K. Schilling, and A. Wachter, 1997, Phys. Rev. D 56, 2566.

Bali, G. S., et al. (UKQCD Collaboration), 1993, Phys. Lett. B 309, 378.

Bali, G. S., et al. (TXL Collaboration), 2000, Phys. Rev. D 62, 054503.

Balitsky, I. I., 1985, Nucl. Phys. B 254, 166.

Balzereit, C., 1999, Phys. Rev. D 59, 034006.

Barbieri, R., M. Caffo, R. Gatto, and E. Remiddi, 1980, Phys. Lett. B95, 93.

Barbieri, R., M. Caffo, R. Gatto, and E. Remiddi, 1981, Nucl. Phys. B 192, 61.

Barbieri, R., E. d'Emilio, G. Curci, and E. Remiddi, 1979, Nucl. Phys. B 154, 535.

Barbieri, R., R. Gatto, and E. Remiddi, 1976, Phys. Lett. 61B, 465.

Barchielli, A., N. Brambilla, and G. M. Prosperi, 1990, Nuovo Cimento Soc. Ital. Fis., A 103A, 59.

Barchielli, A., E. Montaldi, and G. M. Prosperi, 1988, Nucl. Phys. B 296, 625.

Bauer, C. W., C.-W. Chiang, S. Fleming, A. K. Leibovich, and I. Low, 2001, Phys. Rev. D 64, 114014.

Bauer, C. W., S. Fleming, and M. E. Luke, 2001, Phys. Rev. D 63, 014006.

Bauer, C. W., S. Fleming, D. Pirjol, and I. W. Stewart, 2001, Phys. Rev. D 63, 114020.

Bauer, C. W., and A. V. Manohar, 1998, Phys. Rev. D 57, 337. Becher, T., and K. Melnikov, 2002, Phys. Rev. D 66, 074508. Becher, T., and K. Melnikov, 2003, Phys. Rev. D 68, 014506.

Beneke, M., 1995, Phys. Lett. B 344, 341.

Beneke, M., 1997, e-print hep-ph/9703429.

Beneke, M., 1998, Phys. Lett. B 434, 115.

Beneke, M., 1999, Phys. Rep. 317, 1.

Beneke, M., and V. M. Braun, 1994, Nucl. Phys. B 426, 301.

Beneke, M., A. P. Chapovsky, M. Diehl, and T. Feldmann, 2002, Nucl. Phys. B 643, 431.

Beneke, M., and T. Feldmann, 2004, Nucl. Phys. B 685, 249. 
Beneke, M., G. A. Schuler, and S. Wolf, 2000, Phys. Rev. D 62, 034004.

Beneke, M., and A. Signer, 1999, Phys. Lett. B 471, 233.

Beneke, M., A. Signer, and V. A. Smirnov, 1998, Phys. Rev. Lett. 80, 2535.

Beneke, M., A. Signer, and V. A. Smirnov, 1999, Phys. Lett. B 454, 137.

Beneke, M., and V. A. Smirnov, 1998, Nucl. Phys. B 522, 321.

Bethe, H. A., and E. Salpeter, 1951, Phys. Rev. 84, 1232.

Bhanot, G., and M. E. Peskin, 1979, Nucl. Phys. B 156, 391.

Bigi, I. I. Y., M. A. Shifman, N. G. Uraltsev, and A. I. Vainshtein, 1994, Phys. Rev. D 50, 2234.

Billoire, A., 1980, Phys. Lett. B92, 343.

Blok, B., J. G. Körner, D. Pirjol, and J. C. Rojas, 1997, Nucl. Phys. B 496, 358.

Bodwin, G. T., E. Braaten, and G. P. Lepage, 1992, Phys. Rev. D 46, 1914.

Bodwin, G. T., E. Braaten, and G. P. Lepage, 1995, Phys. Rev. D 51, 1125.

Bodwin, G. T., and Y.-Q. Chen, 1999, Phys. Rev. D 60, 054008. Bodwin, G. T., and Y.-Q. Chen, 2001, Phys. Rev. D 64, 114008.

Bodwin, G. T., J. Lee, and R. Vogt, 2003, e-print hep-ph/ 0305034.

Bodwin, G. T., and A. Petrelli, 2002, Phys. Rev. D 66, 094011. Bodwin, G. T., D. K. Sinclair, and S. Kim, 1996, Phys. Rev. Lett. 77, 2376.

Bodwin, G. T., D. K. Sinclair, and S. Kim, 2002, Phys. Rev. D 65, 054504.

Bolder, B., et al., 2001, Phys. Rev. D 63, 074504.

Born, K. D., E. Laermann, T. F. Walsh, and P. M. Zerwas, 1994, Phys. Lett. B 329, 332.

Braaten, E., and Y.-Q. Chen, 1998, Phys. Rev. D 57, 4236.

Braaten, E., S. Fleming, and T. C. Yuan, 1996, Annu. Rev. Nucl. Part. Sci. 46, 197.

Brambilla, N., 1998, e-print hep-ph/9809263.

Brambilla, N., 2000, e-print hep-ph/0012211.

Brambilla, N., P. Consoli, and G. M. Prosperi, 1994, Phys. Rev. D 50, 5878.

Brambilla, N., D. Eiras, A. Pineda, J. Soto, and A. Vairo, 2002, Phys. Rev. Lett. 88, 012003.

Brambilla, N., D. Eiras, A. Pineda, J. Soto, and A. Vairo, 2003, Phys. Rev. D 67, 034018.

Brambilla, N., D. Gromes, and A. Vairo, 2001, Phys. Rev. D 64, 076010.

Brambilla, N., D. Gromes, and A. Vairo, 2003, Phys. Lett. B 576, 314.

Brambilla, N., A. Pineda, J. Soto, and A. Vairo, 1999a, Phys. Lett. B 470, 215.

Brambilla, N., A. Pineda, J. Soto, and A. Vairo, 1999b, Phys. Rev. D 60, 091502.

Brambilla, N., A. Pineda, J. Soto, and A. Vairo, 2000, Nucl. Phys. B 566, 275.

Brambilla, N., A. Pineda, J. Soto, and A. Vairo, 2001, Phys. Rev. D 63, 014023.

Brambilla, N., A. Pineda, J. Soto, and A. Vairo, 2004, Phys. Lett. B 580, 60.

Brambilla, N., Y. Sumino, and A. Vairo, 2001, Phys. Lett. B 513, 381.

Brambilla, N., Y. Sumino, and A. Vairo, 2002, Phys. Rev. D 65, 034001.

Brambilla, N., and A. Vairo, 1997, Phys. Rev. D 55, 3974.

Brambilla, N., and A. Vairo, 1999a, e-print hep-ph/9904330.

Brambilla, N., and A. Vairo, 1999b, Nucl. Phys. B, Proc. Suppl.
74, 201.

Brambilla, N., and A. Vairo, 2000a, Phys. Rev. D 62, 094019.

Brambilla, N., and A. Vairo, 2000b, e-print hep-ph/0004192.

Brambilla, N., et al., 2004, e-print hep-ph/0412158.

Brodsky, S. J., D. G. Coyne, T. A. DeGrand, and R. R. Horgan, 1978, Phys. Lett. 73B, 203.

Brown, L. S., and W. I. Weisberger, 1979, Phys. Rev. D 20, 3239.

Buchmüller, W., Y. J. Ng, and S. H. H. Tye, 1981, Phys. Rev. D 24, 3003.

Burdman, G., and J. F. Donoghue, 1992, Phys. Lett. B 280, 287. Campostrini, M., 1985, Nucl. Phys. B 256, 717.

Campostrini, M., A. Di Giacomo, and S. Olejnik, 1986, Z. Phys. C 31, 577.

Capitani, S., M. Luscher, R. Sommer, and H. Wittig (ALPHA Collaboration), 1999, Nucl. Phys. B 544, 669.

Casalbuoni, R., et al., 1997, Phys. Rep. 281, 145.

Caswell, W. E., and G. P. Lepage, 1986, Phys. Lett. 167B, 437.

Catani, S., and F. Hautmann, 1995, Nucl. Phys. B, Proc. Suppl. 39BC, 359.

Chay, J., and C. Kim, 2002, Phys. Rev. D 65, 114016.

Chen, Y.-Q., Y.-P. Kuang, and R. J. Oakes, 1995, Phys. Rev. D 52, 264.

Chetyrkin, K. G., and M. Steinhauser, 2000, Nucl. Phys. B 573, 617.

Chishtie, F. A., and V. Elias, 2001, Phys. Lett. B 521, 434.

Choi, S. K., et al. (Belle Collaboration), 2003, Phys. Rev. Lett. 91, 262001.

Cinabro, D., et al. (CLEO Collaboration), 2002, e-print hep-ex/ 0207062.

Contreras, C., G. Cvetic, and P. Gaete, 2004, Phys. Rev. D 70, 034008.

Curci, G., A. Di Giacomo, and G. Paffuti, 1983, Z. Phys. C 18, 135.

Cvetic, G., 2004, J. Phys. G 30, 863.

Czarnecki, A., and A. G. Grozin, 1997, Phys. Lett. B 405, 142.

Czarnecki, A., and K. Melnikov, 1998, Phys. Rev. Lett. 80, 2531.

Czarnecki, A., and K. Melnikov, 2001, Phys. Lett. B 519, 212.

Czarnecki, A., and K. Melnikov, 2002, Phys. Rev. D 65, 051501.

Czarnecki, A., K. Melnikov, and A. Yelkhovsky, 1999a, Phys. Rev. Lett. 82, 311.

Czarnecki, A., K. Melnikov, and A. Yelkhovsky, 1999b, Phys. Rev. A 59, 4316.

De Rujula, A., and S. L. Glashow, 1975, Phys. Rev. Lett. 34, 46.

D'Elia, M., A. Di Giacomo, and E. Meggiolaro, 1997, Phys. Lett. B 408, 315.

Di Giacomo, A., H. G. Dosch, V. I. Shevchenko, and Y. A. Simonov, 2002, Phys. Rep. 372, 319.

Dirac, P. A. M., 1949, Rev. Mod. Phys. 21, 392.

Dosch, H. G., and Y. A. Simonov, 1988, Phys. Lett. B 205, 339.

Eichten, E., and F. Feinberg, 1981, Phys. Rev. D 23, 2724.

Eichten, E., K. Gottfried, T. Kinoshita, K. D. Lane, and T.-M. Yan, 1978, Phys. Rev. D 17, 3090.

Eichten, E., and B. Hill, 1990, Phys. Lett. B 243, 427.

Eichten, E. J., and C. Quigg, 1995, Phys. Rev. D 52, 1726.

Eidelman, S., et al. (Particle Data Group), 2004, Phys. Lett. B 592, 1.

Eidemüller, M., 2003, Phys. Rev. D 67, 113002.

Eiras, D., and J. Soto, 2000, Phys. Lett. B 491, 101.

Fadin, V. S., and V. A. Khoze, 1988, Sov. J. Nucl. Phys. 48, 309. 
Falk, A. F., B. Grinstein, and M. E. Luke, 1991, Nucl. Phys. B 357, 185.

Field, J. H., 2002, Phys. Rev. D 66, 013013.

Fischler, W., 1977, Nucl. Phys. B 129, 157.

Fleming, S., and A. K. Leibovich, 2003a, Phys. Rev. D 67, 074035.

Fleming, S., and A. K. Leibovich, 2003b, Phys. Rev. Lett. 90, 032001.

Fleming, S., and A. K. Leibovich, 2004, Phys. Rev. D 70 , 094016.

Fleming, S., I. Z. Rothstein, and A. K. Leibovich, 2001, Phys. Rev. D 64, 036002.

Foldy, L. L., 1961, Phys. Rev. 122, 275.

Foster, M., and C. Michael (UKQCD Collaboration), 1999, Phys. Rev. D 59, 094509.

Fujii, H., and D. Kharzeev, 1999, Phys. Rev. D 60, 114039.

Garcia i Tormo, X., and J. Soto, 2004, Phys. Rev. D 69, 114006.

Gray, A., et al. (HPQCD Collaboration), 2003, Nucl. Phys. B,

Proc. Suppl. 119, 592.

Gray, N., D. J. Broadhurst, W. Grafe, and K. Schilcher, 1990, Z. Phys. C 48, 673.

Gremm, M., and A. Kapustin, 1997, Phys. Lett. B 407, 323.

Griesshammer, H. W., 1998, Phys. Rev. D 58, 094027.

Griesshammer, H. W., 2000, Nucl. Phys. B 579, 313.

Grinstein, B., and I. Z. Rothstein, 1998, Phys. Rev. D 57, 78.

Gromes, D., 1984, Z. Phys. C 26, 401.

Groote, S., and A. A. Pivovarov, 2002, JETP Lett. 75, 221.

Gupta, S. N., and S. F. Radford, 1981, Phys. Rev. D 24, 2309.

Gupta, S. N., and S. F. Radford, 1982, Phys. Rev. D 25, 3430.

Gupta, S. N., W. W. Repko, and I. C. J. Suchyta, 1989, Phys.

Rev. D 40, 4100.

Hagiwara, K., C. B. Kim, and T. Yoshino, 1981, Nucl. Phys. B 177, 461.

Harris, I., and L. Brown, 1957, Phys. Rev. 105, 1656.

Herb, S. W., et al., 1977, Phys. Rev. Lett. 39, 252.

Hill, R., and G. P. Lepage, 2000, Phys. Rev. D 62, 111301.

Hill, R. J., 2001, Phys. Rev. Lett. 86, 3280.

Hill, R. J., and M. Neubert, 2003, Nucl. Phys. B 657, 229.

Hoang, A. H., 1999, Phys. Rev. D 59, 014039.

Hoang, A. H., 2000, e-print hep-ph/0008102.

Hoang, A. H., 2002, e-print hep-ph/0204299.

Hoang, A. H., 2003, Acta Phys. Pol. B 34, 4491.

Hoang, A. H., 2004, Phys. Rev. D 69, 034009.

Hoang, A. H., P. Labelle, and S. M. Zebarjad, 1997, Phys. Rev. Lett. 79, 3387.

Hoang, A. H., Z. Ligeti, and A. V. Manohar, 1999, Phys. Rev. Lett. 82, 277.

Hoang, A. H., A. V. Manohar, and I. W. Stewart, 2001, Phys. Rev. D 64, 014033.

Hoang, A. H., A. V. Manohar, I. W. Stewart, and T. Teubner, 2001, Phys. Rev. Lett. 86, 1951.

Hoang, A. H., A. V. Manohar, I. W. Stewart, and T. Teubner, 2002, Phys. Rev. D 65, 014014.

Hoang, A. H., M. C. Smith, T. Stelzer, and S. Willenbrock, 1999, Phys. Rev. D 59, 114014.

Hoang, A. H., and I. W. Stewart, 2003, Phys. Rev. D 67, 114020 .

Hoang, A. H., and T. Teubner, 1999, Phys. Rev. D 60, 114027.

Hoang, A. H., et al., 2000, Eur. Phys. J. C 2, 3.

Huntley, A., and C. Michael, 1987, Nucl. Phys. B 286, 211.

Isgur, N., and J. Paton, 1985, Phys. Rev. D 31, 2910.

Juge, K. J., J. Kuti, and C. Morningstar, 2003, Phys. Rev. Lett. 90, 161601.
Källen, G., and A. Sarby, 1955, K. Dan. Vidensk. Selsk. Mat. Fys. Medd. 29 N17, 1.

Kinoshita, T., and M. Nio, 1996, Phys. Rev. D 53, 4909.

Kiyo, Y., and Y. Sumino, 2000, Phys. Lett. B 496, 83.

Kniehl, B. A., and A. A. Penin, 1999, Nucl. Phys. B 563, 200.

Kniehl, B. A., and A. A. Penin, 2000a, Phys. Rev. Lett. 85, 1210.

Kniehl, B. A., and A. A. Penin, 2000b, Phys. Rev. Lett. 85, 5094.

Kniehl, B. A., and A. A. Penin, 2000c, Nucl. Phys. B 577, 197. Kniehl, B. A., A. A. Penin, A. Pineda, V. A. Smirnov, and M. Steinhauser, 2004, Phys. Rev. Lett. 92, 242001.

Kniehl, B. A., A. A. Penin, Y. Schroder, V. A. Smirnov, and M. Steinhauser, 2005, Phys. Lett. B 607, 96.

Kniehl, B. A., A. A. Penin, V. A. Smirnov, and M. Steinhauser, 2002a, Nucl. Phys. B 635, 357.

Kniehl, B. A., A. A. Penin, M. Steinhauser, and V. A. Smirnov, 2002b, Phys. Rev. D 65, 091503.

Kniehl, B. A., A. A. Penin, M. Steinhauser, and V. A. Smirnov, 2003, Phys. Rev. Lett. 90, 212001.

Koller, K., and T. Walsh, 1978, Nucl. Phys. B 140, 449.

Krajcik, R. A., and L. L. Foldy, 1974, Phys. Rev. D 10, 1777.

Krämer, A., H. G. Dosch, and R. A. Bertlmann, 1992, Fortschr. Phys. 40, 93.

Krämer, M., 1999, Phys. Rev. D 60, 111503.

Krämer, M., 2001, Prog. Part. Nucl. Phys. 47, 141.

Kronfeld, A. S., 1998, Phys. Rev. D 58, 051501.

Kronfeld, A. S., 2004, Nucl. Phys. B, Proc. Suppl. 129, 46.

Kuhn, J. H., A. A. Penin, and A. A. Pivovarov, 1998, Nucl. Phys. B 534, 356.

Kummer, W., W. Mödritsch, and A. Vairo, 1996, Z. Phys. C 72, 653.

Labelle, P., 1998, Phys. Rev. D 58, 093013.

Labelle, P., S. M. Zebarjad, and C. P. Burgess, 1997, Phys. Rev. D 56, 8053 .

Le Guillou, J. C., and J. Zinn-Justin, 1990, Eds., Large Order Behavior of Perturbation Theory (Current Physics-Sources and Comments) (North-Holland, Amsterdam).

Lee, T., 2003a, J. High Energy Phys. 10, 044.

Lee, T., 2003b, Phys. Rev. D 67, 014020.

Lepage, G. P., 1997, e-print nucl-th/9706029.

Lepage, G. P., 2005, Ann. Phys. (N.Y.) 315, 193.

Lepage, G. P., and B. A. Thacker, 1988, Nucl. Phys. B, Proc. Suppl. 4, 199.

Lepage, G. P., et al., 1992, Phys. Rev. D 46, 4052.

Lepage, P., and C. Davies, 2004, Int. J. Mod. Phys. A 19, 877. Leutwyler, H., 1981, Phys. Lett. 98B, 447.

Lucha, W., F. F. Schöberl, and D. Gromes, 1991, Phys. Rep. 200, 127.

Lucini, B., and M. Teper, 2001, J. High Energy Phys. 06, 050. Luke, M. E., and A. V. Manohar, 1992, Phys. Lett. B 286, 348. Luke, M. E., and A. V. Manohar, 1997, Phys. Rev. D 55, 4129. Luke, M. E., A. V. Manohar, and I. Z. Rothstein, 2000, Phys. Rev. D 61, 074025.

Luke, M. E., and M. J. Savage, 1998, Phys. Rev. D 57, 413.

Lüscher, M., and P. Weisz, 2002, J. High Energy Phys. 07, 049.

Lüscher, M., and P. Weisz, 2004, J. High Energy Phys. 07, 014.

Ma, J. P., and Q. Wang, 2002, Phys. Lett. B 537, 233.

Mackenzie, P. B., and G. P. Lepage, 1981, Phys. Rev. Lett. 47, 1244.

Maltoni, F., 1999, Ph.D. thesis (University of Pisa).

Maltoni, F., 2000, e-print hep-ph/0007003.

Maltoni, F., and A. Petrelli, 1999, Phys. Rev. D 59, 074006. 
Mannel, T., and R. Urech, 1997, Z. Phys. C 73, 541.

Manohar, A. V., 1997, Phys. Rev. D 56, 230.

Manohar, A. V., and P. Ruiz-Femenia, 2004, Phys. Rev. D 69, 053003.

Manohar, A. V., and I. W. Stewart, 2000a, Phys. Rev. Lett. 85, 2248.

Manohar, A. V., and I. W. Stewart, 2000b, Phys. Rev. D 62, 074015.

Manohar, A. V., and I. W. Stewart, 2000c, Phys. Rev. D 62, 014033.

Manohar, A. V., and I. W. Stewart, 2001, Phys. Rev. D 63, 054004.

Martinelli, G., et al., 1997, Phys. Lett. B 411, 141.

Martinez, M., and R. Miquel, 2003, Eur. Phys. J. C 27, 49.

Matsui, T., and H. Satz, 1986, Phys. Lett. B 178, 416.

Mattson, M., et al. (SELEX Collaboration), 2002, Phys. Rev. Lett. 89, 112001.

Melles, M., 2000, Phys. Rev. D 62, 074019.

Melnikov, K., and T. v. Ritbergen, 2000, Phys. Lett. B 482, 99.

Melnikov, K., and A. Yelkhovsky, 1998, Nucl. Phys. B 528, 59.

Melnikov, K., and A. Yelkhovsky, 1999a, Phys. Rev. D 59, 114009.

Melnikov, K., and A. Yelkhovsky, 1999b, Phys. Lett. B 458, 143.

Melnikov, K., and A. Yelkhovsky, 2000, Phys. Rev. D 62, 116003.

Melnikov, K., and A. Yelkhovsky, 2001, Phys. Rev. Lett. 86, 1498.

Messiah, A., 1979, Quantum Mechanics (De Gruyter, Berlin), Vol. 2.

Michael, C., 1986, Phys. Rev. Lett. 56, 1219.

Migdal, A. A., 1983, Phys. Rep. 102, 199.

Morningstar, C. J., and M. J. Peardon, 1999, Phys. Rev. D 60, 034509.

Necco, S., and R. Sommer, 2001, Phys. Lett. B 523, 135.

Necco, S., and R. Sommer, 2002, Nucl. Phys. B 622, 328.

Nemati, B., et al. (CLEO Collaboration), 1997, Phys. Rev. D 55, 5273.

Neubert, M., 1994, Phys. Rep. 245, 259.

Neubert, M., and C. T. Sachrajda, 1995, Nucl. Phys. B 438, 235.

Onishchenko, A. I., 2000, e-print hep-ph/0005127.

Pantaleone, J., S. H. H. Tye, and Y. J. Ng, 1986, Phys. Rev. D 33, 777.

Pascual, P., and R. Tarrach, 1984, Lect. Notes Phys. 194, 1.

Penin, A. A., A. Pineda, V. A. Smirnov, and M. Steinhauser, 2004a, Phys. Lett. B 593, 124.

Penin, A. A., A. Pineda, V. A. Smirnov, and M. Steinhauser, 2004b, Nucl. Phys. B 699, 183.

Penin, A. A., and A. A. Pivovarov, 1998, Phys. Lett. B 435, 413.

Penin, A. A., and A. A. Pivovarov, 1999, Nucl. Phys. B 549, 217.

Penin, A. A., and A. A. Pivovarov, 2001, Phys. At. Nucl. 64, 275.

Penin, A. A., and M. Steinhauser, 2002, Phys. Lett. B 538, 335. Peskin, M. E., 1979, Nucl. Phys. B 156, 365.

Peskin, M. E., 1983, presented at 11th International SLAC Summer Institute on Particle Physics, Stanford, CA, July 1826.

Peter, M., 1997, Phys. Rev. Lett. 78, 602.

Petrelli, A., M. Cacciari, M. Greco, F. Maltoni, and M. L. Mangano, 1998, Nucl. Phys. B 514, 245.

Photiadis, D. M., 1985, Phys. Lett. 164B, 160.
Pineda, A., 1997a, Phys. Rev. D 55, 407.

Pineda, A., 1997b, Nucl. Phys. B 494, 213.

Pineda, A., 1998, Ph.D. thesis (University of Barcelona).

Pineda, A., 2001, J. High Energy Phys. 06, 022.

Pineda, A., 2002a, Phys. Rev. D 66, 054022.

Pineda, A., 2002b, Phys. Rev. D 65, 074007.

Pineda, A., 2002c, Phys. Rev. A 66, 062108.

Pineda, A., 2003a, Acta Phys. Pol. B 34, 5295.

Pineda, A., 2003b, J. Phys. G 29, 371.

Pineda, A., 2004, Nucl. Phys. B, Proc. Suppl. 133, 190.

Pineda, A., and J. Soto, 1998a, Nucl. Phys. B, Proc. Suppl. 64, 428.

Pineda, A., and J. Soto, 1998b, Phys. Lett. B 420, 391.

Pineda, A., and J. Soto, 1998c, Phys. Rev. D 58, 114011.

Pineda, A., and J. Soto, 1999, Phys. Rev. D 59, 016005.

Pineda, A., and J. Soto, 2000, Phys. Lett. B 495, 323.

Pineda, A., and A. Vairo, 2001, Phys. Rev. D 63, 054007.

Pineda, A., and F. J. Yndurain, 1998, Phys. Rev. D 58, 094022.

Pineda, A., and F. J. Yndurain, 2000, Phys. Rev. D 61, 077505.

Portoles, J., and P. D. Ruiz-Femenia, 2002, Eur. Phys. J. C 24, 439.

Recksiegel, S., and Y. Sumino, 2002, Phys. Rev. D 65, 054018. Recksiegel, S., and Y. Sumino, 2003, Eur. Phys. J. C 31, 187.

Recksiegel, S., and Y. Sumino, 2004, Phys. Lett. B 578, 369.

Rösch, T., 2003, Diploma thesis (University of Heidelberg).

Rothstein, I. Z., and M. B. Wise, 1997, Phys. Lett. B 402, 346.

Scherer, S., and H. W. Fearing, 1995, Phys. Rev. D 52, 6445.

Schröder, Y., 1999a, Ph.D. thesis (University of Hamburg), DESY-THESIS-1999-021.

Schröder, Y., 1999b, Phys. Lett. B 447, 321.

Sebastian, K. J., and D. Yun, 1979, Phys. Rev. D 19, 2509.

Skwarnicki, T., 2004, Int. J. Mod. Phys. A 19, 1030.

Sommer, R., 1994, Nucl. Phys. B 411, 839.

Soto, J., 2003, e-print hep-ph/0301138.

Strassler, M. J., and M. E. Peskin, 1991, Phys. Rev. D 43, 1500.

Sumino, Y., 2002, Phys. Rev. D 65, 054003.

Susskind, L., 1977, in Les Houches 1976, Proceedings, Weak and Electromagnetic Interactions at High Energies (NorthHolland, Amsterdam), pp. 207-308.

Szczepaniak, A. P., and E. S. Swanson, 1997, Phys. Rev. D 55, 3987.

Thacker, B. A., and G. P. Lepage, 1991, Phys. Rev. D 43, 196. Titard, S., and F. J. Yndurain, 1994, Phys. Rev. D 49, 6007.

Titard, S., and F. J. Yndurain, 1995, Phys. Rev. D 51, 6348.

Trottier, H. D., and G. P. Lepage, 1998, Nucl. Phys. B, Proc. Suppl. 63, 865 .

Vairo, A., 2000, e-print hep-ph/0010191.

Vairo, A., 2002, e-print hep-ph/0212271.

Vairo, A., 2003, Nucl. Phys. B, Proc. Suppl. 115, 166.

Vairo, A., 2004a, Nucl. Phys. B, Proc. Suppl. 133, 196.

Vairo, A., 2004b, Mod. Phys. Lett. A 19, 253.

Voloshin, M. B., 1979, Nucl. Phys. B 154, 365.

Voloshin, M. B., 1982, Sov. J. Nucl. Phys. 36, 143.

Voloshin, M. B., 1995, Int. J. Mod. Phys. A 10, 2865.

Voloshin, M. B., 2003, Phys. Lett. B 562, 68.

Voloshin, M. B., 2004, Mod. Phys. Lett. A 19, 181.

Wang, H.-b., and Y.-P. Yao, 2004, Phys. Rev. D 70, 094046.

Wilson, K. G., 1974, Phys. Rev. D 10, 2445.

Wolf, S., 2001, Phys. Rev. D 63, 074020.

Yakovlev, O. I., and S. Groote, 2001, Phys. Rev. D 63, 074012.

Yelkhovsky, A., 2001, Phys. Rev. A 64, 062104.

Yndurain, F. J., 1999, The Theory of Quark and Gluon Interactions (Springer, Berlin). 